\title{
Partial Ionization Cross Sections of Organic Molecules
}

\author{
Karl K. Irikura \\ National Institute of Standards and Technology, Gaithersburg, MD 20899, USA \\ karl.irikura@nist.gov \\ Partial ionization cross sections are the absolute yields of specific ions from an electron-molecule collision. They are necessary for \\ modeling plasmas and for determining the sensitivity of mass spectrometers, among other applications. One mass-spectrometric \\ application is estimating the abundance of organic compounds on Mars, as sampled by the rover Curiosity. This is a report of \\ semitheoretical data obtained for a collection of organic molecules identified as possible biomarkers in this exotic context.
}

Key words: cross section; electron ionization; gas analysis; mass spectrometry; quantum chemistry.

Accepted: May 11, 2017

Published: June 13, 2017

https://doi.org/10.6028/jres.122.028

\section{Introduction}

One of the experiments aboard the National Aeronautics and Space Administration's (NASA's) Martian rover Curiosity is a mass-spectrometric search for organic compounds, as possible markers of life. In this measurement, a solid sample is heated, and the evolved gases are sampled using a quadrupole mass spectrometer (QMS), which constitutes part of the remote laboratory called SAM (Sample Analysis at Mars) [1]. When an organic compound is detected, an approximate estimate of its abundance is desired. Absolute QMS ion intensities may be used to infer this abundance.

The quantitation method may be illustrated using simple equations. Ideally, the absolute intensity, $I_{\mathrm{A}}$, of a peak in a mass spectrum will be proportional to the number density $\left(p_{\mathrm{A}}\right)$ of the gaseous analyte,

$$
p_{\mathrm{A}}=I_{\mathrm{A}} / C \text {. }
$$

The proportionality constant, $C$, is normally determined by calibration, using a series of known pressures of the analyte. However, this cannot be done remotely on Mars, and it is not done before launch because it is not known which molecules will be detected. To proceed anyway, note that $C$ includes intrinsic and extrinsic effects: the partial ionization cross section (i.e., intrinsic ion yield) [2], $\sigma_{\mathrm{A}}$, and the responsivity of the instrument, $\beta$. This is shown in Eq. (2). Partial ionization cross sections have units of area.

$$
p_{\mathrm{A}}=I_{\mathrm{A}} /\left(\sigma_{\mathrm{A}} \beta\right) \text {. }
$$

To use carbon dioxide as an internal calibrant, we combine Eq. (2) for two gases, $A$ and $\mathrm{CO}_{2}$, to obtain

$$
p_{\mathrm{A}}=\frac{I_{\mathrm{A}}}{I_{\mathrm{CO} 2}} \frac{\sigma_{\mathrm{CO} 2}}{\sigma_{\mathrm{A}}} p_{\mathrm{CO2}} \text {. }
$$

Note that it has been assumed, as an approximation, that the instrument responsivity is the same for the ion representing $A$ as for the ion representing $\mathrm{CO}_{2}$. The QMS provides in situ values for the corresponding peak intensities $I_{\mathrm{A}}$ and $I_{\mathrm{CO} 2}$. The instrument was calibrated for $\mathrm{CO}_{2}$ on Earth, before launch. If we believe the calibration has not drifted far, then we know $p_{\mathrm{CO} 2}$ from $I_{\mathrm{CO} 2}$ and Eq. (1). Partial ionization cross sections are known for $\mathrm{CO}_{2}$ [3]. Thus, the only missing datum in Eq. (3) is $\sigma_{\mathrm{A}}$, the partial ionization cross section (PICS) for the peak due to the analyte. Estimating this PICS is the focus of this report. 


\section{Method}

\subsection{Summary of theory}

PICS values, which are intrinsic molecular properties, are estimated by combining an $a b$ initio total ionization cross section (TICS) with empirical ion-branching fractions, as described in detail elsewhere [4] and summarized below. All ions are assumed to be singly positive, so that the symbol $m$ (mass) is used where $\mathrm{m} / \mathrm{z}$ (mass-to-charge ratio) might be expected.

The TICS is the sum of all PICS for a given analyte and is computed here from first principles using the binary-encounter Bethe (BEB) theory developed by Yong-Ki Kim and coworkers [5-13]. However, there are no purely theoretical methods for predicting the relative intensities of fragment ions. Instead, we use standard electron-ionization mass spectra (EIMS) from the National Institute of Standards and Technology (NIST) Chemistry WebBook [14]. For each ion, the relative intensity from the experimental spectrum is denoted $I_{\text {EIMS }}(m)$, where $m$ is the ion mass. The relative intensities in ordinary EIMS reflect the relative PICS values but suffer systematic bias against low-mass fragment ions [15-20]. A correction, $w(m)$, is applied to mitigate systematic, mass-dependent measurement bias, as shown in Eq. (4) and Eq. (5). The resulting, corrected, normalized branching fractions are denoted $I_{\text {corr }}(m) . M$ is the molecular mass, and $b$ is a parameter that was obtained by fitting benchmark PICS measurements, here taken as $b=0.87$.

$$
\begin{gathered}
I_{\text {corr }}(m)=\frac{w(m) I_{\mathrm{EIMS}}(m)}{\sum_{m} w(m) I_{\mathrm{EIMS}}(m)} . \\
1(m)=\left\{\begin{array}{cc}
1 & \text { for } m \geq b M \\
\sqrt{b /(1-b)} \sqrt{(M-m) / m} & \text { for } m<b M
\end{array} .\right.
\end{gathered}
$$

The details and rationale for this correction are described elsewhere [4]. Briefly, the correction is based upon a rudimentary physical model that attributes mass discrimination to high kinetic-energy release during the formation of fragment ions. Low-mass ions (with a heavy coproduct) have greater velocity than highmass ions (with a light coproduct) because of conservation of momentum. Fast ions are more likely to follow "bad" trajectories in a mass spectrometer and fail to be detected. Such mass discrimination is unimportant in analytical chemistry because the low-mass ions are seldom chemically distinctive.

The BEB calculation requires binding energies and kinetic energies for the molecular orbitals in the target molecule. These are obtained from quantum chemistry calculations. In the present work, molecular geometry optimization was done using the popular, hybrid density functional B3LYP [21, 22] using 6$31 \mathrm{G}(\mathrm{d})$ basis sets. Binding and kinetic energies for all orbitals were computed at the Hartree-Fock (HF) level using 6-311G(d,p) basis sets. For thiophenol, orbital kinetic energies for outer orbitals were recalculated using the "SDDall" effective core potential (ECP) and basis set on the sulfur center [23]. Binding energies for outer orbitals were recalculated (all-electron) using electron propagator theory [24], which includes electron correlation. Finally, the molecular thresholds for vertical single and double ionization were computed using the heavily correlated CCSD(T) theory (coupled-cluster with single and double excitations and a perturbative estimate of triples) [25] along with cc-pV(T+d)Z basis sets [26]. Inhouse, Python-language software was used to create the quantum chemistry input files and to parse the resulting output files. The quantum chemistry calculations were done using the Gaussian09 software [27]. ${ }^{1}$

\subsection{Experimental orbital binding energies}

When experimental vertical binding energies $(B)$ are available from published photoelectron spectroscopy (PES), they may be used instead of theoretical values. The PES values used here, along with the $a b$ initio values that they replace, are listed below for all affected molecules.

${ }^{1}$ Certain commercial materials and equipment are identified in this paper in order to specify procedures completely. In no case does such identification imply recommendation or endorsement by the National Institute of Standards and Technology, nor does it imply that the material or equipment identified is necessarily the best available for the purpose. 
Thiophenol: For the five highest-lying orbitals, values of $\mathrm{B} / \mathrm{eV}=8.49,9.41,10.61,11.61$, and 12.22 [28] replaced theoretical, $a b$ initio values of $B / e V=8.39$ [CCSD(T)], 9.42 (P3 propagator theory), 10.47 (P3), 11.69 (P3), and 12.40 (P3).

$o$-Xylene and $m$-xylene: For the highest-lying molecular orbital, $B=8.56 \mathrm{eV}$ [29] replaced the theoretical CCSD(T) value of $8.70 \mathrm{eV}$.

p-Xylene: For the HOMO, $B=8.44 \mathrm{eV}$ [29] replaced the theoretical CCSD(T) value of $8.58 \mathrm{eV}$.

Styrene: For three highest orbitals, values of $B / \mathrm{eV}=8.49,9.27$, and 10.55 [30] replaced the

corresponding theoretical values of 8.56 [CCSD(T)], 9.22 (P3), and $10.71(\mathrm{P} 3)$.

1-Hexene, 2-hexene, and 3-hexene: For the HOMOs, $B / \mathrm{eV}=9.65,9.16$, and 9.14 [31] replaced the corresponding $\operatorname{CCSD}(\mathrm{T})$ values of $9.71,9.21$, and 9.20 for these three compounds, respectively.

2,3-Dimethyl-2-butene: For the highest orbitals, $B / \mathrm{eV}=8.44,10.96$, and 12.71 [32] replaced the theoretical values of 8.60 [CCSD(T)], 11.09 (P3), and $12.30(\mathrm{P} 3)$, respectively.

1-Heptene, 2-heptene, and 3-heptene: For the HOMOs, $B / \mathrm{eV}=9.68,9.11$, and 9.05 [33] replaced the CCSD(T) values of 9.69, 9.18, and 9.14, respectively.

Benzaldehyde: The HOMO binding energy of $9.80 \mathrm{eV}$ [34] replaced the CCSD(T) value of $9.71 \mathrm{eV}$.

Phenol: For the highest orbitals, $B / \mathrm{eV}=8.70,9.39,11.59,12.02,12.61$, and 13.44 [28] replaced the theoretical CCSD(T) value of 8.58 and the P3 values of 9.37, 11.73, 12.19, 12.76, and 13.72, respectively.

Acetophenone: For the highest orbitals, $B / \mathrm{eV}=9.37,9.55,9.77$, and 11.91 [35] replaced the CCSD(T) value of 9.38 and the $\mathrm{P} 3$ values of $9.59,9.55$, and 12.21 , respectively.

Pyrrole: For the two highest orbitals, $B / \mathrm{eV}=8.209$ and 9.20 [36] replaced the $\operatorname{CCSD}(\mathrm{T})$ value of 8.26 and the $\mathrm{P} 3$ value of 9.05 , respectively.

Pyridine: For the highest orbitals, $B / \mathrm{eV}=9.60,9.75,10.51$, and 12.61 [28] replaced the theoretical CCSD(T) value of 9.69 and the P3 values of 9.81, 10.42, and 12.93, respectively.

Benzonitrile: For the highest orbitals, $B / \mathrm{eV}=9.71,10.17,11.84$, and 12.09 [28] replaced the theoretical CCSD(T) value of 9.80 and the P3 values of 10.04, 12.29, and 12.46, respectively.

Experimental EIMS data were taken from the NIST WebBook [14] when available. These are generally "consensus" spectra that combine numerous individual measurements [37]. For 3-methyl-2hexene, the mass spectrum was not specifically for the $E$ isomer. For 2-methyl-3-hexene, the EIMS was not in the WebBook and was taken directly from the NIST mass spectral database [38]. For 2-ethyl-1-pentene, no EIMS data are available in the NIST database or in the literature, so PICS could not be computed.

\section{Results}

\subsection{Total ionization cross sections (TICS)}

The list of molecules of interest was selected for relevance to the search for organic compounds on Mars [34]. Some of these molecules exist in multiple isomeric forms. All such isomers were considered here, except for isomers with cis double bonds, which were omitted because they are expected to be less stable than their trans counterparts. See Table 1 for the complete list of isomers studied. Also included in Table 1 are the BEB values of the TICS. Their associated standard uncertainty is about 13\% [13]. In Table 1 , some values were computed purely theoretically, and some were computed using available binding energies from experimental PES. In the latter situations, the differences from purely theoretical values are all less than $1 \%$.

Molecular-orbital binding energies and kinetic energies are summarized in the data tables, one for each molecule studied. The tables are presented in the Appendix, below, and are also available, in the supplemental materials, as tab-delimited text files named " $<$ molecule $>$.bun." The suffix "bun" derives from the symbols in the BEB equations for orbital binding energy $(B)$, kinetic energy $(U)$, and occupation number $(N)$. Also available in the supplemental materials is a Perl-language script, "beb_tbl.pl," which reads a BUN file, applies the BEB theory, and produces a table of TICS as a function of incident electron energy. It is very similar to the script behind the NIST database [35]. For the present application, the only interesting value of the incident electron energy is $70 \mathrm{eV}\left(1 \mathrm{eV} \approx 1.602 \times 10^{-19} \mathrm{~J}\right)$. This is also the energy for which EIMS data are most abundant. However, the BUN files and Perl script may be used to compute 
TICS at any value of the incident electron energy. For very high energies, the relativistic version of BEB [10], which is not implemented in the Perl script, would be more appropriate.

Table 1. Molecular names, structures, and TICS at $70 \mathrm{eV}$.

\begin{tabular}{|c|c|c|c|c|c|}
\hline Name & Structure & TICS $/ 10^{-20} \mathrm{~m}^{2}$ & Name & Structure & TICS $/ 10^{-20} \mathrm{~m}^{2}$ \\
\hline thiophenol & & 19.06 & 3-methyl-2-hexene & & 21.27 \\
\hline$o$-xylene & & 21.52 & 4-methyl-2-hexene & & 21.40 \\
\hline$m$-xylene & & 21.76 & 5-methyl-2-hexene & & 21.31 \\
\hline p-xylene & & 21.76 & 2-methyl-3-hexene & & 21.25 \\
\hline styrene & & 20.60 & 3-methyl-3-hexene & & 21.47 \\
\hline 1-hexene & & 18.04 & 2,3-dimethyl-1-pentene & & 21.43 \\
\hline 2-hexene & & 17.98 & 2,4-dimethyl-1-pentene & & 21.59 \\
\hline 3-hexene & & 18.42 & 3,4-dimethyl-1-pentene & & 21.40 \\
\hline 2-methyl-1-pentene & & 17.74 & 3,3-dimethyl-1-pentene & & 21.14 \\
\hline 3-methyl-1-pentene & & 18.15 & 4,4-dimethyl-1-pentene & & 20.65 \\
\hline 4-methyl-1-pentene & & 17.96 & 2,3-dimethyl-2-pentene & & 21.77 \\
\hline 2-methyl-2-pentene & & 18.30 & 2,4-dimethyl-2-pentene & & 20.71 \\
\hline 3-methyl-2-pentene & & 18.15 & 3,4-dimethyl-2-pentene & & 20.57 \\
\hline 4-methyl-2-pentene & & 18.10 & 4,4-dimethyl-2-pentene & & 21.59 \\
\hline 2,3-dimethyl-1-butene & & 17.84 & 2-ethyl-1-pentene & & 21.08 \\
\hline 3,3-dimethyl-1-butene & & 17.67 & 3-ethyl-1-pentene & & 21.09 \\
\hline 2,3-dimethyl-2-butene & & 18.57 & 3-ethyl-2-pentene & & 21.49 \\
\hline 2-ethyl-1-butene & & 18.17 & 2,3,3-trimethyl-1-butene & & 20.97 \\
\hline 1-heptene & & 21.03 & 2-ethyl-3-methyl-1-butene & & 21.23 \\
\hline
\end{tabular}




2-methyl-2-hexene

\subsection{Partial ionization cross sections (PICS)}

The estimated PICS at $70 \mathrm{eV}$ are tabulated below, starting with Table 2 . They are also provided in the supplemental materials, in computer-readable form. There is no table for 2-ethyl-1-pentene because the necessary EIMS data are unavailable. In addition to the PICS value for each ion, $67 \%$ and $95 \%$ uncertainty intervals are provided. Note that these intervals are not symmetric about the recommended value. The $67 \%$ uncertainty intervals are probably reliable [4]. For the strongest peak in each mass spectrum (the "base peak"), the 95\% uncertainty intervals are probably also reliable, but for other peaks, the $95 \%$ intervals may be too narrow [4]. The last column in each PICS table is the uncertainty model used in the Monte Carlo determination of the uncertainty intervals: model " $\mathrm{A}$ " is linear in intensity, " $\mathrm{B}$ " is logarithmic, and " $\mathrm{H}$ " is a crude estimate for $\mathrm{H}^{+}(\mathrm{m} / \mathrm{z} 1)$. Details of the uncertainty models are provided elsewhere [4]. A cursory look at the tables reveals that the uncertainties are much larger than would be obtained by laboratory calibration. Fortunately, the values are sufficiently precise for the Mars application, which is semiquantitative. 
Table 2. Partial ionization cross sections $\left(10^{-20} \mathrm{~m}^{2}\right)$ for thiophenol. TICS $/ 10^{-20} \mathrm{~m}^{2}=19.060 . M=110$ is base peak.

\begin{tabular}{|c|c|c|c|c|c|c|c|c|c|}
\hline$m / z$ & PICS & $67 \%$ interval & $95 \%$ interval & unc. model & $m / z$ & PICS & $67 \%$ interval & $95 \%$ interval & unc. model \\
\hline 1 & 0.400 & {$[0.288,0.478]$} & {$[0.215,0.600]$} & $\mathrm{H}$ & 65 & 0.888 & {$[0.458,1.455]$} & {$[0.227,2.343]$} & B \\
\hline 17 & 0.023 & {$[0.011,0.036]$} & {$[0.006,0.063]$} & B & 66 & 2.527 & {$[0.724,3.357]$} & {$[0.137,5.134]$} & A \\
\hline 18 & 0.158 & {$[0.078,0.250]$} & {$[0.040,0.422]$} & B & 67 & 0.135 & {$[0.066,0.213]$} & {$[0.034,0.361]$} & B \\
\hline 26 & 0.072 & {$[0.035,0.112]$} & {$[0.018,0.193]$} & B & 68 & 0.024 & {$[0.011,0.037]$} & {$[0.006,0.063]$} & B \\
\hline 27 & 0.191 & {$[0.094,0.300]$} & {$[0.049,0.512]$} & B & 69 & 0.538 & {$[0.272,0.869]$} & {$[0.138,1.434]$} & B \\
\hline 28 & 0.186 & {$[0.092,0.295]$} & {$[0.047,0.497]$} & B & 70 & 0.060 & {$[0.029,0.094]$} & {$[0.015,0.162]$} & B \\
\hline 32 & 0.062 & {$[0.030,0.097]$} & {$[0.016,0.167]$} & B & 71 & 0.133 & {$[0.065,0.209]$} & {$[0.034,0.354]$} & B \\
\hline 33 & 0.046 & {$[0.022,0.071]$} & {$[0.012,0.122]$} & B & 72 & 0.007 & {$[0.003,0.011]$} & {$[0.002,0.019]$} & B \\
\hline 34 & 0.015 & {$[0.007,0.023]$} & {$[0.004,0.040]$} & B & 73 & 0.043 & {$[0.021,0.067]$} & {$[0.011,0.114]$} & B \\
\hline 36 & 0.029 & {$[0.014,0.045]$} & {$[0.007,0.077]$} & B & 74 & 0.139 & {$[0.068,0.218]$} & {$[0.035,0.370]$} & B \\
\hline 37 & 0.209 & {$[0.103,0.331]$} & {$[0.053,0.559]$} & B & 75 & 0.081 & {$[0.040,0.127]$} & {$[0.021,0.217]$} & B \\
\hline 38 & 0.301 & {$[0.149,0.478]$} & {$[0.076,0.797]$} & B & 76 & 0.073 & {$[0.036,0.115]$} & {$[0.019,0.194]$} & B \\
\hline 39 & 1.171 & {$[0.613,1.942]$} & {$[0.301,3.086]$} & B & 77 & 0.804 & {$[0.411,1.309]$} & {$[0.206,2.128]$} & B \\
\hline 40 & 0.079 & {$[0.039,0.124]$} & {$[0.020,0.212]$} & B & 78 & 0.159 & {$[0.078,0.251]$} & {$[0.040,0.422]$} & B \\
\hline 44 & 0.086 & {$[0.042,0.135]$} & {$[0.022,0.228]$} & B & 79 & 0.006 & {$[0.003,0.010]$} & {$[0.002,0.017]$} & B \\
\hline 45 & 0.695 & {$[0.353,1.126]$} & {$[0.177,1.835]$} & B & 80 & 0.006 & {$[0.003,0.009]$} & {$[0.002,0.016]$} & B \\
\hline 46 & 0.012 & {$[0.006,0.018]$} & {$[0.003,0.032]$} & B & 81 & 0.077 & {$[0.038,0.121]$} & {$[0.020,0.207]$} & B \\
\hline 47 & 0.035 & {$[0.017,0.054]$} & {$[0.009,0.093]$} & B & 82 & 0.128 & {$[0.063,0.203]$} & {$[0.033,0.342]$} & B \\
\hline 48 & 0.011 & {$[0.005,0.018]$} & {$[0.003,0.030]$} & B & 83 & 0.062 & {$[0.030,0.098]$} & {$[0.016,0.167]$} & B \\
\hline 49 & 0.067 & {$[0.033,0.105]$} & {$[0.017,0.178]$} & B & 84 & 0.755 & {$[0.386,1.230]$} & {$[0.193,1.997]$} & B \\
\hline 50 & 0.590 & {$[0.299,0.954]$} & {$[0.150,1.561]$} & B & 85 & 0.038 & {$[0.018,0.059]$} & {$[0.010,0.102]$} & B \\
\hline 51 & 1.020 & {$[0.528,1.673]$} & {$[0.260,2.677]$} & B & 86 & 0.032 & {$[0.015,0.049]$} & {$[0.008,0.085]$} & B \\
\hline 52 & 0.095 & {$[0.047,0.149]$} & {$[0.024,0.253]$} & B & 92 & 0.004 & {$[0.002,0.007]$} & {$[0.001,0.012]$} & B \\
\hline 53 & 0.041 & {$[0.020,0.065]$} & {$[0.011,0.111]$} & B & 93 & 0.017 & {$[0.008,0.027]$} & {$[0.004,0.046]$} & B \\
\hline 54 & 0.131 & {$[0.064,0.206]$} & {$[0.033,0.350]$} & B & 95 & 0.032 & {$[0.015,0.049]$} & {$[0.008,0.085]$} & B \\
\hline 55 & 0.209 & {$[0.103,0.330]$} & {$[0.053,0.555]$} & B & 105 & 0.008 & {$[0.004,0.012]$} & {$[0.002,0.021]$} & B \\
\hline 56 & 0.020 & {$[0.010,0.031]$} & {$[0.005,0.053]$} & B & 106 & 0.008 & {$[0.004,0.012]$} & {$[0.002,0.021]$} & B \\
\hline 57 & 0.144 & {$[0.071,0.226]$} & {$[0.037,0.382]$} & B & 107 & 0.008 & {$[0.004,0.012]$} & {$[0.002,0.021]$} & B \\
\hline 58 & 0.254 & {$[0.126,0.403]$} & {$[0.065,0.678]$} & B & 108 & 0.096 & {$[0.047,0.151]$} & {$[0.024,0.255]$} & B \\
\hline 59 & 0.065 & {$[0.032,0.102]$} & {$[0.017,0.173]$} & B & 109 & 0.930 & {$[0.479,1.522]$} & {$[0.238,2.463]$} & B \\
\hline 60 & 0.027 & {$[0.013,0.042]$} & {$[0.007,0.073]$} & B & 110 & 3.861 & {$[2.189,5.066]$} & {$[1.123,6.759]$} & A \\
\hline 61 & 0.081 & {$[0.039,0.126]$} & {$[0.021,0.215]$} & B & 111 & 0.324 & {$[0.162,0.517]$} & {$[0.082,0.859]$} & B \\
\hline 62 & 0.105 & {$[0.051,0.165]$} & {$[0.027,0.279]$} & B & 112 & 0.185 & {$[0.091,0.292]$} & {$[0.047,0.492]$} & B \\
\hline 63 & 0.198 & {$[0.097,0.312]$} & {$[0.051,0.529]$} & B & 113 & 0.012 & {$[0.006,0.018]$} & {$[0.003,0.031]$} & B \\
\hline 64 & 0.034 & {$[0.016,0.053]$} & {$[0.009,0.091]$} & B & & & & & \\
\hline
\end{tabular}


Table 3. Partial ionization cross sections $\left(10^{-20} \mathrm{~m}^{2}\right)$ for $o$-xylene. TICS $/ 10^{-20} \mathrm{~m}^{2}=21.518 . M=106$, base peak is $\mathrm{m} / \mathrm{z} 91$.

\begin{tabular}{|c|c|c|c|c|c|c|c|c|c|}
\hline$m / z$ & PICS & $67 \%$ interval & $95 \%$ interval & unc. model & $m / z$ & PICS & $67 \%$ interval & $95 \%$ interval & unc. model \\
\hline 1 & 0.400 & {$[0.287,0.477]$} & {$[0.216,0.600]$} & $\mathrm{H}$ & 74 & 0.164 & {$[0.080,0.258]$} & {$[0.042,0.436]$} & B \\
\hline 15 & 0.097 & {$[0.048,0.153]$} & {$[0.025,0.258]$} & B & 75 & 0.101 & {$[0.049,0.158]$} & {$[0.026,0.271]$} & B \\
\hline 26 & 0.092 & {$[0.045,0.145]$} & {$[0.024,0.247]$} & B & 76 & 0.099 & {$[0.048,0.156]$} & {$[0.025,0.267]$} & B \\
\hline 27 & 0.494 & {$[0.247,0.791]$} & {$[0.126,1.309]$} & B & 77 & 1.071 & {$[0.558,1.776]$} & {$[0.274,2.832]$} & B \\
\hline 29 & 0.021 & {$[0.010,0.033]$} & {$[0.005,0.058]$} & B & 78 & 0.574 & {$[0.289,0.925]$} & {$[0.146,1.522]$} & B \\
\hline 37 & 0.090 & {$[0.044,0.141]$} & {$[0.023,0.240]$} & B & 79 & 0.575 & {$[0.290,0.925]$} & {$[0.147,1.526]$} & B \\
\hline 38 & 0.246 & {$[0.122,0.391]$} & {$[0.063,0.656]$} & B & 80 & 0.037 & {$[0.018,0.059]$} & {$[0.010,0.101]$} & B \\
\hline 39 & 1.342 & {$[0.709,2.242]$} & {$[0.344,3.528]$} & B & 85 & 0.013 & {$[0.006,0.020]$} & {$[0.003,0.035]$} & B \\
\hline 40 & 0.135 & {$[0.067,0.214]$} & {$[0.034,0.362]$} & B & 86 & 0.025 & {$[0.012,0.040]$} & {$[0.007,0.068]$} & B \\
\hline 41 & 0.182 & {$[0.090,0.289]$} & {$[0.047,0.488]$} & B & 87 & 0.025 & {$[0.012,0.039]$} & {$[0.006,0.066]$} & B \\
\hline 43 & 0.016 & {$[0.008,0.025]$} & {$[0.004,0.043]$} & B & 88 & 0.006 & {$[0.003,0.009]$} & {$[0.002,0.016]$} & B \\
\hline 45 & 0.015 & {$[0.007,0.024]$} & {$[0.004,0.041]$} & B & 89 & 0.149 & {$[0.074,0.237]$} & {$[0.038,0.400]$} & B \\
\hline 49 & 0.071 & {$[0.035,0.112]$} & {$[0.018,0.191]$} & B & 90 & 0.006 & {$[0.003,0.009]$} & {$[0.001,0.015]$} & B \\
\hline 50 & 0.708 & {$[0.359,1.146]$} & {$[0.180,1.871]$} & B & 91 & 5.327 & {$[3.409,6.599]$} & {$[2.135,8.486]$} & A \\
\hline 51 & 1.499 & {$[0.796,2.503]$} & {$[0.387,3.946]$} & B & 92 & 0.415 & {$[0.208,0.666]$} & {$[0.106,1.105]$} & B \\
\hline 52 & 0.602 & {$[0.302,0.965]$} & {$[0.153,1.593]$} & B & 93 & 0.015 & {$[0.007,0.024]$} & {$[0.004,0.041]$} & B \\
\hline 53 & 0.341 & {$[0.171,0.548]$} & {$[0.087,0.909]$} & B & 97 & 0.005 & {$[0.002,0.008]$} & {$[0.001,0.014]$} & B \\
\hline 54 & 0.026 & {$[0.012,0.040]$} & {$[0.007,0.069]$} & B & 98 & 0.015 & {$[0.007,0.024]$} & {$[0.004,0.041]$} & B \\
\hline 55 & 0.013 & {$[0.006,0.020]$} & {$[0.003,0.034]$} & B & 99 & 0.005 & {$[0.002,0.008]$} & {$[0.001,0.014]$} & B \\
\hline 60 & 0.011 & {$[0.006,0.018]$} & {$[0.003,0.031]$} & B & 101 & 0.010 & {$[0.005,0.016]$} & {$[0.003,0.027]$} & B \\
\hline 61 & 0.101 & {$[0.050,0.161]$} & {$[0.026,0.273]$} & B & 102 & 0.076 & {$[0.037,0.119]$} & {$[0.019,0.204]$} & B \\
\hline 62 & 0.265 & {$[0.132,0.421]$} & {$[0.068,0.708]$} & B & 103 & 0.345 & {$[0.172,0.549]$} & {$[0.088,0.917]$} & B \\
\hline 63 & 0.683 & {$[0.346,1.104]$} & {$[0.175,1.811]$} & B & 104 & 0.127 & {$[0.063,0.201]$} & {$[0.032,0.339]$} & B \\
\hline 64 & 0.117 & {$[0.057,0.185]$} & {$[0.030,0.315]$} & B & 105 & 1.045 & {$[0.542,1.718]$} & {$[0.267,2.747]$} & B \\
\hline 65 & 0.813 & {$[0.414,1.319]$} & {$[0.207,2.155]$} & B & 106 & 2.546 & {$[1.459,4.362]$} & {$[0.658,6.353]$} & B \\
\hline 66 & 0.092 & {$[0.045,0.145]$} & {$[0.024,0.246]$} & B & 107 & 0.223 & {$[0.110,0.352]$} & {$[0.057,0.596]$} & B \\
\hline 67 & 0.010 & {$[0.005,0.016]$} & {$[0.003,0.027]$} & B & 108 & 0.010 & {$[0.005,0.016]$} & {$[0.003,0.027]$} & B \\
\hline 73 & 0.026 & {$[0.013,0.041]$} & {$[0.007,0.071]$} & B & & & & & \\
\hline
\end{tabular}

Table 4. Partial ionization cross sections $\left(10^{-20} \mathrm{~m}^{2}\right)$ for $m$-xylene. TICS $/ 10^{-20} \mathrm{~m}^{2}=21.758 . M=106$, base peak is $\mathrm{m} / \mathrm{z} 91$.

\begin{tabular}{|c|c|c|c|c|c|c|c|c|c|}
\hline$m / z$ & PICS & $67 \%$ interval & $95 \%$ interval & unc. model & $m / z$ & PICS & $67 \%$ interval & $95 \%$ interval & unc. mode \\
\hline 1 & 0.400 & {$[0.288,0.478]$} & {$[0.215,0.599]$} & $\mathrm{H}$ & 74 & 0.153 & {$[0.075,0.241]$} & {$[0.039,0.407]$} & B \\
\hline 15 & 0.096 & {$[0.047,0.150]$} & {$[0.024,0.255]$} & B & 75 & 0.100 & {$[0.049,0.157]$} & {$[0.025,0.266]$} & B \\
\hline 26 & 0.091 & {$[0.044,0.142]$} & {$[0.023,0.243]$} & B & 76 & 0.089 & {$[0.043,0.140]$} & {$[0.023,0.239]$} & B \\
\hline 27 & 0.487 & {$[0.243,0.776]$} & {$[0.124,1.288]$} & B & 77 & 1.071 & {$[0.554,1.759]$} & {$[0.274,2.824]$} & B \\
\hline 29 & 0.021 & {$[0.010,0.033]$} & {$[0.005,0.056]$} & B & 78 & 0.581 & {$[0.293,0.936]$} & {$[0.148,1.538]$} & B \\
\hline 37 & 0.106 & {$[0.052,0.166]$} & {$[0.027,0.284]$} & B & 79 & 0.559 & {$[0.281,0.898]$} & {$[0.143,1.483]$} & B \\
\hline 38 & 0.277 & {$[0.137,0.439]$} & {$[0.070,0.735]$} & B & 80 & 0.037 & {$[0.018,0.057]$} & {$[0.009,0.098]$} & B \\
\hline 39 & 1.475 & {$[0.779,2.457]$} & {$[0.377,3.851]$} & B & 85 & 0.013 & {$[0.006,0.020]$} & {$[0.003,0.035]$} & B \\
\hline 40 & 0.166 & {$[0.082,0.262]$} & {$[0.042,0.443]$} & B & 86 & 0.031 & {$[0.015,0.049]$} & {$[0.008,0.083]$} & B \\
\hline 41 & 0.179 & {$[0.088,0.283]$} & {$[0.046,0.479]$} & B & 87 & 0.030 & {$[0.015,0.047]$} & {$[0.008,0.081]$} & B \\
\hline 43 & 0.016 & {$[0.008,0.024]$} & {$[0.004,0.042]$} & B & 88 & 0.006 & {$[0.003,0.009]$} & {$[0.001,0.016]$} & B \\
\hline 45 & 0.015 & {$[0.007,0.023]$} & {$[0.004,0.040]$} & B & 89 & 0.153 & {$[0.075,0.239]$} & {$[0.039,0.407]$} & B \\
\hline 49 & 0.056 & {$[0.027,0.087]$} & {$[0.014,0.149]$} & B & 90 & 0.049 & {$[0.024,0.077]$} & {$[0.012,0.131]$} & B \\
\hline 50 & 0.630 & {$[0.317,1.011]$} & {$[0.161,1.674]$} & B & 91 & 5.250 & {$[3.318,6.703]$} & {$[2.011,8.716]$} & A \\
\hline 51 & 1.424 & {$[0.748,2.364]$} & {$[0.365,3.727]$} & B & 92 & 0.404 & {$[0.202,0.645]$} & {$[0.103,1.074]$} & B \\
\hline 52 & 0.633 & {$[0.317,1.013]$} & {$[0.162,1.686]$} & B & 93 & 0.015 & {$[0.007,0.023]$} & {$[0.004,0.040]$} & B \\
\hline 53 & 0.349 & {$[0.174,0.556]$} & {$[0.089,0.928]$} & B & 97 & 0.005 & {$[0.002,0.008]$} & {$[0.001,0.013]$} & B \\
\hline 54 & 0.025 & {$[0.012,0.040]$} & {$[0.006,0.068]$} & B & 98 & 0.015 & {$[0.007,0.023]$} & {$[0.004,0.040]$} & B \\
\hline 55 & 0.012 & {$[0.006,0.019]$} & {$[0.003,0.033]$} & B & 99 & 0.005 & {$[0.002,0.008]$} & {$[0.001,0.013]$} & B \\
\hline 60 & 0.011 & {$[0.005,0.018]$} & {$[0.003,0.031]$} & B & 101 & 0.010 & {$[0.005,0.015]$} & {$[0.003,0.027]$} & B \\
\hline 61 & 0.111 & {$[0.054,0.175]$} & {$[0.028,0.297]$} & B & 102 & 0.070 & {$[0.034,0.110]$} & {$[0.018,0.186]$} & B \\
\hline 62 & 0.294 & {$[0.146,0.466]$} & {$[0.075,0.786]$} & B & 103 & 0.340 & {$[0.169,0.542]$} & {$[0.087,0.904]$} & B \\
\hline 63 & 0.727 & {$[0.368,1.174]$} & {$[0.185,1.915]$} & B & 104 & 0.115 & {$[0.056,0.180]$} & {$[0.029,0.307]$} & B \\
\hline 64 & 0.136 & {$[0.067,0.215]$} & {$[0.035,0.363]$} & B & 105 & 1.160 & {$[0.602,1.908]$} & {$[0.297,3.049]$} & B \\
\hline 65 & 0.781 & {$[0.396,1.261]$} & {$[0.200,2.065]$} & B & 106 & 2.604 & {$[0.473,3.357]$} & {$[0.040,5.526]$} & A \\
\hline 66 & 0.101 & {$[0.049,0.158]$} & {$[0.026,0.270]$} & B & 107 & 0.230 & {$[0.114,0.364]$} & {$[0.059,0.615]$} & B \\
\hline 67 & 0.010 & {$[0.005,0.015]$} & {$[0.003,0.027]$} & B & 108 & 0.010 & {$[0.005,0.015]$} & {$[0.003,0.027]$} & B \\
\hline 73 & 0.026 & {$[0.013,0.041]$} & {$[0.007,0.070]$} & B & & & & & \\
\hline
\end{tabular}


Table 5. Partial ionization cross sections $\left(10^{-20} \mathrm{~m}^{2}\right)$ for $p$-xylene. TICS $/ 10^{-20} \mathrm{~m}^{2}=21.758 . M=106$, base peak is $\mathrm{m} / z 91$.

\begin{tabular}{|c|c|c|c|c|c|c|c|c|c|}
\hline$m / z$ & PICS & $67 \%$ interval & $95 \%$ interval & unc. model & $m / z$ & PICS & $67 \%$ interval & $95 \%$ interval & unc. model \\
\hline 1 & 0.400 & {$[0.289,0.479]$} & {$[0.215,0.600]$} & $\mathrm{H}$ & 69 & 0.010 & {$[0.005,0.015]$} & {$[0.002,0.026]$} & B \\
\hline 15 & 0.033 & {$[0.016,0.051]$} & {$[0.008,0.088]$} & B & 73 & 0.018 & {$[0.009,0.028]$} & {$[0.005,0.048]$} & B \\
\hline 18 & 0.059 & {$[0.029,0.092]$} & {$[0.015,0.157]$} & B & 74 & 0.112 & {$[0.055,0.177]$} & {$[0.029,0.303]$} & B \\
\hline 26 & 0.070 & {$[0.034,0.109]$} & {$[0.018,0.185]$} & B & 75 & 0.068 & {$[0.033,0.107]$} & {$[0.017,0.183]$} & B \\
\hline 27 & 0.790 & {$[0.403,1.285]$} & {$[0.201,2.087]$} & B & 76 & 0.066 & {$[0.032,0.104]$} & {$[0.017,0.179]$} & B \\
\hline 28 & 0.177 & {$[0.087,0.280]$} & {$[0.046,0.475]$} & B & 77 & 0.965 & {$[0.495,1.578]$} & {$[0.245,2.541]$} & B \\
\hline 29 & 0.022 & {$[0.010,0.034]$} & {$[0.006,0.058]$} & B & 78 & 0.459 & {$[0.230,0.735]$} & {$[0.117,1.217]$} & B \\
\hline 32 & 0.040 & {$[0.020,0.063]$} & {$[0.010,0.109]$} & B & 79 & 0.548 & {$[0.277,0.884]$} & {$[0.141,1.463]$} & B \\
\hline 37 & 0.072 & {$[0.035,0.113]$} & {$[0.019,0.194]$} & B & 80 & 0.030 & {$[0.015,0.047]$} & {$[0.008,0.081]$} & B \\
\hline 38 & 0.211 & {$[0.104,0.335]$} & {$[0.054,0.566]$} & B & 83 & 0.007 & {$[0.003,0.011]$} & {$[0.002,0.019]$} & B \\
\hline 39 & 1.386 & {$[0.735,2.327]$} & {$[0.355,3.642]$} & B & 85 & 0.007 & {$[0.003,0.010]$} & {$[0.002,0.018]$} & B \\
\hline 40 & 0.119 & {$[0.059,0.189]$} & {$[0.031,0.320]$} & B & 86 & 0.026 & {$[0.012,0.040]$} & {$[0.007,0.068]$} & B \\
\hline 41 & 0.215 & {$[0.107,0.344]$} & {$[0.055,0.581]$} & B & 87 & 0.025 & {$[0.012,0.039]$} & {$[0.006,0.066]$} & B \\
\hline 43 & 0.016 & {$[0.008,0.025]$} & {$[0.004,0.043]$} & B & 89 & 0.109 & {$[0.054,0.173]$} & {$[0.028,0.294]$} & B \\
\hline 49 & 0.057 & {$[0.028,0.091]$} & {$[0.015,0.155]$} & B & 90 & 0.039 & {$[0.019,0.061]$} & {$[0.010,0.105]$} & B \\
\hline 50 & 0.559 & {$[0.281,0.898]$} & {$[0.142,1.479]$} & B & 91 & 5.371 & {$[3.444,6.750]$} & {$[2.156,8.732]$} & A \\
\hline 51 & 1.180 & {$[0.613,1.945]$} & {$[0.302,3.109]$} & B & 92 & 0.387 & {$[0.192,0.615]$} & {$[0.099,1.034]$} & B \\
\hline 52 & 0.457 & {$[0.229,0.733]$} & {$[0.117,1.218]$} & B & 93 & 0.010 & {$[0.005,0.016]$} & {$[0.003,0.028]$} & B \\
\hline 53 & 0.369 & {$[0.183,0.586]$} & {$[0.094,0.977]$} & B & 97 & 0.005 & {$[0.002,0.008]$} & {$[0.001,0.014]$} & B \\
\hline 54 & 0.013 & {$[0.006,0.020]$} & {$[0.003,0.035]$} & B & 98 & 0.005 & {$[0.002,0.008]$} & {$[0.001,0.014]$} & B \\
\hline 55 & 0.025 & {$[0.012,0.040]$} & {$[0.007,0.069]$} & B & 101 & 0.010 & {$[0.005,0.016]$} & {$[0.003,0.028]$} & B \\
\hline 57 & 0.025 & {$[0.012,0.038]$} & {$[0.006,0.066]$} & B & 102 & 0.056 & {$[0.027,0.087]$} & {$[0.014,0.150]$} & B \\
\hline 61 & 0.080 & {$[0.039,0.126]$} & {$[0.020,0.215]$} & B & 103 & 0.301 & {$[0.149,0.477]$} & {$[0.077,0.803]$} & B \\
\hline 62 & 0.177 & {$[0.088,0.281]$} & {$[0.046,0.477]$} & B & 104 & 0.148 & {$[0.073,0.236]$} & {$[0.038,0.396]$} & B \\
\hline 63 & 0.513 & {$[0.257,0.821]$} & {$[0.130,1.352]$} & B & 105 & 1.477 & {$[0.785,2.479]$} & {$[0.379,3.889]$} & B \\
\hline 64 & 0.096 & {$[0.047,0.153]$} & {$[0.025,0.262]$} & B & 106 & 3.370 & {$[1.411,4.425]$} & {$[0.508,6.370]$} & A \\
\hline 65 & 0.587 & {$[0.297,0.946]$} & {$[0.152,1.568]$} & B & 107 & 0.296 & {$[0.148,0.473]$} & {$[0.076,0.794]$} & B \\
\hline 66 & 0.072 & {$[0.035,0.113]$} & {$[0.019,0.194]$} & B & 108 & 0.005 & {$[0.002,0.008]$} & {$[0.001,0.014]$} & B \\
\hline 67 & 0.010 & {$[0.005,0.016]$} & {$[0.003,0.027]$} & B & & & & & \\
\hline
\end{tabular}

Table 6. Partial ionization cross sections $\left(10^{-20} \mathrm{~m}^{2}\right)$ for styrene. TICS $/ 10^{-20} \mathrm{~m}^{2}=20.601 . M=104$ is base peak.

\begin{tabular}{|c|c|c|c|c|c|c|c|c|c|}
\hline$m / z$ & PICS & $67 \%$ interval & $95 \%$ interval & unc. model & $m / z$ & PICS & $67 \%$ interval & $95 \%$ interval & unc. model \\
\hline 1 & 0.400 & {$[0.288,0.478]$} & {$[0.215,0.599]$} & $\mathrm{H}$ & 72 & 0.007 & {$[0.003,0.010]$} & {$[0.002,0.018]$} & B \\
\hline 15 & 0.024 & {$[0.012,0.037]$} & {$[0.006,0.065]$} & B & 73 & 0.077 & {$[0.038,0.122]$} & {$[0.020,0.208]$} & B \\
\hline 25 & 0.018 & {$[0.009,0.028]$} & {$[0.005,0.048]$} & B & 74 & 0.397 & {$[0.199,0.635]$} & {$[0.102,1.057]$} & B \\
\hline 26 & 0.154 & {$[0.076,0.244]$} & {$[0.040,0.414]$} & B & 75 & 0.265 & {$[0.133,0.426]$} & {$[0.068,0.707]$} & B \\
\hline 27 & 0.351 & {$[0.175,0.560]$} & {$[0.090,0.938]$} & B & 76 & 0.312 & {$[0.155,0.495]$} & {$[0.080,0.831]$} & B \\
\hline 36 & 0.014 & {$[0.007,0.021]$} & {$[0.003,0.036]$} & B & 77 & 1.284 & {$[0.667,2.112]$} & {$[0.327,3.359]$} & B \\
\hline 37 & 0.147 & {$[0.072,0.230]$} & {$[0.037,0.388]$} & B & 78 & 2.652 & {$[0.676,3.463]$} & {$[0.143,5.550]$} & A \\
\hline 38 & 0.261 & {$[0.130,0.416]$} & {$[0.066,0.692]$} & B & 79 & 0.173 & {$[0.085,0.273]$} & {$[0.044,0.462]$} & B \\
\hline 39 & 0.869 & {$[0.443,1.409]$} & {$[0.220,2.276]$} & B & 80 & 0.005 & {$[0.003,0.008]$} & {$[0.001,0.015]$} & B \\
\hline 40 & 0.038 & {$[0.018,0.059]$} & {$[0.010,0.101]$} & B & 84 & 0.005 & {$[0.002,0.008]$} & {$[0.001,0.013]$} & B \\
\hline 41 & 0.025 & {$[0.012,0.039]$} & {$[0.006,0.067]$} & B & 85 & 0.014 & {$[0.007,0.022]$} & {$[0.004,0.037]$} & B \\
\hline 43 & 0.012 & {$[0.006,0.018]$} & {$[0.003,0.032]$} & B & 86 & 0.023 & {$[0.011,0.035]$} & {$[0.006,0.061]$} & B \\
\hline 48 & 0.011 & {$[0.005,0.017]$} & {$[0.003,0.029]$} & B & 87 & 0.035 & {$[0.017,0.055]$} & {$[0.009,0.094]$} & B \\
\hline 49 & 0.147 & {$[0.072,0.230]$} & {$[0.038,0.394]$} & B & 88 & 0.004 & {$[0.002,0.007]$} & {$[0.001,0.011]$} & B \\
\hline 50 & 1.399 & {$[0.729,2.305]$} & {$[0.358,3.667]$} & B & 89 & 0.085 & {$[0.042,0.136]$} & {$[0.022,0.230]$} & B \\
\hline 51 & 2.695 & {$[0.739,3.544]$} & {$[0.156,5.599]$} & A & 90 & 0.008 & {$[0.004,0.012]$} & {$[0.002,0.021]$} & B \\
\hline 52 & 0.871 & {$[0.447,1.419]$} & {$[0.224,2.309]$} & B & 97 & 0.008 & {$[0.004,0.012]$} & {$[0.002,0.021]$} & B \\
\hline 53 & 0.117 & {$[0.057,0.185]$} & {$[0.030,0.313]$} & B & 98 & 0.034 & {$[0.017,0.054]$} & {$[0.009,0.093]$} & B \\
\hline 54 & 0.010 & {$[0.005,0.015]$} & {$[0.002,0.026]$} & B & 99 & 0.008 & {$[0.004,0.012]$} & {$[0.002,0.021]$} & B \\
\hline 60 & 0.017 & {$[0.008,0.026]$} & {$[0.004,0.046]$} & B & 101 & 0.034 & {$[0.017,0.054]$} & {$[0.009,0.093]$} & B \\
\hline 61 & 0.133 & {$[0.065,0.209]$} & {$[0.034,0.355]$} & B & 102 & 0.321 & {$[0.160,0.512]$} & {$[0.082,0.859]$} & B \\
\hline 62 & 0.269 & {$[0.133,0.428]$} & {$[0.069,0.718]$} & B & 103 & 1.856 & {$[0.998,3.128]$} & {$[0.473,4.806]$} & B \\
\hline 63 & 0.631 & {$[0.318,1.015]$} & {$[0.162,1.675]$} & B & 104 & 3.827 & {$[1.959,5.000]$} & {$[0.965,6.961]$} & A \\
\hline 64 & 0.070 & {$[0.035,0.111]$} & {$[0.018,0.189]$} & B & 105 & 0.352 & {$[0.175,0.561]$} & {$[0.090,0.943]$} & B \\
\hline 65 & 0.115 & {$[0.057,0.182]$} & {$[0.030,0.309]$} & B & 106 & 0.011 & {$[0.006,0.018]$} & {$[0.003,0.031]$} & B \\
\hline 66 & 0.008 & {$[0.004,0.012]$} & {$[0.002,0.020]$} & B & & & & & \\
\hline
\end{tabular}


Table 7. Partial ionization cross sections $\left(10^{-20} \mathrm{~m}^{2}\right)$ for 1-hexene. TICS $/ 10^{-20} \mathrm{~m}^{2}=18.038 . M=84$, base peak is $\mathrm{m} / \mathrm{z} 56$.

\begin{tabular}{|c|c|c|c|c|c|c|c|c|c|}
\hline$m / z$ & PICS & $67 \%$ interval & $95 \%$ interval & unc. model & $m / z$ & PICS & $67 \%$ interval & $95 \%$ interval & unc. model \\
\hline 1 & 0.400 & {$[0.288,0.478]$} & {$[0.214,0.598]$} & $\mathrm{H}$ & 56 & 2.395 & {$[0.672,3.116]$} & {$[0.171,4.955]$} & A \\
\hline 15 & 0.065 & {$[0.032,0.103]$} & {$[0.017,0.176]$} & B & 57 & 0.142 & {$[0.070,0.225]$} & {$[0.037,0.382]$} & B \\
\hline 26 & 0.106 & {$[0.052,0.167]$} & {$[0.027,0.283]$} & B & 58 & 0.002 & {$[0.001,0.004]$} & {$[0.001,0.006]$} & B \\
\hline 27 & 1.584 & {$[0.849,2.663]$} & {$[0.405,4.119]$} & B & 61 & 0.002 & {$[0.001,0.003]$} & {$[0.001,0.006]$} & B \\
\hline 28 & 0.277 & {$[0.138,0.440]$} & {$[0.071,0.738]$} & B & 62 & 0.006 & {$[0.003,0.009]$} & {$[0.002,0.016]$} & B \\
\hline 29 & 0.867 & {$[0.442,1.408]$} & {$[0.220,2.280]$} & B & 63 & 0.010 & {$[0.005,0.015]$} & {$[0.003,0.027]$} & B \\
\hline 30 & 0.018 & {$[0.009,0.028]$} & {$[0.005,0.049]$} & B & 65 & 0.015 & {$[0.007,0.023]$} & {$[0.004,0.039]$} & B \\
\hline 37 & 0.031 & {$[0.015,0.048]$} & {$[0.008,0.083]$} & B & 66 & 0.004 & {$[0.002,0.005]$} & {$[0.001,0.010]$} & B \\
\hline 38 & 0.089 & {$[0.044,0.141]$} & {$[0.023,0.239]$} & B & 67 & 0.036 & {$[0.017,0.056]$} & {$[0.009,0.096]$} & B \\
\hline 39 & 1.124 & {$[0.581,1.839]$} & {$[0.287,2.934]$} & B & 68 & 0.007 & {$[0.003,0.010]$} & {$[0.002,0.018]$} & B \\
\hline 40 & 0.216 & {$[0.108,0.344]$} & {$[0.055,0.578]$} & B & 69 & 0.379 & {$[0.190,0.607]$} & {$[0.097,1.007]$} & B \\
\hline 41 & 3.299 & {$[1.648,4.283]$} & {$[0.810,6.027]$} & A & 70 & 0.021 & {$[0.010,0.033]$} & {$[0.005,0.057]$} & B \\
\hline 42 & 2.445 & {$[0.739,3.189]$} & {$[0.196,5.003]$} & A & 71 & 0.001 & {$[0.001,0.002]$} & {$[0.000,0.004]$} & B \\
\hline 43 & 1.945 & {$[1.066,3.304]$} & {$[0.500,5.010]$} & B & 74 & 0.003 & {$[0.001,0.004]$} & {$[0.001,0.007]$} & B \\
\hline 44 & 0.064 & {$[0.031,0.100]$} & {$[0.016,0.173]$} & B & 77 & 0.005 & {$[0.003,0.008]$} & {$[0.001,0.014]$} & B \\
\hline 49 & 0.006 & {$[0.003,0.009]$} & {$[0.001,0.016]$} & B & 78 & 0.001 & {$[0.001,0.002]$} & {$[0.000,0.004]$} & B \\
\hline 50 & 0.056 & {$[0.027,0.088]$} & {$[0.014,0.150]$} & B & 79 & 0.004 & {$[0.002,0.006]$} & {$[0.001,0.011]$} & B \\
\hline 51 & 0.079 & {$[0.038,0.124]$} & {$[0.020,0.212]$} & B & 81 & 0.003 & {$[0.001,0.004]$} & {$[0.001,0.007]$} & B \\
\hline 52 & 0.032 & {$[0.015,0.049]$} & {$[0.008,0.085]$} & B & 83 & 0.004 & {$[0.002,0.006]$} & {$[0.001,0.011]$} & B \\
\hline 53 & 0.176 & {$[0.087,0.278]$} & {$[0.045,0.469]$} & B & 84 & 0.382 & {$[0.190,0.609]$} & {$[0.097,1.015]$} & B \\
\hline 54 & 0.131 & {$[0.064,0.206]$} & {$[0.034,0.352]$} & B & 85 & 0.026 & {$[0.013,0.041]$} & {$[0.007,0.070]$} & B \\
\hline 55 & 1.579 & {$[0.842,2.645]$} & {$[0.403,4.099]$} & B & 86 & 0.003 & {$[0.001,0.004]$} & {$[0.001,0.007]$} & B \\
\hline
\end{tabular}

Table 8. Partial ionization cross sections $\left(10^{-20} \mathrm{~m}^{2}\right)$ for 2-hexene. TICS $/ 10^{-20} \mathrm{~m}^{2}=17.975 . M=84$, base peak is $m / z 55$.

\begin{tabular}{|c|c|c|c|c|c|c|c|c|c|}
\hline$m / z$ & PICS & $67 \%$ interval & $95 \%$ interval & unc. model & $m / z$ & PICS & $67 \%$ interval & $95 \%$ interval & unc. model \\
\hline 1 & 0.400 & {$[0.288,0.478]$} & {$[0.215,0.599]$} & $\mathrm{H}$ & 54 & 0.262 & {$[0.130,0.415]$} & {$[0.067,0.699]$} & B \\
\hline 14 & 0.034 & {$[0.017,0.054]$} & {$[0.009,0.092]$} & B & 55 & 3.698 & {$[2.062,4.741]$} & {$[1.149,6.470]$} & A \\
\hline 15 & 0.119 & {$[0.059,0.188]$} & {$[0.031,0.320]$} & B & 56 & 0.972 & {$[0.499,1.586]$} & {$[0.246,2.543]$} & B \\
\hline 26 & 0.144 & {$[0.071,0.228]$} & {$[0.037,0.386]$} & B & 57 & 0.056 & {$[0.027,0.087]$} & {$[0.014,0.150]$} & B \\
\hline 27 & 1.487 & {$[0.791,2.494]$} & {$[0.379,3.884]$} & B & 61 & 0.003 & {$[0.002,0.005]$} & {$[0.001,0.008]$} & B \\
\hline 28 & 0.151 & {$[0.074,0.237]$} & {$[0.038,0.403]$} & B & 62 & 0.009 & {$[0.004,0.014]$} & {$[0.002,0.024]$} & B \\
\hline 29 & 1.549 & {$[0.833,2.611]$} & {$[0.399,4.054]$} & B & 63 & 0.012 & {$[0.006,0.018]$} & {$[0.003,0.032]$} & B \\
\hline 30 & 0.034 & {$[0.017,0.053]$} & {$[0.009,0.091]$} & B & 65 & 0.022 & {$[0.011,0.035]$} & {$[0.006,0.060]$} & B \\
\hline 37 & 0.034 & {$[0.017,0.054]$} & {$[0.009,0.093]$} & B & 66 & 0.008 & {$[0.004,0.012]$} & {$[0.002,0.022]$} & B \\
\hline 38 & 0.095 & {$[0.046,0.149]$} & {$[0.024,0.255]$} & B & 67 & 0.074 & {$[0.036,0.116]$} & {$[0.019,0.200]$} & B \\
\hline 39 & 1.214 & {$[0.638,2.011]$} & {$[0.312,3.180]$} & B & 68 & 0.017 & {$[0.008,0.027]$} & {$[0.004,0.047]$} & B \\
\hline 40 & 0.208 & {$[0.103,0.328]$} & {$[0.053,0.552]$} & B & 69 & 0.536 & {$[0.270,0.861]$} & {$[0.137,1.417]$} & B \\
\hline 41 & 2.185 & {$[0.450,2.815]$} & {$[0.067,4.675]$} & A & 70 & 0.027 & {$[0.013,0.043]$} & {$[0.007,0.074]$} & B \\
\hline 42 & 2.566 & {$[0.874,3.371]$} & {$[0.267,5.141]$} & A & 74 & 0.004 & {$[0.002,0.006]$} & {$[0.001,0.011]$} & B \\
\hline 43 & 0.676 & {$[0.343,1.090]$} & {$[0.172,1.778]$} & B & 77 & 0.008 & {$[0.004,0.012]$} & {$[0.002,0.021]$} & B \\
\hline 44 & 0.019 & {$[0.009,0.030]$} & {$[0.005,0.052]$} & B & 78 & 0.002 & {$[0.001,0.003]$} & {$[0.001,0.005]$} & B \\
\hline 45 & 0.009 & {$[0.005,0.015]$} & {$[0.002,0.026]$} & B & 79 & 0.006 & {$[0.003,0.009]$} & {$[0.002,0.016]$} & B \\
\hline 49 & 0.009 & {$[0.004,0.013]$} & {$[0.002,0.023]$} & B & 81 & 0.004 & {$[0.002,0.006]$} & {$[0.001,0.011]$} & B \\
\hline 50 & 0.075 & {$[0.037,0.119]$} & {$[0.019,0.202]$} & B & 83 & 0.008 & {$[0.004,0.012]$} & {$[0.002,0.021]$} & B \\
\hline 51 & 0.110 & {$[0.054,0.174]$} & {$[0.028,0.297]$} & B & 84 & 0.744 & {$[0.378,1.201]$} & {$[0.191,1.972]$} & B \\
\hline 52 & 0.044 & {$[0.021,0.068]$} & {$[0.011,0.118]$} & B & 85 & 0.049 & {$[0.024,0.077]$} & {$[0.013,0.132]$} & B \\
\hline 53 & 0.292 & {$[0.146,0.467]$} & {$[0.075,0.782]$} & B & 86 & 0.002 & {$[0.001,0.003]$} & {$[0.001,0.005]$} & B \\
\hline
\end{tabular}


Table 9. Partial ionization cross sections $\left(10^{-20} \mathrm{~m}^{2}\right)$ for 3-hexene. TICS $/ 10^{-20} \mathrm{~m}^{2}=18.416 . M=84$, base peak is $\mathrm{m} / \mathrm{z} 55$.

\begin{tabular}{|c|c|c|c|c|c|c|c|c|c|}
\hline$m / z$ & PICS & $67 \%$ interval & $95 \%$ interval & unc. model & $m / z$ & PICS & $67 \%$ interval & $95 \%$ interval & unc. model \\
\hline 1 & 0.400 & {$[0.288,0.478]$} & {$[0.216,0.600]$} & $\mathrm{H}$ & 61 & 0.005 & {$[0.002,0.007]$} & {$[0.001,0.013]$} & B \\
\hline 26 & 0.114 & {$[0.056,0.180]$} & {$[0.029,0.304]$} & B & 62 & 0.014 & {$[0.007,0.022]$} & {$[0.004,0.038]$} & B \\
\hline 27 & 1.154 & {$[0.602,1.904]$} & {$[0.293,3.005]$} & B & 63 & 0.031 & {$[0.015,0.048]$} & {$[0.008,0.082]$} & B \\
\hline 28 & 0.158 & {$[0.078,0.249]$} & {$[0.040,0.420]$} & B & 64 & 0.003 & {$[0.002,0.005]$} & {$[0.001,0.009]$} & B \\
\hline 29 & 0.903 & {$[0.465,1.478]$} & {$[0.232,2.385]$} & B & 65 & 0.044 & {$[0.021,0.068]$} & {$[0.011,0.118]$} & B \\
\hline 30 & 0.018 & {$[0.009,0.028]$} & {$[0.005,0.048]$} & B & 66 & 0.019 & {$[0.009,0.030]$} & {$[0.005,0.051]$} & B \\
\hline 37 & 0.045 & {$[0.022,0.070]$} & {$[0.012,0.121]$} & B & 67 & 0.115 & {$[0.056,0.180]$} & {$[0.029,0.308]$} & B \\
\hline 38 & 0.125 & {$[0.062,0.198]$} & {$[0.032,0.336]$} & B & 68 & 0.025 & {$[0.012,0.039]$} & {$[0.006,0.067]$} & B \\
\hline 39 & 1.416 & {$[0.746,2.350]$} & {$[0.362,3.702]$} & B & 69 & 0.610 & {$[0.308,0.985]$} & {$[0.155,1.610]$} & B \\
\hline 40 & 0.229 & {$[0.114,0.365]$} & {$[0.058,0.611]$} & B & 70 & 0.034 & {$[0.017,0.053]$} & {$[0.009,0.092]$} & B \\
\hline 41 & 3.539 & {$[1.864,4.582]$} & {$[0.973,6.348]$} & A & 71 & 0.007 & {$[0.003,0.010]$} & {$[0.002,0.018]$} & B \\
\hline 42 & 2.850 & {$[1.145,3.749]$} & {$[0.419,5.519]$} & A & 74 & 0.007 & {$[0.004,0.011]$} & {$[0.002,0.020]$} & B \\
\hline 43 & 0.597 & {$[0.301,0.961]$} & {$[0.151,1.578]$} & B & 75 & 0.005 & {$[0.003,0.008]$} & {$[0.001,0.014]$} & B \\
\hline 44 & 0.021 & {$[0.010,0.033]$} & {$[0.005,0.057]$} & B & 76 & 0.006 & {$[0.003,0.009]$} & {$[0.001,0.015]$} & B \\
\hline 49 & 0.012 & {$[0.006,0.019]$} & {$[0.003,0.033]$} & B & 77 & 0.017 & {$[0.008,0.027]$} & {$[0.004,0.046]$} & B \\
\hline 50 & 0.106 & {$[0.052,0.167]$} & {$[0.027,0.283]$} & B & 78 & 0.006 & {$[0.003,0.009]$} & {$[0.002,0.016]$} & B \\
\hline 51 & 0.156 & {$[0.077,0.246]$} & {$[0.040,0.417]$} & B & 79 & 0.010 & {$[0.005,0.015]$} & {$[0.002,0.026]$} & B \\
\hline 52 & 0.059 & {$[0.029,0.093]$} & {$[0.015,0.159]$} & B & 81 & 0.008 & {$[0.004,0.012]$} & {$[0.002,0.021]$} & B \\
\hline 53 & 0.287 & {$[0.143,0.456]$} & {$[0.074,0.769]$} & B & 82 & 0.003 & {$[0.002,0.005]$} & {$[0.001,0.009]$} & B \\
\hline 54 & 0.171 & {$[0.085,0.271]$} & {$[0.044,0.459]$} & B & 83 & 0.010 & {$[0.005,0.015]$} & {$[0.002,0.026]$} & B \\
\hline 55 & 3.444 & {$[1.772,4.467]$} & {$[0.893,6.222]$} & A & 84 & 0.729 & {$[0.371,1.181]$} & {$[0.187,1.928]$} & B \\
\hline 56 & 0.803 & {$[0.410,1.304]$} & {$[0.206,2.129]$} & B & 85 & 0.050 & {$[0.025,0.079]$} & {$[0.013,0.135]$} & B \\
\hline 57 & 0.051 & {$[0.025,0.080]$} & {$[0.013,0.136]$} & B & & & & & \\
\hline
\end{tabular}

Table 10. Partial ionization cross sections $\left(10^{-20} \mathrm{~m}^{2}\right)$ for 2-methyl-1-pentene. TICS $/ 10^{-20} \mathrm{~m}^{2}=17.743 . M=84$, base peak is $m / z 56$.

\begin{tabular}{|c|c|c|c|c|c|c|c|c|c|}
\hline$m / z$ & PICS & $67 \%$ interval & $95 \%$ interval & unc. model & $m / \mathbf{z}$ & PICS & $67 \%$ interval & $95 \%$ interval & unc. model \\
\hline 1 & 0.400 & {$[0.288,0.478]$} & {$[0.215,0.600]$} & $\mathrm{H}$ & 54 & 0.088 & {$[0.043,0.139]$} & {$[0.023,0.237]$} & B \\
\hline 12 & 0.024 & {$[0.012,0.037]$} & {$[0.006,0.064]$} & B & 55 & 1.108 & {$[0.578,1.831]$} & {$[0.283,2.909]$} & B \\
\hline 13 & 0.050 & {$[0.024,0.078]$} & {$[0.013,0.134]$} & B & 56 & 2.279 & {$[0.584,2.976]$} & {$[0.118,4.780]$} & A \\
\hline 14 & 0.174 & {$[0.086,0.276]$} & {$[0.045,0.467]$} & B & 57 & 0.108 & {$[0.053,0.171]$} & {$[0.028,0.290]$} & B \\
\hline 15 & 0.864 & {$[0.443,1.406]$} & {$[0.220,2.263]$} & B & 58 & 0.004 & {$[0.002,0.007]$} & {$[0.001,0.012]$} & B \\
\hline 16 & 0.021 & {$[0.010,0.033]$} & {$[0.005,0.057]$} & B & 61 & 0.007 & {$[0.003,0.010]$} & {$[0.002,0.018]$} & B \\
\hline 25 & 0.014 & {$[0.007,0.022]$} & {$[0.004,0.038]$} & B & 62 & 0.011 & {$[0.005,0.017]$} & {$[0.003,0.030]$} & B \\
\hline 26 & 0.283 & {$[0.140,0.450]$} & {$[0.072,0.753]$} & B & 63 & 0.017 & {$[0.008,0.027]$} & {$[0.004,0.047]$} & B \\
\hline 27 & 2.099 & {$[0.391,2.702]$} & {$[0.045,4.559]$} & A & 64 & 0.003 & {$[0.001,0.005]$} & {$[0.001,0.008]$} & B \\
\hline 28 & 0.429 & {$[0.215,0.688]$} & {$[0.109,1.131]$} & B & 65 & 0.022 & {$[0.011,0.034]$} & {$[0.006,0.058]$} & B \\
\hline 29 & 1.449 & {$[0.768,2.416]$} & {$[0.371,3.771]$} & B & 66 & 0.010 & {$[0.005,0.016]$} & {$[0.003,0.028]$} & B \\
\hline 30 & 0.042 & {$[0.020,0.065]$} & {$[0.011,0.113]$} & B & 67 & 0.069 & {$[0.034,0.108]$} & {$[0.018,0.184]$} & B \\
\hline 31 & 0.011 & {$[0.005,0.017]$} & {$[0.003,0.030]$} & B & 68 & 0.012 & {$[0.006,0.019]$} & {$[0.003,0.033]$} & B \\
\hline 32 & 0.007 & {$[0.003,0.011]$} & {$[0.002,0.019]$} & B & 69 & 0.607 & {$[0.306,0.976]$} & {$[0.154,1.596]$} & B \\
\hline 39 & 1.806 & {$[0.991,3.079]$} & {$[0.464,4.659]$} & B & 70 & 0.034 & {$[0.017,0.053]$} & {$[0.009,0.091]$} & B \\
\hline 40 & 0.367 & {$[0.183,0.586]$} & {$[0.094,0.977]$} & B & 71 & 0.005 & {$[0.003,0.008]$} & {$[0.001,0.014]$} & B \\
\hline 41 & 2.949 & {$[1.318,3.869]$} & {$[0.574,5.595]$} & A & 74 & 0.003 & {$[0.001,0.005]$} & {$[0.001,0.008]$} & B \\
\hline 42 & 1.026 & {$[0.529,1.679]$} & {$[0.260,2.679]$} & B & 77 & 0.004 & {$[0.002,0.007]$} & {$[0.001,0.012]$} & B \\
\hline 43 & 0.313 & {$[0.155,0.498]$} & {$[0.080,0.832]$} & B & 79 & 0.003 & {$[0.002,0.005]$} & {$[0.001,0.009]$} & B \\
\hline 44 & 0.012 & {$[0.006,0.019]$} & {$[0.003,0.033]$} & B & 81 & 0.002 & {$[0.001,0.004]$} & {$[0.001,0.007]$} & B \\
\hline 50 & 0.099 & {$[0.049,0.155]$} & {$[0.026,0.267]$} & B & 83 & 0.006 & {$[0.003,0.010]$} & {$[0.002,0.017]$} & B \\
\hline 51 & 0.132 & {$[0.065,0.209]$} & {$[0.034,0.354]$} & B & 84 & 0.402 & {$[0.202,0.647]$} & {$[0.104,1.078]$} & B \\
\hline 52 & 0.049 & {$[0.024,0.077]$} & {$[0.013,0.134]$} & B & 85 & 0.027 & {$[0.013,0.043]$} & {$[0.007,0.073]$} & B \\
\hline 53 & 0.288 & {$[0.143,0.458]$} & {$[0.073,0.764]$} & B & & & & & \\
\hline
\end{tabular}


Table 11. Partial ionization cross sections $\left(10^{-20} \mathrm{~m}^{2}\right)$ for 3-methyl-1-pentene. TICS $/ 10^{-20} \mathrm{~m}^{2}=18.152 . M=84$, base peak is $\mathrm{m} / \mathrm{z} 55$.

\begin{tabular}{|c|c|c|c|c|c|c|c|c|c|}
\hline$m / z$ & PICS & $67 \%$ interval & $95 \%$ interval & unc. model & $m / z$ & PICS & $67 \%$ interval & $95 \%$ interval & unc. model \\
\hline 1 & 0.400 & {$[0.287,0.478]$} & {$[0.215,0.599]$} & $\mathrm{H}$ & 52 & 0.096 & {$[0.047,0.151]$} & {$[0.025,0.258]$} & B \\
\hline 15 & 0.347 & {$[0.174,0.554]$} & {$[0.089,0.923]$} & B & 53 & 0.495 & {$[0.248,0.791]$} & {$[0.126,1.314]$} & B \\
\hline 26 & 0.389 & {$[0.197,0.629]$} & {$[0.100,1.040]$} & B & 54 & 0.304 & {$[0.153,0.489]$} & {$[0.078,0.812]$} & B \\
\hline 27 & 2.083 & {$[1.169,3.599]$} & {$[0.532,5.317]$} & B & 55 & 2.638 & {$[0.949,3.443]$} & {$[0.305,5.181]$} & A \\
\hline 28 & 0.197 & {$[0.099,0.315]$} & {$[0.051,0.529]$} & B & 56 & 1.118 & {$[0.582,1.843]$} & {$[0.286,2.932]$} & B \\
\hline 29 & 1.429 & {$[0.760,2.387]$} & {$[0.365,3.706]$} & B & 57 & 0.071 & {$[0.035,0.112]$} & {$[0.018,0.192]$} & B \\
\hline 37 & 0.058 & {$[0.028,0.091]$} & {$[0.015,0.156]$} & B & 63 & 0.029 & {$[0.014,0.045]$} & {$[0.007,0.077]$} & B \\
\hline 38 & 0.188 & {$[0.093,0.299]$} & {$[0.048,0.507]$} & B & 65 & 0.043 & {$[0.021,0.068]$} & {$[0.011,0.117]$} & B \\
\hline 39 & 1.675 & {$[0.904,2.821]$} & {$[0.429,4.335]$} & B & 66 & 0.023 & {$[0.011,0.036]$} & {$[0.006,0.062]$} & B \\
\hline 40 & 0.300 & {$[0.150,0.480]$} & {$[0.077,0.803]$} & B & 67 & 0.119 & {$[0.059,0.188]$} & {$[0.030,0.318]$} & B \\
\hline 41 & 2.799 & {$[1.128,3.655]$} & {$[0.427,5.381]$} & A & 69 & 1.499 & {$[0.800,2.508]$} & {$[0.385,3.892]$} & B \\
\hline 42 & 0.643 & {$[0.326,1.040]$} & {$[0.163,1.693]$} & B & 70 & 0.081 & {$[0.039,0.126]$} & {$[0.021,0.217]$} & B \\
\hline 43 & 0.205 & {$[0.102,0.326]$} & {$[0.053,0.549]$} & B & 84 & 0.457 & {$[0.231,0.739]$} & {$[0.118,1.225]$} & B \\
\hline 50 & 0.183 & {$[0.090,0.290]$} & {$[0.047,0.489]$} & B & 85 & 0.030 & {$[0.014,0.046]$} & {$[0.008,0.080]$} & B \\
\hline 51 & 0.253 & {$[0.126,0.402]$} & {$[0.065,0.676]$} & B & & & & & \\
\hline
\end{tabular}

Table 12. Partial ionization cross sections $\left(10^{-20} \mathrm{~m}^{2}\right)$ for 4-methyl-1-pentene. TICS/10 $0^{-20} \mathrm{~m}^{2}=17.956 . M=84$, base peak is $\mathrm{m} / \mathrm{z} 43$.

\begin{tabular}{|c|c|c|c|c|c|c|c|c|c|}
\hline$m / z$ & PICS & $67 \%$ interval & $95 \%$ interval & unc. model & $m / z$ & PICS & $67 \%$ interval & $95 \%$ interval & unc. model \\
\hline 15 & 0.166 & {$[0.083,0.264]$} & {$[0.043,0.444]$} & B & 55 & 0.332 & {$[0.166,0.531]$} & {$[0.085,0.882]$} & B \\
\hline 27 & 1.571 & {$[0.846,2.650]$} & {$[0.399,4.064]$} & B & 57 & 0.082 & {$[0.040,0.130]$} & {$[0.021,0.220]$} & B \\
\hline 28 & 0.088 & {$[0.043,0.138]$} & {$[0.022,0.235]$} & B & 61 & 0.003 & {$[0.002,0.005]$} & {$[0.001,0.009]$} & B \\
\hline 29 & 0.300 & {$[0.150,0.478]$} & {$[0.077,0.798]$} & B & 62 & 0.009 & {$[0.004,0.014]$} & {$[0.002,0.025]$} & B \\
\hline 37 & 0.047 & {$[0.023,0.073]$} & {$[0.012,0.127]$} & B & 65 & 0.017 & {$[0.008,0.027]$} & {$[0.004,0.046]$} & B \\
\hline 38 & 0.114 & {$[0.056,0.180]$} & {$[0.029,0.304]$} & B & 66 & 0.005 & {$[0.003,0.009]$} & {$[0.001,0.015]$} & B \\
\hline 39 & 1.117 & {$[0.581,1.842]$} & {$[0.284,2.920]$} & B & 67 & 0.060 & {$[0.029,0.095]$} & {$[0.016,0.163]$} & B \\
\hline 40 & 0.207 & {$[0.102,0.327]$} & {$[0.052,0.545]$} & B & 68 & 0.013 & {$[0.006,0.020]$} & {$[0.003,0.034]$} & B \\
\hline 41 & 3.632 & {$[2.002,4.627]$} & {$[1.095,6.310]$} & A & 69 & 0.410 & {$[0.205,0.652]$} & {$[0.104,1.078]$} & B \\
\hline 42 & 1.633 & {$[0.881,2.753]$} & {$[0.418,4.225]$} & B & 70 & 0.023 & {$[0.011,0.036]$} & {$[0.006,0.062]$} & B \\
\hline 50 & 0.039 & {$[0.019,0.060]$} & {$[0.010,0.104]$} & B & 84 & 0.235 & {$[0.118,0.378]$} & {$[0.061,0.631]$} & B \\
\hline 51 & 0.054 & {$[0.026,0.084]$} & {$[0.014,0.145]$} & B & 85 & 0.016 & {$[0.008,0.025]$} & {$[0.004,0.043]$} & B \\
\hline 52 & 0.020 & {$[0.010,0.032]$} & {$[0.005,0.055]$} & $\mathrm{B}$ & & & & & \\
\hline
\end{tabular}


Table 13. Partial ionization cross sections $\left(10^{-20} \mathrm{~m}^{2}\right)$ for 2-methyl-2-pentene. TICS $/ 10^{-20} \mathrm{~m}^{2}=18.300 . M=84$, base peak is $m / z 41$.

\begin{tabular}{|c|c|c|c|c|c|c|c|c|c|}
\hline$m / z$ & PICS & $67 \%$ interval & $\mathbf{9 5 \%}$ interval & unc. model & $m / z$ & PICS & $67 \%$ interval & $95 \%$ interval & unc. model \\
\hline 1 & 0.400 & {$[0.288,0.478]$} & {$[0.215,0.599]$} & $\mathrm{H}$ & 56 & 0.633 & {$[0.322,1.027]$} & {$[0.162,1.680]$} & B \\
\hline 15 & 0.100 & {$[0.049,0.158]$} & {$[0.026,0.269]$} & B & 57 & 0.040 & {$[0.020,0.063]$} & {$[0.010,0.108]$} & B \\
\hline 26 & 0.079 & {$[0.038,0.123]$} & {$[0.020,0.211]$} & B & 58 & 0.012 & {$[0.006,0.018]$} & {$[0.003,0.032]$} & B \\
\hline 27 & 0.986 & {$[0.512,1.626]$} & {$[0.252,2.600]$} & B & 59 & 0.027 & {$[0.013,0.042]$} & {$[0.007,0.072]$} & B \\
\hline 28 & 0.190 & {$[0.094,0.302]$} & {$[0.049,0.507]$} & B & 61 & 0.007 & {$[0.003,0.011]$} & {$[0.002,0.019]$} & B \\
\hline 29 & 0.701 & {$[0.359,1.141]$} & {$[0.179,1.854]$} & B & 62 & 0.014 & {$[0.007,0.022]$} & {$[0.004,0.038]$} & B \\
\hline 30 & 0.016 & {$[0.008,0.025]$} & {$[0.004,0.042]$} & B & 63 & 0.024 & {$[0.011,0.037]$} & {$[0.006,0.064]$} & B \\
\hline 31 & 0.015 & {$[0.007,0.024]$} & {$[0.004,0.041]$} & B & 64 & 0.003 & {$[0.002,0.005]$} & {$[0.001,0.009]$} & B \\
\hline 32 & 0.007 & {$[0.004,0.012]$} & {$[0.002,0.020]$} & B & 65 & 0.041 & {$[0.020,0.064]$} & {$[0.010,0.110]$} & B \\
\hline 37 & 0.040 & {$[0.019,0.062]$} & {$[0.010,0.106]$} & B & 66 & 0.015 & {$[0.007,0.024]$} & {$[0.004,0.041]$} & B \\
\hline 38 & 0.109 & {$[0.053,0.171]$} & {$[0.028,0.291]$} & B & 67 & 0.183 & {$[0.090,0.291]$} & {$[0.047,0.490]$} & B \\
\hline 39 & 1.213 & {$[0.643,2.030]$} & {$[0.311,3.180]$} & B & 68 & 0.045 & {$[0.022,0.071]$} & {$[0.011,0.121]$} & B \\
\hline 40 & 0.257 & {$[0.127,0.408]$} & {$[0.065,0.680]$} & B & 69 & 2.724 & {$[1.051,3.554]$} & {$[0.350,5.221]$} & A \\
\hline 41 & 5.995 & {$[4.327,7.302]$} & {$[3.107,9.076]$} & A & 70 & 0.146 & {$[0.072,0.231]$} & {$[0.037,0.390]$} & B \\
\hline 42 & 0.930 & {$[0.482,1.531]$} & {$[0.239,2.453]$} & B & 71 & 0.010 & {$[0.005,0.016]$} & {$[0.003,0.027]$} & B \\
\hline 43 & 0.451 & {$[0.227,0.725]$} & {$[0.115,1.198]$} & B & 74 & 0.005 & {$[0.002,0.007]$} & {$[0.001,0.012]$} & B \\
\hline 44 & 0.017 & {$[0.008,0.026]$} & {$[0.004,0.045]$} & B & 77 & 0.009 & {$[0.004,0.014]$} & {$[0.002,0.024]$} & B \\
\hline 45 & 0.005 & {$[0.003,0.009]$} & {$[0.001,0.015]$} & B & 78 & 0.002 & {$[0.001,0.004]$} & {$[0.001,0.006]$} & B \\
\hline 49 & 0.010 & {$[0.005,0.015]$} & {$[0.003,0.027]$} & B & 79 & 0.009 & {$[0.004,0.014]$} & {$[0.002,0.025]$} & B \\
\hline 50 & 0.077 & {$[0.038,0.121]$} & {$[0.020,0.205]$} & B & 81 & 0.007 & {$[0.003,0.011]$} & {$[0.002,0.018]$} & B \\
\hline 51 & 0.117 & {$[0.058,0.185]$} & {$[0.030,0.312]$} & B & 82 & 0.005 & {$[0.002,0.007]$} & {$[0.001,0.012]$} & B \\
\hline 52 & 0.045 & {$[0.022,0.071]$} & {$[0.012,0.123]$} & B & 83 & 0.029 & {$[0.014,0.046]$} & {$[0.008,0.079]$} & B \\
\hline 53 & 0.322 & {$[0.161,0.515]$} & {$[0.082,0.861]$} & B & 84 & 0.964 & {$[0.500,1.587]$} & {$[0.246,2.533]$} & B \\
\hline 54 & 0.091 & {$[0.045,0.146]$} & {$[0.023,0.245]$} & B & 85 & 0.072 & {$[0.035,0.113]$} & {$[0.019,0.194]$} & B \\
\hline 55 & 1.101 & {$[0.575,1.825]$} & {$[0.280,2.893]$} & B & 86 & 0.002 & {$[0.001,0.004]$} & {$[0.001,0.006]$} & B \\
\hline
\end{tabular}

Table 14. Partial ionization cross sections $\left(10^{-20} \mathrm{~m}^{2}\right)$ for 3-methyl-2-pentene. TICS $/ 10^{-20} \mathrm{~m}^{2}=18.147 . M=84$, base peak is $\mathrm{m} / \mathrm{z} 41$.

\begin{tabular}{|c|c|c|c|c|c|c|c|c|c|}
\hline$m / z$ & PICS & $67 \%$ interval & $95 \%$ interval & unc. model & $m / \mathbf{z}$ & PICS & $67 \%$ interval & $95 \%$ interval & unc. model \\
\hline 1 & 0.168 & {$[0.083,0.265]$} & {$[0.043,0.448]$} & B & 54 & 0.154 & {$[0.076,0.244]$} & {$[0.040,0.414]$} & B \\
\hline 2 & 0.118 & {$[0.058,0.186]$} & {$[0.030,0.313]$} & B & 55 & 1.477 & {$[0.796,2.492]$} & {$[0.378,3.826]$} & B \\
\hline 12 & 0.011 & {$[0.005,0.017]$} & {$[0.003,0.030]$} & B & 56 & 0.693 & {$[0.352,1.121]$} & {$[0.177,1.823]$} & B \\
\hline 13 & 0.011 & {$[0.005,0.017]$} & {$[0.003,0.029]$} & B & 57 & 0.035 & {$[0.017,0.054]$} & {$[0.009,0.094]$} & B \\
\hline 14 & 0.031 & {$[0.015,0.049]$} & {$[0.008,0.084]$} & B & 58 & 0.003 & {$[0.001,0.005]$} & {$[0.001,0.008]$} & B \\
\hline 15 & 0.473 & {$[0.236,0.754]$} & {$[0.120,1.248]$} & B & 61 & 0.003 & {$[0.001,0.004]$} & {$[0.001,0.008]$} & B \\
\hline 16 & 0.009 & {$[0.005,0.015]$} & {$[0.002,0.026]$} & B & 62 & 0.005 & {$[0.003,0.009]$} & {$[0.001,0.015]$} & B \\
\hline 26 & 0.165 & {$[0.082,0.262]$} & {$[0.042,0.439]$} & B & 63 & 0.016 & {$[0.008,0.025]$} & {$[0.004,0.043]$} & B \\
\hline 27 & 2.159 & {$[1.222,3.694]$} & {$[0.556,5.418]$} & B & 64 & 0.003 & {$[0.001,0.004]$} & {$[0.001,0.007]$} & B \\
\hline 28 & 0.443 & {$[0.223,0.711]$} & {$[0.114,1.177]$} & B & 65 & 0.037 & {$[0.018,0.058]$} & {$[0.010,0.101]$} & B \\
\hline 29 & 1.166 & {$[0.612,1.934]$} & {$[0.298,3.046]$} & B & 66 & 0.019 & {$[0.009,0.030]$} & {$[0.005,0.052]$} & B \\
\hline 30 & 0.031 & {$[0.015,0.048]$} & {$[0.008,0.083]$} & B & 67 & 0.148 & {$[0.073,0.235]$} & {$[0.038,0.398]$} & B \\
\hline 31 & 0.006 & {$[0.003,0.009]$} & {$[0.002,0.016]$} & B & 68 & 0.031 & {$[0.015,0.049]$} & {$[0.008,0.083]$} & B \\
\hline 32 & 0.006 & {$[0.003,0.009]$} & {$[0.002,0.016]$} & B & 69 & 1.712 & {$[0.936,2.902]$} & {$[0.441,4.420]$} & B \\
\hline 33 & 0.006 & {$[0.003,0.009]$} & {$[0.001,0.016]$} & B & 70 & 0.090 & {$[0.044,0.142]$} & {$[0.023,0.241]$} & B \\
\hline 36 & 0.005 & {$[0.003,0.008]$} & {$[0.001,0.014]$} & B & 71 & 0.002 & {$[0.001,0.003]$} & {$[0.001,0.005]$} & B \\
\hline 37 & 0.026 & {$[0.013,0.040]$} & {$[0.007,0.070]$} & B & 72 & 0.002 & {$[0.001,0.003]$} & {$[0.000,0.005]$} & B \\
\hline 38 & 0.081 & {$[0.040,0.127]$} & {$[0.021,0.217]$} & B & 74 & 0.002 & {$[0.001,0.003]$} & {$[0.000,0.005]$} & B \\
\hline 39 & 1.374 & {$[0.731,2.293]$} & {$[0.352,3.575]$} & B & 75 & 0.002 & {$[0.001,0.003]$} & {$[0.000,0.005]$} & B \\
\hline 40 & 0.246 & {$[0.123,0.392]$} & {$[0.063,0.653]$} & B & 77 & 0.009 & {$[0.004,0.014]$} & {$[0.002,0.024]$} & B \\
\hline 41 & 4.707 & {$[3.034,5.822]$} & {$[1.962,7.523]$} & A & 78 & 0.002 & {$[0.001,0.003]$} & {$[0.000,0.005]$} & B \\
\hline 42 & 0.644 & {$[0.328,1.043]$} & {$[0.164,1.698]$} & B & 79 & 0.007 & {$[0.003,0.011]$} & {$[0.002,0.019]$} & B \\
\hline 43 & 0.346 & {$[0.172,0.552]$} & {$[0.088,0.922]$} & B & 80 & 0.002 & {$[0.001,0.003]$} & {$[0.000,0.005]$} & B \\
\hline 44 & 0.013 & {$[0.006,0.021]$} & {$[0.003,0.036]$} & B & 81 & 0.007 & {$[0.003,0.011]$} & {$[0.002,0.019]$} & B \\
\hline 49 & 0.004 & {$[0.002,0.006]$} & {$[0.001,0.011]$} & B & 82 & 0.002 & {$[0.001,0.003]$} & {$[0.000,0.005]$} & B \\
\hline 50 & 0.068 & {$[0.033,0.107]$} & {$[0.017,0.182]$} & B & 83 & 0.018 & {$[0.009,0.028]$} & {$[0.005,0.048]$} & B \\
\hline 51 & 0.159 & {$[0.079,0.252]$} & {$[0.041,0.425]$} & B & 84 & 0.674 & {$[0.344,1.094]$} & {$[0.173,1.783]$} & B \\
\hline 52 & 0.072 & {$[0.035,0.113]$} & {$[0.018,0.193]$} & B & 85 & 0.043 & {$[0.021,0.066]$} & {$[0.011,0.115]$} & B \\
\hline 53 & 0.401 & {$[0.200,0.639]$} & {$[0.102,1.060]$} & B & 86 & 0.002 & {$[0.001,0.003]$} & {$[0.000,0.005]$} & B \\
\hline
\end{tabular}


Table 15. Partial ionization cross sections $\left(10^{-20} \mathrm{~m}^{2}\right)$ for 4-methyl-2-pentene. TICS/10 $0^{-20} \mathrm{~m}^{2}=18.095 . M=84$, base peak is $m / z 41$.

\begin{tabular}{|c|c|c|c|c|c|c|c|c|c|}
\hline$m / z$ & PICS & $67 \%$ interval & $95 \%$ interval & unc. model & $m / z$ & PICS & $67 \%$ interval & $95 \%$ interval & unc. model \\
\hline 1 & 0.400 & {$[0.288,0.478]$} & {$[0.215,0.599]$} & $\mathrm{H}$ & 54 & 0.062 & {$[0.031,0.098]$} & {$[0.016,0.168]$} & B \\
\hline 2 & 0.071 & {$[0.035,0.112]$} & {$[0.018,0.192]$} & B & 55 & 0.425 & {$[0.213,0.681]$} & {$[0.109,1.132]$} & B \\
\hline 12 & 0.014 & {$[0.007,0.021]$} & {$[0.004,0.037]$} & B & 56 & 0.272 & {$[0.135,0.432]$} & {$[0.070,0.727]$} & B \\
\hline 13 & 0.026 & {$[0.013,0.041]$} & {$[0.007,0.070]$} & B & 57 & 0.015 & {$[0.007,0.024]$} & {$[0.004,0.042]$} & B \\
\hline 14 & 0.137 & {$[0.068,0.218]$} & {$[0.035,0.368]$} & B & 58 & 0.004 & {$[0.002,0.006]$} & {$[0.001,0.010]$} & B \\
\hline 15 & 0.946 & {$[0.493,1.561]$} & {$[0.242,2.487]$} & B & 60 & 0.004 & {$[0.002,0.005]$} & {$[0.001,0.010]$} & B \\
\hline 16 & 0.046 & {$[0.022,0.072]$} & {$[0.012,0.125]$} & B & 61 & 0.010 & {$[0.005,0.016]$} & {$[0.003,0.028]$} & B \\
\hline 26 & 0.200 & {$[0.098,0.316]$} & {$[0.051,0.537]$} & B & 62 & 0.017 & {$[0.008,0.026]$} & {$[0.004,0.045]$} & B \\
\hline 27 & 1.694 & {$[0.935,2.901]$} & {$[0.439,4.400]$} & B & 63 & 0.029 & {$[0.014,0.046]$} & {$[0.007,0.079]$} & B \\
\hline 28 & 0.260 & {$[0.128,0.414]$} & {$[0.066,0.698]$} & B & 64 & 0.006 & {$[0.003,0.010]$} & {$[0.002,0.017]$} & B \\
\hline 29 & 0.554 & {$[0.279,0.892]$} & {$[0.141,1.468]$} & B & 65 & 0.036 & {$[0.018,0.057]$} & {$[0.009,0.098]$} & B \\
\hline 30 & 0.015 & {$[0.007,0.023]$} & {$[0.004,0.040]$} & B & 66 & 0.020 & {$[0.010,0.032]$} & {$[0.005,0.055]$} & B \\
\hline 31 & 0.029 & {$[0.014,0.046]$} & {$[0.007,0.079]$} & B & 67 & 0.129 & {$[0.064,0.204]$} & {$[0.033,0.347]$} & B \\
\hline 32 & 0.021 & {$[0.010,0.033]$} & {$[0.005,0.057]$} & B & 68 & 0.046 & {$[0.022,0.072]$} & {$[0.012,0.124]$} & B \\
\hline 33 & 0.007 & {$[0.003,0.011]$} & {$[0.002,0.019]$} & B & 69 & 2.375 & {$[0.667,3.071]$} & {$[0.144,4.782]$} & A \\
\hline 34 & 0.007 & {$[0.003,0.011]$} & {$[0.002,0.019]$} & B & 70 & 0.122 & {$[0.061,0.193]$} & {$[0.031,0.324]$} & B \\
\hline 36 & 0.006 & {$[0.003,0.010]$} & {$[0.002,0.017]$} & B & 71 & 0.005 & {$[0.002,0.007]$} & {$[0.001,0.013]$} & B \\
\hline 37 & 0.088 & {$[0.043,0.139]$} & {$[0.023,0.235]$} & B & 73 & 0.002 & {$[0.001,0.003]$} & {$[0.001,0.006]$} & B \\
\hline 38 & 0.202 & {$[0.100,0.321]$} & {$[0.052,0.541]$} & B & 74 & 0.002 & {$[0.001,0.003]$} & {$[0.001,0.006]$} & B \\
\hline 39 & 1.660 & {$[0.910,2.837]$} & {$[0.427,4.322]$} & B & 75 & 0.002 & {$[0.001,0.003]$} & {$[0.001,0.006]$} & B \\
\hline 40 & 0.286 & {$[0.142,0.454]$} & {$[0.073,0.760]$} & B & 77 & 0.004 & {$[0.002,0.007]$} & {$[0.001,0.012]$} & B \\
\hline 41 & 5.708 & {$[4.048,6.971]$} & {$[2.842,8.702]$} & A & 78 & 0.002 & {$[0.001,0.003]$} & {$[0.001,0.006]$} & B \\
\hline 42 & 0.491 & {$[0.247,0.791]$} & {$[0.125,1.295]$} & B & 79 & 0.004 & {$[0.002,0.007]$} & {$[0.001,0.012]$} & B \\
\hline 43 & 0.398 & {$[0.200,0.639]$} & {$[0.102,1.062]$} & B & 80 & 0.002 & {$[0.001,0.003]$} & {$[0.001,0.006]$} & B \\
\hline 44 & 0.016 & {$[0.008,0.025]$} & {$[0.004,0.043]$} & B & 81 & 0.004 & {$[0.002,0.007]$} & {$[0.001,0.012]$} & B \\
\hline 48 & 0.005 & {$[0.002,0.008]$} & {$[0.001,0.013]$} & B & 82 & 0.002 & {$[0.001,0.003]$} & {$[0.001,0.006]$} & B \\
\hline 49 & 0.009 & {$[0.005,0.015]$} & {$[0.002,0.025]$} & B & 83 & 0.022 & {$[0.010,0.034]$} & {$[0.005,0.058]$} & B \\
\hline 50 & 0.078 & {$[0.038,0.123]$} & {$[0.020,0.210]$} & B & 84 & 0.597 & {$[0.303,0.965]$} & {$[0.154,1.588]$} & B \\
\hline 51 & 0.121 & {$[0.060,0.192]$} & {$[0.031,0.326]$} & B & 85 & 0.039 & {$[0.019,0.062]$} & {$[0.010,0.105]$} & B \\
\hline 52 & 0.044 & {$[0.021,0.069]$} & {$[0.011,0.119]$} & B & 86 & 0.002 & {$[0.001,0.003]$} & {$[0.001,0.006]$} & B \\
\hline 53 & 0.290 & {$[0.144,0.462]$} & {$[0.074,0.771]$} & B & & & & & \\
\hline
\end{tabular}

Table 16. Partial ionization cross sections $\left(10^{-20} \mathrm{~m}^{2}\right)$ for 2,3-dimethyl-1-butene. TICS/10-20 $\mathrm{m}^{2}=17.841 . M=84$, base peak is $m / z 41$.

\begin{tabular}{|c|c|c|c|c|c|c|c|c|c|}
\hline$m / z$ & PICS & $67 \%$ interval & $95 \%$ interval & unc. model & $m / z$ & PICS & $67 \%$ interval & $95 \%$ interval & unc. model \\
\hline 1 & 0.400 & {$[0.288,0.478]$} & {$[0.216,0.601]$} & $\mathrm{H}$ & 55 & 0.456 & {$[0.230,0.735]$} & {$[0.117,1.211]$} & B \\
\hline 15 & 0.119 & {$[0.059,0.188]$} & {$[0.031,0.321]$} & B & 56 & 0.342 & {$[0.171,0.547]$} & {$[0.087,0.910]$} & B \\
\hline 26 & 0.062 & {$[0.030,0.097]$} & {$[0.016,0.166]$} & B & 57 & 0.024 & {$[0.012,0.037]$} & {$[0.006,0.065]$} & B \\
\hline 27 & 0.853 & {$[0.438,1.393]$} & {$[0.218,2.252]$} & B & 61 & 0.004 & {$[0.002,0.007]$} & {$[0.001,0.012]$} & B \\
\hline 28 & 0.078 & {$[0.038,0.124]$} & {$[0.020,0.210]$} & B & 62 & 0.012 & {$[0.006,0.019]$} & {$[0.003,0.033]$} & B \\
\hline 29 & 0.342 & {$[0.170,0.545]$} & {$[0.087,0.910]$} & B & 63 & 0.020 & {$[0.010,0.031]$} & {$[0.005,0.054]$} & B \\
\hline 37 & 0.047 & {$[0.023,0.074]$} & {$[0.012,0.127]$} & B & 65 & 0.034 & {$[0.016,0.053]$} & {$[0.009,0.091]$} & B \\
\hline 38 & 0.121 & {$[0.060,0.191]$} & {$[0.031,0.324]$} & B & 66 & 0.011 & {$[0.005,0.017]$} & {$[0.003,0.029]$} & B \\
\hline 39 & 1.284 & {$[0.685,2.156]$} & {$[0.329,3.362]$} & B & 67 & 0.177 & {$[0.088,0.283]$} & {$[0.045,0.475]$} & B \\
\hline 40 & 0.260 & {$[0.129,0.413]$} & {$[0.067,0.698]$} & B & 68 & 0.033 & {$[0.016,0.052]$} & {$[0.008,0.089]$} & B \\
\hline 41 & 7.082 & {$[5.361,8.438]$} & {$[4.059,10.247]$} & A & 69 & 3.221 & {$[1.640,4.177]$} & {$[0.771,5.779]$} & A \\
\hline 42 & 0.615 & {$[0.311,0.994]$} & {$[0.157,1.631]$} & B & 70 & 0.173 & {$[0.085,0.274]$} & {$[0.044,0.464]$} & B \\
\hline 43 & 0.465 & {$[0.235,0.751]$} & {$[0.119,1.237]$} & B & 71 & 0.003 & {$[0.001,0.005]$} & {$[0.001,0.008]$} & B \\
\hline 44 & 0.013 & {$[0.006,0.021]$} & {$[0.003,0.036]$} & B & 77 & 0.003 & {$[0.001,0.004]$} & {$[0.001,0.007]$} & B \\
\hline 49 & 0.006 & {$[0.003,0.009]$} & {$[0.002,0.016]$} & B & 79 & 0.003 & {$[0.001,0.004]$} & {$[0.001,0.007]$} & B \\
\hline 50 & 0.062 & {$[0.031,0.098]$} & {$[0.016,0.169]$} & B & 81 & 0.003 & {$[0.001,0.004]$} & {$[0.001,0.007]$} & B \\
\hline 51 & 0.100 & {$[0.049,0.157]$} & {$[0.026,0.267]$} & B & 83 & 0.032 & {$[0.015,0.050]$} & {$[0.008,0.085]$} & B \\
\hline 52 & 0.038 & {$[0.018,0.059]$} & {$[0.010,0.102]$} & B & 84 & 0.927 & {$[0.481,1.529]$} & {$[0.235,2.433]$} & B \\
\hline 53 & 0.296 & {$[0.147,0.470]$} & {$[0.075,0.787]$} & B & 85 & 0.064 & {$[0.031,0.100]$} & {$[0.016,0.173]$} & B \\
\hline 54 & 0.056 & {$[0.028,0.089]$} & {$[0.015,0.152]$} & B & & & & & \\
\hline
\end{tabular}


Table 17. Partial ionization cross sections $\left(10^{-20} \mathrm{~m}^{2}\right)$ for 3,3-dimethyl-1-butene. TICS $/ 10^{-20} \mathrm{~m}^{2}=17.672 . M=84$, base peak is $\mathrm{m} / \mathrm{z} 69$.

\begin{tabular}{llllllllll}
\hline $\mathbf{m} / \mathbf{z}$ & $\mathbf{P I C S}$ & $\mathbf{6 7 \%}$ interval & $\mathbf{9 5 \%}$ interval & unc. model & $\mathbf{m} / \mathbf{z}$ & $\mathbf{P I C S}$ & $\mathbf{6 7 \%}$ interval & $\mathbf{9 5 \%}$ interval & unc. model \\
\hline 1 & 0.400 & {$[0.287,0.477]$} & {$[0.215,0.599]$} & $\mathrm{H}$ & 54 & 0.061 & {$[0.030,0.096]$} & {$[0.016,0.164]$} & $\mathrm{B}$ \\
14 & 0.017 & {$[0.008,0.026]$} & {$[0.004,0.045]$} & $\mathrm{B}$ & 55 & 0.376 & {$[0.189,0.604]$} & {$[0.096,1.005]$} & $\mathrm{B}$ \\
15 & 0.176 & {$[0.087,0.279]$} & {$[0.045,0.472]$} & $\mathrm{B}$ & 56 & 0.387 & {$[0.195,0.625]$} & {$[0.099,1.033]$} & $\mathrm{B}$ \\
26 & 0.111 & {$[0.054,0.175]$} & {$[0.028,0.298]$} & $\mathrm{B}$ & 57 & 0.392 & {$[0.196,0.625]$} & {$[0.100,1.043]$} & $\mathrm{B}$ \\
27 & 1.068 & {$[0.558,1.770]$} & {$[0.273,2.814]$} & $\mathrm{B}$ & 58 & 0.015 & {$[0.007,0.024]$} & {$[0.004,0.041]$} & $\mathrm{B}$ \\
28 & 0.064 & {$[0.031,0.101]$} & {$[0.016,0.172]$} & $\mathrm{B}$ & 61 & 0.009 & {$[0.004,0.014]$} & {$[0.002,0.025]$} & $\mathrm{B}$ \\
29 & 0.671 & {$[0.342,1.092]$} & {$[0.171,1.781]$} & $\mathrm{B}$ & 62 & 0.013 & {$[0.007,0.021]$} & {$[0.003,0.036]$} & $\mathrm{B}$ \\
30 & 0.010 & {$[0.005,0.016]$} & {$[0.003,0.027]$} & $\mathrm{B}$ & 63 & 0.022 & {$[0.011,0.034]$} & {$[0.006,0.058]$} & $\mathrm{B}$ \\
37 & 0.042 & {$[0.021,0.067]$} & {$[0.011,0.114]$} & $\mathrm{B}$ & 65 & 0.028 & {$[0.014,0.045]$} & {$[0.007,0.077]$} & $\mathrm{B}$ \\
38 & 0.123 & {$[0.061,0.195]$} & {$[0.032,0.330]$} & $\mathrm{B}$ & 66 & 0.008 & {$[0.004,0.012]$} & {$[0.002,0.021]$} & $\mathrm{B}$ \\
39 & 1.129 & {$[0.594,1.878]$} & {$[0.289,2.969]$} & $\mathrm{B}$ & 67 & 0.136 & {$[0.067,0.216]$} & {$[0.035,0.365]$} & $\mathrm{B}$ \\
40 & 0.188 & {$[0.093,0.299]$} & {$[0.048,0.503]$} & $\mathrm{B}$ & 68 & 0.029 & {$[0.014,0.045]$} & {$[0.007,0.078]$} & $\mathrm{B}$ \\
41 & 6.421 & {$[4.763,7.714]$} & {$[3.530,9.462]$} & $\mathrm{A}$ & 69 & 3.501 & {$[1.936,4.483]$} & {$[1.023,6.074]$} & $\mathrm{A}$ \\
42 & 0.435 & {$[0.219,0.700]$} & {$[0.112,1.165]$} & $\mathrm{B}$ & 70 & 0.205 & {$[0.101,0.323]$} & {$[0.052,0.547]$} & $\mathrm{B}$ \\
43 & 0.271 & {$[0.136,0.436]$} & {$[0.069,0.725]$} & $\mathrm{B}$ & 71 & 0.006 & {$[0.003,0.010]$} & {$[0.002,0.017]$} & $\mathrm{B}$ \\
44 & 0.007 & {$[0.003,0.011]$} & {$[0.002,0.019]$} & $\mathrm{B}$ & 77 & 0.003 & {$[0.001,0.004]$} & {$[0.001,0.008]$} & $\mathrm{B}$ \\
49 & 0.013 & {$[0.006,0.020]$} & {$[0.003,0.034]$} & $\mathrm{B}$ & 79 & 0.003 & {$[0.001,0.005]$} & {$[0.001,0.008]$} & $\mathrm{B}$ \\
50 & 0.086 & {$[0.042,0.135]$} & {$[0.022,0.233]$} & $\mathrm{B}$ & 83 & 0.012 & {$[0.006,0.018]$} & {$[0.003,0.031]$} & $\mathrm{B}$ \\
51 & 0.138 & {$[0.069,0.220]$} & {$[0.036,0.372]$} & $\mathrm{B}$ & 84 & 0.656 & {$[0.336,1.071]$} & {$[0.168,1.747]$} & $\mathrm{B}$ \\
52 & 0.041 & {$[0.020,0.065]$} & {$[0.011,0.111]$} & $\mathrm{B}$ & 85 & 0.043 & {$[0.021,0.068]$} & {$[0.011,0.117]$} & $\mathrm{B}$ \\
53 & 0.356 & {$[0.177,0.566]$} & {$[0.091,0.948]$} & $\mathrm{B}$ & & & & & \\
\hline
\end{tabular}

Table 18. Partial ionization cross sections $\left(10^{-20} \mathrm{~m}^{2}\right)$ for 2,3-dimethyl-2-butene. TICS $/ 10^{-20} \mathrm{~m}^{2}=18.571 . M=84$, base peak is $m / z 41$.

\begin{tabular}{|c|c|c|c|c|c|c|c|c|c|}
\hline$m / \mathbf{z}$ & PICS & $67 \%$ interval & $95 \%$ interval & unc. model & $m / \mathbf{z}$ & PICS & $67 \%$ interval & $95 \%$ interval & unc. model \\
\hline 1 & 0.400 & {$[0.288,0.478]$} & {$[0.216,0.600]$} & $\mathrm{H}$ & 53 & 0.379 & {$[0.189,0.604]$} & {$[0.097,1.006]$} & B \\
\hline 14 & 0.032 & {$[0.016,0.050]$} & {$[0.008,0.086]$} & B & 54 & 0.073 & {$[0.036,0.117]$} & {$[0.019,0.199]$} & B \\
\hline 15 & 0.333 & {$[0.165,0.528]$} & {$[0.085,0.888]$} & B & 55 & 0.432 & {$[0.216,0.690]$} & {$[0.110,1.146]$} & B \\
\hline 24 & 0.011 & {$[0.005,0.017]$} & {$[0.003,0.030]$} & B & 56 & 0.325 & {$[0.162,0.518]$} & {$[0.083,0.869]$} & B \\
\hline 26 & 0.095 & {$[0.047,0.151]$} & {$[0.024,0.255]$} & B & 57 & 0.034 & {$[0.017,0.053]$} & {$[0.009,0.092]$} & B \\
\hline 27 & 1.174 & {$[0.617,1.955]$} & {$[0.300,3.078]$} & B & 61 & 0.004 & {$[0.002,0.007]$} & {$[0.001,0.012]$} & B \\
\hline 28 & 0.040 & {$[0.019,0.063]$} & {$[0.010,0.108]$} & B & 62 & 0.008 & {$[0.004,0.013]$} & {$[0.002,0.023]$} & B \\
\hline 29 & 0.419 & {$[0.209,0.670]$} & {$[0.107,1.118]$} & B & 63 & 0.016 & {$[0.008,0.026]$} & {$[0.004,0.045]$} & B \\
\hline 37 & 0.056 & {$[0.027,0.088]$} & {$[0.014,0.152]$} & B & 65 & 0.027 & {$[0.013,0.042]$} & {$[0.007,0.072]$} & B \\
\hline 38 & 0.124 & {$[0.061,0.196]$} & {$[0.032,0.332]$} & B & 66 & 0.015 & {$[0.007,0.023]$} & {$[0.004,0.040]$} & B \\
\hline 39 & 1.371 & {$[0.734,2.310]$} & {$[0.351,3.585]$} & B & 67 & 0.200 & {$[0.099,0.319]$} & {$[0.051,0.538]$} & B \\
\hline 40 & 0.312 & {$[0.156,0.500]$} & {$[0.080,0.835]$} & B & 68 & 0.041 & {$[0.020,0.064]$} & {$[0.011,0.111]$} & B \\
\hline 41 & 7.263 & {$[5.489,8.679]$} & {$[4.139,10.552]$} & A & 69 & 2.976 & {$[1.304,3.890]$} & {$[0.502,5.562]$} & A \\
\hline 42 & 0.645 & {$[0.329,1.047]$} & {$[0.165,1.707]$} & B & 70 & 0.168 & {$[0.083,0.266]$} & {$[0.043,0.448]$} & B \\
\hline 43 & 0.484 & {$[0.244,0.781]$} & {$[0.123,1.282]$} & B & 71 & 0.003 & {$[0.001,0.005]$} & {$[0.001,0.008]$} & B \\
\hline 44 & 0.020 & {$[0.010,0.032]$} & {$[0.005,0.055]$} & B & 79 & 0.003 & {$[0.001,0.004]$} & {$[0.001,0.007]$} & B \\
\hline 49 & 0.006 & {$[0.003,0.009]$} & {$[0.002,0.016]$} & B & 81 & 0.003 & {$[0.001,0.004]$} & {$[0.001,0.007]$} & B \\
\hline 50 & 0.053 & {$[0.026,0.083]$} & {$[0.014,0.143]$} & B & 83 & 0.027 & {$[0.013,0.042]$} & {$[0.007,0.073]$} & B \\
\hline 51 & 0.091 & {$[0.045,0.143]$} & {$[0.023,0.244]$} & B & 84 & 0.811 & {$[0.418,1.335]$} & {$[0.207,2.146]$} & B \\
\hline 52 & 0.039 & {$[0.019,0.061]$} & {$[0.010,0.105]$} & B & 85 & 0.057 & {$[0.028,0.089]$} & {$[0.015,0.155]$} & B \\
\hline
\end{tabular}


Table 19. Partial ionization cross sections $\left(10^{-20} \mathrm{~m}^{2}\right)$ for 2-ethyl-1-butene. TICS $/ 10^{-20} \mathrm{~m}^{2}=18.169 . M=84$, base peak is $m / z 41$.

\begin{tabular}{|c|c|c|c|c|c|c|c|c|c|}
\hline$m / z$ & PICS & $67 \%$ interval & $95 \%$ interval & unc. model & $m / z$ & PICS & $67 \%$ interval & $95 \%$ interval & unc. model \\
\hline 1 & 0.400 & {$[0.288,0.478]$} & {$[0.215,0.600]$} & $\mathrm{H}$ & 55 & 1.828 & {$[1.006,3.119]$} & {$[0.471,4.722]$} & B \\
\hline 12 & 0.008 & {$[0.004,0.013]$} & {$[0.002,0.022]$} & B & 56 & 0.746 & {$[0.381,1.213]$} & {$[0.190,1.973]$} & B \\
\hline 13 & 0.008 & {$[0.004,0.012]$} & {$[0.002,0.021]$} & B & 57 & 0.035 & {$[0.017,0.054]$} & {$[0.009,0.093]$} & B \\
\hline 14 & 0.053 & {$[0.026,0.082]$} & {$[0.013,0.141]$} & B & 58 & 0.002 & {$[0.001,0.004]$} & {$[0.001,0.006]$} & B \\
\hline 15 & 0.498 & {$[0.251,0.804]$} & {$[0.126,1.320]$} & B & 61 & 0.002 & {$[0.001,0.003]$} & {$[0.001,0.006]$} & B \\
\hline 16 & 0.014 & {$[0.007,0.022]$} & {$[0.004,0.038]$} & B & 62 & 0.006 & {$[0.003,0.009]$} & {$[0.002,0.016]$} & B \\
\hline 25 & 0.005 & {$[0.002,0.008]$} & {$[0.001,0.014]$} & B & 63 & 0.014 & {$[0.007,0.021]$} & {$[0.003,0.037]$} & B \\
\hline 26 & 0.211 & {$[0.105,0.334]$} & {$[0.054,0.563]$} & B & 64 & 0.002 & {$[0.001,0.003]$} & {$[0.000,0.005]$} & B \\
\hline 27 & 2.417 & {$[0.707,3.131]$} & {$[0.170,4.886]$} & A & 65 & 0.025 & {$[0.012,0.040]$} & {$[0.007,0.069]$} & B \\
\hline 28 & 0.471 & {$[0.237,0.757]$} & {$[0.120,1.247]$} & B & 66 & 0.012 & {$[0.006,0.019]$} & {$[0.003,0.033]$} & B \\
\hline 29 & 1.597 & {$[0.865,2.706]$} & {$[0.407,4.125]$} & B & 67 & 0.085 & {$[0.042,0.134]$} & {$[0.022,0.227]$} & B \\
\hline 30 & 0.036 & {$[0.018,0.057]$} & {$[0.009,0.097]$} & B & 68 & 0.015 & {$[0.007,0.023]$} & {$[0.004,0.039]$} & B \\
\hline 31 & 0.004 & {$[0.002,0.007]$} & {$[0.001,0.012]$} & B & 69 & 1.399 & {$[0.742,2.336]$} & {$[0.358,3.651]$} & B \\
\hline 32 & 0.004 & {$[0.002,0.007]$} & {$[0.001,0.012]$} & B & 70 & 0.072 & {$[0.035,0.113]$} & {$[0.018,0.192]$} & B \\
\hline 33 & 0.004 & {$[0.002,0.006]$} & {$[0.001,0.011]$} & B & 71 & 0.001 & {$[0.001,0.002]$} & {$[0.000,0.004]$} & B \\
\hline 36 & 0.004 & {$[0.002,0.006]$} & {$[0.001,0.010]$} & B & 73 & 0.001 & {$[0.001,0.002]$} & {$[0.000,0.004]$} & B \\
\hline 37 & 0.030 & {$[0.015,0.047]$} & {$[0.008,0.082]$} & B & 74 & 0.001 & {$[0.001,0.002]$} & {$[0.000,0.004]$} & B \\
\hline 38 & 0.111 & {$[0.054,0.174]$} & {$[0.029,0.298]$} & B & 75 & 0.001 & {$[0.001,0.002]$} & {$[0.000,0.004]$} & B \\
\hline 39 & 1.415 & {$[0.753,2.364]$} & {$[0.364,3.690]$} & B & 76 & 0.001 & {$[0.001,0.002]$} & {$[0.000,0.004]$} & B \\
\hline 40 & 0.261 & {$[0.129,0.413]$} & {$[0.066,0.692]$} & B & 77 & 0.005 & {$[0.002,0.008]$} & {$[0.001,0.014]$} & B \\
\hline 41 & 3.438 & {$[1.812,4.459]$} & {$[0.927,6.155]$} & A & 78 & 0.001 & {$[0.001,0.002]$} & {$[0.000,0.004]$} & B \\
\hline 42 & 1.055 & {$[0.549,1.736]$} & {$[0.270,2.762]$} & B & 79 & 0.004 & {$[0.002,0.006]$} & {$[0.001,0.011]$} & B \\
\hline 43 & 0.325 & {$[0.162,0.518]$} & {$[0.084,0.867]$} & B & 80 & 0.001 & {$[0.001,0.002]$} & {$[0.000,0.003]$} & B \\
\hline 44 & 0.013 & {$[0.006,0.020]$} & {$[0.003,0.035]$} & B & 81 & 0.003 & {$[0.001,0.004]$} & {$[0.001,0.007]$} & B \\
\hline 49 & 0.006 & {$[0.003,0.009]$} & {$[0.001,0.015]$} & B & 82 & 0.001 & {$[0.001,0.002]$} & {$[0.000,0.004]$} & B \\
\hline 50 & 0.083 & {$[0.041,0.131]$} & {$[0.021,0.224]$} & B & 83 & 0.010 & {$[0.005,0.016]$} & {$[0.003,0.028]$} & B \\
\hline 51 & 0.160 & {$[0.079,0.255]$} & {$[0.041,0.429]$} & B & 84 & 0.551 & {$[0.278,0.886]$} & {$[0.141,1.463]$} & B \\
\hline 52 & 0.074 & {$[0.036,0.116]$} & {$[0.019,0.198]$} & B & 85 & 0.036 & {$[0.018,0.057]$} & {$[0.009,0.098]$} & B \\
\hline 53 & 0.391 & {$[0.195,0.622]$} & {$[0.100,1.031]$} & B & 86 & 0.001 & {$[0.001,0.002]$} & {$[0.000,0.003]$} & B \\
\hline 54 & 0.210 & {$[0.104,0.335]$} & {$[0.054,0.560]$} & B & & & & & \\
\hline
\end{tabular}

Table 20. Partial ionization cross sections $\left(10^{-20} \mathrm{~m}^{2}\right)$ for 1-heptene. TICS $/ 10^{-20} \mathrm{~m}^{2}=21.030 . M=98$, base peak is $\mathrm{m} / \mathrm{z} 56$.

\begin{tabular}{|c|c|c|c|c|c|c|c|c|c|}
\hline$m / z$ & PICS & $67 \%$ interval & $95 \%$ interval & unc. model & $m / z$ & PICS & $67 \%$ interval & $95 \%$ interval & unc. model \\
\hline 1 & 0.400 & {$[0.288,0.478]$} & {$[0.215,0.599]$} & $\mathrm{H}$ & 56 & 2.791 & {$[0.780,3.647]$} & {$[0.187,5.785]$} & A \\
\hline 15 & 0.083 & {$[0.040,0.128]$} & {$[0.021,0.221]$} & B & 57 & 0.839 & {$[0.426,1.354]$} & {$[0.213,2.202]$} & B \\
\hline 26 & 0.107 & {$[0.053,0.169]$} & {$[0.028,0.288]$} & B & 58 & 0.035 & {$[0.017,0.054]$} & {$[0.009,0.093]$} & B \\
\hline 27 & 1.343 & {$[0.696,2.211]$} & {$[0.340,3.513]$} & B & 61 & 0.003 & {$[0.001,0.004]$} & {$[0.001,0.007]$} & B \\
\hline 28 & 0.249 & {$[0.123,0.393]$} & {$[0.063,0.661]$} & B & 62 & 0.007 & {$[0.004,0.011]$} & {$[0.002,0.020]$} & B \\
\hline 29 & 2.779 & {$[0.789,3.650]$} & {$[0.180,5.764]$} & A & 63 & 0.012 & {$[0.006,0.019]$} & {$[0.003,0.032]$} & B \\
\hline 30 & 0.058 & {$[0.028,0.091]$} & {$[0.015,0.154]$} & B & 65 & 0.018 & {$[0.009,0.029]$} & {$[0.005,0.049]$} & B \\
\hline 36 & 0.004 & {$[0.002,0.007]$} & {$[0.001,0.012]$} & B & 66 & 0.007 & {$[0.003,0.010]$} & {$[0.002,0.018]$} & B \\
\hline 37 & 0.021 & {$[0.010,0.033]$} & {$[0.005,0.056]$} & B & 67 & 0.050 & {$[0.024,0.078]$} & {$[0.013,0.134]$} & B \\
\hline 38 & 0.073 & {$[0.035,0.114]$} & {$[0.019,0.195]$} & B & 68 & 0.077 & {$[0.037,0.120]$} & {$[0.020,0.206]$} & B \\
\hline 39 & 1.209 & {$[0.627,1.986]$} & {$[0.309,3.163]$} & B & 69 & 0.650 & {$[0.329,1.050]$} & {$[0.166,1.718]$} & B \\
\hline 40 & 0.190 & {$[0.094,0.301]$} & {$[0.048,0.505]$} & B & 70 & 0.901 & {$[0.460,1.463]$} & {$[0.229,2.369]$} & B \\
\hline 41 & 3.679 & {$[1.754,4.813]$} & {$[0.800,6.836]$} & A & 71 & 0.049 & {$[0.024,0.077]$} & {$[0.013,0.133]$} & B \\
\hline 42 & 2.043 & {$[1.104,3.442]$} & {$[0.525,5.296]$} & B & 77 & 0.007 & {$[0.003,0.011]$} & {$[0.002,0.018]$} & B \\
\hline 43 & 0.579 & {$[0.290,0.926]$} & {$[0.147,1.534]$} & B & 78 & 0.002 & {$[0.001,0.003]$} & {$[0.000,0.004]$} & B \\
\hline 44 & 0.018 & {$[0.009,0.028]$} & {$[0.005,0.048]$} & B & 79 & 0.005 & {$[0.002,0.007]$} & {$[0.001,0.013]$} & B \\
\hline 49 & 0.003 & {$[0.002,0.005]$} & {$[0.001,0.009]$} & B & 81 & 0.004 & {$[0.002,0.007]$} & {$[0.001,0.012]$} & B \\
\hline 50 & 0.047 & {$[0.023,0.074]$} & {$[0.012,0.127]$} & B & 82 & 0.001 & {$[0.001,0.002]$} & {$[0.000,0.004]$} & B \\
\hline 51 & 0.071 & {$[0.035,0.111]$} & {$[0.018,0.190]$} & B & 83 & 0.057 & {$[0.028,0.091]$} & {$[0.015,0.155]$} & B \\
\hline 52 & 0.030 & {$[0.015,0.047]$} & {$[0.008,0.081]$} & B & 84 & 0.004 & {$[0.002,0.006]$} & {$[0.001,0.011]$} & B \\
\hline 53 & 0.193 & {$[0.095,0.305]$} & {$[0.049,0.511]$} & B & 97 & 0.001 & {$[0.001,0.002]$} & {$[0.000,0.003]$} & B \\
\hline 54 & 0.221 & {$[0.108,0.348]$} & {$[0.056,0.590]$} & B & 98 & 0.172 & {$[0.085,0.272]$} & {$[0.044,0.462]$} & B \\
\hline 55 & 1.926 & {$[1.034,3.238]$} & {$[0.494,5.007]$} & B & 99 & 0.014 & {$[0.007,0.021]$} & {$[0.004,0.037]$} & B \\
\hline
\end{tabular}


Table 21. Partial ionization cross sections $\left(10^{-20} \mathrm{~m}^{2}\right)$ for 2-heptene. TICS $/ 10^{-20} \mathrm{~m}^{2}=21.308 . M=98$, base peak is $\mathrm{m} / \mathrm{z} 55$.

\begin{tabular}{|c|c|c|c|c|c|c|c|c|c|}
\hline$m / z$ & PICS & $67 \%$ interval & $95 \%$ interval & unc. model & $m / z$ & PICS & $67 \%$ interval & $95 \%$ interval & unc. model \\
\hline 1 & 0.400 & {$[0.289,0.479]$} & {$[0.214,0.599]$} & $\mathrm{H}$ & 62 & 0.015 & {$[0.007,0.023]$} & {$[0.004,0.040]$} & B \\
\hline 14 & 0.009 & {$[0.005,0.015]$} & {$[0.002,0.025]$} & B & 63 & 0.023 & {$[0.011,0.036]$} & {$[0.006,0.062]$} & B \\
\hline 15 & 0.126 & {$[0.062,0.199]$} & {$[0.032,0.337]$} & B & 64 & 0.003 & {$[0.001,0.004]$} & {$[0.001,0.008]$} & B \\
\hline 26 & 0.141 & {$[0.069,0.221]$} & {$[0.036,0.377]$} & B & 65 & 0.035 & {$[0.017,0.055]$} & {$[0.009,0.096]$} & B \\
\hline 27 & 1.644 & {$[0.873,2.753]$} & {$[0.419,4.285]$} & B & 66 & 0.016 & {$[0.008,0.025]$} & {$[0.004,0.043]$} & B \\
\hline 28 & 0.225 & {$[0.113,0.361]$} & {$[0.058,0.604]$} & B & 67 & 0.131 & {$[0.065,0.208]$} & {$[0.034,0.355]$} & B \\
\hline 29 & 1.308 & {$[0.679,2.151]$} & {$[0.333,3.421]$} & B & 68 & 0.092 & {$[0.045,0.145]$} & {$[0.024,0.248]$} & B \\
\hline 30 & 0.029 & {$[0.014,0.046]$} & {$[0.007,0.078]$} & B & 69 & 1.203 & {$[0.622,1.975]$} & {$[0.307,3.167]$} & B \\
\hline 37 & 0.040 & {$[0.019,0.062]$} & {$[0.010,0.107]$} & B & 70 & 0.412 & {$[0.205,0.655]$} & {$[0.104,1.085]$} & B \\
\hline 38 & 0.101 & {$[0.049,0.158]$} & {$[0.026,0.269]$} & B & 71 & 0.026 & {$[0.013,0.041]$} & {$[0.007,0.070]$} & B \\
\hline 39 & 1.309 & {$[0.682,2.165]$} & {$[0.333,3.435]$} & B & 72 & 0.002 & {$[0.001,0.004]$} & {$[0.001,0.006]$} & B \\
\hline 40 & 0.222 & {$[0.110,0.353]$} & {$[0.056,0.590]$} & B & 74 & 0.002 & {$[0.001,0.003]$} & {$[0.001,0.006]$} & B \\
\hline 41 & 3.379 & {$[1.412,4.450]$} & {$[0.552,6.506]$} & A & 77 & 0.014 & {$[0.007,0.022]$} & {$[0.004,0.038]$} & B \\
\hline 42 & 0.859 & {$[0.437,1.395]$} & {$[0.217,2.259]$} & B & 78 & 0.004 & {$[0.002,0.006]$} & {$[0.001,0.011]$} & B \\
\hline 43 & 0.894 & {$[0.457,1.456]$} & {$[0.228,2.361]$} & B & 79 & 0.015 & {$[0.007,0.023]$} & {$[0.004,0.041]$} & B \\
\hline 44 & 0.030 & {$[0.015,0.047]$} & {$[0.008,0.081]$} & B & 80 & 0.002 & {$[0.001,0.003]$} & {$[0.000,0.005]$} & B \\
\hline 45 & 0.013 & {$[0.006,0.020]$} & {$[0.003,0.034]$} & B & 81 & 0.021 & {$[0.010,0.033]$} & {$[0.005,0.057]$} & B \\
\hline 49 & 0.012 & {$[0.006,0.018]$} & {$[0.003,0.031]$} & B & 82 & 0.005 & {$[0.002,0.008]$} & {$[0.001,0.014]$} & B \\
\hline 50 & 0.068 & {$[0.033,0.106]$} & {$[0.017,0.181]$} & B & 83 & 0.069 & {$[0.034,0.109]$} & {$[0.018,0.186]$} & B \\
\hline 51 & 0.114 & {$[0.056,0.180]$} & {$[0.029,0.307]$} & B & 84 & 0.005 & {$[0.002,0.007]$} & {$[0.001,0.013]$} & B \\
\hline 52 & 0.050 & {$[0.025,0.079]$} & {$[0.013,0.136]$} & B & 85 & 0.002 & {$[0.001,0.002]$} & {$[0.000,0.004]$} & B \\
\hline 53 & 0.352 & {$[0.177,0.567]$} & {$[0.090,0.944]$} & B & 95 & 0.001 & {$[0.001,0.002]$} & {$[0.000,0.004]$} & B \\
\hline 54 & 0.404 & {$[0.202,0.647]$} & {$[0.103,1.071]$} & B & 96 & 0.001 & {$[0.001,0.002]$} & {$[0.000,0.004]$} & B \\
\hline 55 & 3.410 & {$[1.447,4.500]$} & {$[0.569,6.548]$} & A & 97 & 0.006 & {$[0.003,0.009]$} & {$[0.002,0.016]$} & B \\
\hline 56 & 3.019 & {$[1.023,3.981]$} & {$[0.308,6.073]$} & A & 98 & 0.647 & {$[0.327,1.043]$} & {$[0.166,1.719]$} & B \\
\hline 57 & 0.324 & {$[0.161,0.514]$} & {$[0.082,0.857]$} & B & 99 & 0.054 & {$[0.026,0.084]$} & {$[0.014,0.143]$} & B \\
\hline 58 & 0.013 & {$[0.006,0.020]$} & {$[0.003,0.035]$} & B & 100 & 0.001 & {$[0.001,0.002]$} & {$[0.000,0.004]$} & B \\
\hline 61 & 0.006 & {$[0.003,0.009]$} & {$[0.002,0.016]$} & B & & & & & \\
\hline
\end{tabular}


Table 22. Partial ionization cross sections $\left(10^{-20} \mathrm{~m}^{2}\right)$ for 3-heptene. TICS $/ 10^{-20} \mathrm{~m}^{2}=20.454 . M=98$, base peak is $\mathrm{m} / \mathrm{z} 41$.

\begin{tabular}{|c|c|c|c|c|c|c|c|c|c|}
\hline$m / z$ & PICS & $67 \%$ interval & $95 \%$ interval & unc. model & $m / z$ & PICS & $67 \%$ interval & $95 \%$ interval & unc. model \\
\hline 1 & 0.400 & {$[0.288,0.478]$} & {$[0.216,0.600]$} & $\mathrm{H}$ & 60 & 0.003 & {$[0.001,0.005]$} & {$[0.001,0.008]$} & B \\
\hline 12 & 0.010 & {$[0.005,0.016]$} & {$[0.003,0.028]$} & B & 61 & 0.006 & {$[0.003,0.009]$} & {$[0.002,0.016]$} & B \\
\hline 13 & 0.019 & {$[0.009,0.030]$} & {$[0.005,0.052]$} & B & 62 & 0.014 & {$[0.007,0.023]$} & {$[0.004,0.039]$} & B \\
\hline 14 & 0.075 & {$[0.037,0.117]$} & {$[0.019,0.199]$} & B & 63 & 0.028 & {$[0.014,0.044]$} & {$[0.007,0.077]$} & B \\
\hline 15 & 0.538 & {$[0.270,0.863]$} & {$[0.137,1.427]$} & B & 64 & 0.006 & {$[0.003,0.009]$} & {$[0.001,0.015]$} & B \\
\hline 16 & 0.009 & {$[0.004,0.013]$} & {$[0.002,0.023]$} & B & 65 & 0.041 & {$[0.020,0.064]$} & {$[0.010,0.109]$} & B \\
\hline 24 & 0.007 & {$[0.003,0.010]$} & {$[0.002,0.018]$} & B & 66 & 0.019 & {$[0.009,0.029]$} & {$[0.005,0.050]$} & B \\
\hline 25 & 0.013 & {$[0.006,0.020]$} & {$[0.003,0.035]$} & B & 67 & 0.129 & {$[0.064,0.204]$} & {$[0.033,0.346]$} & B \\
\hline 26 & 0.279 & {$[0.138,0.442]$} & {$[0.071,0.738]$} & B & 68 & 0.078 & {$[0.039,0.124]$} & {$[0.020,0.212]$} & B \\
\hline 27 & 2.291 & {$[1.287,3.933]$} & {$[0.585,5.781]$} & B & 69 & 1.378 & {$[0.725,2.286]$} & {$[0.351,3.590]$} & B \\
\hline 28 & 0.446 & {$[0.223,0.712]$} & {$[0.113,1.175]$} & B & 70 & 0.303 & {$[0.151,0.484]$} & {$[0.077,0.809]$} & B \\
\hline 29 & 1.134 & {$[0.591,1.871]$} & {$[0.289,2.976]$} & B & 71 & 0.014 & {$[0.007,0.022]$} & {$[0.004,0.038]$} & B \\
\hline 30 & 0.029 & {$[0.014,0.045]$} & {$[0.007,0.077]$} & B & 73 & 0.002 & {$[0.001,0.003]$} & {$[0.001,0.006]$} & B \\
\hline 31 & 0.006 & {$[0.003,0.009]$} & {$[0.001,0.015]$} & B & 75 & 0.002 & {$[0.001,0.003]$} & {$[0.001,0.006]$} & B \\
\hline 36 & 0.005 & {$[0.002,0.008]$} & {$[0.001,0.014]$} & B & 76 & 0.002 & {$[0.001,0.003]$} & {$[0.001,0.006]$} & B \\
\hline 37 & 0.049 & {$[0.024,0.077]$} & {$[0.012,0.131]$} & B & 77 & 0.014 & {$[0.007,0.022]$} & {$[0.004,0.037]$} & B \\
\hline 38 & 0.143 & {$[0.070,0.225]$} & {$[0.037,0.383]$} & B & 78 & 0.004 & {$[0.002,0.006]$} & {$[0.001,0.010]$} & B \\
\hline 39 & 1.499 & {$[0.801,2.519]$} & {$[0.384,3.907]$} & B & 79 & 0.013 & {$[0.006,0.020]$} & {$[0.003,0.036]$} & B \\
\hline 40 & 0.234 & {$[0.116,0.371]$} & {$[0.060,0.624]$} & B & 80 & 0.002 & {$[0.001,0.003]$} & {$[0.000,0.005]$} & B \\
\hline 41 & 4.486 & {$[2.632,5.643]$} & {$[1.526,7.502]$} & A & 81 & 0.016 & {$[0.008,0.024]$} & {$[0.004,0.042]$} & B \\
\hline 42 & 0.765 & {$[0.387,1.234]$} & {$[0.194,2.010]$} & B & 82 & 0.002 & {$[0.001,0.003]$} & {$[0.000,0.005]$} & B \\
\hline 43 & 0.551 & {$[0.279,0.889]$} & {$[0.140,1.456]$} & B & 83 & 0.052 & {$[0.025,0.082]$} & {$[0.013,0.140]$} & B \\
\hline 44 & 0.025 & {$[0.012,0.040]$} & {$[0.006,0.068]$} & B & 84 & 0.003 & {$[0.001,0.005]$} & {$[0.001,0.008]$} & B \\
\hline 45 & 0.004 & {$[0.002,0.006]$} & {$[0.001,0.011]$} & B & 85 & 0.001 & {$[0.001,0.002]$} & {$[0.000,0.004]$} & B \\
\hline 46 & 0.004 & {$[0.002,0.006]$} & {$[0.001,0.011]$} & B & 86 & 0.001 & {$[0.001,0.002]$} & {$[0.000,0.004]$} & B \\
\hline 49 & 0.008 & {$[0.004,0.012]$} & {$[0.002,0.021]$} & B & 87 & 0.001 & {$[0.001,0.002]$} & {$[0.000,0.004]$} & B \\
\hline 50 & 0.082 & {$[0.040,0.129]$} & {$[0.021,0.221]$} & B & 89 & 0.001 & {$[0.001,0.002]$} & {$[0.000,0.004]$} & B \\
\hline 51 & 0.139 & {$[0.069,0.220]$} & {$[0.036,0.371]$} & B & 91 & 0.001 & {$[0.001,0.002]$} & {$[0.000,0.004]$} & B \\
\hline 52 & 0.061 & {$[0.030,0.095]$} & {$[0.015,0.161]$} & B & 95 & 0.001 & {$[0.001,0.002]$} & {$[0.000,0.004]$} & B \\
\hline 53 & 0.302 & {$[0.150,0.481]$} & {$[0.077,0.805]$} & B & 96 & 0.001 & {$[0.001,0.002]$} & {$[0.000,0.004]$} & B \\
\hline 54 & 0.237 & {$[0.118,0.378]$} & {$[0.061,0.635]$} & B & 97 & 0.001 & {$[0.001,0.002]$} & {$[0.000,0.004]$} & B \\
\hline 55 & 1.606 & {$[0.859,2.695]$} & {$[0.412,4.189]$} & B & 98 & 0.411 & {$[0.205,0.653]$} & {$[0.105,1.087]$} & B \\
\hline 56 & 2.180 & {$[1.209,3.709]$} & {$[0.559,5.544]$} & B & 99 & 0.032 & {$[0.016,0.051]$} & {$[0.008,0.087]$} & B \\
\hline 57 & 0.226 & {$[0.112,0.360]$} & {$[0.058,0.605]$} & B & 100 & 0.001 & {$[0.001,0.002]$} & {$[0.000,0.004]$} & B \\
\hline 58 & 0.006 & {$[0.003,0.010]$} & {$[0.002,0.017]$} & B & & & & & \\
\hline
\end{tabular}


Table 23. Partial ionization cross sections $\left(10^{-20} \mathrm{~m}^{2}\right)$ for 2-methyl-1-hexene. TICS $/ 10^{-20} \mathrm{~m}^{2}=21.242 . M=98$, base peak is $\mathrm{m} / \mathrm{z} 56$.

\begin{tabular}{|c|c|c|c|c|c|c|c|c|c|}
\hline$m / z$ & PICS & $67 \%$ interval & $95 \%$ interval & unc. model & $m / z$ & PICS & $67 \%$ interval & $95 \%$ interval & unc. model \\
\hline 1 & 0.400 & {$[0.288,0.478]$} & {$[0.215,0.600]$} & $\mathrm{H}$ & 55 & 1.214 & {$[0.633,2.006]$} & {$[0.310,3.181]$} & B \\
\hline 15 & 0.128 & {$[0.063,0.201]$} & {$[0.033,0.342]$} & B & 56 & 6.722 & {$[4.771,8.192]$} & {$[3.378,10.243]$} & A \\
\hline 26 & 0.078 & {$[0.038,0.123]$} & {$[0.020,0.209]$} & B & 57 & 0.691 & {$[0.350,1.119]$} & {$[0.175,1.822]$} & B \\
\hline 27 & 1.472 & {$[0.780,2.458]$} & {$[0.378,3.855]$} & B & 58 & 0.026 & {$[0.012,0.040]$} & {$[0.007,0.070]$} & B \\
\hline 28 & 0.306 & {$[0.153,0.489]$} & {$[0.079,0.819]$} & B & 62 & 0.006 & {$[0.003,0.009]$} & {$[0.002,0.016]$} & B \\
\hline 29 & 1.065 & {$[0.554,1.757]$} & {$[0.274,2.816]$} & B & 63 & 0.012 & {$[0.006,0.018]$} & {$[0.003,0.031]$} & B \\
\hline 30 & 0.023 & {$[0.011,0.036]$} & {$[0.006,0.063]$} & B & 65 & 0.017 & {$[0.008,0.026]$} & {$[0.004,0.045]$} & B \\
\hline 37 & 0.020 & {$[0.010,0.031]$} & {$[0.005,0.053]$} & B & 66 & 0.005 & {$[0.003,0.008]$} & {$[0.001,0.015]$} & B \\
\hline 38 & 0.068 & {$[0.033,0.108]$} & {$[0.017,0.184]$} & B & 67 & 0.079 & {$[0.039,0.125]$} & {$[0.020,0.213]$} & B \\
\hline 39 & 1.002 & {$[0.516,1.641]$} & {$[0.255,2.645]$} & B & 68 & 0.031 & {$[0.015,0.048]$} & {$[0.008,0.084]$} & B \\
\hline 40 & 0.270 & {$[0.133,0.427]$} & {$[0.069,0.720]$} & B & 69 & 0.498 & {$[0.249,0.797]$} & {$[0.127,1.324]$} & B \\
\hline 41 & 4.264 & {$[2.374,5.461]$} & {$[1.279,7.403]$} & A & 70 & 0.608 & {$[0.306,0.977]$} & {$[0.156,1.619]$} & B \\
\hline 42 & 0.501 & {$[0.252,0.805]$} & {$[0.128,1.337]$} & B & 71 & 0.043 & {$[0.021,0.067]$} & {$[0.011,0.116]$} & B \\
\hline 43 & 1.009 & {$[0.518,1.647]$} & {$[0.257,2.657]$} & B & 77 & 0.008 & {$[0.004,0.013]$} & {$[0.002,0.022]$} & B \\
\hline 44 & 0.034 & {$[0.017,0.054]$} & {$[0.009,0.093]$} & B & 79 & 0.011 & {$[0.006,0.018]$} & {$[0.003,0.031]$} & B \\
\hline 50 & 0.046 & {$[0.022,0.071]$} & {$[0.012,0.122]$} & B & 81 & 0.014 & {$[0.007,0.022]$} & {$[0.004,0.038]$} & B \\
\hline 51 & 0.060 & {$[0.029,0.093]$} & {$[0.015,0.161]$} & B & 83 & 0.036 & {$[0.018,0.057]$} & {$[0.009,0.097]$} & B \\
\hline 52 & 0.029 & {$[0.014,0.046]$} & {$[0.008,0.078]$} & B & 84 & 0.006 & {$[0.003,0.010]$} & {$[0.002,0.017]$} & B \\
\hline 53 & 0.228 & {$[0.113,0.363]$} & {$[0.059,0.613]$} & B & 98 & 0.093 & {$[0.046,0.147]$} & {$[0.024,0.250]$} & B \\
\hline 54 & 0.111 & {$[0.055,0.176]$} & {$[0.029,0.298]$} & B & 99 & 0.009 & {$[0.004,0.014]$} & {$[0.002,0.024]$} & B \\
\hline
\end{tabular}

Table 24. Partial ionization cross sections $\left(10^{-20} \mathrm{~m}^{2}\right)$ for 3-methyl-1-hexene. TICS $/ 10^{-20} \mathrm{~m}^{2}=21.178 . M=98$, base peak is $\mathrm{m} / \mathrm{z} 55$.

\begin{tabular}{|c|c|c|c|c|c|c|c|c|c|}
\hline$m / z$ & PICS & $67 \%$ interval & $95 \%$ interval & unc. model & $m / \mathbf{z}$ & PICS & $67 \%$ interval & $95 \%$ interval & unc. model \\
\hline 1 & 0.400 & {$[0.289,0.479]$} & {$[0.216,0.600]$} & $\mathrm{H}$ & 58 & 0.003 & {$[0.002,0.005]$} & {$[0.001,0.009]$} & B \\
\hline 14 & 0.010 & {$[0.005,0.016]$} & {$[0.003,0.027]$} & B & 61 & 0.003 & {$[0.002,0.005]$} & {$[0.001,0.009]$} & B \\
\hline 15 & 0.107 & {$[0.052,0.168]$} & {$[0.027,0.286]$} & B & 62 & 0.010 & {$[0.005,0.015]$} & {$[0.002,0.026]$} & B \\
\hline 26 & 0.117 & {$[0.058,0.186]$} & {$[0.030,0.315]$} & B & 63 & 0.015 & {$[0.007,0.024]$} & {$[0.004,0.042]$} & B \\
\hline 27 & 1.564 & {$[0.834,2.618]$} & {$[0.402,4.076]$} & B & 65 & 0.029 & {$[0.014,0.046]$} & {$[0.008,0.079]$} & B \\
\hline 28 & 0.118 & {$[0.058,0.186]$} & {$[0.030,0.317]$} & B & 66 & 0.012 & {$[0.006,0.018]$} & {$[0.003,0.031]$} & B \\
\hline 29 & 1.224 & {$[0.638,2.020]$} & {$[0.313,3.216]$} & B & 67 & 0.152 & {$[0.075,0.241]$} & {$[0.039,0.407]$} & B \\
\hline 30 & 0.025 & {$[0.012,0.039]$} & {$[0.006,0.067]$} & B & 68 & 0.085 & {$[0.042,0.134]$} & {$[0.022,0.230]$} & B \\
\hline 37 & 0.027 & {$[0.013,0.042]$} & {$[0.007,0.073]$} & B & 69 & 2.121 & {$[1.165,3.606]$} & {$[0.548,5.467]$} & B \\
\hline 38 & 0.067 & {$[0.033,0.107]$} & {$[0.017,0.182]$} & B & 70 & 1.073 & {$[0.551,1.750]$} & {$[0.273,2.817]$} & B \\
\hline 39 & 1.084 & {$[0.558,1.774]$} & {$[0.276,2.851]$} & B & 71 & 0.059 & {$[0.029,0.092]$} & {$[0.015,0.159]$} & B \\
\hline 40 & 0.170 & {$[0.084,0.269]$} & {$[0.044,0.454]$} & B & 72 & 0.002 & {$[0.001,0.004]$} & {$[0.001,0.007]$} & B \\
\hline 41 & 3.539 & {$[1.605,4.610]$} & {$[0.697,6.608]$} & A & 74 & 0.002 & {$[0.001,0.004]$} & {$[0.001,0.006]$} & B \\
\hline 42 & 0.648 & {$[0.328,1.047]$} & {$[0.166,1.723]$} & B & 77 & 0.013 & {$[0.006,0.020]$} & {$[0.003,0.035]$} & B \\
\hline 43 & 1.006 & {$[0.517,1.644]$} & {$[0.256,2.638]$} & B & 78 & 0.002 & {$[0.001,0.003]$} & {$[0.001,0.006]$} & B \\
\hline 44 & 0.041 & {$[0.020,0.065]$} & {$[0.011,0.113]$} & B & 79 & 0.014 & {$[0.007,0.022]$} & {$[0.004,0.038]$} & B \\
\hline 49 & 0.008 & {$[0.004,0.013]$} & {$[0.002,0.022]$} & B & 80 & 0.002 & {$[0.001,0.003]$} & {$[0.001,0.005]$} & B \\
\hline 50 & 0.065 & {$[0.032,0.102]$} & {$[0.017,0.173]$} & B & 81 & 0.019 & {$[0.009,0.029]$} & {$[0.005,0.051]$} & B \\
\hline 51 & 0.107 & {$[0.053,0.169]$} & {$[0.027,0.286]$} & B & 82 & 0.013 & {$[0.006,0.020]$} & {$[0.003,0.035]$} & B \\
\hline 52 & 0.047 & {$[0.023,0.073]$} & {$[0.012,0.125]$} & B & 83 & 0.187 & {$[0.093,0.299]$} & {$[0.048,0.503]$} & B \\
\hline 53 & 0.337 & {$[0.168,0.537]$} & {$[0.086,0.897]$} & B & 84 & 0.012 & {$[0.006,0.018]$} & {$[0.003,0.032]$} & B \\
\hline 54 & 0.307 & {$[0.153,0.489]$} & {$[0.078,0.820]$} & B & 97 & 0.003 & {$[0.002,0.005]$} & {$[0.001,0.009]$} & B \\
\hline 55 & 3.676 & {$[1.733,4.757]$} & {$[0.795,6.753]$} & A & 98 & 0.128 & {$[0.063,0.203]$} & {$[0.033,0.347]$} & B \\
\hline 56 & 2.329 & {$[1.298,3.993]$} & {$[0.599,5.961]$} & B & 99 & 0.011 & {$[0.005,0.017]$} & {$[0.003,0.030]$} & B \\
\hline 57 & 0.183 & {$[0.090,0.289]$} & {$[0.047,0.488]$} & B & & & & & \\
\hline
\end{tabular}


Table 25. Partial ionization cross sections $\left(10^{-20} \mathrm{~m}^{2}\right)$ for 4-methyl-1-hexene. TICS $/ 10^{-20} \mathrm{~m}^{2}=21.365 . M=98$, base peak is $\mathrm{m} / \mathrm{z} 57$.

\begin{tabular}{|c|c|c|c|c|c|c|c|c|c|}
\hline $\mathrm{m} / \mathrm{z}$ & PICS & $67 \%$ interval & $95 \%$ interval & unc. model & $m / z$ & PICS & $67 \%$ interval & $95 \%$ interval & unc. model \\
\hline 1 & 0.400 & {$[0.288,0.478]$} & {$[0.215,0.600]$} & $\mathrm{H}$ & 57 & 3.779 & {$[1.807,4.932]$} & {$[0.845,7.011]$} & A \\
\hline 14 & 0.011 & {$[0.005,0.017]$} & {$[0.003,0.029]$} & B & 58 & 0.170 & {$[0.084,0.269]$} & {$[0.044,0.458]$} & B \\
\hline 15 & 0.125 & {$[0.061,0.195]$} & {$[0.032,0.332]$} & B & 59 & 0.004 & {$[0.002,0.006]$} & {$[0.001,0.010]$} & B \\
\hline 26 & 0.088 & {$[0.043,0.139]$} & {$[0.023,0.239]$} & B & 60 & 0.004 & {$[0.002,0.006]$} & {$[0.001,0.010]$} & B \\
\hline 27 & 0.982 & {$[0.500,1.590]$} & {$[0.251,2.588]$} & B & 61 & 0.003 & {$[0.002,0.005]$} & {$[0.001,0.009]$} & B \\
\hline 28 & 0.105 & {$[0.052,0.166]$} & {$[0.027,0.282]$} & B & 62 & 0.007 & {$[0.003,0.011]$} & {$[0.002,0.018]$} & B \\
\hline 29 & 3.628 & {$[1.655,4.751]$} & {$[0.714,6.817]$} & A & 63 & 0.010 & {$[0.005,0.016]$} & {$[0.003,0.027]$} & B \\
\hline 30 & 0.080 & {$[0.039,0.124]$} & {$[0.020,0.212]$} & B & 64 & 0.003 & {$[0.002,0.005]$} & {$[0.001,0.009]$} & B \\
\hline 31 & 0.013 & {$[0.006,0.020]$} & {$[0.003,0.035]$} & B & 65 & 0.022 & {$[0.011,0.035]$} & {$[0.006,0.060]$} & B \\
\hline 37 & 0.023 & {$[0.011,0.036]$} & {$[0.006,0.062]$} & B & 66 & 0.009 & {$[0.004,0.015]$} & {$[0.002,0.025]$} & B \\
\hline 38 & 0.072 & {$[0.035,0.114]$} & {$[0.018,0.192]$} & B & 67 & 0.088 & {$[0.043,0.137]$} & {$[0.022,0.234]$} & B \\
\hline 39 & 0.926 & {$[0.471,1.499]$} & {$[0.236,2.438]$} & B & 68 & 0.032 & {$[0.016,0.051]$} & {$[0.008,0.088]$} & B \\
\hline 40 & 0.166 & {$[0.082,0.264]$} & {$[0.043,0.447]$} & B & 69 & 0.309 & {$[0.154,0.491]$} & {$[0.079,0.822]$} & B \\
\hline 41 & 4.492 & {$[2.552,5.763]$} & {$[1.425,7.812]$} & A & 70 & 0.400 & {$[0.199,0.640]$} & {$[0.102,1.073]$} & B \\
\hline 42 & 0.437 & {$[0.218,0.696]$} & {$[0.112,1.165]$} & B & 71 & 0.025 & {$[0.012,0.039]$} & {$[0.006,0.067]$} & B \\
\hline 43 & 0.206 & {$[0.102,0.325]$} & {$[0.053,0.550]$} & B & 73 & 0.003 & {$[0.001,0.004]$} & {$[0.001,0.007]$} & B \\
\hline 44 & 0.010 & {$[0.005,0.015]$} & {$[0.003,0.027]$} & B & 77 & 0.009 & {$[0.005,0.015]$} & {$[0.002,0.025]$} & B \\
\hline 45 & 0.010 & {$[0.005,0.015]$} & {$[0.002,0.026]$} & B & 78 & 0.002 & {$[0.001,0.004]$} & {$[0.001,0.006]$} & B \\
\hline 49 & 0.004 & {$[0.002,0.007]$} & {$[0.001,0.012]$} & B & 79 & 0.009 & {$[0.004,0.014]$} & {$[0.002,0.024]$} & B \\
\hline 50 & 0.035 & {$[0.017,0.055]$} & {$[0.009,0.095]$} & B & 81 & 0.012 & {$[0.006,0.019]$} & {$[0.003,0.033]$} & B \\
\hline 51 & 0.059 & {$[0.029,0.094]$} & {$[0.015,0.161]$} & B & 82 & 0.004 & {$[0.002,0.006]$} & {$[0.001,0.011]$} & B \\
\hline 52 & 0.025 & {$[0.012,0.039]$} & {$[0.006,0.068]$} & B & 83 & 0.026 & {$[0.013,0.041]$} & {$[0.007,0.071]$} & B \\
\hline 53 & 0.176 & {$[0.087,0.278]$} & {$[0.045,0.471]$} & B & 84 & 0.002 & {$[0.001,0.003]$} & {$[0.000,0.005]$} & B \\
\hline 54 & 0.080 & {$[0.039,0.126]$} & {$[0.020,0.214]$} & B & 97 & 0.007 & {$[0.003,0.011]$} & {$[0.002,0.019]$} & B \\
\hline 55 & 0.929 & {$[0.475,1.509]$} & {$[0.239,2.455]$} & B & 98 & 0.083 & {$[0.040,0.130]$} & {$[0.021,0.223]$} & B \\
\hline 56 & 3.253 & {$[1.255,4.288]$} & {$[0.445,6.396]$} & A & 99 & 0.009 & {$[0.004,0.013]$} & {$[0.002,0.023]$} & B \\
\hline
\end{tabular}

Table 26. Partial ionization cross sections $\left(10^{-20} \mathrm{~m}^{2}\right)$ for 5 -methyl-1-hexene. TICS $/ 10^{-20} \mathrm{~m}^{2}=21.018 . M=98$, base peak is $\mathrm{m} / \mathrm{z} 56$.

\begin{tabular}{|c|c|c|c|c|c|c|c|c|c|}
\hline$m / z$ & PICS & $67 \%$ interval & 95\% interval & unc. model & $m / z$ & PICS & $67 \%$ interval & $95 \%$ interval & unc. model \\
\hline 1 & 0.400 & {$[0.288,0.478]$} & {$[0.215,0.600]$} & $\mathrm{H}$ & 57 & 1.661 & {$[0.881,2.777]$} & {$[0.421,4.307]$} & B \\
\hline 14 & 0.011 & {$[0.006,0.018]$} & {$[0.003,0.031]$} & B & 58 & 0.070 & {$[0.034,0.109]$} & {$[0.018,0.187]$} & B \\
\hline 15 & 0.120 & {$[0.059,0.188]$} & {$[0.031,0.320]$} & B & 61 & 0.004 & {$[0.002,0.006]$} & {$[0.001,0.010]$} & B \\
\hline 26 & 0.093 & {$[0.045,0.146]$} & {$[0.024,0.249]$} & B & 62 & 0.007 & {$[0.003,0.011]$} & {$[0.002,0.019]$} & B \\
\hline 27 & 1.220 & {$[0.636,2.015]$} & {$[0.312,3.206]$} & B & 63 & 0.010 & {$[0.005,0.016]$} & {$[0.003,0.028]$} & B \\
\hline 28 & 0.118 & {$[0.058,0.186]$} & {$[0.030,0.315]$} & B & 65 & 0.020 & {$[0.010,0.031]$} & {$[0.005,0.054]$} & B \\
\hline 29 & 2.077 & {$[1.136,3.526]$} & {$[0.535,5.366]$} & B & 66 & 0.010 & {$[0.005,0.015]$} & {$[0.003,0.026]$} & B \\
\hline 30 & 0.049 & {$[0.024,0.077]$} & {$[0.013,0.133]$} & B & 67 & 0.066 & {$[0.032,0.104]$} & {$[0.017,0.179]$} & B \\
\hline 37 & 0.030 & {$[0.015,0.047]$} & {$[0.008,0.081]$} & B & 68 & 0.028 & {$[0.014,0.043]$} & {$[0.007,0.075]$} & B \\
\hline 38 & 0.064 & {$[0.031,0.101]$} & {$[0.016,0.172]$} & B & 69 & 0.542 & {$[0.272,0.868]$} & {$[0.138,1.434]$} & B \\
\hline 39 & 1.092 & {$[0.563,1.785]$} & {$[0.279,2.870]$} & B & 70 & 0.547 & {$[0.274,0.876]$} & {$[0.139,1.454]$} & B \\
\hline 40 & 0.163 & {$[0.080,0.257]$} & {$[0.041,0.433]$} & B & 71 & 0.029 & {$[0.014,0.045]$} & {$[0.007,0.077]$} & B \\
\hline 41 & 3.655 & {$[1.726,4.726]$} & {$[0.802,6.716]$} & A & 74 & 0.003 & {$[0.001,0.004]$} & {$[0.001,0.007]$} & B \\
\hline 42 & 0.577 & {$[0.290,0.926]$} & {$[0.148,1.530]$} & B & 77 & 0.012 & {$[0.006,0.019]$} & {$[0.003,0.033]$} & B \\
\hline 43 & 1.411 & {$[0.739,2.333]$} & {$[0.361,3.706]$} & B & 78 & 0.002 & {$[0.001,0.004]$} & {$[0.001,0.006]$} & B \\
\hline 44 & 0.047 & {$[0.023,0.073]$} & {$[0.012,0.127]$} & B & 79 & 0.011 & {$[0.006,0.018]$} & {$[0.003,0.031]$} & B \\
\hline 49 & 0.005 & {$[0.002,0.007]$} & {$[0.001,0.013]$} & B & 81 & 0.017 & {$[0.008,0.027]$} & {$[0.004,0.046]$} & B \\
\hline 50 & 0.050 & {$[0.024,0.078]$} & {$[0.013,0.135]$} & B & 82 & 0.004 & {$[0.002,0.006]$} & {$[0.001,0.011]$} & B \\
\hline 51 & 0.076 & {$[0.037,0.119]$} & {$[0.019,0.203]$} & B & 83 & 0.125 & {$[0.062,0.198]$} & {$[0.032,0.336]$} & B \\
\hline 52 & 0.035 & {$[0.017,0.056]$} & {$[0.009,0.095]$} & B & 84 & 0.010 & {$[0.005,0.015]$} & {$[0.002,0.026]$} & B \\
\hline 53 & 0.198 & {$[0.098,0.315]$} & {$[0.051,0.532]$} & B & 97 & 0.005 & {$[0.003,0.008]$} & {$[0.001,0.015]$} & B \\
\hline 54 & 0.156 & {$[0.077,0.247]$} & {$[0.040,0.418]$} & B & 98 & 0.025 & {$[0.012,0.039]$} & {$[0.006,0.068]$} & B \\
\hline 55 & 2.112 & {$[1.156,3.583]$} & {$[0.545,5.459]$} & B & 99 & 0.002 & {$[0.001,0.003]$} & {$[0.000,0.005]$} & B \\
\hline 56 & 4.049 & {$[2.135,5.201]$} & {$[1.112,7.186]$} & A & & & & & \\
\hline
\end{tabular}


Table 27. Partial ionization cross sections $\left(10^{-20} \mathrm{~m}^{2}\right)$ for 2-methyl-2-hexene. TICS $/ 10^{-20} \mathrm{~m}^{2}=21.435 . M=98$, base peak is $m / z 69$.

\begin{tabular}{|c|c|c|c|c|c|c|c|c|c|}
\hline$m / z$ & PICS & $67 \%$ interval & $95 \%$ interval & unc. model & $m / z$ & PICS & $67 \%$ interval & $95 \%$ interval & unc. model \\
\hline 1 & 0.400 & {$[0.287,0.477]$} & {$[0.216,0.600]$} & $\mathrm{H}$ & 57 & 0.094 & {$[0.046,0.148]$} & {$[0.024,0.253]$} & B \\
\hline 12 & 0.026 & {$[0.013,0.041]$} & {$[0.007,0.070]$} & B & 58 & 0.004 & {$[0.002,0.006]$} & {$[0.001,0.011]$} & B \\
\hline 13 & 0.025 & {$[0.012,0.038]$} & {$[0.006,0.066]$} & B & 60 & 0.004 & {$[0.002,0.006]$} & {$[0.001,0.010]$} & B \\
\hline 14 & 0.094 & {$[0.046,0.148]$} & {$[0.024,0.253]$} & B & 61 & 0.008 & {$[0.004,0.012]$} & {$[0.002,0.020]$} & B \\
\hline 15 & 0.828 & {$[0.420,1.342]$} & {$[0.212,2.196]$} & B & 62 & 0.018 & {$[0.009,0.028]$} & {$[0.005,0.049]$} & B \\
\hline 16 & 0.033 & {$[0.016,0.051]$} & {$[0.008,0.088]$} & B & 63 & 0.032 & {$[0.016,0.051]$} & {$[0.008,0.088]$} & B \\
\hline 24 & 0.008 & {$[0.004,0.013]$} & {$[0.002,0.023]$} & B & 64 & 0.007 & {$[0.003,0.011]$} & {$[0.002,0.019]$} & B \\
\hline 25 & 0.016 & {$[0.008,0.026]$} & {$[0.004,0.044]$} & B & 65 & 0.048 & {$[0.023,0.075]$} & {$[0.012,0.129]$} & B \\
\hline 26 & 0.305 & {$[0.151,0.485]$} & {$[0.078,0.813]$} & B & 66 & 0.020 & {$[0.010,0.032]$} & {$[0.005,0.054]$} & B \\
\hline 27 & 2.502 & {$[1.414,4.312]$} & {$[0.646,6.373]$} & B & 67 & 0.164 & {$[0.081,0.259]$} & {$[0.042,0.439]$} & B \\
\hline 28 & 0.411 & {$[0.205,0.656]$} & {$[0.105,1.091]$} & B & 68 & 0.128 & {$[0.063,0.202]$} & {$[0.033,0.344]$} & B \\
\hline 29 & 0.989 & {$[0.509,1.615]$} & {$[0.254,2.620]$} & B & 69 & 3.122 & {$[1.132,4.068]$} & {$[0.367,6.083]$} & A \\
\hline 30 & 0.022 & {$[0.011,0.034]$} & {$[0.006,0.059]$} & B & 70 & 0.211 & {$[0.105,0.336]$} & {$[0.054,0.563]$} & B \\
\hline 31 & 0.007 & {$[0.003,0.011]$} & {$[0.002,0.019]$} & B & 71 & 0.006 & {$[0.003,0.009]$} & {$[0.002,0.016]$} & B \\
\hline 32 & 0.007 & {$[0.003,0.011]$} & {$[0.002,0.019]$} & B & 73 & 0.003 & {$[0.001,0.004]$} & {$[0.001,0.008]$} & B \\
\hline 36 & 0.006 & {$[0.003,0.010]$} & {$[0.002,0.017]$} & B & 74 & 0.005 & {$[0.003,0.008]$} & {$[0.001,0.015]$} & B \\
\hline 37 & 0.062 & {$[0.030,0.097]$} & {$[0.016,0.166]$} & B & 75 & 0.003 & {$[0.001,0.004]$} & {$[0.001,0.007]$} & B \\
\hline 38 & 0.169 & {$[0.083,0.267]$} & {$[0.043,0.455]$} & B & 76 & 0.003 & {$[0.001,0.004]$} & {$[0.001,0.007]$} & B \\
\hline 39 & 1.744 & {$[0.940,2.952]$} & {$[0.446,4.542]$} & B & 77 & 0.023 & {$[0.011,0.035]$} & {$[0.006,0.061]$} & B \\
\hline 40 & 0.284 & {$[0.142,0.454]$} & {$[0.073,0.760]$} & B & 78 & 0.005 & {$[0.002,0.008]$} & {$[0.001,0.013]$} & B \\
\hline 41 & 4.985 & {$[3.060,6.302]$} & {$[1.847,8.293]$} & A & 79 & 0.019 & {$[0.009,0.029]$} & {$[0.005,0.051]$} & B \\
\hline 42 & 0.295 & {$[0.147,0.470]$} & {$[0.076,0.791]$} & B & 80 & 0.005 & {$[0.002,0.007]$} & {$[0.001,0.012]$} & B \\
\hline 43 & 0.415 & {$[0.206,0.658]$} & {$[0.106,1.109]$} & B & 81 & 0.031 & {$[0.015,0.049]$} & {$[0.008,0.084]$} & B \\
\hline 44 & 0.016 & {$[0.008,0.025]$} & {$[0.004,0.043]$} & B & 82 & 0.011 & {$[0.005,0.017]$} & {$[0.003,0.029]$} & B \\
\hline 45 & 0.005 & {$[0.003,0.008]$} & {$[0.001,0.014]$} & B & 83 & 0.158 & {$[0.078,0.250]$} & {$[0.040,0.423]$} & B \\
\hline 46 & 0.005 & {$[0.002,0.008]$} & {$[0.001,0.014]$} & B & 84 & 0.010 & {$[0.005,0.015]$} & {$[0.003,0.026]$} & B \\
\hline 47 & 0.005 & {$[0.002,0.008]$} & {$[0.001,0.014]$} & B & 85 & 0.002 & {$[0.001,0.003]$} & {$[0.000,0.005]$} & B \\
\hline 48 & 0.005 & {$[0.002,0.008]$} & {$[0.001,0.013]$} & B & 91 & 0.002 & {$[0.001,0.003]$} & {$[0.000,0.005]$} & B \\
\hline 49 & 0.010 & {$[0.005,0.015]$} & {$[0.002,0.026]$} & B & 92 & 0.002 & {$[0.001,0.003]$} & {$[0.000,0.005]$} & B \\
\hline 50 & 0.118 & {$[0.058,0.184]$} & {$[0.030,0.315]$} & B & 93 & 0.002 & {$[0.001,0.003]$} & {$[0.000,0.005]$} & B \\
\hline 51 & 0.199 & {$[0.099,0.316]$} & {$[0.051,0.530]$} & B & 95 & 0.002 & {$[0.001,0.003]$} & {$[0.000,0.005]$} & B \\
\hline 52 & 0.082 & {$[0.040,0.129]$} & {$[0.021,0.221]$} & B & 96 & 0.002 & {$[0.001,0.003]$} & {$[0.000,0.005]$} & B \\
\hline 53 & 0.458 & {$[0.229,0.730]$} & {$[0.117,1.213]$} & B & 97 & 0.004 & {$[0.002,0.006]$} & {$[0.001,0.010]$} & B \\
\hline 54 & 0.122 & {$[0.060,0.193]$} & {$[0.031,0.326]$} & B & 98 & 0.466 & {$[0.234,0.748]$} & {$[0.119,1.234]$} & B \\
\hline 55 & 1.027 & {$[0.524,1.664]$} & {$[0.263,2.707]$} & B & 99 & 0.035 & {$[0.017,0.055]$} & {$[0.009,0.096]$} & B \\
\hline 56 & 1.094 & {$[0.566,1.802]$} & {$[0.279,2.881]$} & B & 100 & 0.002 & {$[0.001,0.003]$} & {$[0.000,0.005]$} & B \\
\hline
\end{tabular}


Table 28. Partial ionization cross sections $\left(10^{-20} \mathrm{~m}^{2}\right)$ for 3-methyl-2-hexene. TICS $/ 10^{-20} \mathrm{~m}^{2}=21.270 . M=98$, base peak is $\mathrm{m} / \mathrm{z} 41$.

\begin{tabular}{|c|c|c|c|c|c|c|c|c|c|}
\hline$m / z$ & PICS & $67 \%$ interval & $95 \%$ interval & unc. model & $m / z$ & PICS & $67 \%$ interval & $95 \%$ interval & unc. model \\
\hline 1 & 0.400 & {$[0.287,0.477]$} & {$[0.216,0.600]$} & $\mathrm{H}$ & 61 & 0.016 & {$[0.008,0.025]$} & {$[0.004,0.044]$} & B \\
\hline 25 & 0.060 & {$[0.030,0.095]$} & {$[0.016,0.163]$} & B & 62 & 0.016 & {$[0.008,0.025]$} & {$[0.004,0.043]$} & B \\
\hline 26 & 0.290 & {$[0.143,0.459]$} & {$[0.074,0.773]$} & B & 63 & 0.033 & {$[0.016,0.053]$} & {$[0.009,0.090]$} & B \\
\hline 27 & 1.635 & {$[0.873,2.746]$} & {$[0.418,4.260]$} & B & 65 & 0.068 & {$[0.033,0.108]$} & {$[0.018,0.185]$} & B \\
\hline 28 & 0.495 & {$[0.247,0.789]$} & {$[0.125,1.306]$} & B & 66 & 0.031 & {$[0.015,0.049]$} & {$[0.008,0.084]$} & B \\
\hline 29 & 1.212 & {$[0.627,1.989]$} & {$[0.310,3.190]$} & B & 67 & 0.225 & {$[0.111,0.357]$} & {$[0.057,0.599]$} & B \\
\hline 30 & 0.044 & {$[0.021,0.068]$} & {$[0.011,0.117]$} & B & 68 & 0.061 & {$[0.030,0.096]$} & {$[0.016,0.165]$} & B \\
\hline 31 & 0.045 & {$[0.022,0.071]$} & {$[0.012,0.122]$} & B & 69 & 2.525 & {$[0.472,3.194]$} & {$[0.050,5.304]$} & A \\
\hline 33 & 0.021 & {$[0.010,0.033]$} & {$[0.005,0.057]$} & B & 70 & 0.773 & {$[0.392,1.246]$} & {$[0.197,2.034]$} & B \\
\hline 34 & 0.015 & {$[0.007,0.024]$} & {$[0.004,0.041]$} & B & 71 & 0.043 & {$[0.021,0.067]$} & {$[0.011,0.114]$} & B \\
\hline 36 & 0.050 & {$[0.025,0.079]$} & {$[0.013,0.136]$} & B & 73 & 0.011 & {$[0.005,0.017]$} & {$[0.003,0.030]$} & B \\
\hline 37 & 0.055 & {$[0.027,0.086]$} & {$[0.014,0.147]$} & B & 74 & 0.006 & {$[0.003,0.010]$} & {$[0.002,0.017]$} & B \\
\hline 38 & 0.181 & {$[0.089,0.285]$} & {$[0.046,0.483]$} & B & 75 & 0.008 & {$[0.004,0.013]$} & {$[0.002,0.022]$} & B \\
\hline 39 & 1.443 & {$[0.762,2.412]$} & {$[0.368,3.793]$} & B & 76 & 0.009 & {$[0.005,0.015]$} & {$[0.002,0.025]$} & B \\
\hline 40 & 0.221 & {$[0.110,0.351]$} & {$[0.057,0.590]$} & B & 77 & 0.025 & {$[0.012,0.040]$} & {$[0.007,0.069]$} & B \\
\hline 41 & 5.689 & {$[3.788,7.107]$} & {$[2.483,9.108]$} & A & 78 & 0.022 & {$[0.011,0.035]$} & {$[0.006,0.060]$} & B \\
\hline 42 & 0.467 & {$[0.235,0.749]$} & {$[0.120,1.242]$} & B & 79 & 0.025 & {$[0.012,0.038]$} & {$[0.006,0.066]$} & B \\
\hline 43 & 0.397 & {$[0.199,0.638]$} & {$[0.102,1.062]$} & B & 80 & 0.008 & {$[0.004,0.013]$} & {$[0.002,0.022]$} & B \\
\hline 44 & 0.041 & {$[0.020,0.064]$} & {$[0.010,0.110]$} & B & 81 & 0.024 & {$[0.012,0.038]$} & {$[0.006,0.065]$} & B \\
\hline 45 & 0.020 & {$[0.010,0.032]$} & {$[0.005,0.055]$} & B & 82 & 0.020 & {$[0.010,0.031]$} & {$[0.005,0.053]$} & B \\
\hline 46 & 0.022 & {$[0.010,0.034]$} & {$[0.006,0.058]$} & B & 83 & 0.224 & {$[0.110,0.353]$} & {$[0.057,0.596]$} & B \\
\hline 47 & 0.011 & {$[0.005,0.017]$} & {$[0.003,0.030]$} & B & 84 & 0.020 & {$[0.010,0.031]$} & {$[0.005,0.053]$} & B \\
\hline 48 & 0.022 & {$[0.011,0.034]$} & {$[0.006,0.059]$} & B & 85 & 0.003 & {$[0.001,0.004]$} & {$[0.001,0.008]$} & B \\
\hline 50 & 0.102 & {$[0.050,0.162]$} & {$[0.026,0.274]$} & B & 86 & 0.007 & {$[0.003,0.010]$} & {$[0.002,0.018]$} & B \\
\hline 51 & 0.159 & {$[0.079,0.254]$} & {$[0.041,0.429]$} & B & 88 & 0.005 & {$[0.002,0.007]$} & {$[0.001,0.013]$} & B \\
\hline 52 & 0.077 & {$[0.038,0.122]$} & {$[0.020,0.207]$} & B & 90 & 0.005 & {$[0.002,0.008]$} & {$[0.001,0.014]$} & B \\
\hline 53 & 0.320 & {$[0.159,0.510]$} & {$[0.082,0.850]$} & B & 91 & 0.011 & {$[0.005,0.017]$} & {$[0.003,0.029]$} & B \\
\hline 54 & 0.087 & {$[0.043,0.138]$} & {$[0.023,0.236]$} & B & 92 & 0.009 & {$[0.004,0.013]$} & {$[0.002,0.023]$} & B \\
\hline 55 & 2.245 & {$[1.258,3.877]$} & {$[0.580,5.789]$} & B & 93 & 0.008 & {$[0.004,0.012]$} & {$[0.002,0.022]$} & B \\
\hline 56 & 0.836 & {$[0.426,1.356]$} & {$[0.212,2.200]$} & B & 94 & 0.004 & {$[0.002,0.006]$} & {$[0.001,0.011]$} & B \\
\hline 57 & 0.108 & {$[0.053,0.170]$} & {$[0.028,0.292]$} & B & 95 & 0.004 & {$[0.002,0.006]$} & {$[0.001,0.011]$} & B \\
\hline 58 & 0.012 & {$[0.006,0.019]$} & {$[0.003,0.034]$} & B & 96 & 0.003 & {$[0.002,0.005]$} & {$[0.001,0.009]$} & B \\
\hline 59 & 0.007 & {$[0.003,0.010]$} & {$[0.002,0.018]$} & B & 98 & 0.203 & {$[0.100,0.322]$} & {$[0.052,0.548]$} & B \\
\hline 60 & 0.007 & {$[0.004,0.011]$} & {$[0.002,0.020]$} & B & 99 & 0.018 & {$[0.009,0.029]$} & {$[0.005,0.050]$} & B \\
\hline
\end{tabular}

Table 29. Partial ionization cross sections $\left(10^{-20} \mathrm{~m}^{2}\right)$ for 4-methyl-2-hexene. TICS/10 $0^{-20} \mathrm{~m}^{2}=21.403 . M=98$, base peak is $m / z 69$.

\begin{tabular}{|c|c|c|c|c|c|c|c|c|c|}
\hline$m / z$ & PICS & $67 \%$ interval & $95 \%$ interval & unc. model & $m / z$ & PICS & $67 \%$ interval & $95 \%$ interval & unc. model \\
\hline 1 & 0.400 & {$[0.288,0.478]$} & {$[0.215,0.600]$} & $\mathrm{H}$ & 53 & 0.455 & {$[0.228,0.729]$} & {$[0.116,1.209]$} & B \\
\hline 14 & 0.200 & {$[0.099,0.317]$} & {$[0.051,0.534]$} & B & 55 & 1.863 & {$[1.010,3.162]$} & {$[0.478,4.851]$} & B \\
\hline 15 & 0.370 & {$[0.186,0.594]$} & {$[0.095,0.991]$} & B & 56 & 0.571 & {$[0.288,0.918]$} & {$[0.145,1.509]$} & B \\
\hline 26 & 0.227 & {$[0.113,0.364]$} & {$[0.058,0.604]$} & B & 57 & 0.075 & {$[0.037,0.118]$} & {$[0.019,0.202]$} & B \\
\hline 27 & 1.550 & {$[0.823,2.598]$} & {$[0.395,4.057]$} & B & 63 & 0.057 & {$[0.028,0.091]$} & {$[0.015,0.156]$} & B \\
\hline 28 & 1.081 & {$[0.557,1.767]$} & {$[0.278,2.863]$} & B & 65 & 0.104 & {$[0.051,0.163]$} & {$[0.027,0.278]$} & B \\
\hline 29 & 0.966 & {$[0.495,1.576]$} & {$[0.246,2.540]$} & B & 66 & 0.048 & {$[0.023,0.075]$} & {$[0.012,0.130]$} & B \\
\hline 32 & 0.227 & {$[0.112,0.360]$} & {$[0.058,0.609]$} & B & 67 & 0.338 & {$[0.169,0.539]$} & {$[0.087,0.904]$} & B \\
\hline 38 & 0.185 & {$[0.092,0.296]$} & {$[0.048,0.498]$} & B & 69 & 3.677 & {$[1.734,4.770]$} & {$[0.774,6.738]$} & A \\
\hline 39 & 1.792 & {$[0.965,3.029]$} & {$[0.458,4.663]$} & B & 70 & 0.495 & {$[0.247,0.789]$} & {$[0.126,1.304]$} & B \\
\hline 41 & 4.949 & {$[3.010,6.235]$} & {$[1.839,8.251]$} & A & 77 & 0.035 & {$[0.017,0.054]$} & {$[0.009,0.093]$} & B \\
\hline 42 & 0.312 & {$[0.155,0.496]$} & {$[0.080,0.833]$} & B & 79 & 0.034 & {$[0.017,0.053]$} & {$[0.009,0.092]$} & B \\
\hline 43 & 0.194 & {$[0.096,0.308]$} & {$[0.050,0.519]$} & B & 83 & 0.258 & {$[0.127,0.408]$} & {$[0.066,0.692]$} & B \\
\hline 50 & 0.108 & {$[0.053,0.170]$} & {$[0.028,0.289]$} & B & 98 & 0.497 & {$[0.249,0.795]$} & {$[0.127,1.323]$} & B \\
\hline 51 & 0.199 & {$[0.098,0.314]$} & {$[0.051,0.536]$} & B & 99 & 0.043 & {$[0.021,0.068]$} & {$[0.011,0.116]$} & B \\
\hline 52 & 0.090 & {$[0.044,0.142]$} & {$[0.023,0.243]$} & B & & & & & \\
\hline
\end{tabular}


Table 30. Partial ionization cross sections $\left(10^{-20} \mathrm{~m}^{2}\right)$ for 5-methyl-2-hexene. TICS $/ 10^{-20} \mathrm{~m}^{2}=21.309 . M=98$, base peak is $\mathrm{m} / \mathrm{z} 56$.

\begin{tabular}{|c|c|c|c|c|c|c|c|c|c|}
\hline$m / z$ & PICS & $67 \%$ interval & $95 \%$ interval & unc. model & $m / \mathbf{z}$ & PICS & $67 \%$ interval & $95 \%$ interval & unc. model \\
\hline 1 & 0.400 & {$[0.288,0.478]$} & {$[0.215,0.599]$} & $\mathrm{H}$ & 59 & 0.003 & {$[0.001,0.004]$} & {$[0.001,0.008]$} & B \\
\hline 12 & 0.009 & {$[0.004,0.014]$} & {$[0.002,0.025]$} & B & 60 & 0.003 & {$[0.001,0.004]$} & {$[0.001,0.007]$} & B \\
\hline 13 & 0.026 & {$[0.013,0.041]$} & {$[0.007,0.071]$} & B & 61 & 0.005 & {$[0.003,0.008]$} & {$[0.001,0.014]$} & B \\
\hline 14 & 0.075 & {$[0.037,0.118]$} & {$[0.019,0.202]$} & B & 62 & 0.010 & {$[0.005,0.016]$} & {$[0.003,0.028]$} & B \\
\hline 15 & 0.684 & {$[0.346,1.102]$} & {$[0.174,1.809]$} & B & 63 & 0.023 & {$[0.011,0.036]$} & {$[0.006,0.061]$} & B \\
\hline 16 & 0.031 & {$[0.015,0.048]$} & {$[0.008,0.083]$} & B & 64 & 0.002 & {$[0.001,0.004]$} & {$[0.001,0.007]$} & B \\
\hline 19 & 0.007 & {$[0.003,0.011]$} & {$[0.002,0.019]$} & B & 65 & 0.034 & {$[0.017,0.053]$} & {$[0.009,0.092]$} & B \\
\hline 20 & 0.007 & {$[0.003,0.010]$} & {$[0.002,0.018]$} & B & 66 & 0.017 & {$[0.008,0.026]$} & {$[0.004,0.045]$} & B \\
\hline 24 & 0.006 & {$[0.003,0.009]$} & {$[0.002,0.016]$} & B & 67 & 0.093 & {$[0.046,0.146]$} & {$[0.024,0.250]$} & B \\
\hline 25 & 0.006 & {$[0.003,0.009]$} & {$[0.002,0.016]$} & B & 68 & 0.029 & {$[0.014,0.046]$} & {$[0.008,0.080]$} & B \\
\hline 26 & 0.205 & {$[0.102,0.326]$} & {$[0.053,0.550]$} & B & 69 & 0.432 & {$[0.217,0.690]$} & {$[0.111,1.144]$} & B \\
\hline 27 & 2.400 & {$[1.329,4.102]$} & {$[0.616,6.158]$} & B & 70 & 0.309 & {$[0.153,0.489]$} & {$[0.079,0.826]$} & B \\
\hline 28 & 0.400 & {$[0.198,0.633]$} & {$[0.102,1.063]$} & B & 71 & 0.017 & {$[0.008,0.026]$} & {$[0.004,0.046]$} & B \\
\hline 29 & 1.186 & {$[0.616,1.951]$} & {$[0.302,3.099]$} & B & 72 & 0.002 & {$[0.001,0.003]$} & {$[0.001,0.005]$} & B \\
\hline 30 & 0.026 & {$[0.013,0.040]$} & {$[0.007,0.070]$} & B & 73 & 0.002 & {$[0.001,0.003]$} & {$[0.001,0.005]$} & B \\
\hline 31 & 0.005 & {$[0.002,0.008]$} & {$[0.001,0.014]$} & B & 74 & 0.004 & {$[0.002,0.006]$} & {$[0.001,0.011]$} & B \\
\hline 36 & 0.004 & {$[0.002,0.007]$} & {$[0.001,0.012]$} & B & 75 & 0.002 & {$[0.001,0.003]$} & {$[0.000,0.005]$} & B \\
\hline 37 & 0.039 & {$[0.019,0.062]$} & {$[0.010,0.106]$} & B & 76 & 0.002 & {$[0.001,0.003]$} & {$[0.000,0.005]$} & B \\
\hline 38 & 0.120 & {$[0.059,0.191]$} & {$[0.031,0.324]$} & B & 77 & 0.021 & {$[0.010,0.033]$} & {$[0.005,0.057]$} & B \\
\hline 39 & 1.379 & {$[0.724,2.284]$} & {$[0.352,3.593]$} & B & 78 & 0.003 & {$[0.002,0.005]$} & {$[0.001,0.009]$} & B \\
\hline 40 & 0.210 & {$[0.104,0.333]$} & {$[0.054,0.561]$} & B & 79 & 0.015 & {$[0.007,0.023]$} & {$[0.004,0.041]$} & B \\
\hline 41 & 2.507 & {$[1.403,4.315]$} & {$[0.645,6.423]$} & B & 80 & 0.002 & {$[0.001,0.003]$} & {$[0.000,0.004]$} & B \\
\hline 42 & 0.280 & {$[0.139,0.443]$} & {$[0.072,0.752]$} & B & 81 & 0.023 & {$[0.011,0.037]$} & {$[0.006,0.063]$} & B \\
\hline 43 & 3.050 & {$[1.047,3.971]$} & {$[0.335,6.064]$} & A & 82 & 0.005 & {$[0.002,0.007]$} & {$[0.001,0.012]$} & B \\
\hline 44 & 0.098 & {$[0.049,0.155]$} & {$[0.025,0.263]$} & B & 83 & 0.097 & {$[0.048,0.153]$} & {$[0.025,0.261]$} & B \\
\hline 45 & 0.004 & {$[0.002,0.006]$} & {$[0.001,0.010]$} & B & 84 & 0.006 & {$[0.003,0.009]$} & {$[0.001,0.015]$} & B \\
\hline 46 & 0.004 & {$[0.002,0.006]$} & {$[0.001,0.010]$} & B & 85 & 0.001 & {$[0.001,0.002]$} & {$[0.000,0.004]$} & B \\
\hline 47 & 0.004 & {$[0.002,0.006]$} & {$[0.001,0.010]$} & B & 86 & 0.001 & {$[0.001,0.002]$} & {$[0.000,0.004]$} & B \\
\hline 48 & 0.003 & {$[0.002,0.005]$} & {$[0.001,0.009]$} & B & 87 & 0.001 & {$[0.001,0.002]$} & {$[0.000,0.004]$} & B \\
\hline 49 & 0.007 & {$[0.003,0.011]$} & {$[0.002,0.018]$} & B & 89 & 0.001 & {$[0.001,0.002]$} & {$[0.000,0.004]$} & B \\
\hline 50 & 0.104 & {$[0.051,0.164]$} & {$[0.027,0.278]$} & B & 91 & 0.001 & {$[0.001,0.002]$} & {$[0.000,0.004]$} & B \\
\hline 51 & 0.161 & {$[0.080,0.256]$} & {$[0.041,0.431]$} & B & 92 & 0.001 & {$[0.001,0.002]$} & {$[0.000,0.004]$} & B \\
\hline 52 & 0.067 & {$[0.033,0.106]$} & {$[0.017,0.181]$} & B & 93 & 0.001 & {$[0.001,0.002]$} & {$[0.000,0.004]$} & B \\
\hline 53 & 0.287 & {$[0.144,0.460]$} & {$[0.074,0.772]$} & B & 95 & 0.001 & {$[0.001,0.002]$} & {$[0.000,0.004]$} & B \\
\hline 54 & 0.416 & {$[0.207,0.663]$} & {$[0.107,1.106]$} & B & 96 & 0.001 & {$[0.001,0.002]$} & {$[0.000,0.004]$} & B \\
\hline 55 & 2.481 & {$[1.383,4.248]$} & {$[0.640,6.347]$} & B & 97 & 0.011 & {$[0.005,0.016]$} & {$[0.003,0.028]$} & B \\
\hline 56 & 2.957 & {$[0.935,3.838]$} & {$[0.269,5.950]$} & A & 98 & 0.186 & {$[0.092,0.295]$} & {$[0.048,0.498]$} & B \\
\hline 57 & 0.258 & {$[0.128,0.410]$} & {$[0.066,0.691]$} & B & 99 & 0.015 & {$[0.007,0.023]$} & {$[0.004,0.039]$} & B \\
\hline 58 & 0.009 & {$[0.004,0.013]$} & {$[0.002,0.023]$} & B & 100 & 0.001 & {$[0.001,0.002]$} & {$[0.000,0.004]$} & B \\
\hline
\end{tabular}

Table 31. Partial ionization cross sections $\left(10^{-20} \mathrm{~m}^{2}\right)$ for 2-methyl-3-hexene. TICS $/ 10^{-20} \mathrm{~m}^{2}=21.250 . M=98$, base peak is $\mathrm{m} / \mathrm{z} 69$.

\begin{tabular}{|c|c|c|c|c|c|c|c|c|c|}
\hline$m / z$ & PICS & $67 \%$ interval & $95 \%$ interval & unc. model & $\mathrm{m} / \mathrm{z}$ & PICS & $67 \%$ interval & $95 \%$ interval & unc. model \\
\hline 1 & 0.400 & {$[0.288,0.478]$} & {$[0.215,0.599]$} & $\mathrm{H}$ & 53 & 0.485 & {$[0.242,0.773]$} & {$[0.124,1.288]$} & B \\
\hline 14 & 0.550 & {$[0.275,0.879]$} & {$[0.140,1.453]$} & B & 55 & 2.728 & {$[0.688,3.579]$} & {$[0.123,5.696]$} & A \\
\hline 15 & 0.833 & {$[0.425,1.352]$} & {$[0.212,2.192]$} & B & 56 & 1.214 & {$[0.628,1.984]$} & {$[0.311,3.187]$} & B \\
\hline 16 & 0.190 & {$[0.094,0.301]$} & {$[0.049,0.510]$} & B & 57 & 0.098 & {$[0.048,0.154]$} & {$[0.025,0.263]$} & B \\
\hline 26 & 0.233 & {$[0.116,0.371]$} & {$[0.060,0.623]$} & B & 63 & 0.060 & {$[0.029,0.094]$} & {$[0.015,0.160]$} & B \\
\hline 27 & 0.586 & {$[0.294,0.938]$} & {$[0.149,1.551]$} & B & 65 & 0.137 & {$[0.067,0.215]$} & {$[0.035,0.368]$} & B \\
\hline 28 & 1.402 & {$[0.737,2.326]$} & {$[0.359,3.671]$} & B & 66 & 0.061 & {$[0.030,0.095]$} & {$[0.016,0.164]$} & B \\
\hline 29 & 0.827 & {$[0.421,1.341]$} & {$[0.211,2.178]$} & B & 67 & 0.358 & {$[0.178,0.569]$} & {$[0.092,0.954]$} & B \\
\hline 38 & 0.203 & {$[0.099,0.319]$} & {$[0.052,0.540]$} & B & 69 & 2.270 & {$[1.256,3.876]$} & {$[0.580,5.780]$} & B \\
\hline 39 & 2.104 & {$[1.146,3.560]$} & {$[0.539,5.416]$} & B & 70 & 0.228 & {$[0.113,0.361]$} & {$[0.058,0.607]$} & B \\
\hline 40 & 0.384 & {$[0.191,0.611]$} & {$[0.098,1.019]$} & B & 77 & 0.066 & {$[0.032,0.103]$} & {$[0.017,0.177]$} & B \\
\hline 41 & 3.609 & {$[1.650,4.751]$} & {$[0.703,6.766]$} & A & 79 & 0.050 & {$[0.024,0.077]$} & {$[0.013,0.134]$} & B \\
\hline 42 & 0.291 & {$[0.144,0.463]$} & {$[0.074,0.776]$} & B & 81 & 0.040 & {$[0.020,0.063]$} & {$[0.010,0.108]$} & B \\
\hline 43 & 0.571 & {$[0.287,0.915]$} & {$[0.146,1.512]$} & B & 83 & 0.243 & {$[0.119,0.382]$} & {$[0.062,0.649]$} & B \\
\hline 50 & 0.175 & {$[0.086,0.277]$} & {$[0.045,0.469]$} & B & 98 & 0.416 & {$[0.208,0.666]$} & {$[0.106,1.108]$} & B \\
\hline 51 & 0.289 & {$[0.144,0.459]$} & {$[0.074,0.770]$} & B & 99 & 0.035 & {$[0.017,0.055]$} & {$[0.009,0.095]$} & B \\
\hline
\end{tabular}


Table 32. Partial ionization cross sections $\left(10^{-20} \mathrm{~m}^{2}\right)$ for 3-methyl-3-hexene. TICS $/ 10^{-20} \mathrm{~m}^{2}=21.467 . M=98$, base peak is $\mathrm{m} / \mathrm{z} 69$.

\begin{tabular}{|c|c|c|c|c|c|c|c|c|c|}
\hline$m / z$ & PICS & $67 \%$ interval & $95 \%$ interval & unc. model & $m / z$ & PICS & $67 \%$ interval & $95 \%$ interval & unc. model \\
\hline 1 & 0.400 & {$[0.288,0.478]$} & {$[0.216,0.600]$} & $\mathrm{H}$ & 57 & 0.051 & {$[0.025,0.081]$} & {$[0.013,0.139]$} & B \\
\hline 12 & 0.022 & {$[0.010,0.034]$} & {$[0.006,0.058]$} & B & 58 & 0.003 & {$[0.002,0.005]$} & {$[0.001,0.009]$} & B \\
\hline 13 & 0.031 & {$[0.015,0.048]$} & {$[0.008,0.083]$} & B & 60 & 0.003 & {$[0.002,0.005]$} & {$[0.001,0.009]$} & B \\
\hline 14 & 0.138 & {$[0.068,0.217]$} & {$[0.035,0.370]$} & B & 61 & 0.013 & {$[0.006,0.019]$} & {$[0.003,0.034]$} & B \\
\hline 15 & 1.111 & {$[0.577,1.826]$} & {$[0.284,2.909]$} & B & 62 & 0.025 & {$[0.012,0.039]$} & {$[0.006,0.067]$} & B \\
\hline 16 & 0.037 & {$[0.018,0.057]$} & {$[0.009,0.098]$} & B & 63 & 0.045 & {$[0.022,0.071]$} & {$[0.012,0.122]$} & B \\
\hline 24 & 0.007 & {$[0.003,0.011]$} & {$[0.002,0.019]$} & B & 64 & 0.009 & {$[0.004,0.014]$} & {$[0.002,0.024]$} & B \\
\hline 25 & 0.014 & {$[0.007,0.022]$} & {$[0.004,0.037]$} & B & 65 & 0.069 & {$[0.034,0.109]$} & {$[0.018,0.186]$} & B \\
\hline 26 & 0.255 & {$[0.125,0.402]$} & {$[0.065,0.681]$} & B & 66 & 0.031 & {$[0.015,0.048]$} & {$[0.008,0.083]$} & B \\
\hline 27 & 2.298 & {$[1.269,3.918]$} & {$[0.589,5.880]$} & B & 67 & 0.223 & {$[0.111,0.355]$} & {$[0.057,0.595]$} & B \\
\hline 28 & 0.428 & {$[0.214,0.686]$} & {$[0.109,1.141]$} & B & 68 & 0.088 & {$[0.043,0.138]$} & {$[0.022,0.236]$} & B \\
\hline 29 & 1.078 & {$[0.556,1.763]$} & {$[0.277,2.843]$} & B & 69 & 2.615 & {$[0.544,3.329]$} & {$[0.078,5.485]$} & A \\
\hline 30 & 0.024 & {$[0.012,0.038]$} & {$[0.006,0.065]$} & B & 70 & 0.306 & {$[0.152,0.487]$} & {$[0.078,0.815]$} & B \\
\hline 31 & 0.006 & {$[0.003,0.009]$} & {$[0.002,0.016]$} & B & 71 & 0.010 & {$[0.005,0.016]$} & {$[0.003,0.027]$} & B \\
\hline 32 & 0.012 & {$[0.006,0.018]$} & {$[0.003,0.031]$} & B & 73 & 0.002 & {$[0.001,0.004]$} & {$[0.001,0.006]$} & B \\
\hline 36 & 0.005 & {$[0.003,0.008]$} & {$[0.001,0.014]$} & B & 74 & 0.005 & {$[0.002,0.007]$} & {$[0.001,0.012]$} & B \\
\hline 37 & 0.062 & {$[0.030,0.098]$} & {$[0.016,0.167]$} & B & 75 & 0.002 & {$[0.001,0.003]$} & {$[0.001,0.006]$} & B \\
\hline 38 & 0.182 & {$[0.090,0.288]$} & {$[0.047,0.487]$} & B & 76 & 0.002 & {$[0.001,0.003]$} & {$[0.001,0.006]$} & B \\
\hline 39 & 1.787 & {$[0.962,3.022]$} & {$[0.457,4.656]$} & B & 77 & 0.027 & {$[0.013,0.043]$} & {$[0.007,0.074]$} & B \\
\hline 40 & 0.292 & {$[0.146,0.467]$} & {$[0.075,0.779]$} & B & 78 & 0.006 & {$[0.003,0.010]$} & {$[0.002,0.017]$} & B \\
\hline 41 & 4.241 & {$[2.292,5.455]$} & {$[1.225,7.477]$} & A & 79 & 0.026 & {$[0.012,0.040]$} & {$[0.007,0.069]$} & B \\
\hline 42 & 0.322 & {$[0.160,0.511]$} & {$[0.082,0.853]$} & B & 80 & 0.004 & {$[0.002,0.006]$} & {$[0.001,0.010]$} & B \\
\hline 43 & 0.356 & {$[0.177,0.567]$} & {$[0.091,0.948]$} & B & 81 & 0.037 & {$[0.018,0.058]$} & {$[0.010,0.100]$} & B \\
\hline 44 & 0.013 & {$[0.006,0.021]$} & {$[0.003,0.036]$} & B & 82 & 0.011 & {$[0.005,0.017]$} & {$[0.003,0.029]$} & B \\
\hline 45 & 0.004 & {$[0.002,0.007]$} & {$[0.001,0.012]$} & B & 83 & 0.244 & {$[0.120,0.385]$} & {$[0.062,0.649]$} & B \\
\hline 46 & 0.004 & {$[0.002,0.007]$} & {$[0.001,0.012]$} & B & 84 & 0.015 & {$[0.007,0.023]$} & {$[0.004,0.040]$} & B \\
\hline 47 & 0.004 & {$[0.002,0.006]$} & {$[0.001,0.011]$} & B & 85 & 0.002 & {$[0.001,0.002]$} & {$[0.000,0.004]$} & B \\
\hline 48 & 0.004 & {$[0.002,0.006]$} & {$[0.001,0.011]$} & B & 91 & 0.002 & {$[0.001,0.002]$} & {$[0.000,0.004]$} & B \\
\hline 49 & 0.012 & {$[0.006,0.019]$} & {$[0.003,0.033]$} & B & 93 & 0.002 & {$[0.001,0.002]$} & {$[0.000,0.004]$} & B \\
\hline 50 & 0.126 & {$[0.062,0.199]$} & {$[0.033,0.341]$} & B & 95 & 0.002 & {$[0.001,0.002]$} & {$[0.000,0.004]$} & B \\
\hline 51 & 0.225 & {$[0.112,0.359]$} & {$[0.058,0.604]$} & B & 96 & 0.002 & {$[0.001,0.002]$} & {$[0.000,0.004]$} & B \\
\hline 52 & 0.087 & {$[0.043,0.138]$} & {$[0.023,0.236]$} & B & 97 & 0.003 & {$[0.002,0.005]$} & {$[0.001,0.008]$} & B \\
\hline 53 & 0.435 & {$[0.218,0.698]$} & {$[0.111,1.153]$} & B & 98 & 0.453 & {$[0.226,0.721]$} & {$[0.116,1.204]$} & B \\
\hline 54 & 0.106 & {$[0.052,0.166]$} & {$[0.027,0.282]$} & B & 99 & 0.034 & {$[0.017,0.054]$} & {$[0.009,0.093]$} & B \\
\hline 55 & 2.310 & {$[1.282,3.961]$} & {$[0.597,5.972]$} & B & 100 & 0.002 & {$[0.001,0.002]$} & {$[0.000,0.004]$} & B \\
\hline 56 & 0.657 & {$[0.330,1.053]$} & {$[0.168,1.737]$} & B & & & & & \\
\hline
\end{tabular}

Table 33. Partial ionization cross sections $\left(10^{-20} \mathrm{~m}^{2}\right)$ for 2,3-dimethyl-1-pentene. TICS $/ 10^{-20} \mathrm{~m}^{2}=21.425 . M=98$, base peak is $\mathrm{m} / \mathrm{z}$ 41.

\begin{tabular}{|c|c|c|c|c|c|c|c|c|c|}
\hline$m / z$ & PICS & $67 \%$ interval & $95 \%$ interval & unc. model & $m / z$ & PICS & $67 \%$ interval & $95 \%$ interval & unc. model \\
\hline 18 & 0.052 & {$[0.026,0.082]$} & {$[0.013,0.140]$} & B & 55 & 2.146 & {$[1.184,3.652]$} & {$[0.550,5.479]$} & B \\
\hline 27 & 1.822 & {$[0.978,3.054]$} & {$[0.467,4.724]$} & B & 57 & 0.073 & {$[0.036,0.114]$} & {$[0.019,0.196]$} & B \\
\hline 28 & 0.107 & {$[0.053,0.170]$} & {$[0.028,0.290]$} & B & 62 & 0.024 & {$[0.012,0.038]$} & {$[0.006,0.065]$} & B \\
\hline 29 & 1.114 & {$[0.578,1.828]$} & {$[0.285,2.920]$} & B & 63 & 0.037 & {$[0.018,0.058]$} & {$[0.009,0.099]$} & B \\
\hline 37 & 0.070 & {$[0.034,0.110]$} & {$[0.018,0.187]$} & B & 67 & 0.295 & {$[0.147,0.469]$} & {$[0.075,0.785]$} & B \\
\hline 38 & 0.196 & {$[0.098,0.313]$} & {$[0.050,0.527]$} & B & 69 & 2.500 & {$[1.413,4.299]$} & {$[0.638,6.280]$} & B \\
\hline 39 & 2.110 & {$[1.160,3.590]$} & {$[0.541,5.411]$} & B & 70 & 0.925 & {$[0.473,1.507]$} & {$[0.236,2.440]$} & B \\
\hline 40 & 0.424 & {$[0.211,0.675]$} & {$[0.108,1.123]$} & B & 71 & 0.049 & {$[0.024,0.076]$} & {$[0.012,0.131]$} & B \\
\hline 41 & 5.307 & {$[3.359,6.594]$} & {$[2.119,8.568]$} & A & 77 & 0.027 & {$[0.013,0.043]$} & {$[0.007,0.073]$} & B \\
\hline 42 & 0.419 & {$[0.209,0.669]$} & {$[0.106,1.106]$} & B & 79 & 0.027 & {$[0.013,0.042]$} & {$[0.007,0.073]$} & B \\
\hline 51 & 0.213 & {$[0.106,0.340]$} & {$[0.055,0.569]$} & B & 98 & 0.213 & {$[0.106,0.338]$} & {$[0.054,0.568]$} & B \\
\hline 52 & 0.086 & {$[0.042,0.134]$} & {$[0.022,0.230]$} & B & 99 & 0.018 & {$[0.009,0.028]$} & {$[0.005,0.048]$} & B \\
\hline
\end{tabular}


Table 34. Partial ionization cross sections $\left(10^{-20} \mathrm{~m}^{2}\right)$ for 2,4-dimethyl-1-pentene. TICS $/ 10^{-20} \mathrm{~m}^{2}=21.589 . M=98$, base peak is $\mathrm{m} / \mathrm{z}$ 56.

\begin{tabular}{|c|c|c|c|c|c|c|c|c|c|}
\hline$m / z$ & PICS & $67 \%$ interval & $95 \%$ interval & unc. model & $m / z$ & PICS & $67 \%$ interval & $95 \%$ interval & unc. model \\
\hline 1 & 0.400 & {$[0.288,0.478]$} & {$[0.215,0.600]$} & $\mathrm{H}$ & 55 & 1.331 & {$[0.694,2.199]$} & {$[0.338,3.478]$} & B \\
\hline 15 & 0.528 & {$[0.264,0.844]$} & {$[0.134,1.396]$} & B & 56 & 4.461 & {$[2.518,5.762]$} & {$[1.386,7.820]$} & A \\
\hline 26 & 0.167 & {$[0.082,0.262]$} & {$[0.043,0.445]$} & B & 57 & 0.398 & {$[0.198,0.633]$} & {$[0.101,1.055]$} & B \\
\hline 27 & 1.826 & {$[0.975,3.067]$} & {$[0.468,4.768]$} & B & 63 & 0.032 & {$[0.016,0.051]$} & {$[0.008,0.088]$} & B \\
\hline 28 & 0.195 & {$[0.097,0.311]$} & {$[0.050,0.524]$} & B & 65 & 0.059 & {$[0.029,0.094]$} & {$[0.015,0.160]$} & B \\
\hline 29 & 0.826 & {$[0.420,1.336]$} & {$[0.211,2.172]$} & B & 66 & 0.026 & {$[0.013,0.041]$} & {$[0.007,0.071]$} & B \\
\hline 37 & 0.061 & {$[0.030,0.096]$} & {$[0.016,0.164]$} & B & 67 & 0.142 & {$[0.071,0.226]$} & {$[0.036,0.381]$} & B \\
\hline 38 & 0.232 & {$[0.115,0.368]$} & {$[0.059,0.618]$} & B & 68 & 0.029 & {$[0.014,0.046]$} & {$[0.007,0.078]$} & B \\
\hline 39 & 2.167 & {$[1.176,3.662]$} & {$[0.554,5.587]$} & B & 69 & 0.083 & {$[0.041,0.131]$} & {$[0.021,0.223]$} & B \\
\hline 40 & 0.519 & {$[0.262,0.836]$} & {$[0.133,1.376]$} & B & 70 & 0.444 & {$[0.223,0.711]$} & {$[0.113,1.177]$} & B \\
\hline 41 & 3.364 & {$[1.347,4.419]$} & {$[0.512,6.527]$} & A & 71 & 0.023 & {$[0.011,0.036]$} & {$[0.006,0.063]$} & B \\
\hline 42 & 0.338 & {$[0.168,0.538]$} & {$[0.086,0.902]$} & B & 77 & 0.030 & {$[0.015,0.047]$} & {$[0.008,0.082]$} & B \\
\hline 43 & 2.648 & {$[0.565,3.420]$} & {$[0.090,5.652]$} & A & 79 & 0.027 & {$[0.013,0.043]$} & {$[0.007,0.074]$} & B \\
\hline 44 & 0.094 & {$[0.046,0.148]$} & {$[0.024,0.253]$} & B & 81 & 0.017 & {$[0.008,0.026]$} & {$[0.004,0.045]$} & B \\
\hline 50 & 0.116 & {$[0.057,0.183]$} & {$[0.030,0.311]$} & B & 83 & 0.189 & {$[0.094,0.300]$} & {$[0.048,0.507]$} & B \\
\hline 51 & 0.179 & {$[0.088,0.284]$} & {$[0.046,0.481]$} & B & 84 & 0.013 & {$[0.006,0.020]$} & {$[0.003,0.034]$} & B \\
\hline 52 & 0.071 & {$[0.035,0.111]$} & {$[0.018,0.191]$} & B & 98 & 0.192 & {$[0.095,0.304]$} & {$[0.049,0.514]$} & B \\
\hline 53 & 0.305 & {$[0.151,0.483]$} & {$[0.078,0.811]$} & B & 99 & 0.013 & {$[0.006,0.020]$} & {$[0.003,0.036]$} & B \\
\hline 54 & 0.043 & {$[0.021,0.067]$} & {$[0.011,0.115]$} & B & & & & & \\
\hline
\end{tabular}

Table 35. Partial ionization cross sections $\left(10^{-20} \mathrm{~m}^{2}\right)$ for 3,4-dimethyl-1-pentene. TICS $/ 10^{-20} \mathrm{~m}^{2}=21.402 . M=98$, base peak is $\mathrm{m} / \mathrm{z}$ 56.

\begin{tabular}{|c|c|c|c|c|c|c|c|c|c|}
\hline$m / z$ & PICS & $67 \%$ interval & 95\% interval & unc. model & $m / \mathbf{z}$ & PICS & $67 \%$ interval & $95 \%$ interval & unc. model \\
\hline 1 & 0.400 & {$[0.289,0.479]$} & {$[0.214,0.599]$} & $\mathrm{H}$ & 58 & 0.005 & {$[0.002,0.008]$} & {$[0.001,0.013]$} & B \\
\hline 12 & 0.016 & {$[0.008,0.025]$} & {$[0.004,0.043]$} & B & 60 & 0.002 & {$[0.001,0.004]$} & {$[0.001,0.006]$} & B \\
\hline 13 & 0.030 & {$[0.015,0.047]$} & {$[0.008,0.081]$} & B & 61 & 0.005 & {$[0.002,0.007]$} & {$[0.001,0.012]$} & B \\
\hline 14 & 0.123 & {$[0.061,0.196]$} & {$[0.031,0.332]$} & B & 62 & 0.011 & {$[0.005,0.017]$} & {$[0.003,0.030]$} & B \\
\hline 15 & 0.897 & {$[0.454,1.438]$} & {$[0.229,2.350]$} & B & 63 & 0.024 & {$[0.012,0.038]$} & {$[0.006,0.065]$} & B \\
\hline 16 & 0.040 & {$[0.020,0.063]$} & {$[0.010,0.108]$} & B & 64 & 0.004 & {$[0.002,0.007]$} & {$[0.001,0.012]$} & B \\
\hline 25 & 0.010 & {$[0.005,0.016]$} & {$[0.003,0.027]$} & B & 65 & 0.038 & {$[0.018,0.059]$} & {$[0.010,0.103]$} & B \\
\hline 26 & 0.246 & {$[0.122,0.389]$} & {$[0.063,0.653]$} & B & 66 & 0.019 & {$[0.009,0.029]$} & {$[0.005,0.050]$} & B \\
\hline 27 & 2.793 & {$[0.758,3.625]$} & {$[0.171,5.774]$} & A & 67 & 0.102 & {$[0.050,0.161]$} & {$[0.026,0.277]$} & B \\
\hline 28 & 0.528 & {$[0.266,0.847]$} & {$[0.134,1.392]$} & B & 68 & 0.024 & {$[0.011,0.037]$} & {$[0.006,0.064]$} & B \\
\hline 29 & 1.217 & {$[0.632,2.001]$} & {$[0.312,3.196]$} & B & 69 & 0.188 & {$[0.093,0.296]$} & {$[0.048,0.500]$} & B \\
\hline 30 & 0.027 & {$[0.013,0.042]$} & {$[0.007,0.072]$} & B & 70 & 1.108 & {$[0.567,1.803]$} & {$[0.283,2.922]$} & B \\
\hline 31 & 0.004 & {$[0.002,0.007]$} & {$[0.001,0.012]$} & B & 71 & 0.064 & {$[0.031,0.100]$} & {$[0.016,0.171]$} & B \\
\hline 32 & 0.004 & {$[0.002,0.007]$} & {$[0.001,0.012]$} & B & 72 & 0.002 & {$[0.001,0.003]$} & {$[0.000,0.005]$} & B \\
\hline 36 & 0.004 & {$[0.002,0.006]$} & {$[0.001,0.010]$} & B & 73 & 0.002 & {$[0.001,0.003]$} & {$[0.000,0.005]$} & B \\
\hline 37 & 0.049 & {$[0.024,0.077]$} & {$[0.013,0.133]$} & B & 74 & 0.003 & {$[0.002,0.005]$} & {$[0.001,0.009]$} & B \\
\hline 38 & 0.145 & {$[0.071,0.228]$} & {$[0.037,0.387]$} & B & 75 & 0.002 & {$[0.001,0.003]$} & {$[0.000,0.004]$} & B \\
\hline 39 & 1.545 & {$[0.813,2.556]$} & {$[0.399,4.044]$} & B & 76 & 0.002 & {$[0.001,0.002]$} & {$[0.000,0.004]$} & B \\
\hline 40 & 0.239 & {$[0.119,0.381]$} & {$[0.061,0.637]$} & B & 77 & 0.014 & {$[0.007,0.022]$} & {$[0.004,0.037]$} & B \\
\hline 41 & 2.526 & {$[1.412,4.328]$} & {$[0.653,6.463]$} & B & 78 & 0.003 & {$[0.001,0.005]$} & {$[0.001,0.008]$} & B \\
\hline 42 & 0.321 & {$[0.160,0.512]$} & {$[0.083,0.859]$} & B & 79 & 0.012 & {$[0.006,0.018]$} & {$[0.003,0.031]$} & B \\
\hline 43 & 2.470 & {$[1.377,4.234]$} & {$[0.639,6.328]$} & B & 80 & 0.003 & {$[0.001,0.004]$} & {$[0.001,0.008]$} & B \\
\hline 44 & 0.078 & {$[0.038,0.123]$} & {$[0.020,0.211]$} & B & 81 & 0.014 & {$[0.007,0.021]$} & {$[0.003,0.037]$} & B \\
\hline 45 & 0.003 & {$[0.002,0.005]$} & {$[0.001,0.009]$} & B & 82 & 0.003 & {$[0.001,0.004]$} & {$[0.001,0.007]$} & B \\
\hline 46 & 0.003 & {$[0.002,0.005]$} & {$[0.001,0.009]$} & B & 83 & 0.151 & {$[0.074,0.238]$} & {$[0.039,0.403]$} & B \\
\hline 49 & 0.012 & {$[0.006,0.018]$} & {$[0.003,0.032]$} & B & 84 & 0.010 & {$[0.005,0.015]$} & {$[0.002,0.026]$} & B \\
\hline 50 & 0.104 & {$[0.051,0.165]$} & {$[0.027,0.279]$} & B & 85 & 0.001 & {$[0.001,0.002]$} & {$[0.000,0.003]$} & B \\
\hline 51 & 0.165 & {$[0.082,0.261]$} & {$[0.042,0.442]$} & B & 91 & 0.001 & {$[0.001,0.002]$} & {$[0.000,0.003]$} & B \\
\hline 52 & 0.061 & {$[0.030,0.096]$} & {$[0.016,0.164]$} & B & 95 & 0.001 & {$[0.001,0.002]$} & {$[0.000,0.003]$} & B \\
\hline 53 & 0.321 & {$[0.159,0.510]$} & {$[0.082,0.860]$} & B & 96 & 0.001 & {$[0.001,0.002]$} & {$[0.000,0.003]$} & B \\
\hline 54 & 0.325 & {$[0.160,0.513]$} & {$[0.083,0.864]$} & B & 97 & 0.006 & {$[0.003,0.009]$} & {$[0.001,0.015]$} & B \\
\hline 55 & 2.155 & {$[1.171,3.653]$} & {$[0.550,5.550]$} & B & 98 & 0.009 & {$[0.004,0.014]$} & {$[0.002,0.025]$} & B \\
\hline 56 & 2.556 & {$[0.489,3.255]$} & {$[0.064,5.483]$} & A & 99 & 0.001 & {$[0.001,0.002]$} & {$[0.000,0.003]$} & B \\
\hline 57 & 0.153 & {$[0.075,0.240]$} & {$[0.039,0.408]$} & B & & & & & \\
\hline
\end{tabular}


Table 36. Partial ionization cross sections $\left(10^{-20} \mathrm{~m}^{2}\right)$ for 3,3-dimethyl-1-pentene. TICS $/ 10^{-20} \mathrm{~m}^{2}=21 \cdot 137 . M=98$, base peak is $\mathrm{m} / \mathrm{z}$ 69.

\begin{tabular}{|c|c|c|c|c|c|c|c|c|c|}
\hline$m / z$ & PICS & $67 \%$ interval & $95 \%$ interval & unc. model & $m / z$ & PICS & $67 \%$ interval & $95 \%$ interval & unc. model \\
\hline 1 & 0.400 & {$[0.288,0.478]$} & {$[0.215,0.599]$} & $\mathrm{H}$ & 56 & 0.633 & {$[0.318,1.015]$} & {$[0.162,1.675]$} & B \\
\hline 13 & 0.047 & {$[0.023,0.074]$} & {$[0.012,0.127]$} & B & 58 & 0.004 & {$[0.002,0.006]$} & {$[0.001,0.010]$} & B \\
\hline 15 & 0.941 & {$[0.482,1.535]$} & {$[0.240,2.490]$} & B & 61 & 0.007 & {$[0.003,0.011]$} & {$[0.002,0.019]$} & B \\
\hline 16 & 0.021 & {$[0.010,0.032]$} & {$[0.005,0.056]$} & B & 62 & 0.017 & {$[0.009,0.027]$} & {$[0.004,0.047]$} & B \\
\hline 24 & 0.008 & {$[0.004,0.012]$} & {$[0.002,0.022]$} & B & 63 & 0.031 & {$[0.015,0.048]$} & {$[0.008,0.083]$} & B \\
\hline 26 & 0.313 & {$[0.155,0.495]$} & {$[0.080,0.832]$} & B & 65 & 0.052 & {$[0.026,0.082]$} & {$[0.014,0.142]$} & B \\
\hline 27 & 2.593 & {$[0.541,3.356]$} & {$[0.079,5.526]$} & A & 66 & 0.026 & {$[0.012,0.040]$} & {$[0.007,0.069]$} & B \\
\hline 28 & 0.334 & {$[0.168,0.536]$} & {$[0.086,0.894]$} & B & 67 & 0.181 & {$[0.090,0.288]$} & {$[0.047,0.489]$} & B \\
\hline 29 & 1.049 & {$[0.537,1.708]$} & {$[0.266,2.745]$} & B & 68 & 0.119 & {$[0.059,0.188]$} & {$[0.030,0.318]$} & B \\
\hline 30 & 0.021 & {$[0.010,0.033]$} & {$[0.005,0.056]$} & B & 69 & 2.976 & {$[0.984,3.919]$} & {$[0.284,5.989]$} & A \\
\hline 31 & 0.007 & {$[0.003,0.010]$} & {$[0.002,0.018]$} & B & 70 & 0.346 & {$[0.173,0.552]$} & {$[0.088,0.919]$} & B \\
\hline 38 & 0.150 & {$[0.074,0.237]$} & {$[0.038,0.400]$} & B & 74 & 0.003 & {$[0.001,0.004]$} & {$[0.001,0.007]$} & B \\
\hline 39 & 1.628 & {$[0.864,2.725]$} & {$[0.414,4.238]$} & B & 75 & 0.003 & {$[0.001,0.004]$} & {$[0.001,0.007]$} & B \\
\hline 40 & 0.243 & {$[0.121,0.387]$} & {$[0.062,0.652]$} & B & 77 & 0.012 & {$[0.006,0.019]$} & {$[0.003,0.032]$} & B \\
\hline 41 & 4.388 & {$[2.479,5.655]$} & {$[1.369,7.660]$} & A & 78 & 0.002 & {$[0.001,0.004]$} & {$[0.001,0.006]$} & B \\
\hline 42 & 0.270 & {$[0.135,0.432]$} & {$[0.070,0.727]$} & B & 79 & 0.011 & {$[0.005,0.018]$} & {$[0.003,0.030]$} & B \\
\hline 43 & 0.644 & {$[0.327,1.041]$} & {$[0.165,1.704]$} & B & 80 & 0.002 & {$[0.001,0.003]$} & {$[0.001,0.006]$} & B \\
\hline 44 & 0.020 & {$[0.010,0.032]$} & {$[0.005,0.055]$} & B & 81 & 0.015 & {$[0.007,0.023]$} & {$[0.004,0.040]$} & B \\
\hline 45 & 0.005 & {$[0.002,0.008]$} & {$[0.001,0.014]$} & B & 82 & 0.004 & {$[0.002,0.006]$} & {$[0.001,0.011]$} & B \\
\hline 46 & 0.005 & {$[0.002,0.008]$} & {$[0.001,0.013]$} & B & 83 & 0.322 & {$[0.160,0.511]$} & {$[0.082,0.858]$} & B \\
\hline 48 & 0.005 & {$[0.002,0.007]$} & {$[0.001,0.013]$} & B & 84 & 0.021 & {$[0.010,0.032]$} & {$[0.005,0.055]$} & B \\
\hline 49 & 0.014 & {$[0.007,0.022]$} & {$[0.004,0.037]$} & B & 85 & 0.002 & {$[0.001,0.003]$} & {$[0.000,0.005]$} & B \\
\hline
\end{tabular}


Table 37. Partial ionization cross sections $\left(10^{-20} \mathrm{~m}^{2}\right)$ for 4,4-dimethyl-1-pentene. TICS $/ 10^{-20} \mathrm{~m}^{2}=20.652 . M=98$, base peak is $\mathrm{m} / \mathrm{z}$ 57.

\begin{tabular}{|c|c|c|c|c|c|c|c|c|c|}
\hline$m / z$ & PICS & $67 \%$ interval & $95 \%$ interval & unc. model & $m / z$ & PICS & $67 \%$ interval & $95 \%$ interval & unc. model \\
\hline 1 & 0.400 & {$[0.288,0.478]$} & {$[0.215,0.600]$} & $\mathrm{H}$ & 58 & 0.315 & {$[0.156,0.499]$} & {$[0.081,0.842]$} & B \\
\hline 15 & 0.152 & {$[0.075,0.242]$} & {$[0.039,0.410]$} & B & 59 & 0.039 & {$[0.019,0.062]$} & {$[0.010,0.107]$} & B \\
\hline 26 & 0.067 & {$[0.033,0.106]$} & {$[0.017,0.179]$} & B & 62 & 0.006 & {$[0.003,0.010]$} & {$[0.002,0.017]$} & B \\
\hline 27 & 0.773 & {$[0.391,1.239]$} & {$[0.197,2.021]$} & B & 63 & 0.018 & {$[0.009,0.028]$} & {$[0.005,0.049]$} & B \\
\hline 28 & 0.051 & {$[0.025,0.081]$} & {$[0.013,0.139]$} & B & 65 & 0.035 & {$[0.017,0.054]$} & {$[0.009,0.093]$} & B \\
\hline 29 & 2.508 & {$[0.502,3.241]$} & {$[0.066,5.367]$} & A & 66 & 0.011 & {$[0.005,0.018]$} & {$[0.003,0.030]$} & B \\
\hline 30 & 0.049 & {$[0.024,0.077]$} & {$[0.013,0.132]$} & B & 67 & 0.099 & {$[0.048,0.155]$} & {$[0.025,0.265]$} & B \\
\hline 31 & 0.012 & {$[0.006,0.019]$} & {$[0.003,0.032]$} & B & 68 & 0.016 & {$[0.008,0.025]$} & {$[0.004,0.044]$} & B \\
\hline 37 & 0.021 & {$[0.010,0.033]$} & {$[0.005,0.056]$} & B & 69 & 0.031 & {$[0.015,0.049]$} & {$[0.008,0.084]$} & B \\
\hline 38 & 0.081 & {$[0.040,0.128]$} & {$[0.021,0.218]$} & B & 70 & 0.138 & {$[0.068,0.217]$} & {$[0.036,0.368]$} & B \\
\hline 39 & 1.094 & {$[0.568,1.801]$} & {$[0.280,2.876]$} & B & 71 & 0.020 & {$[0.010,0.031]$} & {$[0.005,0.054]$} & B \\
\hline 40 & 0.165 & {$[0.081,0.259]$} & {$[0.042,0.439]$} & B & 77 & 0.013 & {$[0.006,0.020]$} & {$[0.003,0.034]$} & B \\
\hline 41 & 3.711 & {$[1.842,4.857]$} & {$[0.863,6.802]$} & A & 79 & 0.012 & {$[0.006,0.019]$} & {$[0.003,0.032]$} & B \\
\hline 42 & 0.186 & {$[0.091,0.292]$} & {$[0.047,0.496]$} & B & 81 & 0.037 & {$[0.018,0.057]$} & {$[0.009,0.098]$} & B \\
\hline 43 & 0.264 & {$[0.132,0.424]$} & {$[0.068,0.709]$} & B & 82 & 0.018 & {$[0.009,0.028]$} & {$[0.005,0.049]$} & B \\
\hline 44 & 0.009 & {$[0.004,0.014]$} & {$[0.002,0.024]$} & B & 83 & 0.523 & {$[0.263,0.840]$} & {$[0.134,1.392]$} & B \\
\hline 50 & 0.048 & {$[0.023,0.075]$} & {$[0.012,0.128]$} & B & 84 & 0.036 & {$[0.018,0.057]$} & {$[0.009,0.097]$} & B \\
\hline 51 & 0.070 & {$[0.034,0.110]$} & {$[0.018,0.186]$} & B & 85 & 0.003 & {$[0.002,0.005]$} & {$[0.001,0.009]$} & B \\
\hline 52 & 0.023 & {$[0.011,0.036]$} & {$[0.006,0.062]$} & B & 95 & 0.006 & {$[0.003,0.010]$} & {$[0.002,0.017]$} & B \\
\hline 53 & 0.156 & {$[0.077,0.248]$} & {$[0.040,0.420]$} & B & 96 & 0.003 & {$[0.002,0.005]$} & {$[0.001,0.008]$} & B \\
\hline 54 & 0.029 & {$[0.014,0.046]$} & {$[0.007,0.079]$} & B & 97 & 0.009 & {$[0.005,0.015]$} & {$[0.002,0.025]$} & B \\
\hline 55 & 2.053 & {$[1.117,3.487]$} & {$[0.522,5.293]$} & B & 98 & 0.034 & {$[0.017,0.054]$} & {$[0.009,0.093]$} & B \\
\hline 56 & 0.434 & {$[0.217,0.695]$} & {$[0.110,1.150]$} & B & 99 & 0.013 & {$[0.006,0.020]$} & {$[0.003,0.034]$} & B \\
\hline 57 & 6.862 & {$[4.915,8.340]$} & {$[3.541,10.421]$} & A & & & & & \\
\hline
\end{tabular}

Table 38. Partial ionization cross sections $\left(10^{-20} \mathrm{~m}^{2}\right)$ for 2,3-dimethyl-2-pentene. TICS $/ 10^{-20} \mathrm{~m}^{2}=21.773 . M=98$, base peak is $\mathrm{m} / \mathrm{z}$ 55.

\begin{tabular}{|c|c|c|c|c|c|c|c|c|c|}
\hline$m / z$ & PICS & $67 \%$ interval & $95 \%$ interval & unc. model & $m / z$ & PICS & $67 \%$ interval & $95 \%$ interval & unc. model \\
\hline 1 & 0.400 & {$[0.289,0.479]$} & {$[0.215,0.599]$} & $\mathrm{H}$ & 61 & 0.004 & {$[0.002,0.006]$} & {$[0.001,0.011]$} & B \\
\hline 14 & 0.026 & {$[0.013,0.041]$} & {$[0.007,0.070]$} & B & 62 & 0.012 & {$[0.006,0.019]$} & {$[0.003,0.033]$} & B \\
\hline 15 & 0.274 & {$[0.136,0.434]$} & {$[0.070,0.729]$} & B & 63 & 0.024 & {$[0.012,0.037]$} & {$[0.006,0.064]$} & B \\
\hline 26 & 0.080 & {$[0.039,0.125]$} & {$[0.020,0.213]$} & B & 64 & 0.004 & {$[0.002,0.006]$} & {$[0.001,0.011]$} & B \\
\hline 27 & 1.217 & {$[0.634,2.006]$} & {$[0.311,3.195]$} & B & 65 & 0.064 & {$[0.031,0.101]$} & {$[0.016,0.173]$} & B \\
\hline 28 & 0.076 & {$[0.037,0.119]$} & {$[0.019,0.204]$} & B & 66 & 0.026 & {$[0.013,0.041]$} & {$[0.007,0.070]$} & B \\
\hline 29 & 1.051 & {$[0.547,1.738]$} & {$[0.269,2.771]$} & B & 67 & 0.264 & {$[0.131,0.420]$} & {$[0.068,0.707]$} & B \\
\hline 30 & 0.024 & {$[0.012,0.038]$} & {$[0.006,0.065]$} & B & 68 & 0.056 & {$[0.028,0.088]$} & {$[0.014,0.151]$} & B \\
\hline 31 & 0.101 & {$[0.050,0.160]$} & {$[0.026,0.272]$} & B & 69 & 0.728 & {$[0.370,1.180]$} & {$[0.186,1.928]$} & B \\
\hline 36 & 0.014 & {$[0.007,0.022]$} & {$[0.004,0.038]$} & B & 70 & 0.151 & {$[0.075,0.240]$} & {$[0.039,0.410]$} & B \\
\hline 37 & 0.034 & {$[0.017,0.054]$} & {$[0.009,0.092]$} & B & 71 & 0.033 & {$[0.016,0.051]$} & {$[0.008,0.088]$} & B \\
\hline 38 & 0.073 & {$[0.036,0.115]$} & {$[0.019,0.197]$} & B & 72 & 0.006 & {$[0.003,0.010]$} & {$[0.002,0.017]$} & B \\
\hline 39 & 1.172 & {$[0.608,1.930]$} & {$[0.299,3.080]$} & B & 73 & 0.003 & {$[0.002,0.005]$} & {$[0.001,0.008]$} & B \\
\hline 40 & 0.217 & {$[0.108,0.345]$} & {$[0.056,0.583]$} & B & 74 & 0.006 & {$[0.003,0.009]$} & {$[0.002,0.016]$} & B \\
\hline 41 & 4.101 & {$[2.152,5.299]$} & {$[1.072,7.273]$} & A & 77 & 0.025 & {$[0.012,0.039]$} & {$[0.006,0.067]$} & B \\
\hline 42 & 0.313 & {$[0.157,0.503]$} & {$[0.081,0.843]$} & B & 78 & 0.005 & {$[0.003,0.008]$} & {$[0.001,0.015]$} & B \\
\hline 43 & 1.138 & {$[0.587,1.862]$} & {$[0.291,2.995]$} & B & 79 & 0.039 & {$[0.019,0.061]$} & {$[0.010,0.105]$} & B \\
\hline 44 & 0.035 & {$[0.017,0.055]$} & {$[0.009,0.095]$} & B & 80 & 0.005 & {$[0.002,0.008]$} & {$[0.001,0.014]$} & B \\
\hline 45 & 0.052 & {$[0.025,0.081]$} & {$[0.013,0.140]$} & B & 81 & 0.102 & {$[0.050,0.161]$} & {$[0.026,0.273]$} & B \\
\hline 46 & 0.006 & {$[0.003,0.009]$} & {$[0.001,0.015]$} & B & 82 & 0.021 & {$[0.010,0.033]$} & {$[0.005,0.058]$} & B \\
\hline 49 & 0.005 & {$[0.003,0.008]$} & {$[0.001,0.014]$} & B & 83 & 1.877 & {$[1.021,3.195]$} & {$[0.483,4.907]$} & B \\
\hline 50 & 0.067 & {$[0.033,0.106]$} & {$[0.017,0.182]$} & B & 84 & 0.124 & {$[0.060,0.194]$} & {$[0.031,0.329]$} & B \\
\hline 51 & 0.127 & {$[0.063,0.202]$} & {$[0.033,0.344]$} & B & 85 & 0.100 & {$[0.049,0.158]$} & {$[0.026,0.269]$} & B \\
\hline 52 & 0.060 & {$[0.029,0.093]$} & {$[0.015,0.159]$} & B & 86 & 0.006 & {$[0.003,0.010]$} & {$[0.002,0.017]$} & B \\
\hline 53 & 0.373 & {$[0.186,0.594]$} & {$[0.095,0.993]$} & B & 91 & 0.002 & {$[0.001,0.003]$} & {$[0.001,0.006]$} & B \\
\hline 54 & 0.110 & {$[0.054,0.174]$} & {$[0.028,0.295]$} & B & 95 & 0.006 & {$[0.003,0.010]$} & {$[0.002,0.017]$} & B \\
\hline 55 & 4.710 & {$[2.764,5.993]$} & {$[1.580,7.983]$} & A & 96 & 0.014 & {$[0.007,0.022]$} & {$[0.004,0.039]$} & B \\
\hline 56 & 0.632 & {$[0.318,1.014]$} & {$[0.161,1.672]$} & B & 97 & 0.019 & {$[0.009,0.029]$} & {$[0.005,0.050]$} & B \\
\hline 57 & 0.207 & {$[0.102,0.326]$} & {$[0.053,0.550]$} & B & 98 & 0.778 & {$[0.394,1.256]$} & {$[0.198,2.051]$} & B \\
\hline 58 & 0.079 & {$[0.039,0.124]$} & {$[0.020,0.210]$} & B & 99 & 0.084 & {$[0.041,0.133]$} & {$[0.022,0.228]$} & B \\
\hline 59 & 0.385 & {$[0.192,0.614]$} & {$[0.099,1.022]$} & B & 100 & 0.008 & {$[0.004,0.013]$} & {$[0.002,0.022]$} & B \\
\hline 60 & 0.013 & {$[0.006,0.020]$} & {$[0.003,0.034]$} & B & & & & & \\
\hline
\end{tabular}


Table 39. Partial ionization cross sections $\left(10^{-20} \mathrm{~m}^{2}\right)$ for 2,4-dimethyl-2-pentene. TICS $/ 10^{-20} \mathrm{~m}^{2}=20.712 . M=98$, base peak is $\mathrm{m} / \mathrm{z}$ 55.

\begin{tabular}{|c|c|c|c|c|c|c|c|c|c|}
\hline$m / z$ & PICS & $67 \%$ interval & $95 \%$ interval & unc. model & $m / z$ & PICS & $67 \%$ interval & $95 \%$ interval & unc. model \\
\hline 1 & 0.400 & {$[0.288,0.478]$} & {$[0.216,0.600]$} & $\mathrm{H}$ & 54 & 0.087 & {$[0.043,0.138]$} & {$[0.023,0.237]$} & B \\
\hline 26 & 0.054 & {$[0.026,0.085]$} & {$[0.014,0.145]$} & B & 56 & 0.475 & {$[0.237,0.759]$} & {$[0.121,1.259]$} & B \\
\hline 28 & 0.204 & {$[0.101,0.323]$} & {$[0.052,0.546]$} & B & 65 & 0.092 & {$[0.045,0.144]$} & {$[0.023,0.245]$} & B \\
\hline 29 & 1.046 & {$[0.538,1.708]$} & {$[0.266,2.734]$} & B & 67 & 0.307 & {$[0.154,0.491]$} & {$[0.079,0.819]$} & B \\
\hline 38 & 0.081 & {$[0.040,0.127]$} & {$[0.021,0.217]$} & B & 69 & 0.146 & {$[0.073,0.233]$} & {$[0.038,0.396]$} & B \\
\hline 40 & 0.233 & {$[0.116,0.372]$} & {$[0.060,0.626]$} & B & 77 & 0.034 & {$[0.016,0.052]$} & {$[0.009,0.090]$} & B \\
\hline 41 & 2.968 & {$[1.022,3.911]$} & {$[0.317,5.939]$} & A & 79 & 0.047 & {$[0.023,0.075]$} & {$[0.012,0.129]$} & B \\
\hline 42 & 0.186 & {$[0.092,0.296]$} & {$[0.047,0.497]$} & B & 81 & 0.044 & {$[0.022,0.069]$} & {$[0.011,0.120]$} & B \\
\hline 43 & 1.533 & {$[0.811,2.563]$} & {$[0.391,4.009]$} & B & 82 & 0.043 & {$[0.021,0.067]$} & {$[0.011,0.114]$} & B \\
\hline 44 & 0.072 & {$[0.035,0.112]$} & {$[0.018,0.191]$} & B & 83 & 2.538 & {$[0.534,3.288]$} & {$[0.073,5.407]$} & A \\
\hline 50 & 0.063 & {$[0.031,0.099]$} & {$[0.016,0.170]$} & B & 84 & 0.145 & {$[0.072,0.230]$} & {$[0.037,0.389]$} & B \\
\hline
\end{tabular}

Table 40. Partial ionization cross sections $\left(10^{-20} \mathrm{~m}^{2}\right)$ for 3,4-dimethyl-2-pentene. TICS $/ 10^{-20} \mathrm{~m}^{2}=20.574 . M=98$, base peak is $m / \mathrm{z}$ 83.

\begin{tabular}{|c|c|c|c|c|c|c|c|c|c|}
\hline$m / z$ & PICS & $67 \%$ interval & $95 \%$ interval & unc. model & $m / z$ & PICS & $67 \%$ interval & $95 \%$ interval & unc. model \\
\hline 1 & 0.400 & {$[0.289,0.479]$} & {$[0.215,0.599]$} & $\mathrm{H}$ & 61 & 0.014 & {$[0.007,0.021]$} & {$[0.003,0.036]$} & B \\
\hline 14 & 0.028 & {$[0.014,0.045]$} & {$[0.007,0.077]$} & B & 62 & 0.022 & {$[0.011,0.034]$} & {$[0.006,0.060]$} & B \\
\hline 15 & 0.284 & {$[0.142,0.456]$} & {$[0.073,0.765]$} & B & 63 & 0.043 & {$[0.021,0.067]$} & {$[0.011,0.115]$} & B \\
\hline 16 & 0.013 & {$[0.006,0.020]$} & {$[0.003,0.035]$} & B & 64 & 0.008 & {$[0.004,0.013]$} & {$[0.002,0.023]$} & B \\
\hline 26 & 0.124 & {$[0.061,0.195]$} & {$[0.032,0.330]$} & B & 65 & 0.090 & {$[0.044,0.142]$} & {$[0.023,0.243]$} & B \\
\hline 27 & 1.378 & {$[0.718,2.282]$} & {$[0.350,3.616]$} & B & 66 & 0.032 & {$[0.016,0.050]$} & {$[0.008,0.087]$} & B \\
\hline 28 & 0.145 & {$[0.072,0.230]$} & {$[0.038,0.392]$} & B & 67 & 0.354 & {$[0.177,0.565]$} & {$[0.091,0.943]$} & B \\
\hline 29 & 0.856 & {$[0.436,1.389]$} & {$[0.218,2.258]$} & B & 68 & 0.053 & {$[0.026,0.084]$} & {$[0.014,0.145]$} & B \\
\hline 30 & 0.017 & {$[0.008,0.027]$} & {$[0.004,0.047]$} & B & 69 & 0.446 & {$[0.223,0.713]$} & {$[0.114,1.188]$} & B \\
\hline 36 & 0.015 & {$[0.007,0.024]$} & {$[0.004,0.041]$} & B & 70 & 0.164 & {$[0.081,0.260]$} & {$[0.042,0.440]$} & B \\
\hline 37 & 0.037 & {$[0.018,0.058]$} & {$[0.010,0.101]$} & B & 71 & 0.039 & {$[0.019,0.061]$} & {$[0.010,0.104]$} & B \\
\hline 38 & 0.108 & {$[0.053,0.171]$} & {$[0.028,0.291]$} & B & 72 & 0.007 & {$[0.003,0.011]$} & {$[0.002,0.019]$} & B \\
\hline 39 & 1.330 & {$[0.696,2.202]$} & {$[0.339,3.476]$} & B & 74 & 0.010 & {$[0.005,0.015]$} & {$[0.003,0.027]$} & B \\
\hline 40 & 0.194 & {$[0.096,0.308]$} & {$[0.050,0.521]$} & B & 75 & 0.003 & {$[0.002,0.005]$} & {$[0.001,0.009]$} & B \\
\hline 41 & 2.837 & {$[0.890,3.730]$} & {$[0.237,5.741]$} & A & 77 & 0.036 & {$[0.017,0.056]$} & {$[0.009,0.097]$} & B \\
\hline 42 & 0.253 & {$[0.125,0.400]$} & {$[0.065,0.676]$} & B & 78 & 0.006 & {$[0.003,0.009]$} & {$[0.002,0.016]$} & B \\
\hline 43 & 1.053 & {$[0.544,1.729]$} & {$[0.269,2.778]$} & B & 79 & 0.042 & {$[0.021,0.066]$} & {$[0.011,0.114]$} & B \\
\hline 44 & 0.032 & {$[0.016,0.050]$} & {$[0.008,0.087]$} & B & 80 & 0.008 & {$[0.004,0.013]$} & {$[0.002,0.022]$} & B \\
\hline 45 & 0.013 & {$[0.006,0.020]$} & {$[0.003,0.034]$} & B & 81 & 0.087 & {$[0.043,0.137]$} & {$[0.022,0.235]$} & B \\
\hline 49 & 0.017 & {$[0.008,0.027]$} & {$[0.004,0.047]$} & B & 82 & 0.046 & {$[0.022,0.072]$} & {$[0.012,0.124]$} & B \\
\hline 50 & 0.130 & {$[0.064,0.205]$} & {$[0.033,0.349]$} & B & 83 & 2.459 & {$[0.467,3.174]$} & {$[0.054,5.286]$} & A \\
\hline 51 & 0.222 & {$[0.111,0.354]$} & {$[0.057,0.597]$} & B & 84 & 0.149 & {$[0.073,0.235]$} & {$[0.038,0.399]$} & B \\
\hline 52 & 0.087 & {$[0.042,0.136]$} & {$[0.022,0.233]$} & B & 85 & 0.007 & {$[0.003,0.011]$} & {$[0.002,0.018]$} & B \\
\hline 53 & 0.463 & {$[0.232,0.743]$} & {$[0.119,1.240]$} & B & 86 & 0.004 & {$[0.002,0.007]$} & {$[0.001,0.012]$} & B \\
\hline 54 & 0.146 & {$[0.072,0.229]$} & {$[0.037,0.387]$} & B & 95 & 0.002 & {$[0.001,0.003]$} & {$[0.001,0.006]$} & B \\
\hline 55 & 4.773 & {$[2.908,6.077]$} & {$[1.757,8.049]$} & A & 96 & 0.004 & {$[0.002,0.007]$} & {$[0.001,0.012]$} & B \\
\hline 56 & 0.460 & {$[0.232,0.741]$} & {$[0.119,1.232]$} & B & 97 & 0.020 & {$[0.010,0.032]$} & {$[0.005,0.055]$} & B \\
\hline 57 & 0.073 & {$[0.036,0.114]$} & {$[0.019,0.197]$} & B & 98 & 0.843 & {$[0.429,1.367]$} & {$[0.216,2.233]$} & B \\
\hline 58 & 0.005 & {$[0.002,0.008]$} & {$[0.001,0.013]$} & B & 99 & 0.067 & {$[0.033,0.105]$} & {$[0.017,0.181]$} & B \\
\hline 59 & 0.009 & {$[0.005,0.015]$} & {$[0.002,0.026]$} & B & 100 & 0.002 & {$[0.001,0.003]$} & {$[0.001,0.006]$} & B \\
\hline
\end{tabular}


Table 41. Partial ionization cross sections $\left(10^{-20} \mathrm{~m}^{2}\right)$ for 4,4-dimethyl-2-pentene. TICS $/ 10^{-20} \mathrm{~m}^{2}=21.590 . M=98$, base peak is $\mathrm{m} / \mathrm{z}$ 83.




Table 42. Partial ionization cross sections $\left(10^{-20} \mathrm{~m}^{2}\right)$ for 3-ethyl-1-pentene. TICS $/ 10^{-20} \mathrm{~m}^{2}=21.088 . M=98$, base peak is $\mathrm{m} / \mathrm{z} 41$.

\begin{tabular}{llllllllll}
\hline $\boldsymbol{m} / \mathbf{z}$ & $\mathbf{P I C S}$ & $\mathbf{6 7 \%}$ interval & $\mathbf{9 5 \%}$ interval & unc. model & $\mathbf{m} / \mathbf{z}$ & $\mathbf{P I C S}$ & $\mathbf{6 7 \%}$ interval & $\mathbf{9 5 \%}$ interval & unc. model \\
\hline 1 & 0.400 & {$[0.288,0.478]$} & {$[0.215,0.599]$} & $\mathrm{H}$ & 56 & 0.776 & {$[0.394,1.256]$} & {$[0.199,2.055]$} & $\mathrm{B}$ \\
12 & 0.012 & {$[0.006,0.019]$} & {$[0.003,0.032]$} & $\mathrm{B}$ & 57 & 0.095 & {$[0.047,0.150]$} & {$[0.025,0.257]$} & $\mathrm{B}$ \\
13 & 0.035 & {$[0.017,0.054]$} & {$[0.009,0.093]$} & $\mathrm{B}$ & 58 & 0.004 & {$[0.002,0.006]$} & {$[0.001,0.010]$} & $\mathrm{B}$ \\
14 & 0.099 & {$[0.049,0.156]$} & {$[0.025,0.264]$} & $\mathrm{B}$ & 60 & 0.004 & {$[0.002,0.006]$} & {$[0.001,0.010]$} & $\mathrm{B}$ \\
15 & 0.466 & {$[0.233,0.745]$} & {$[0.119,1.243]$} & $\mathrm{B}$ & 61 & 0.011 & {$[0.005,0.016]$} & {$[0.003,0.028]$} & $\mathrm{B}$ \\
16 & 0.010 & {$[0.005,0.016]$} & {$[0.003,0.027]$} & $\mathrm{B}$ & 62 & 0.021 & {$[0.010,0.032]$} & {$[0.005,0.055]$} & $\mathrm{B}$ \\
24 & 0.008 & {$[0.004,0.012]$} & {$[0.002,0.021]$} & $\mathrm{B}$ & 63 & 0.030 & {$[0.015,0.047]$} & {$[0.008,0.082]$} & $\mathrm{B}$ \\
25 & 0.023 & {$[0.011,0.036]$} & {$[0.006,0.063]$} & $\mathrm{B}$ & 64 & 0.003 & {$[0.002,0.005]$} & {$[0.001,0.009]$} & $\mathrm{B}$ \\
26 & 0.404 & {$[0.203,0.649]$} & {$[0.103,1.075]$} & $\mathrm{B}$ & 65 & 0.048 & {$[0.024,0.075]$} & {$[0.012,0.129]$} & $\mathrm{B}$ \\
27 & 2.695 & {$[0.685,3.467]$} & {$[0.136,5.510]$} & $\mathrm{A}$ & 66 & 0.022 & {$[0.011,0.034]$} & {$[0.006,0.059]$} & $\mathrm{B}$ \\
28 & 0.413 & {$[0.205,0.655]$} & {$[0.105,1.092]$} & $\mathrm{B}$ & 67 & 0.193 & {$[0.096,0.308]$} & {$[0.050,0.518]$} & $\mathrm{B}$ \\
29 & 1.125 & {$[0.581,1.842]$} & {$[0.290,2.970]$} & $\mathrm{B}$ & 68 & 0.072 & {$[0.035,0.113]$} & {$[0.018,0.194]$} & $\mathrm{B}$ \\
30 & 0.027 & {$[0.013,0.042]$} & {$[0.007,0.073]$} & $\mathrm{B}$ & 69 & 2.284 & {$[1.277,3.921]$} & {$[0.591,5.870]$} & $\mathrm{B}$ \\
31 & 0.013 & {$[0.006,0.021]$} & {$[0.003,0.036]$} & $\mathrm{B}$ & 70 & 0.610 & {$[0.307,0.978]$} & {$[0.155,1.604]$} & $\mathrm{B}$ \\
32 & 0.006 & {$[0.003,0.010]$} & {$[0.002,0.018]$} & $\mathrm{B}$ & 71 & 0.031 & {$[0.015,0.048]$} & {$[0.008,0.082]$} & $\mathrm{B}$ \\
36 & 0.006 & {$[0.003,0.009]$} & {$[0.002,0.016]$} & $\mathrm{B}$ & 72 & 0.003 & {$[0.001,0.004]$} & {$[0.001,0.007]$} & $\mathrm{B}$ \\
37 & 0.058 & {$[0.028,0.091]$} & {$[0.015,0.155]$} & $\mathrm{B}$ & 74 & 0.003 & {$[0.001,0.004]$} & {$[0.001,0.007]$} & $\mathrm{B}$ \\
38 & 0.164 & {$[0.081,0.259]$} & {$[0.042,0.441]$} & $\mathrm{B}$ & 75 & 0.002 & {$[0.001,0.004]$} & {$[0.001,0.007]$} & $\mathrm{B}$ \\
39 & 1.762 & {$[0.951,2.982]$} & {$[0.451,4.582]$} & $\mathrm{B}$ & 77 & 0.007 & {$[0.003,0.011]$} & {$[0.002,0.019]$} & $\mathrm{B}$ \\
40 & 0.266 & {$[0.132,0.421]$} & {$[0.068,0.710]$} & $\mathrm{B}$ & 78 & 0.002 & {$[0.001,0.004]$} & {$[0.001,0.006]$} & $\mathrm{B}$ \\
41 & 5.306 & {$[3.411,6.652]$} & {$[2.168,8.626]$} & $\mathrm{A}$ & 79 & 0.007 & {$[0.003,0.010]$} & {$[0.002,0.018]$} & $\mathrm{B}$ \\
42 & 0.635 & {$[0.319,1.019]$} & {$[0.162,1.677]$} & $\mathrm{B}$ & 80 & 0.002 & {$[0.001,0.003]$} & {$[0.001,0.006]$} & $\mathrm{B}$
\end{tabular}


Table 43. Partial ionization cross sections $\left(10^{-20} \mathrm{~m}^{2}\right)$ for 3-ethyl-2-pentene. TICS $/ 10^{-20} \mathrm{~m}^{2}=21.487 . M=98$, base peak is $\mathrm{m} / \mathrm{z} 41$.

\begin{tabular}{|c|c|c|c|c|c|c|c|c|c|}
\hline$m / z$ & PICS & $67 \%$ interval & $95 \%$ interval & unc. model & $m / z$ & PICS & $67 \%$ interval & $95 \%$ interval & unc. model \\
\hline 1 & 0.400 & {$[0.288,0.478]$} & {$[0.215,0.599]$} & $\mathrm{H}$ & 60 & 0.003 & {$[0.001,0.004]$} & {$[0.001,0.008]$} & B \\
\hline 12 & 0.010 & {$[0.005,0.015]$} & {$[0.002,0.026]$} & B & 61 & 0.014 & {$[0.007,0.022]$} & {$[0.004,0.038]$} & B \\
\hline 13 & 0.028 & {$[0.014,0.043]$} & {$[0.007,0.075]$} & B & 62 & 0.030 & {$[0.015,0.047]$} & {$[0.008,0.081]$} & B \\
\hline 14 & 0.106 & {$[0.052,0.168]$} & {$[0.027,0.284]$} & B & 63 & 0.051 & {$[0.025,0.080]$} & {$[0.013,0.139]$} & B \\
\hline 15 & 0.865 & {$[0.441,1.403]$} & {$[0.220,2.273]$} & B & 64 & 0.008 & {$[0.004,0.012]$} & {$[0.002,0.021]$} & B \\
\hline 16 & 0.033 & {$[0.016,0.051]$} & {$[0.008,0.088]$} & B & 65 & 0.072 & {$[0.035,0.112]$} & {$[0.018,0.192]$} & B \\
\hline 24 & 0.006 & {$[0.003,0.010]$} & {$[0.002,0.017]$} & B & 66 & 0.033 & {$[0.016,0.051]$} & {$[0.008,0.088]$} & B \\
\hline 25 & 0.018 & {$[0.009,0.029]$} & {$[0.005,0.050]$} & B & 67 & 0.248 & {$[0.123,0.393]$} & {$[0.064,0.664]$} & B \\
\hline 26 & 0.294 & {$[0.146,0.468]$} & {$[0.075,0.786]$} & B & 68 & 0.108 & {$[0.053,0.171]$} & {$[0.028,0.292]$} & B \\
\hline 27 & 2.239 & {$[1.249,3.858]$} & {$[0.576,5.758]$} & B & 69 & 2.181 & {$[1.207,3.729]$} & {$[0.563,5.603]$} & B \\
\hline 28 & 0.337 & {$[0.168,0.536]$} & {$[0.086,0.899]$} & B & 70 & 0.392 & {$[0.196,0.626]$} & {$[0.100,1.044]$} & B \\
\hline 29 & 1.029 & {$[0.529,1.681]$} & {$[0.262,2.702]$} & B & 71 & 0.018 & {$[0.009,0.028]$} & {$[0.005,0.048]$} & B \\
\hline 30 & 0.022 & {$[0.011,0.034]$} & {$[0.006,0.058]$} & B & 73 & 0.002 & {$[0.001,0.003]$} & {$[0.001,0.006]$} & B \\
\hline 31 & 0.011 & {$[0.005,0.016]$} & {$[0.003,0.029]$} & B & 74 & 0.004 & {$[0.002,0.006]$} & {$[0.001,0.011]$} & B \\
\hline 32 & 0.005 & {$[0.002,0.008]$} & {$[0.001,0.014]$} & B & 75 & 0.002 & {$[0.001,0.003]$} & {$[0.001,0.005]$} & B \\
\hline 36 & 0.005 & {$[0.002,0.007]$} & {$[0.001,0.013]$} & B & 76 & 0.002 & {$[0.001,0.003]$} & {$[0.000,0.005]$} & B \\
\hline 37 & 0.051 & {$[0.025,0.080]$} & {$[0.013,0.137]$} & B & 77 & 0.021 & {$[0.010,0.032]$} & {$[0.005,0.056]$} & B \\
\hline 38 & 0.154 & {$[0.076,0.242]$} & {$[0.039,0.411]$} & B & 78 & 0.005 & {$[0.003,0.008]$} & {$[0.001,0.015]$} & B \\
\hline 39 & 1.694 & {$[0.903,2.841]$} & {$[0.435,4.431]$} & B & 79 & 0.021 & {$[0.010,0.033]$} & {$[0.005,0.057]$} & B \\
\hline 40 & 0.291 & {$[0.145,0.466]$} & {$[0.075,0.781]$} & B & 80 & 0.003 & {$[0.002,0.005]$} & {$[0.001,0.009]$} & B \\
\hline 41 & 4.247 & {$[2.319,5.487]$} & {$[1.222,7.482]$} & A & 81 & 0.028 & {$[0.014,0.044]$} & {$[0.007,0.076]$} & B \\
\hline 42 & 0.433 & {$[0.216,0.691]$} & {$[0.110,1.144]$} & B & 82 & 0.006 & {$[0.003,0.010]$} & {$[0.002,0.017]$} & B \\
\hline 43 & 0.383 & {$[0.192,0.611]$} & {$[0.098,1.016]$} & B & 83 & 0.261 & {$[0.129,0.415]$} & {$[0.066,0.695]$} & B \\
\hline 44 & 0.012 & {$[0.006,0.019]$} & {$[0.003,0.032]$} & B & 84 & 0.018 & {$[0.009,0.028]$} & {$[0.005,0.047]$} & B \\
\hline 45 & 0.004 & {$[0.002,0.006]$} & {$[0.001,0.011]$} & B & 85 & 0.001 & {$[0.001,0.002]$} & {$[0.000,0.004]$} & B \\
\hline 46 & 0.004 & {$[0.002,0.006]$} & {$[0.001,0.010]$} & B & 86 & 0.001 & {$[0.001,0.002]$} & {$[0.000,0.004]$} & B \\
\hline 49 & 0.011 & {$[0.005,0.017]$} & {$[0.003,0.029]$} & B & 89 & 0.001 & {$[0.001,0.002]$} & {$[0.000,0.004]$} & B \\
\hline 50 & 0.116 & {$[0.057,0.183]$} & {$[0.030,0.312]$} & B & 91 & 0.001 & {$[0.001,0.002]$} & {$[0.000,0.004]$} & B \\
\hline 51 & 0.215 & {$[0.106,0.341]$} & {$[0.055,0.573]$} & B & 93 & 0.001 & {$[0.001,0.002]$} & {$[0.000,0.004]$} & B \\
\hline 52 & 0.091 & {$[0.045,0.144]$} & {$[0.023,0.246]$} & B & 95 & 0.001 & {$[0.001,0.002]$} & {$[0.000,0.004]$} & B \\
\hline 53 & 0.465 & {$[0.233,0.744]$} & {$[0.119,1.239]$} & B & 96 & 0.001 & {$[0.001,0.002]$} & {$[0.000,0.004]$} & B \\
\hline 54 & 0.137 & {$[0.067,0.216]$} & {$[0.035,0.365]$} & B & 97 & 0.003 & {$[0.001,0.004]$} & {$[0.001,0.008]$} & B \\
\hline 55 & 2.745 & {$[0.702,3.529]$} & {$[0.144,5.629]$} & A & 98 & 0.534 & {$[0.270,0.863]$} & {$[0.137,1.417]$} & B \\
\hline 56 & 0.827 & {$[0.423,1.348]$} & {$[0.211,2.187]$} & B & 99 & 0.042 & {$[0.020,0.065]$} & {$[0.011,0.112]$} & B \\
\hline 57 & 0.082 & {$[0.040,0.129]$} & {$[0.021,0.221]$} & B & 100 & 0.001 & {$[0.001,0.002]$} & {$[0.000,0.004]$} & B \\
\hline
\end{tabular}


Table 44. Partial ionization cross sections $\left(10^{-20} \mathrm{~m}^{2}\right)$ for 2,3,3-trimethyl-1-butene. TICS $/ 10^{-20} \mathrm{~m}^{2}=20.973 . M=98$, base peak is $\mathrm{m} / \mathrm{z}$ 83.

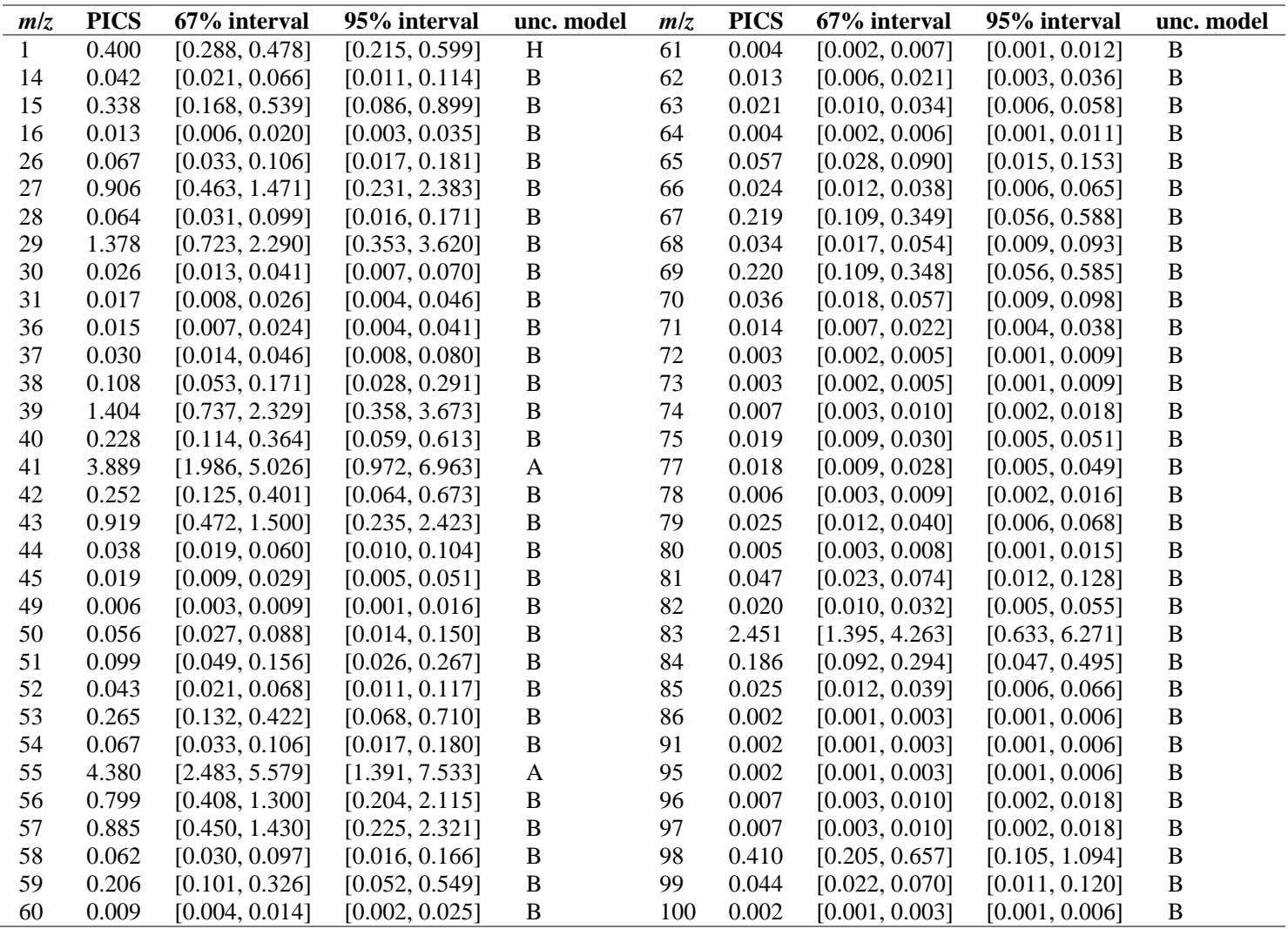


Table 45. Partial ionization cross sections $\left(10^{-20} \mathrm{~m}^{2}\right)$ for 2-ethyl-3-methyl-1-butene. TICS $/ 10^{-20} \mathrm{~m}^{2}=21.226 . M=98$, base peak is $\mathrm{m} / \mathrm{z}$ 41.

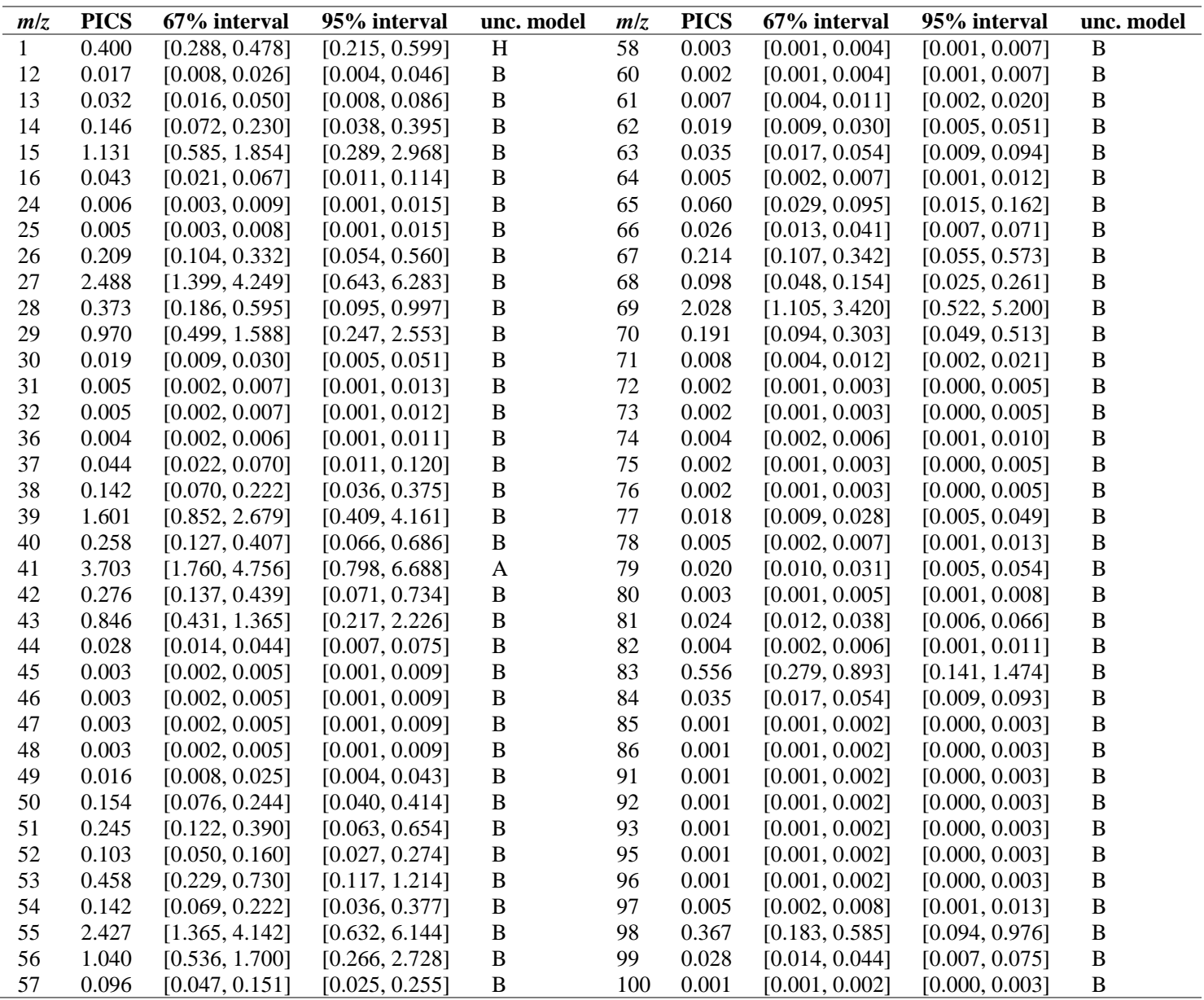


Table 46. Partial ionization cross sections $\left(10^{-20} \mathrm{~m}^{2}\right)$ for benzaldehyde. TICS $/ 10^{-20} \mathrm{~m}^{2}=17.773 . M=106$, base peak is $\mathrm{m} / \mathrm{z} 77$.

\begin{tabular}{|c|c|c|c|c|c|c|c|c|c|}
\hline$m / z$ & PICS & $67 \%$ interval & $95 \%$ interval & unc. model & $m / z$ & PICS & $67 \%$ interval & $95 \%$ interval & unc. model \\
\hline 1 & 0.400 & {$[0.288,0.478]$} & {$[0.215,0.599]$} & $\mathrm{H}$ & 53 & 0.143 & {$[0.070,0.225]$} & {$[0.036,0.380]$} & B \\
\hline 17 & 0.022 & {$[0.011,0.035]$} & {$[0.006,0.060]$} & B & 60 & 0.037 & {$[0.018,0.058]$} & {$[0.009,0.099]$} & B \\
\hline 18 & 0.046 & {$[0.023,0.072]$} & {$[0.012,0.124]$} & B & 61 & 0.089 & {$[0.044,0.141]$} & {$[0.023,0.241]$} & B \\
\hline 25 & 0.049 & {$[0.024,0.077]$} & {$[0.013,0.132]$} & B & 62 & 0.120 & {$[0.059,0.189]$} & {$[0.031,0.321]$} & B \\
\hline 26 & 0.217 & {$[0.107,0.344]$} & {$[0.056,0.580]$} & B & 63 & 0.139 & {$[0.068,0.220]$} & {$[0.036,0.372]$} & B \\
\hline 27 & 0.400 & {$[0.200,0.640]$} & {$[0.102,1.067]$} & B & 73 & 0.110 & {$[0.054,0.174]$} & {$[0.028,0.295]$} & B \\
\hline 28 & 0.071 & {$[0.035,0.112]$} & {$[0.018,0.191]$} & B & 74 & 0.350 & {$[0.175,0.561]$} & {$[0.089,0.929]$} & B \\
\hline 29 & 0.450 & {$[0.225,0.719]$} & {$[0.116,1.200]$} & B & 75 & 0.154 & {$[0.076,0.244]$} & {$[0.039,0.413]$} & B \\
\hline 36 & 0.046 & {$[0.023,0.073]$} & {$[0.012,0.124]$} & B & 76 & 0.026 & {$[0.013,0.041]$} & {$[0.007,0.071]$} & B \\
\hline 37 & 0.364 & {$[0.182,0.582]$} & {$[0.092,0.967]$} & B & 77 & 3.342 & {$[1.724,4.303]$} & {$[0.884,5.982]$} & A \\
\hline 38 & 0.444 & {$[0.223,0.711]$} & {$[0.113,1.174]$} & B & 78 & 0.536 & {$[0.272,0.867]$} & {$[0.137,1.427]$} & B \\
\hline 39 & 0.525 & {$[0.264,0.843]$} & {$[0.134,1.394]$} & B & 79 & 0.034 & {$[0.017,0.054]$} & {$[0.009,0.092]$} & B \\
\hline 40 & 0.035 & {$[0.017,0.055]$} & {$[0.009,0.094]$} & B & 85 & 0.013 & {$[0.006,0.020]$} & {$[0.003,0.035]$} & B \\
\hline 43 & 0.036 & {$[0.018,0.057]$} & {$[0.009,0.098]$} & B & 86 & 0.016 & {$[0.008,0.025]$} & {$[0.004,0.044]$} & B \\
\hline 49 & 0.273 & {$[0.135,0.434]$} & {$[0.069,0.727]$} & B & 105 & 1.873 & [1.032, 3.190] & {$[0.480,4.798]$} & B \\
\hline 50 & 1.709 & {$[0.935,2.912]$} & {$[0.437,4.411]$} & B & 106 & 1.969 & {$[1.098,3.380]$} & {$[0.508,5.063]$} & B \\
\hline 51 & 2.802 & {$[1.171,3.651]$} & {$[0.467,5.331]$} & A & 107 & 0.160 & {$[0.079,0.252]$} & {$[0.041,0.426]$} & B \\
\hline 52 & 0.772 & {$[0.394,1.252]$} & {$[0.198,2.042]$} & B & & & & & \\
\hline
\end{tabular}

Table 47. Partial ionization cross sections $\left(10^{-20} \mathrm{~m}^{2}\right)$ for phenol. TICS $/ 10^{-20} \mathrm{~m}^{2}=16.457 . M=94$ is base peak.

\begin{tabular}{|c|c|c|c|c|c|c|c|c|c|}
\hline$m / z$ & PICS & $67 \%$ interval & $95 \%$ interval & unc. model & $m / z$ & PICS & $67 \%$ interval & $\mathbf{9 5 \%}$ interval & unc. model \\
\hline 1 & 0.400 & {$[0.287,0.477]$} & {$[0.215,0.599]$} & $\mathrm{H}$ & 51 & 0.359 & {$[0.181,0.578]$} & {$[0.092,0.951]$} & B \\
\hline 12 & 0.022 & {$[0.011,0.034]$} & {$[0.006,0.059]$} & B & 52 & 0.065 & {$[0.032,0.102]$} & {$[0.017,0.175]$} & B \\
\hline 13 & 0.028 & {$[0.014,0.044]$} & {$[0.007,0.076]$} & B & 53 & 0.234 & {$[0.116,0.372]$} & {$[0.060,0.627]$} & B \\
\hline 14 & 0.018 & {$[0.009,0.028]$} & {$[0.005,0.049]$} & B & 54 & 0.007 & {$[0.003,0.011]$} & {$[0.002,0.019]$} & B \\
\hline 15 & 0.074 & {$[0.036,0.116]$} & {$[0.019,0.199]$} & B & 55 & 0.506 & {$[0.254,0.813]$} & {$[0.129,1.343]$} & B \\
\hline 17 & 0.006 & {$[0.003,0.010]$} & {$[0.002,0.017]$} & B & 56 & 0.019 & {$[0.009,0.030]$} & {$[0.005,0.052]$} & B \\
\hline 19 & 0.021 & {$[0.010,0.033]$} & {$[0.005,0.056]$} & B & 60 & 0.039 & {$[0.019,0.062]$} & {$[0.010,0.106]$} & B \\
\hline 24 & 0.013 & {$[0.006,0.020]$} & {$[0.003,0.035]$} & B & 61 & 0.159 & {$[0.079,0.254]$} & {$[0.041,0.428]$} & B \\
\hline 25 & 0.078 & {$[0.038,0.123]$} & {$[0.020,0.209]$} & B & 62 & 0.234 & {$[0.117,0.374]$} & {$[0.060,0.625]$} & B \\
\hline 26 & 0.337 & {$[0.168,0.538]$} & {$[0.086,0.901]$} & B & 63 & 0.378 & {$[0.188,0.602]$} & {$[0.096,1.004]$} & B \\
\hline 27 & 0.435 & {$[0.219,0.697]$} & {$[0.112,1.157]$} & B & 64 & 0.141 & {$[0.070,0.223]$} & {$[0.036,0.378]$} & B \\
\hline 29 & 0.276 & {$[0.137,0.438]$} & {$[0.071,0.735]$} & B & 65 & 1.338 & {$[0.720,2.262]$} & {$[0.343,3.496]$} & B \\
\hline 30 & 0.012 & {$[0.006,0.019]$} & {$[0.003,0.032]$} & B & 66 & 1.898 & {$[1.077,3.291]$} & {$[0.491,4.859]$} & B \\
\hline 31 & 0.070 & {$[0.034,0.110]$} & {$[0.018,0.187]$} & B & 67 & 0.130 & {$[0.064,0.207]$} & {$[0.034,0.351]$} & B \\
\hline 36 & 0.065 & {$[0.032,0.102]$} & {$[0.017,0.175]$} & B & 68 & 0.051 & {$[0.025,0.080]$} & {$[0.013,0.136]$} & B \\
\hline 37 & 0.491 & {$[0.248,0.791]$} & {$[0.125,1.298]$} & B & 72 & 0.005 & {$[0.002,0.007]$} & {$[0.001,0.012]$} & B \\
\hline 38 & 0.849 & {$[0.438,1.393]$} & {$[0.215,2.229]$} & B & 73 & 0.038 & {$[0.019,0.060]$} & {$[0.010,0.102]$} & B \\
\hline 39 & 2.176 & {$[0.632,2.825]$} & {$[0.147,4.388]$} & A & 74 & 0.084 & {$[0.041,0.133]$} & {$[0.021,0.225]$} & B \\
\hline 40 & 1.005 & {$[0.526,1.665]$} & {$[0.257,2.643]$} & B & 75 & 0.040 & {$[0.019,0.062]$} & {$[0.010,0.108]$} & B \\
\hline 41 & 0.082 & {$[0.040,0.130]$} & {$[0.021,0.222]$} & B & 76 & 0.028 & {$[0.014,0.044]$} & {$[0.007,0.076]$} & B \\
\hline 42 & 0.098 & {$[0.048,0.155]$} & {$[0.025,0.262]$} & B & 77 & 0.029 & {$[0.014,0.046]$} & {$[0.008,0.079]$} & B \\
\hline 43 & 0.052 & {$[0.025,0.081]$} & {$[0.013,0.140]$} & B & 79 & 0.035 & {$[0.017,0.054]$} & {$[0.009,0.094]$} & B \\
\hline 45 & 0.009 & {$[0.004,0.013]$} & {$[0.002,0.023]$} & B & 91 & 0.003 & {$[0.001,0.005]$} & {$[0.001,0.008]$} & B \\
\hline 46 & 0.067 & {$[0.033,0.106]$} & {$[0.017,0.179]$} & B & 94 & 2.910 & {$[1.421,3.788]$} & {$[0.676,5.320]$} & A \\
\hline 47 & 0.286 & {$[0.143,0.455]$} & {$[0.073,0.758]$} & B & 95 & 0.198 & {$[0.098,0.315]$} & {$[0.051,0.530]$} & B \\
\hline 49 & 0.101 & {$[0.049,0.158]$} & {$[0.026,0.271]$} & B & 96 & 0.011 & {$[0.005,0.017]$} & {$[0.003,0.030]$} & B \\
\hline 50 & 0.446 & {$[0.224,0.716]$} & {$[0.114,1.181]$} & B & & & & & \\
\hline
\end{tabular}


Table 48. Partial ionization cross sections $\left(10^{-20} \mathrm{~m}^{2}\right)$ for acetophenone. TICS $/ 10^{-20} \mathrm{~m}^{2}=21 \cdot 152 . M=120$, base peak is $m / z 105$.

\begin{tabular}{|c|c|c|c|c|c|c|c|c|c|}
\hline$m / z$ & PICS & $67 \%$ interval & $95 \%$ interval & unc. model & $m / z$ & PICS & $67 \%$ interval & $95 \%$ interval & unc. model \\
\hline 1 & 0.400 & {$[0.288,0.478]$} & {$[0.215,0.600]$} & $\mathrm{H}$ & 65 & 0.162 & {$[0.080,0.257]$} & {$[0.041,0.433]$} & B \\
\hline 26 & 0.139 & {$[0.069,0.221]$} & {$[0.036,0.375]$} & B & 66 & 0.013 & {$[0.006,0.021]$} & {$[0.003,0.036]$} & B \\
\hline 27 & 0.504 & {$[0.253,0.806]$} & {$[0.128,1.326]$} & B & 72 & 0.006 & {$[0.003,0.009]$} & {$[0.002,0.016]$} & B \\
\hline 28 & 0.107 & {$[0.052,0.167]$} & {$[0.027,0.286]$} & B & 73 & 0.070 & {$[0.035,0.111]$} & {$[0.018,0.190]$} & B \\
\hline 29 & 0.052 & {$[0.025,0.081]$} & {$[0.013,0.140]$} & B & 74 & 0.313 & {$[0.156,0.498]$} & {$[0.080,0.836]$} & B \\
\hline 36 & 0.011 & {$[0.005,0.018]$} & {$[0.003,0.030]$} & B & 75 & 0.165 & {$[0.081,0.261]$} & {$[0.042,0.442]$} & B \\
\hline 37 & 0.219 & {$[0.108,0.346]$} & {$[0.056,0.587]$} & B & 76 & 0.257 & {$[0.127,0.408]$} & {$[0.066,0.688]$} & B \\
\hline 38 & 0.496 & {$[0.249,0.796]$} & {$[0.127,1.314]$} & B & 77 & 4.775 & {$[2.870,6.093]$} & {$[1.704,8.107]$} & A \\
\hline 39 & 0.784 & {$[0.398,1.270]$} & {$[0.199,2.066]$} & B & 78 & 0.453 & {$[0.226,0.725]$} & {$[0.116,1.208]$} & B \\
\hline 40 & 0.042 & {$[0.020,0.066]$} & {$[0.011,0.113]$} & B & 79 & 0.016 & {$[0.008,0.025]$} & {$[0.004,0.043]$} & B \\
\hline 41 & 0.072 & {$[0.035,0.112]$} & {$[0.018,0.193]$} & B & 85 & 0.009 & {$[0.005,0.015]$} & {$[0.002,0.026]$} & B \\
\hline 42 & 0.200 & {$[0.098,0.314]$} & {$[0.051,0.533]$} & B & 86 & 0.014 & {$[0.007,0.022]$} & {$[0.004,0.037]$} & B \\
\hline 43 & 1.772 & {$[0.946,2.982]$} & {$[0.449,4.604]$} & B & 87 & 0.009 & {$[0.004,0.014]$} & {$[0.002,0.024]$} & B \\
\hline 44 & 0.048 & {$[0.024,0.076]$} & {$[0.012,0.129]$} & B & 89 & 0.043 & {$[0.021,0.067]$} & {$[0.011,0.116]$} & B \\
\hline 45 & 0.019 & {$[0.009,0.030]$} & {$[0.005,0.051]$} & B & 90 & 0.008 & {$[0.004,0.013]$} & {$[0.002,0.023]$} & B \\
\hline 48 & 0.009 & {$[0.004,0.014]$} & {$[0.002,0.024]$} & B & 91 & 0.074 & {$[0.036,0.117]$} & {$[0.019,0.199]$} & B \\
\hline 49 & 0.150 & {$[0.074,0.238]$} & {$[0.038,0.401]$} & B & 92 & 0.004 & {$[0.002,0.006]$} & {$[0.001,0.011]$} & B \\
\hline 50 & 1.471 & {$[0.768,2.432]$} & {$[0.374,3.847]$} & B & 98 & 0.003 & {$[0.002,0.005]$} & {$[0.001,0.009]$} & B \\
\hline 51 & 3.244 & {$[1.289,4.288]$} & {$[0.465,6.317]$} & A & 102 & 0.003 & {$[0.001,0.005]$} & {$[0.001,0.008]$} & B \\
\hline 52 & 0.344 & {$[0.171,0.548]$} & {$[0.088,0.914]$} & B & 103 & 0.006 & {$[0.003,0.009]$} & {$[0.002,0.016]$} & B \\
\hline 53 & 0.123 & {$[0.061,0.197]$} & {$[0.032,0.334]$} & B & 105 & 2.845 & {$[0.839,3.732]$} & {$[0.207,5.822]$} & A \\
\hline 60 & 0.015 & {$[0.007,0.023]$} & {$[0.004,0.039]$} & B & 106 & 0.207 & {$[0.102,0.328]$} & {$[0.053,0.555]$} & B \\
\hline 61 & 0.093 & {$[0.046,0.148]$} & {$[0.024,0.253]$} & B & 107 & 0.011 & {$[0.005,0.018]$} & {$[0.003,0.031]$} & B \\
\hline 62 & 0.163 & {$[0.080,0.258]$} & {$[0.042,0.437]$} & B & 120 & 0.868 & {$[0.442,1.408]$} & {$[0.221,2.288]$} & B \\
\hline 63 & 0.223 & {$[0.110,0.354]$} & {$[0.057,0.598]$} & B & 121 & 0.071 & {$[0.035,0.112]$} & {$[0.018,0.190]$} & B \\
\hline 64 & 0.041 & {$[0.020,0.065]$} & {$[0.011,0.112]$} & B & 122 & 0.003 & {$[0.001,0.004]$} & {$[0.001,0.008]$} & B \\
\hline
\end{tabular}

Table 49. Partial ionization cross sections $\left(10^{-20} \mathrm{~m}^{2}\right)$ for pyrrole. TICS $/ 10^{-20} \mathrm{~m}^{2}=12.426 . M=67$ is base peak.

\begin{tabular}{|c|c|c|c|c|c|c|c|c|c|}
\hline$m / z$ & PICS & $67 \%$ interval & $95 \%$ interval & unc. model & $m / z$ & PICS & $67 \%$ interval & $95 \%$ interval & unc. model \\
\hline 1 & 0.400 & {$[0.288,0.478]$} & {$[0.216,0.601]$} & $\mathrm{H}$ & 38 & 0.790 & {$[0.410,1.296]$} & {$[0.202,2.065]$} & B \\
\hline 2 & 0.023 & {$[0.011,0.035]$} & {$[0.006,0.061]$} & B & 39 & 2.077 & {$[0.941,2.717]$} & {$[0.412,3.924]$} & A \\
\hline 12 & 0.122 & {$[0.061,0.194]$} & {$[0.032,0.329]$} & B & 40 & 1.598 & {$[0.419,2.082]$} & {$[0.096,3.362]$} & A \\
\hline 13 & 0.127 & {$[0.063,0.202]$} & {$[0.033,0.341]$} & B & 41 & 1.814 & {$[0.661,2.391]$} & {$[0.227,3.629]$} & A \\
\hline 14 & 0.142 & {$[0.070,0.224]$} & {$[0.036,0.379]$} & B & 42 & 0.044 & {$[0.022,0.070]$} & {$[0.011,0.120]$} & B \\
\hline 15 & 0.032 & {$[0.016,0.050]$} & {$[0.008,0.086]$} & B & 48 & 0.004 & {$[0.002,0.006]$} & {$[0.001,0.011]$} & B \\
\hline 16 & 0.003 & {$[0.001,0.004]$} & {$[0.001,0.007]$} & B & 49 & 0.014 & {$[0.007,0.021]$} & {$[0.003,0.037]$} & B \\
\hline 24 & 0.036 & {$[0.017,0.056]$} & {$[0.009,0.097]$} & B & 50 & 0.023 & {$[0.011,0.036]$} & {$[0.006,0.061]$} & B \\
\hline 25 & 0.113 & {$[0.056,0.181]$} & {$[0.029,0.305]$} & B & 51 & 0.024 & {$[0.012,0.038]$} & {$[0.006,0.066]$} & B \\
\hline 26 & 0.258 & {$[0.129,0.413]$} & {$[0.066,0.688]$} & B & 52 & 0.051 & {$[0.025,0.080]$} & {$[0.013,0.137]$} & B \\
\hline 27 & 0.199 & {$[0.099,0.315]$} & {$[0.051,0.532]$} & B & 53 & 0.002 & {$[0.001,0.003]$} & {$[0.000,0.005]$} & B \\
\hline 28 & 2.172 & {$[1.037,2.826]$} & {$[0.493,4.038]$} & A & 62 & 0.010 & {$[0.005,0.015]$} & {$[0.002,0.026]$} & B \\
\hline 29 & 0.032 & {$[0.016,0.051]$} & {$[0.008,0.087]$} & B & 63 & 0.016 & {$[0.008,0.024]$} & {$[0.004,0.042]$} & B \\
\hline 30 & 0.005 & {$[0.002,0.008]$} & {$[0.001,0.013]$} & B & 64 & 0.016 & {$[0.008,0.026]$} & {$[0.004,0.044]$} & B \\
\hline 31 & 0.001 & {$[0.000,0.001]$} & {$[0.000,0.002]$} & B & 65 & 0.013 & {$[0.006,0.020]$} & {$[0.003,0.035]$} & B \\
\hline 32 & 0.023 & {$[0.011,0.037]$} & {$[0.006,0.063]$} & B & 66 & 0.110 & {$[0.054,0.174]$} & {$[0.028,0.295]$} & B \\
\hline 33 & 0.009 & {$[0.004,0.014]$} & {$[0.002,0.024]$} & B & 67 & 1.397 & {$[0.758,2.357]$} & {$[0.355,3.575]$} & B \\
\hline 34 & 0.012 & {$[0.006,0.019]$} & {$[0.003,0.033]$} & B & 68 & 0.066 & {$[0.033,0.105]$} & {$[0.017,0.177]$} & B \\
\hline 36 & 0.096 & {$[0.047,0.152]$} & {$[0.025,0.259]$} & B & 69 & 0.001 & {$[0.000,0.001]$} & {$[0.000,0.002]$} & B \\
\hline 37 & 0.553 & {$[0.284,0.902]$} & {$[0.141,1.450]$} & B & & & & & \\
\hline
\end{tabular}


Table 50. Partial ionization cross sections $\left(10^{-20} \mathrm{~m}^{2}\right)$ for pyridine. TICS $/ 10^{-20} \mathrm{~m}^{2}=13.915 . M=79$ is base peak.

\begin{tabular}{|c|c|c|c|c|c|c|c|c|c|}
\hline $\mathbf{m} / \mathbf{z}$ & PICS & $67 \%$ interval & $95 \%$ interval & unc. model & $m / z$ & PICS & $67 \%$ interval & $95 \%$ interval & unc. model \\
\hline 1 & 0.400 & {$[0.289,0.479]$} & {$[0.215,0.600]$} & $\mathrm{H}$ & 52 & 3.877 & {$[2.613,4.772]$} & {$[1.761,6.092]$} & A \\
\hline 25 & 0.056 & {$[0.028,0.089]$} & {$[0.015,0.153]$} & B & 53 & 0.410 & {$[0.208,0.664]$} & {$[0.104,1.083]$} & B \\
\hline 26 & 0.643 & {$[0.330,1.050]$} & {$[0.164,1.694]$} & B & 54 & 0.013 & {$[0.006,0.021]$} & {$[0.003,0.035]$} & B \\
\hline 27 & 0.185 & {$[0.092,0.294]$} & {$[0.047,0.495]$} & B & 62 & 0.005 & {$[0.002,0.008]$} & {$[0.001,0.014]$} & B \\
\hline 28 & 0.052 & {$[0.025,0.082]$} & {$[0.013,0.141]$} & B & 63 & 0.005 & {$[0.002,0.008]$} & {$[0.001,0.013]$} & B \\
\hline 36 & 0.011 & {$[0.005,0.017]$} & {$[0.003,0.028]$} & B & 64 & 0.014 & {$[0.007,0.022]$} & {$[0.004,0.038]$} & B \\
\hline 37 & 0.142 & {$[0.071,0.227]$} & {$[0.036,0.381]$} & B & 74 & 0.007 & {$[0.004,0.012]$} & {$[0.002,0.020]$} & B \\
\hline 38 & 0.179 & {$[0.089,0.284]$} & {$[0.046,0.479]$} & B & 75 & 0.059 & {$[0.029,0.093]$} & {$[0.015,0.158]$} & B \\
\hline 39 & 0.583 & {$[0.298,0.948]$} & {$[0.148,1.530]$} & B & 76 & 0.044 & {$[0.022,0.070]$} & {$[0.011,0.119]$} & B \\
\hline 40 & 0.047 & {$[0.023,0.074]$} & {$[0.012,0.127]$} & B & 77 & 0.011 & {$[0.005,0.017]$} & {$[0.003,0.030]$} & B \\
\hline 48 & 0.023 & {$[0.011,0.036]$} & {$[0.006,0.062]$} & B & 78 & 0.419 & {$[0.211,0.674]$} & {$[0.106,1.105]$} & B \\
\hline 49 & 0.195 & {$[0.096,0.309]$} & {$[0.050,0.518]$} & B & 79 & 3.714 & {$[2.460,4.601]$} & {$[1.618,5.907]$} & A \\
\hline 50 & 0.995 & {$[0.526,1.659]$} & {$[0.252,2.582]$} & B & 80 & 0.234 & {$[0.116,0.371]$} & {$[0.060,0.623]$} & B \\
\hline 51 & 1.587 & {$[0.893,2.734]$} & {$[0.406,4.039]$} & B & 81 & 0.004 & {$[0.002,0.006]$} & {$[0.001,0.010]$} & B \\
\hline
\end{tabular}

Table 51. Partial ionization cross sections $\left(10^{-20} \mathrm{~m}^{2}\right)$ for benzonitrile. TICS $/ 10^{-20} \mathrm{~m}^{2}=16.783 . M=103$ is base peak.

\begin{tabular}{|c|c|c|c|c|c|c|c|c|c|}
\hline$m / z$ & PICS & $67 \%$ interval & $95 \%$ interval & unc. model & $m / \mathbf{z}$ & PICS & $67 \%$ interval & $95 \%$ interval & unc. mode \\
\hline 1 & 0.400 & {$[0.289,0.479]$} & {$[0.215,0.599]$} & $\mathrm{H}$ & 72 & 0.015 & {$[0.007,0.024]$} & {$[0.004,0.041]$} & B \\
\hline 25 & 0.020 & {$[0.010,0.032]$} & {$[0.005,0.055]$} & B & 73 & 0.118 & {$[0.059,0.189]$} & {$[0.030,0.319]$} & B \\
\hline 26 & 0.238 & {$[0.119,0.380]$} & {$[0.061,0.635]$} & B & 74 & 0.354 & {$[0.177,0.567]$} & {$[0.090,0.938]$} & B \\
\hline 27 & 0.174 & {$[0.087,0.278]$} & {$[0.045,0.468]$} & B & 75 & 0.691 & {$[0.352,1.122]$} & {$[0.175,1.812]$} & B \\
\hline 36 & 0.047 & {$[0.023,0.074]$} & {$[0.012,0.127]$} & B & 76 & 2.571 & {$[1.030,3.407]$} & {$[0.374,5.004]$} & A \\
\hline 37 & 0.416 & {$[0.210,0.671]$} & {$[0.106,1.103]$} & B & 77 & 0.395 & {$[0.197,0.631]$} & {$[0.100,1.044]$} & B \\
\hline 38 & 0.392 & {$[0.198,0.631]$} & {$[0.101,1.043]$} & B & 78 & 0.020 & {$[0.009,0.030]$} & {$[0.005,0.053]$} & B \\
\hline 39 & 0.591 & {$[0.299,0.952]$} & {$[0.151,1.566]$} & B & 84 & 0.005 & {$[0.003,0.009]$} & {$[0.001,0.015]$} & B \\
\hline 40 & 0.043 & {$[0.021,0.068]$} & {$[0.011,0.117]$} & B & 85 & 0.011 & {$[0.005,0.017]$} & {$[0.003,0.029]$} & B \\
\hline 43 & 0.014 & {$[0.007,0.021]$} & {$[0.003,0.037]$} & B & 86 & 0.015 & {$[0.007,0.024]$} & {$[0.004,0.042]$} & B \\
\hline 48 & 0.049 & {$[0.024,0.078]$} & {$[0.013,0.134]$} & B & 87 & 0.015 & {$[0.007,0.023]$} & {$[0.004,0.040]$} & B \\
\hline 49 & 0.327 & {$[0.163,0.523]$} & {$[0.084,0.875]$} & B & 88 & 0.033 & {$[0.016,0.052]$} & {$[0.009,0.090]$} & B \\
\hline 50 & 2.031 & {$[0.424,2.637]$} & {$[0.052,4.321]$} & A & 89 & 0.005 & {$[0.002,0.007]$} & {$[0.001,0.012]$} & B \\
\hline 51 & 1.200 & {$[0.634,2.009]$} & {$[0.304,3.130]$} & B & 98 & 0.009 & {$[0.004,0.014]$} & {$[0.002,0.024]$} & B \\
\hline 52 & 0.628 & {$[0.320,1.020]$} & {$[0.161,1.670]$} & B & 99 & 0.058 & {$[0.028,0.091]$} & {$[0.015,0.156]$} & B \\
\hline 53 & 0.078 & {$[0.038,0.123]$} & {$[0.020,0.211]$} & B & 100 & 0.022 & {$[0.011,0.035]$} & {$[0.006,0.060]$} & B \\
\hline 60 & 0.039 & {$[0.019,0.061]$} & {$[0.010,0.105]$} & B & 101 & 0.013 & {$[0.006,0.021]$} & {$[0.003,0.036]$} & B \\
\hline 61 & 0.144 & {$[0.070,0.226]$} & {$[0.037,0.385]$} & B & 102 & 0.085 & {$[0.042,0.134]$} & {$[0.022,0.229]$} & B \\
\hline 62 & 0.150 & {$[0.074,0.238]$} & {$[0.038,0.401]$} & B & 103 & 4.458 & {$[2.941,5.581]$} & {$[1.924,7.191]$} & A \\
\hline 63 & 0.312 & {$[0.157,0.502]$} & {$[0.080,0.833]$} & B & 104 & 0.401 & {$[0.200,0.641]$} & {$[0.102,1.066]$} & B \\
\hline 64 & 0.162 & {$[0.080,0.258]$} & {$[0.042,0.435]$} & B & 105 & 0.013 & {$[0.007,0.021]$} & {$[0.003,0.036]$} & B \\
\hline 65 & 0.018 & {$[0.009,0.028]$} & {$[0.005,0.048]$} & B & & & & & \\
\hline
\end{tabular}




\section{Appendix}

Table A1. Molecular orbital data for thiophenol. Double-ionization threshold $=22.50 \mathrm{eV}$ from CCSD(T)/cc-pV(T+d)Z (to singlet dication).

\begin{tabular}{|c|c|c|c|c|c|}
\hline MO & $\mathrm{B} / \mathrm{eV}$ & $\mathrm{U} / \mathrm{eV}$ & $\mathbf{N}$ & DblIon & Remarks \\
\hline 1 & 2502.6 & 3297.09 & 2 & Yes & \\
\hline 2 & 307.01 & 436.08 & 2 & Yes & \\
\hline 3 & 306.03 & 435.81 & 2 & Yes & \\
\hline 4 & 306.0 & 435.87 & 2 & Yes & \\
\hline 5 & 305.99 & 436.15 & 2 & Yes & \\
\hline 6 & 305.94 & 436.08 & 2 & Yes & \\
\hline 7 & 305.82 & 436.04 & 2 & Yes & \\
\hline 8 & 244.12 & 509.17 & 2 & Yes & \\
\hline 9 & 181.05 & 477.93 & 2 & Yes & \\
\hline 10 & 181.02 & 478.6 & 2 & Yes & \\
\hline 11 & 180.97 & 479.58 & 2 & Yes & \\
\hline 12 & 31.77 & 38.1 & 2 & Yes & \\
\hline 13 & 28.81 & 32.04 & 2 & Yes & \\
\hline 14 & 27.93 & 42.13 & 2 & Yes & B from P3 \\
\hline 15 & 25.78 & 27.65 & 2 & Yes & B from P3 \\
\hline 16 & 18.99 & 38.3 & 2 & No & B from P2 \\
\hline 17 & 22.08 & 37.18 & 2 & No & \\
\hline 18 & 17.11 & 26.2 & 2 & No & B from P3 \\
\hline 19 & 15.75 & 33.87 & 2 & No & B from P3 \\
\hline 20 & 15.43 & 36.34 & 2 & No & B from P3 \\
\hline 21 & 14.6 & 32.08 & 2 & No & B from P3 \\
\hline 22 & 14.31 & 30.9 & 2 & No & B from P3 \\
\hline 23 & 13.02 & 30.1 & 2 & No & B from P3 \\
\hline 24 & 12.69 & 23.11 & 2 & No & B from P3 \\
\hline 25 & 12.22 & 37.18 & 2 & No & B from expt \\
\hline 26 & 11.61 & 28.65 & 2 & No & B from expt \\
\hline 27 & 10.61 & 21.39 & 2 & No & B from expt \\
\hline 28 & 9.41 & 28.19 & 2 & No & B from expt \\
\hline 29 & 8.49 & 26.99 & 2 & No & B from expt \\
\hline
\end{tabular}


Table A2. Molecular orbital data for $o$-xylene. Double-ionization threshold $=23.14 \mathrm{eV}$ from CCSD(T)/cc-pV(T+d)Z (to triplet dication).

\begin{tabular}{|c|c|c|c|c|c|}
\hline MO & $\mathrm{B} / \mathrm{eV}$ & U/eV & $\mathbf{N}$ & DblIon & Remarks \\
\hline 1 & 305.78 & 435.83 & 2 & Yes & \\
\hline 2 & 305.74 & 436.16 & 2 & Yes & \\
\hline 3 & 305.53 & 435.71 & 2 & Yes & \\
\hline 4 & 305.51 & 435.9 & 2 & Yes & \\
\hline 5 & 305.49 & 436.1 & 2 & Yes & \\
\hline 6 & 305.47 & 436.27 & 2 & Yes & \\
\hline 7 & 305.43 & 436.01 & 2 & Yes & \\
\hline 8 & 305.43 & 436.01 & 2 & Yes & \\
\hline 9 & 31.39 & 38.85 & 2 & Yes & B from P3 \\
\hline 10 & 28.54 & 39.32 & 2 & Yes & B from P3 \\
\hline 11 & 28.07 & 39.92 & 2 & Yes & \\
\hline 12 & 25.75 & 38.92 & 2 & Yes & B from P3 \\
\hline 13 & 20.96 & 38.0 & 2 & No & B from $\mathrm{P} 2$ \\
\hline 14 & 18.35 & 37.27 & 2 & No & B from $\mathrm{P} 2$ \\
\hline 15 & 19.23 & 39.16 & 2 & No & B from P3 \\
\hline 16 & 16.44 & 27.52 & 2 & No & B from P3 \\
\hline 17 & 15.61 & 33.88 & 2 & No & B from P3 \\
\hline 18 & 15.05 & 34.9 & 2 & No & B from P3 \\
\hline 19 & 14.6 & 23.53 & 2 & No & B from P3 \\
\hline 20 & 13.97 & 32.63 & 2 & No & B from P3 \\
\hline 21 & 13.62 & 34.84 & 2 & No & B from P3 \\
\hline 22 & 13.75 & 26.56 & 2 & No & B from P3 \\
\hline 23 & 13.4 & 29.58 & 2 & No & B from P3 \\
\hline 24 & 13.27 & 32.69 & 2 & No & B from P3 \\
\hline 25 & 11.74 & 38.11 & 2 & No & B from P3 \\
\hline 26 & 11.7 & 24.91 & 2 & No & B from P3 \\
\hline 27 & 11.68 & 39.96 & 2 & No & B from P3 \\
\hline 28 & 8.88 & 28.49 & 2 & No & B from P3 \\
\hline 29 & 8.56 & 29.4 & 2 & No & B from expt \\
\hline
\end{tabular}


Table A3. Molecular orbital data for $m$-xylene. Double-ionization threshold $=23.11 \mathrm{eV}$ from CCSD(T)/cc-pV(T+d)Z (to triplet dication).

\begin{tabular}{|c|c|c|c|c|c|}
\hline MO & $\mathrm{B} / \mathrm{eV}$ & U/eV & $\mathbf{N}$ & DblIon & Remarks \\
\hline 1 & 305.83 & 435.99 & 2 & Yes & \\
\hline 2 & 305.83 & 436.01 & 2 & Yes & \\
\hline 3 & 305.61 & 435.94 & 2 & Yes & \\
\hline 4 & 305.42 & 436.01 & 2 & Yes & \\
\hline 5 & 305.42 & 436.01 & 2 & Yes & \\
\hline 6 & 305.39 & 435.99 & 2 & Yes & \\
\hline 7 & 305.39 & 436.04 & 2 & Yes & \\
\hline 8 & 305.38 & 436.01 & 2 & Yes & \\
\hline 9 & 31.37 & 38.96 & 2 & Yes & \\
\hline 10 & 28.68 & 39.17 & 2 & Yes & \\
\hline 11 & 27.92 & 40.35 & 2 & Yes & \\
\hline 12 & 25.52 & 37.34 & 2 & Yes & B from P3 \\
\hline 13 & 25.16 & 39.23 & 2 & Yes & \\
\hline 14 & 18.39 & 37.64 & 2 & No & B from $\mathrm{P} 2$ \\
\hline 15 & 18.59 & 39.19 & 2 & No & B from P3 \\
\hline 16 & 16.5 & 27.33 & 2 & No & B from P3 \\
\hline 17 & 15.53 & 34.53 & 2 & No & B from P3 \\
\hline 18 & 14.98 & 34.56 & 2 & No & B from P3 \\
\hline 19 & 14.28 & 24.31 & 2 & No & B from P3 \\
\hline 20 & 13.89 & 33.32 & 2 & No & B from P3 \\
\hline 21 & 14.16 & 25.43 & 2 & No & B from P3 \\
\hline 22 & 13.63 & 34.3 & 2 & No & B from P3 \\
\hline 23 & 13.68 & 28.77 & 2 & No & B from P3 \\
\hline 24 & 13.0 & 32.43 & 2 & No & B from P3 \\
\hline 25 & 11.73 & 39.84 & 2 & No & B from P3 \\
\hline 26 & 11.73 & 38.14 & 2 & No & B from P3 \\
\hline 27 & 11.6 & 25.61 & 2 & No & B from P3 \\
\hline 28 & 8.86 & 28.55 & 2 & No & B from P3 \\
\hline 29 & 8.56 & 29.44 & 2 & No & B from expt \\
\hline
\end{tabular}


Table A4. Molecular orbital data for $p$-xylene. Double-ionization threshold $=23.11 \mathrm{eV}$ from CCSD(T)/cc-pV(T+d)Z (to singlet dication).

\begin{tabular}{|c|c|c|c|c|c|}
\hline MO & $\mathrm{B} / \mathrm{eV}$ & U/eV & $\mathbf{N}$ & DblIon & Remarks \\
\hline 1 & 305.74 & 435.97 & 2 & Yes & \\
\hline 2 & 305.74 & 435.97 & 2 & Yes & \\
\hline 3 & 305.51 & 435.8 & 2 & Yes & \\
\hline 4 & 305.51 & 435.86 & 2 & Yes & \\
\hline 5 & 305.48 & 436.16 & 2 & Yes & \\
\hline 6 & 305.48 & 436.21 & 2 & Yes & \\
\hline 7 & 305.4 & 436.02 & 2 & Yes & \\
\hline 8 & 305.4 & 436.01 & 2 & Yes & \\
\hline 9 & 31.33 & 38.97 & 2 & Yes & B from P3 \\
\hline 10 & 28.83 & 39.17 & 2 & Yes & \\
\hline 11 & 27.44 & 41.92 & 2 & Yes & \\
\hline 12 & 26.43 & 36.02 & 2 & Yes & B from P3 \\
\hline 13 & 24.15 & 39.48 & 2 & Yes & B from P3 \\
\hline 14 & 26.71 & 37.61 & 2 & Yes & B from P3 \\
\hline 15 & 18.62 & 37.42 & 2 & No & B from P3 \\
\hline 16 & 16.2 & 29.34 & 2 & No & B from P3 \\
\hline 17 & 15.55 & 34.25 & 2 & No & B from P3 \\
\hline 18 & 15.15 & 34.12 & 2 & No & B from P3 \\
\hline 19 & 14.04 & 32.48 & 2 & No & B from P3 \\
\hline 20 & 14.21 & 24.38 & 2 & No & B from P3 \\
\hline 21 & 14.17 & 25.2 & 2 & No & B from P3 \\
\hline 22 & 13.82 & 27.8 & 2 & No & B from P3 \\
\hline 23 & 13.36 & 36.63 & 2 & No & B from P3 \\
\hline 24 & 12.94 & 32.2 & 2 & No & B from P3 \\
\hline 25 & 11.75 & 40.59 & 2 & No & B from P3 \\
\hline 26 & 11.71 & 37.22 & 2 & No & B from P3 \\
\hline 27 & 11.58 & 25.68 & 2 & No & B from P3 \\
\hline 28 & 8.99 & 28.05 & 2 & No & B from P3 \\
\hline 29 & 8.44 & 29.94 & 2 & No & B from expt \\
\hline
\end{tabular}


Table A5. Molecular orbital data for styrene. Double-ionization threshold $=22.65 \mathrm{eV}$ from CCSD(T)/cc-pV(T+d)Z (to singlet dication).

\begin{tabular}{|c|c|c|c|c|c|}
\hline MO & $\mathrm{B} / \mathrm{eV}$ & $\mathrm{U} / \mathrm{eV}$ & $\mathbf{N}$ & DblIon & Remarks \\
\hline 1 & 306.03 & 435.95 & 2 & Yes & \\
\hline 2 & 305.88 & 436.0 & 2 & Yes & \\
\hline 3 & 305.79 & 435.72 & 2 & Yes & \\
\hline 4 & 305.78 & 435.81 & 2 & Yes & \\
\hline 5 & 305.76 & 436.02 & 2 & Yes & \\
\hline 6 & 305.74 & 436.15 & 2 & Yes & \\
\hline 7 & 305.73 & 436.3 & 2 & Yes & \\
\hline 8 & 305.53 & 436.03 & 2 & Yes & \\
\hline 9 & 31.63 & 39.26 & 2 & Yes & B from P3 \\
\hline 10 & 29.19 & 40.76 & 2 & Yes & \\
\hline 11 & 27.65 & 42.02 & 2 & Yes & B from P3 \\
\hline 12 & 26.99 & 42.77 & 2 & Yes & \\
\hline 13 & 23.25 & 38.53 & 2 & Yes & B from P3 \\
\hline 14 & 18.71 & 38.56 & 2 & No & B from P2 \\
\hline 15 & 18.36 & 35.56 & 2 & No & B from P3 \\
\hline 16 & 16.96 & 27.14 & 2 & No & B from P3 \\
\hline 17 & 15.84 & 32.73 & 2 & No & B from P3 \\
\hline 18 & 15.37 & 36.41 & 2 & No & B from P3 \\
\hline 19 & 14.8 & 32.45 & 2 & No & B from P3 \\
\hline 20 & 14.31 & 32.76 & 2 & No & B from P3 \\
\hline 21 & 14.09 & 37.31 & 2 & No & B from P3 \\
\hline 22 & 12.92 & 33.48 & 2 & No & B from P3 \\
\hline 23 & 12.48 & 23.56 & 2 & No & B from P3 \\
\hline 24 & 12.12 & 38.58 & 2 & No & B from P3 \\
\hline 25 & 11.96 & 36.84 & 2 & No & B from P3 \\
\hline 26 & 10.55 & 26.01 & 2 & No & B from expt \\
\hline 27 & 9.27 & 28.1 & 2 & No & B from expt \\
\hline 28 & 8.49 & 29.4 & 2 & No & B from expt \\
\hline
\end{tabular}


Table A6. Molecular orbital data for 1-hexene. Double-ionization threshold = $25.22 \mathrm{eV}$ from CCSD(T)/cc-pV(T+d)Z (to singlet dication).

\begin{tabular}{llllll}
\hline MO & B/eV & U/eV & N & DblIon & Remarks \\
\hline 1 & 305.6 & 435.98 & 2 & Yes & \\
2 & 305.46 & 436.04 & 2 & Yes & \\
3 & 305.34 & 435.96 & 2 & Yes & \\
4 & 305.33 & 436.16 & 2 & Yes & \\
5 & 305.24 & 436.02 & 2 & Yes & \\
6 & 305.15 & 436.03 & 2 & Yes & \\
7 & 29.69 & 35.88 & 2 & Yes & B from P3 \\
8 & 28.37 & 37.54 & 2 & Yes & \\
9 & 26.3 & 37.58 & 2 & Yes & \\
10 & 20.54 & 37.0 & 2 & No & B from P2 \\
11 & 21.59 & 32.57 & 2 & No & B from P3 \\
12 & 18.33 & 32.15 & 2 & No & B from P3 \\
13 & 16.12 & 24.25 & 2 & No & B from P3 \\
14 & 15.38 & 25.9 & 2 & No & B from P3 \\
15 & 14.71 & 30.17 & 2 & No & B from P3 \\
16 & 14.52 & 28.11 & 2 & No & B from P3 \\
17 & 13.84 & 29.7 & 2 & No & B from P3 \\
18 & 13.29 & 30.34 & 2 & No & B from P3 \\
19 & 12.65 & 29.56 & 2 & No & B from P3 \\
20 & 12.21 & 29.93 & 2 & No & B from P3 \\
21 & 11.8 & 33.07 & 2 & No & B from P3 \\
22 & 11.62 & 28.28 & 2 & No & B from P3 \\
23 & 11.36 & 35.64 & 2 & No & B from P3 \\
24 & 9.65 & 28.55 & 2 & No & B from expt \\
\hline & & & & &
\end{tabular}

Table A7. Molecular orbital data for 2-hexene. Double-ionization threshold $=25.23$ eV from P3/cc-pVTZ (to singlet dication).

\begin{tabular}{llllll}
\hline MO & B/eV & U/eV & N & DblIon & Remarks \\
\hline 1 & 305.41 & 435.89 & 2 & Yes & \\
2 & 305.37 & 435.99 & 2 & Yes & \\
3 & 305.31 & 436.09 & 2 & Yes & \\
4 & 305.31 & 436.12 & 2 & Yes & \\
5 & 305.3 & 436.06 & 2 & Yes & \\
6 & 305.13 & 436.03 & 2 & Yes & \\
7 & 29.63 & 37.08 & 2 & Yes & \\
8 & 28.27 & 36.51 & 2 & Yes & B from P3 \\
9 & 22.56 & 36.12 & 2 & No & B from P2 \\
10 & 24.33 & 38.14 & 2 & No & B from P3 \\
11 & 19.42 & 34.29 & 2 & No & B from P3 \\
12 & 18.04 & 30.3 & 2 & No & B from P3 \\
13 & 15.71 & 24.7 & 2 & No & B from P3 \\
14 & 15.22 & 28.9 & 2 & No & B from P3 \\
15 & 14.53 & 29.23 & 2 & No & B from P3 \\
16 & 14.16 & 26.5 & 2 & No & B from P3 \\
17 & 14.01 & 26.93 & 2 & No & B from P3 \\
18 & 13.31 & 31.47 & 2 & No & B from P3 \\
19 & 13.02 & 28.7 & 2 & No & B from P3 \\
20 & 12.37 & 31.8 & 2 & No & B from P3 \\
21 & 11.95 & 30.99 & 2 & No & B from P3 \\
22 & 11.66 & 28.82 & 2 & No & B from P3 \\
23 & 11.43 & 36.2 & 2 & No & B from P3 \\
24 & 9.16 & 29.63 & 2 & No & B from expt \\
\hline & & & & & \\
\hline
\end{tabular}


Table A8. Molecular orbital data for 3-hexene. Double-ionization threshold = $25.08 \mathrm{eV}$ from CCSD(T)/cc-pV(T+d)Z (to singlet dication).

\begin{tabular}{llllll}
\hline MO & B/eV & U/eV & N & DblIon & Remarks \\
\hline 1 & 305.44 & 436.01 & 2 & Yes & \\
2 & 305.44 & 436.03 & 2 & Yes & \\
3 & 305.36 & 435.81 & 2 & Yes & \\
4 & 305.32 & 436.26 & 2 & Yes & \\
5 & 305.17 & 436.04 & 2 & Yes & \\
6 & 305.17 & 436.04 & 2 & Yes & \\
7 & 29.66 & 37.51 & 2 & Yes & B from P3 \\
8 & 28.22 & 35.22 & 2 & Yes & \\
9 & 26.3 & 37.99 & 2 & Yes & \\
10 & 25.76 & 35.89 & 2 & Yes & B from P3 \\
11 & 19.41 & 36.31 & 2 & No & B from P2 \\
12 & 18.05 & 29.59 & 2 & No & B from P3 \\
13 & 15.59 & 26.84 & 2 & No & B from P3 \\
14 & 15.43 & 26.21 & 2 & No & B from P3 \\
15 & 14.63 & 27.09 & 2 & No & B from P3 \\
16 & 14.37 & 26.84 & 2 & No & B from P3 \\
17 & 13.42 & 31.68 & 2 & No & B from P3 \\
18 & 13.22 & 29.56 & 2 & No & B from P3 \\
19 & 12.63 & 32.37 & 2 & No & B from P3 \\
20 & 12.68 & 29.14 & 2 & No & B from P3 \\
21 & 12.04 & 29.39 & 2 & No & B from P3 \\
22 & 11.96 & 29.69 & 2 & No & B from P3 \\
23 & 11.33 & 35.03 & 2 & No & B from P3 \\
24 & 9.14 & 29.95 & 2 & No & B from expt \\
\hline & & & & &
\end{tabular}

Table A9. Molecular orbital data for 2-methyl-1-pentene. Double-ionization threshold = 26.35 eV P3/cc-pVTZ (to triplet dication).

\begin{tabular}{llllll}
\hline MO & B/eV & U/eV & N & DblIon & Remarks \\
\hline 1 & 305.75 & 436.03 & 2 & Yes & \\
2 & 305.41 & 436.01 & 2 & Yes & \\
3 & 305.38 & 436.09 & 2 & Yes & \\
4 & 305.32 & 436.01 & 2 & Yes & \\
5 & 305.18 & 436.03 & 2 & Yes & \\
6 & 305.0 & 436.0 & 2 & Yes & \\
7 & 29.93 & 36.81 & 2 & Yes & \\
8 & 28.11 & 36.55 & 2 & Yes & \\
9 & 25.41 & 35.78 & 2 & No & B from P3 \\
10 & 25.02 & 37.38 & 2 & No & B from P3 \\
11 & 19.64 & 34.03 & 2 & No & B from P3 \\
12 & 17.44 & 33.95 & 2 & No & B from P3 \\
13 & 15.84 & 24.09 & 2 & No & B from P3 \\
14 & 15.38 & 27.1 & 2 & No & B from P3 \\
15 & 14.58 & 29.35 & 2 & No & B from P3 \\
16 & 14.47 & 25.56 & 2 & No & B from P3 \\
17 & 14.06 & 26.92 & 2 & No & B from P3 \\
18 & 13.21 & 30.09 & 2 & No & B from P3 \\
19 & 12.95 & 28.88 & 2 & No & B from P3 \\
20 & 12.59 & 33.68 & 2 & No & B from P3 \\
21 & 11.99 & 31.38 & 2 & No & B from P3 \\
22 & 11.68 & 29.48 & 2 & No & B from P3 \\
23 & 11.36 & 36.33 & 2 & No & B from P3 \\
24 & 9.31 & 28.95 & 2 & No & B from CCSD(T) \\
\hline & & & & & \\
\hline
\end{tabular}


Table A10. Molecular orbital data for 3-methyl-1-pentene. Double-ionization threshold $=25.68 \mathrm{eV}$ from CCSD(T)/cc-pV(T+d)Z (to singlet dication).

\begin{tabular}{llllll}
\hline MO & $\mathbf{B / e V}$ & $\mathbf{U} / \mathbf{e V}$ & $\mathbf{N}$ & DblIon & Remarks \\
\hline 1 & 305.63 & 436.04 & 2 & Yes & \\
2 & 305.55 & 436.01 & 2 & Yes & \\
3 & 305.35 & 436.06 & 2 & Yes & \\
4 & 305.24 & 436.03 & 2 & Yes & \\
5 & 305.16 & 436.03 & 2 & Yes & \\
6 & 305.16 & 436.02 & 2 & Yes & \\
7 & 29.97 & 35.84 & 2 & Yes & B from P3 \\
8 & 27.78 & 38.22 & 2 & Yes & B from P3 \\
9 & 26.41 & 36.77 & 2 & Yes & \\
10 & 21.12 & 35.1 & 2 & No & B from P3 \\
11 & 19.24 & 35.82 & 2 & No & B from P3 \\
12 & 18.25 & 30.0 & 2 & No & B from P3 \\
13 & 15.75 & 26.77 & 2 & No & B from P3 \\
14 & 15.32 & 27.26 & 2 & No & B from P3 \\
15 & 14.92 & 28.04 & 2 & No & B from P3 \\
16 & 14.39 & 27.03 & 2 & No & B from P3 \\
17 & 13.51 & 29.69 & 2 & No & B from P3 \\
18 & 13.43 & 28.15 & 2 & No & B from P3 \\
19 & 13.01 & 29.93 & 2 & No & B from P3 \\
20 & 12.54 & 30.63 & 2 & No & B from P3 \\
21 & 11.61 & 31.31 & 2 & No & B from P3 \\
22 & 11.5 & 30.73 & 2 & No & B from P3 \\
23 & 11.27 & 35.87 & 2 & No & B from P3 \\
24 & 9.68 & 29.01 & 2 & No & B from CCSD(T) \\
\hline
\end{tabular}

Table A11. Molecular orbital data for 4-methyl-1-pentene. Double-ionization threshold $=25.46 \mathrm{eV}$ from CCSD(T)/cc-pV(T+d)Z (to triplet dication).

\begin{tabular}{llllll}
\hline MO & $\mathbf{B} / \mathbf{e V}$ & $\mathbf{U} / \mathbf{e V}$ & $\mathbf{N}$ & DblIon & Remarks \\
\hline 1 & 305.59 & 435.98 & 2 & Yes & \\
2 & 305.58 & 436.08 & 2 & Yes & \\
3 & 305.42 & 436.06 & 2 & Yes & \\
4 & 305.26 & 436.03 & 2 & Yes & \\
5 & 305.13 & 436.02 & 2 & Yes & \\
6 & 305.13 & 436.02 & 2 & Yes & \\
7 & 29.89 & 35.43 & 2 & Yes & B from P3 \\
8 & 28.2 & 38.86 & 2 & Yes & B from P3 \\
9 & 22.09 & 34.64 & 2 & No & B from P2 \\
10 & 21.54 & 37.47 & 2 & No & B from P2 \\
11 & 18.98 & 34.59 & 2 & No & B from P3 \\
12 & 18.15 & 31.57 & 2 & No & B from P3 \\
13 & 16.08 & 25.01 & 2 & No & B from P3 \\
14 & 15.12 & 27.34 & 2 & No & B from P3 \\
15 & 14.79 & 29.99 & 2 & No & B from P3 \\
16 & 14.65 & 26.49 & 2 & No & B from P3 \\
17 & 13.5 & 29.3 & 2 & No & B from P3 \\
18 & 13.31 & 28.37 & 2 & No & B from P3 \\
19 & 13.16 & 29.13 & 2 & No & B from P3 \\
20 & 12.45 & 30.06 & 2 & No & B from P3 \\
21 & 11.66 & 33.18 & 2 & No & B from P3 \\
22 & 11.5 & 31.05 & 2 & No & B from P3 \\
23 & 11.31 & 35.12 & 2 & No & B from P3 \\
24 & 9.69 & 28.56 & 2 & No & B from CCSD(T) \\
\hline
\end{tabular}


Table A12. Molecular orbital data for 2-methyl-2-pentene. Double-ionization threshold $=24.54 \mathrm{eV}$ from CCSD(T)/cc-pV(T+d)Z (to singlet dication).

\begin{tabular}{llllll}
\hline MO & $\mathbf{B / e V}$ & $\mathbf{U} / \mathbf{e V}$ & $\mathbf{N}$ & DblIon & Remarks \\
\hline 1 & 305.6 & 436.02 & 2 & Yes & \\
2 & 305.37 & 436.03 & 2 & Yes & \\
3 & 305.27 & 436.01 & 2 & Yes & \\
4 & 305.23 & 436.02 & 2 & Yes & \\
5 & 305.15 & 436.04 & 2 & Yes & \\
6 & 305.13 & 436.04 & 2 & Yes & \\
7 & 29.85 & 37.63 & 2 & Yes & B from P3 \\
8 & 27.96 & 35.53 & 2 & Yes & B from P3 \\
9 & 25.54 & 34.98 & 2 & Yes & B from P3 \\
10 & 21.53 & 37.44 & 2 & No & B from P2 \\
11 & 20.8 & 36.22 & 2 & No & B from P3 \\
12 & 17.11 & 32.62 & 2 & No & B from P3 \\
13 & 15.69 & 26.69 & 2 & No & B from P3 \\
14 & 15.03 & 27.29 & 2 & No & B from P3 \\
15 & 14.66 & 24.63 & 2 & No & B from P3 \\
16 & 14.31 & 26.9 & 2 & No & B from P3 \\
17 & 13.67 & 28.45 & 2 & No & B from P3 \\
18 & 13.34 & 26.95 & 2 & No & B from P3 \\
19 & 12.86 & 30.62 & 2 & No & B from P3 \\
20 & 12.64 & 31.75 & 2 & No & B from P3 \\
21 & 12.46 & 32.2 & 2 & No & B from P3 \\
22 & 11.92 & 31.24 & 2 & No & B from P3 \\
23 & 11.23 & 35.32 & 2 & No & B from P3 \\
24 & 8.88 & 29.93 & 2 & No & B from CCSD(T) \\
\hline
\end{tabular}

Table A13. Molecular orbital data for 3-methyl-2-pentene. Double-ionization threshold $=24.60 \mathrm{eV}$ from CCSD(T)/cc-pV(T+d)Z (to singlet dication).

\begin{tabular}{llllll}
\hline MO & B/eV & U/eV & N & DblIon & Remarks \\
\hline 1 & 305.52 & 435.99 & 2 & Yes & \\
2 & 305.37 & 436.06 & 2 & Yes & \\
3 & 305.25 & 436.01 & 2 & Yes & \\
4 & 305.24 & 435.95 & 2 & Yes & \\
5 & 305.21 & 436.12 & 2 & Yes & \\
6 & 305.14 & 436.03 & 2 & Yes & \\
7 & 29.9 & 37.36 & 2 & Yes & \\
8 & 27.61 & 35.82 & 2 & Yes & B from P3 \\
9 & 26.26 & 36.03 & 2 & Yes & B from P3 \\
10 & 24.51 & 35.9 & 2 & No & B from P3 \\
11 & 21.45 & 36.51 & 2 & No & B from P3 \\
12 & 17.03 & 33.1 & 2 & No & B from P3 \\
13 & 15.77 & 25.93 & 2 & No & B from P3 \\
14 & 15.19 & 25.84 & 2 & No & B from P3 \\
15 & 14.38 & 26.17 & 2 & No & B from P3 \\
16 & 13.82 & 30.28 & 2 & No & B from P3 \\
17 & 13.88 & 26.65 & 2 & No & B from P3 \\
18 & 13.63 & 27.39 & 2 & No & B from P3 \\
19 & 13.16 & 28.97 & 2 & No & B from P3 \\
20 & 12.56 & 31.78 & 2 & No & B from P3 \\
21 & 12.37 & 31.61 & 2 & No & B from P3 \\
22 & 11.86 & 30.99 & 2 & No & B from P3 \\
23 & 11.18 & 36.05 & 2 & No & B from P3 \\
24 & 8.88 & 29.98 & 2 & No & B from CCSD(T) \\
\hline
\end{tabular}


Table A14. Molecular orbital data for 4-methyl-2-pentene. Double-ionization threshold $=25.05 \mathrm{eV}$ from CCSD(T)/cc-pV(T+d)Z (to singlet dication).

\begin{tabular}{llllll}
\hline MO & $\mathbf{B} / \mathbf{e V}$ & $\mathbf{U} / \mathbf{e V}$ & $\mathbf{N}$ & DblIon & Remarks \\
\hline 1 & 305.59 & 436.07 & 2 & Yes & \\
2 & 305.42 & 435.92 & 2 & Yes & \\
3 & 305.31 & 436.03 & 2 & Yes & \\
4 & 305.28 & 436.09 & 2 & Yes & \\
5 & 305.11 & 436.02 & 2 & Yes & \\
6 & 305.11 & 436.03 & 2 & Yes & \\
7 & 29.81 & 36.78 & 2 & Yes & B from P3 \\
8 & 28.11 & 36.98 & 2 & Yes & B from P3 \\
9 & 22.21 & 34.78 & 2 & No & B from P3 \\
10 & 21.7 & 37.49 & 2 & No & B from P2 \\
11 & 18.86 & 37.07 & 2 & No & B from P2 \\
12 & 18.06 & 29.09 & 2 & No & B from P3 \\
13 & 15.65 & 26.92 & 2 & No & B from P3 \\
14 & 15.04 & 28.4 & 2 & No & B from P3 \\
15 & 14.2 & 25.79 & 2 & No & B from P3 \\
16 & 13.95 & 30.8 & 2 & No & B from P3 \\
17 & 13.83 & 27.05 & 2 & No & B from P3 \\
18 & 13.86 & 28.38 & 2 & No & B from P3 \\
19 & 13.36 & 26.83 & 2 & No & B from P3 \\
20 & 12.5 & 30.75 & 2 & No & B from P3 \\
21 & 11.92 & 32.64 & 2 & No & B from P3 \\
22 & 11.62 & 32.0 & 2 & No & B from P3 \\
23 & 11.3 & 34.56 & 2 & No & B from P3 \\
24 & 9.21 & 30.0 & 2 & No & B from CCSD(T) \\
\hline
\end{tabular}

Table A15. Molecular orbital data for 2,3-dimethyl-1-butene. Double-ionization threshold $=25.59 \mathrm{eV}$ from CCSD(T)/cc-pV(T+d)Z (to singlet dication).

\begin{tabular}{llllll}
\hline MO & B/eV & U/eV & N & DblIon & Remarks \\
\hline 1 & 305.71 & 436.01 & 2 & Yes & \\
2 & 305.65 & 436.11 & 2 & Yes & \\
3 & 305.31 & 436.01 & 2 & Yes & \\
4 & 305.17 & 436.02 & 2 & Yes & \\
5 & 305.17 & 436.03 & 2 & Yes & \\
6 & 305.0 & 436.0 & 2 & Yes & \\
7 & 30.12 & 36.46 & 2 & Yes & B from P3 \\
8 & 27.73 & 37.43 & 2 & Yes & B from P3 \\
9 & 25.33 & 36.46 & 2 & No & \\
10 & 22.85 & 34.73 & 2 & No & B from P3 \\
11 & 19.69 & 35.48 & 2 & No & B from P3 \\
12 & 17.29 & 33.35 & 2 & No & B from P3 \\
13 & 15.77 & 26.64 & 2 & No & B from P3 \\
14 & 15.22 & 27.18 & 2 & No & B from P3 \\
15 & 14.52 & 25.14 & 2 & No & B from P3 \\
16 & 14.27 & 27.73 & 2 & No & B from P3 \\
17 & 13.86 & 27.1 & 2 & No & B from P3 \\
18 & 13.65 & 29.0 & 2 & No & B from P3 \\
19 & 13.17 & 27.86 & 2 & No & B from P3 \\
20 & 12.73 & 31.6 & 2 & No & B from P3 \\
21 & 11.94 & 32.92 & 2 & No & B from P3 \\
22 & 11.59 & 33.25 & 2 & No & B from P3 \\
23 & 11.23 & 34.76 & 2 & No & B from P3 \\
24 & 9.31 & 29.2 & 2 & No & B from CCSD(T) \\
\hline
\end{tabular}


Table A16. Molecular orbital data for 3,3-dimethyl-1-butene. Double-ionization threshold = $25.96 \mathrm{eV}$ from CCSD(T)/cc-pV(T+d)Z (to singlet dication).

\begin{tabular}{|c|c|c|c|c|c|}
\hline MO & B/eV & U/eV & $\mathbf{N}$ & DblIon & Remarks \\
\hline 1 & 305.9 & 436.11 & 2 & Yes & \\
\hline 2 & 305.54 & 435.99 & 2 & Yes & \\
\hline 3 & 305.21 & 436.02 & 2 & Yes & \\
\hline 4 & 305.17 & 436.02 & 2 & Yes & \\
\hline 5 & 305.17 & 436.03 & 2 & Yes & \\
\hline 6 & 305.11 & 436.01 & 2 & Yes & \\
\hline 7 & 30.71 & 35.62 & 2 & Yes & B from P3 \\
\hline 8 & 27.3 & 39.93 & 2 & Yes & B from P3 \\
\hline 9 & 25.36 & 34.39 & 2 & No & \\
\hline 10 & 22.27 & 34.58 & 2 & No & B from P3 \\
\hline 11 & 22.26 & 35.56 & 2 & No & \\
\hline 12 & 17.39 & 33.46 & 2 & No & B from P3 \\
\hline 13 & 15.79 & 25.63 & 2 & No & B from P3 \\
\hline 14 & 15.36 & 29.41 & 2 & No & B from P3 \\
\hline 15 & 14.9 & 25.8 & 2 & No & B from P3 \\
\hline 16 & 14.43 & 26.86 & 2 & No & B from P3 \\
\hline 17 & 13.56 & 26.61 & 2 & No & B from P3 \\
\hline 18 & 13.23 & 29.38 & 2 & No & B from P3 \\
\hline 19 & 13.17 & 29.21 & 2 & No & B from P3 \\
\hline 20 & 12.83 & 28.37 & 2 & No & B from P3 \\
\hline 21 & 11.71 & 33.04 & 2 & No & B from P3 \\
\hline 22 & 11.44 & 35.48 & 2 & No & B from P3 \\
\hline 23 & 11.3 & 34.22 & 2 & No & B from P3 \\
\hline 24 & 9.69 & 28.64 & 2 & No & B from CCSD(T) \\
\hline
\end{tabular}

Table A17. Molecular orbital data for 2,3-dimethyl-2-butene. Double-ionization threshold $=24.00 \mathrm{eV}$ from CCSD(T)/cc-pV(T+d)Z (to singlet dication).

\begin{tabular}{llllll}
\hline MO & $\mathbf{B} / \mathbf{e V}$ & $\mathbf{U} / \mathbf{e V}$ & $\mathbf{N}$ & DblIon & Remarks \\
\hline 1 & 305.5 & 435.83 & 2 & Yes & \\
2 & 305.46 & 436.26 & 2 & Yes & \\
3 & 305.17 & 436.0 & 2 & Yes & \\
4 & 305.17 & 436.01 & 2 & Yes & \\
5 & 305.17 & 436.03 & 2 & Yes & \\
6 & 305.17 & 436.03 & 2 & Yes & \\
7 & 30.0 & 37.55 & 2 & Yes & \\
8 & 27.17 & 35.61 & 2 & Yes & B from P3 \\
9 & 25.72 & 33.83 & 2 & Yes & \\
10 & 24.88 & 36.35 & 2 & Yes & \\
11 & 23.23 & 38.21 & 2 & No & \\
12 & 16.35 & 35.9 & 2 & No & B from P3 \\
13 & 15.77 & 26.2 & 2 & No & B from P3 \\
14 & 14.87 & 23.11 & 2 & No & B from P3 \\
15 & 14.69 & 27.75 & 2 & No & B from P3 \\
16 & 14.24 & 24.44 & 2 & No & B from P3 \\
17 & 13.35 & 28.45 & 2 & No & B from P3 \\
18 & 13.24 & 26.83 & 2 & No & B from P3 \\
19 & 13.12 & 27.29 & 2 & No & B from P3 \\
20 & 12.84 & 30.96 & 2 & No & B from P3 \\
21 & 12.65 & 30.5 & 2 & No & B from P3 \\
22 & 12.71 & 35.74 & 2 & No & B from expt \\
23 & 10.96 & 37.74 & 2 & No & B from expt \\
24 & 8.44 & 29.93 & 2 & No & B from expt \\
\hline
\end{tabular}


Table A18. Molecular orbital data for 2-ethyl-1-butene. Double-ionization threshold = $25.74 \mathrm{eV}$ from CCSD(T)/cc-pV(T+d)Z (to singlet dication).

\begin{tabular}{llllll}
\hline MO & $\mathbf{B / e V}$ & $\mathbf{U} / \mathbf{e V}$ & $\mathbf{N}$ & DblIon & Remarks \\
\hline 1 & 305.68 & 436.02 & 2 & Yes & \\
2 & 305.46 & 436.03 & 2 & Yes & \\
3 & 305.46 & 436.06 & 2 & Yes & \\
4 & 305.22 & 436.03 & 2 & Yes & \\
5 & 305.22 & 436.03 & 2 & Yes & \\
6 & 305.01 & 436.0 & 2 & Yes & \\
7 & 30.03 & 36.84 & 2 & Yes & B from P3 \\
8 & 27.61 & 35.47 & 2 & Yes & \\
9 & 26.6 & 38.0 & 2 & Yes & \\
10 & 20.48 & 36.59 & 2 & No & B from P3 \\
11 & 20.55 & 33.76 & 2 & No & B from P3 \\
12 & 17.3 & 33.7 & 2 & No & B from P3 \\
13 & 15.77 & 25.67 & 2 & No & B from P3 \\
14 & 15.62 & 25.01 & 2 & No & B from P3 \\
15 & 14.42 & 28.69 & 2 & No & B from P3 \\
16 & 14.22 & 27.41 & 2 & No & B from P3 \\
17 & 14.26 & 26.87 & 2 & No & B from P3 \\
18 & 12.98 & 29.95 & 2 & No & B from P3 \\
19 & 12.97 & 29.3 & 2 & No & B from P3 \\
20 & 12.47 & 34.22 & 2 & No & B from P3 \\
21 & 12.24 & 29.07 & 2 & No & B from P3 \\
22 & 11.77 & 31.86 & 2 & No & B from P3 \\
23 & 11.24 & 34.59 & 2 & No & B from P3 \\
24 & 9.3 & 29.27 & 2 & No & B from CCSD(T) \\
\hline
\end{tabular}

Table A19. Molecular orbital data for 1-heptene. Double-ionization threshold = $24.47 \mathrm{eV}$ from P3 (to triplet or singlet dication).

\begin{tabular}{llllll}
\hline MO & $\mathbf{B} / \mathbf{e V}$ & $\mathbf{U} / \mathbf{e V}$ & $\mathbf{N}$ & DblIon & Remarks \\
\hline 1 & 305.6 & 435.98 & 2 & Yes & \\
2 & 305.45 & 436.04 & 2 & Yes & \\
3 & 305.33 & 436.04 & 2 & Yes & \\
4 & 305.3 & 436.0 & 2 & Yes & \\
5 & 305.27 & 436.13 & 2 & Yes & \\
6 & 305.23 & 436.02 & 2 & Yes & \\
7 & 305.13 & 436.03 & 2 & Yes & \\
8 & 29.74 & 35.48 & 2 & Yes & B from P3 \\
9 & 28.74 & 36.97 & 2 & Yes & \\
10 & 27.11 & 37.52 & 2 & Yes & B from P3 \\
11 & 24.38 & 37.37 & 2 & No & B from P3 \\
12 & 19.98 & 36.69 & 2 & No & B from P3 \\
13 & 19.19 & 31.09 & 2 & No & B from P3 \\
14 & 18.3 & 32.24 & 2 & No & B from P3 \\
15 & 16.18 & 23.53 & 2 & No & B from P3 \\
16 & 15.54 & 25.7 & 2 & No & B from P3 \\
17 & 14.88 & 29.17 & 2 & No & B from P3 \\
18 & 14.57 & 27.05 & 2 & No & B from P3 \\
19 & 14.1 & 29.36 & 2 & No & B from P3 \\
20 & 13.76 & 31.42 & 2 & No & B from P3 \\
21 & 13.06 & 29.64 & 2 & No & B from P3 \\
22 & 12.67 & 30.38 & 2 & No & B from P3 \\
23 & 12.25 & 31.57 & 2 & No & B from P3 \\
24 & 11.9 & 29.31 & 2 & No & B from P3 \\
25 & 11.56 & 31.61 & 2 & No & B from P3 \\
26 & 11.52 & 27.94 & 2 & No & B from P3 \\
27 & 11.13 & 38.04 & 2 & No & B from P3 \\
28 & 9.68 & 28.63 & 2 & No & B from Pxpt \\
\hline & & & & &
\end{tabular}


Table A20. Molecular orbital data for 2-heptene. Double-ionization threshold = $24.37 \mathrm{eV}$ from CCSD(T)/cc-pVTZ (to singlet dication).

\begin{tabular}{llllll}
\hline MO & B/eV & U/eV & N & DblIon & Remarks \\
\hline 1 & 305.41 & 435.88 & 2 & Yes & \\
2 & 305.36 & 436.0 & 2 & Yes & \\
3 & 305.31 & 436.13 & 2 & Yes & \\
4 & 305.3 & 436.06 & 2 & Yes & \\
5 & 305.28 & 436.0 & 2 & Yes & \\
6 & 305.25 & 436.12 & 2 & Yes & \\
7 & 305.11 & 436.03 & 2 & Yes & \\
8 & 29.7 & 36.49 & 2 & Yes & B from P3 \\
9 & 28.67 & 36.7 & 2 & Yes & \\
10 & 26.99 & 35.83 & 2 & Yes & \\
11 & 21.64 & 37.4 & 2 & No & B from P2 \\
12 & 20.04 & 37.85 & 2 & No & B from P2 \\
13 & 19.18 & 32.18 & 2 & No & B from P3 \\
14 & 18.0 & 30.55 & 2 & No & B from P3 \\
15 & 15.9 & 23.57 & 2 & No & B from P3 \\
16 & 15.04 & 29.72 & 2 & No & B from P3 \\
17 & 14.8 & 28.8 & 2 & No & B from P3 \\
18 & 14.53 & 26.79 & 2 & No & B from P3 \\
19 & 14.1 & 25.38 & 2 & No & B from P3 \\
20 & 13.68 & 28.44 & 2 & No & B from P3 \\
21 & 13.26 & 32.65 & 2 & No & B from P3 \\
22 & 12.84 & 30.21 & 2 & No & B from P3 \\
23 & 12.39 & 30.12 & 2 & No & B from P3 \\
24 & 12.02 & 30.37 & 2 & No & B from P3 \\
25 & 11.69 & 32.94 & 2 & No & B from P3 \\
26 & 11.52 & 28.24 & 2 & No & B from P3 \\
27 & 11.18 & 36.8 & 2 & No & B from P3 \\
28 & 9.11 & 29.75 & 2 & No & B from expt \\
\hline & & & & &
\end{tabular}

Table A21. Molecular orbital data for 3-heptene. Double-ionization threshold = $24.98 \mathrm{eV}$ from P3 (to singlet dication).

\begin{tabular}{llllll}
\hline MO & $\mathbf{B} / \mathbf{e V}$ & $\mathbf{U} / \mathbf{e V}$ & $\mathbf{N}$ & DbIIon & Remarks \\
\hline 1 & 305.43 & 436.02 & 2 & Yes & \\
2 & 305.37 & 435.91 & 2 & Yes & \\
3 & 305.36 & 435.89 & 2 & Yes & \\
4 & 305.32 & 436.1 & 2 & Yes & \\
5 & 305.31 & 436.26 & 2 & Yes & \\
6 & 305.16 & 436.04 & 2 & Yes & \\
7 & 305.13 & 436.03 & 2 & Yes & \\
8 & 29.73 & 37.05 & 2 & No & \\
9 & 28.62 & 35.29 & 2 & No & B from P3 \\
10 & 27.04 & 37.13 & 2 & No & \\
11 & 24.95 & 37.23 & 2 & No & B from P3 \\
12 & 20.25 & 36.43 & 2 & No & B from P3 \\
13 & 19.3 & 34.27 & 2 & No & B from P3 \\
14 & 18.02 & 29.72 & 2 & No & B from P3 \\
15 & 15.77 & 24.85 & 2 & No & B from P3 \\
16 & 15.41 & 27.09 & 2 & No & B from P3 \\
17 & 14.84 & 27.31 & 2 & No & B from P3 \\
18 & 14.56 & 27.53 & 2 & No & B from P3 \\
19 & 13.89 & 28.44 & 2 & No & B from P3 \\
20 & 13.54 & 27.87 & 2 & No & B from P3 \\
21 & 13.04 & 32.87 & 2 & No & B from P3 \\
22 & 12.65 & 30.21 & 2 & No & B from P3 \\
23 & 12.49 & 31.32 & 2 & No & B from P3 \\
24 & 11.97 & 30.09 & 2 & No & B from P3 \\
25 & 11.93 & 30.63 & 2 & No & B from P3 \\
26 & 11.66 & 28.65 & 2 & No & B from P3 \\
27 & 11.2 & 36.62 & 2 & No & B from P3 \\
28 & 9.05 & 30.15 & 2 & No & B from expt \\
\hline & & & & &
\end{tabular}


Table A22. Molecular orbital data for 2-methyl-1-hexene. Double-ionization threshold $=24.64 \mathrm{eV}$ from P3 (to singlet or triplet dication).

\begin{tabular}{|c|c|c|c|c|c|}
\hline MO & $\mathrm{B} / \mathrm{eV}$ & U/eV & $\mathbf{N}$ & DblIon & Remarks \\
\hline 1 & 305.74 & 436.03 & 2 & Yes & \\
\hline 2 & 305.41 & 436.03 & 2 & Yes & \\
\hline 3 & 305.33 & 435.98 & 2 & Yes & \\
\hline 4 & 305.32 & 436.01 & 2 & Yes & \\
\hline 5 & 305.31 & 436.14 & 2 & Yes & \\
\hline 6 & 305.15 & 436.03 & 2 & Yes & \\
\hline 7 & 305.0 & 436.0 & 2 & Yes & \\
\hline 8 & 29.98 & 36.45 & 2 & Yes & \\
\hline 9 & 28.67 & 36.29 & 2 & Yes & \\
\hline 10 & 26.42 & 36.37 & 2 & Yes & \\
\hline 11 & 25.31 & 36.73 & 2 & Yes & \\
\hline 12 & 23.59 & 36.67 & 2 & No & \\
\hline 13 & 19.29 & 32.36 & 2 & No & B from P3 \\
\hline 14 & 17.38 & 34.49 & 2 & No & B from P3 \\
\hline 15 & 16.01 & 23.21 & 2 & No & B from P3 \\
\hline 16 & 15.25 & 27.22 & 2 & No & B from P3 \\
\hline 17 & 14.94 & 27.85 & 2 & No & B from P3 \\
\hline 18 & 14.73 & 26.08 & 2 & No & B from P3 \\
\hline 19 & 14.05 & 26.31 & 2 & No & B from P3 \\
\hline 20 & 13.68 & 29.21 & 2 & No & B from P3 \\
\hline 21 & 13.33 & 31.0 & 2 & No & B from P3 \\
\hline 22 & 12.86 & 30.64 & 2 & No & B from P3 \\
\hline 23 & 12.39 & 30.75 & 2 & No & B from P3 \\
\hline 24 & 12.09 & 31.77 & 2 & No & B from P3 \\
\hline 25 & 11.75 & 32.91 & 2 & No & B from P3 \\
\hline 26 & 11.54 & 28.56 & 2 & No & B from P3 \\
\hline 27 & 11.14 & 36.87 & 2 & No & B from P3 \\
\hline 28 & 9.28 & 29.05 & 2 & No & B from $\operatorname{CCSD}(\mathrm{T})$ \\
\hline
\end{tabular}

Table A23. Molecular orbital data for 3-methyl-1-hexene. Double-ionization threshold $=25.10 \mathrm{eV}$ from CCSD(T)/cc-pV(T+d)Z (to singlet dication).

\begin{tabular}{llllll}
\hline MO & B/eV & U/eV & N & DblIon & Remarks \\
\hline 1 & 305.63 & 436.05 & 2 & Yes & \\
2 & 305.55 & 436.01 & 2 & Yes & \\
3 & 305.31 & 436.0 & 2 & Yes & \\
4 & 305.28 & 436.11 & 2 & Yes & \\
5 & 305.23 & 436.03 & 2 & Yes & \\
6 & 305.16 & 436.02 & 2 & Yes & \\
7 & 305.14 & 436.03 & 2 & Yes & \\
8 & 30.03 & 35.55 & 2 & Yes & B from P3 \\
9 & 28.38 & 36.73 & 2 & Yes & \\
10 & 26.91 & 38.33 & 2 & Yes & B from P3 \\
11 & 21.76 & 35.54 & 2 & No & B from P2 \\
12 & 19.95 & 35.86 & 2 & No & B from P2 \\
13 & 19.01 & 34.01 & 2 & No & B from P3 \\
14 & 18.22 & 30.34 & 2 & No & B from P3 \\
15 & 15.88 & 25.75 & 2 & No & B from P3 \\
16 & 15.31 & 26.05 & 2 & No & B from P3 \\
17 & 15.07 & 28.97 & 2 & No & B from P3 \\
18 & 14.58 & 27.85 & 2 & No & B from P3 \\
19 & 14.09 & 27.26 & 2 & No & B from P3 \\
20 & 13.51 & 29.22 & 2 & No & B from P3 \\
21 & 13.31 & 28.42 & 2 & No & B from P3 \\
22 & 12.84 & 31.82 & 2 & No & B from P3 \\
23 & 12.38 & 30.59 & 2 & No & B from P3 \\
24 & 11.96 & 30.51 & 2 & No & B from P3 \\
25 & 11.48 & 31.53 & 2 & No & B from P3 \\
26 & 11.42 & 30.81 & 2 & No & B from P3 \\
27 & 11.05 & 36.31 & 2 & No & B from P3 \\
28 & 9.64 & 29.16 & 2 & No & B from CCSD(T) \\
\hline & & & & &
\end{tabular}


Table A24. Molecular orbital data for 4-methyl-1-hexene. Double-ionization threshold = $24.87 \mathrm{eV}$ from P3 (to triplet dication).

\begin{tabular}{llllll}
\hline MO & B/eV & U/eV & N & DblIon & Remarks \\
\hline 1 & 305.61 & 435.98 & 2 & Yes & \\
2 & 305.53 & 436.08 & 2 & Yes & \\
3 & 305.41 & 436.06 & 2 & Yes & \\
4 & 305.29 & 436.05 & 2 & Yes & \\
5 & 305.25 & 436.03 & 2 & Yes & \\
6 & 305.13 & 436.03 & 2 & Yes & \\
7 & 304.98 & 436.02 & 2 & Yes & \\
8 & 30.0 & 35.04 & 2 & Yes & \\
9 & 28.46 & 38.26 & 2 & Yes & \\
10 & 26.71 & 36.75 & 2 & Yes & B from P3 \\
11 & 25.89 & 36.05 & 2 & Yes & B from P3 \\
12 & 20.6 & 35.79 & 2 & No & B from P3 \\
13 & 18.56 & 34.09 & 2 & No & B from P3 \\
14 & 18.3 & 30.74 & 2 & No & B from P3 \\
15 & 15.77 & 26.42 & 2 & No & B from P3 \\
16 & 15.4 & 26.08 & 2 & No & B from P3 \\
17 & 15.16 & 25.2 & 2 & No & B from P3 \\
18 & 14.67 & 29.57 & 2 & No & B from P3 \\
19 & 13.95 & 29.37 & 2 & No & B from P3 \\
20 & 13.54 & 29.51 & 2 & No & B from P3 \\
21 & 13.29 & 28.23 & 2 & No & B from P3 \\
22 & 12.74 & 30.07 & 2 & No & B from P3 \\
23 & 12.34 & 29.92 & 2 & No & B from P3 \\
24 & 12.09 & 30.38 & 2 & No & B from P3 \\
25 & 11.41 & 33.92 & 2 & No & B from P3 \\
26 & 11.35 & 30.48 & 2 & No & B from P3 \\
27 & 11.03 & 36.12 & 2 & No & B from P3 \\
28 & 9.66 & 28.62 & 2 & No & B from CCSD(T) \\
\hline & & & & &
\end{tabular}

Table A25. Molecular orbital data for 5-methyl-1-hexene. Double-ionization threshold $=24.71 \mathrm{eV}$ from P3 (to singlet dication).

\begin{tabular}{|c|c|c|c|c|c|}
\hline MO & $\mathrm{B} / \mathrm{eV}$ & $\mathrm{U} / \mathrm{eV}$ & $\mathbf{N}$ & DblIon & Remarks \\
\hline 1 & 305.59 & 435.98 & 2 & Yes & \\
\hline 2 & 305.53 & 436.08 & 2 & Yes & \\
\hline 3 & 305.42 & 436.03 & 2 & Yes & \\
\hline 4 & 305.31 & 436.08 & 2 & Yes & \\
\hline 5 & 305.22 & 436.02 & 2 & Yes & \\
\hline 6 & 305.11 & 436.02 & 2 & Yes & \\
\hline 7 & 305.1 & 436.02 & 2 & Yes & \\
\hline 8 & 29.9 & 34.91 & 2 & Yes & B from P3 \\
\hline 9 & 28.73 & 37.98 & 2 & Yes & \\
\hline 10 & 26.39 & 38.2 & 2 & Yes & \\
\hline 11 & 22.58 & 34.61 & 2 & No & B from OVGF \\
\hline 12 & 23.67 & 35.69 & 2 & No & B from P3 \\
\hline 13 & 18.44 & 34.04 & 2 & No & B from P3 \\
\hline 14 & 18.17 & 31.99 & 2 & No & B from P3 \\
\hline 15 & 15.93 & 24.91 & 2 & No & B from P3 \\
\hline 16 & 15.55 & 26.68 & 2 & No & B from P3 \\
\hline 17 & 14.83 & 27.16 & 2 & No & B from P3 \\
\hline 18 & 14.79 & 26.47 & 2 & No & B from P3 \\
\hline 19 & 14.08 & 30.78 & 2 & No & B from P3 \\
\hline 20 & 13.66 & 28.37 & 2 & No & B from P3 \\
\hline 21 & 13.17 & 28.32 & 2 & No & B from P3 \\
\hline 22 & 12.93 & 30.0 & 2 & No & B from P3 \\
\hline 23 & 12.34 & 30.59 & 2 & No & B from P3 \\
\hline 24 & 12.09 & 30.64 & 2 & No & B from P3 \\
\hline 25 & 11.36 & 33.1 & 2 & No & B from P3 \\
\hline 26 & 11.28 & 32.17 & 2 & No & B from P3 \\
\hline 27 & 11.11 & 35.35 & 2 & No & B from P3 \\
\hline 28 & 9.66 & 28.64 & 2 & No & B from CCSD(T) \\
\hline
\end{tabular}


Table A26. Molecular orbital data for 2-methyl-2-hexene. Double-ionization threshold $=24.10 \mathrm{eV}$ from CCSD(T)/cc-pV(T+d)Z (to singlet dication).

\begin{tabular}{llllll}
\hline MO & B/eV & U/eV & N & DblIon & Remarks \\
\hline 1 & 305.6 & 436.02 & 2 & Yes & \\
2 & 305.31 & 435.98 & 2 & Yes & \\
3 & 305.28 & 436.1 & 2 & Yes & \\
4 & 305.27 & 436.01 & 2 & Yes & \\
5 & 305.23 & 436.02 & 2 & Yes & \\
6 & 305.14 & 436.04 & 2 & Yes & \\
7 & 305.11 & 436.03 & 2 & Yes & \\
8 & 29.9 & 37.29 & 2 & Yes & B from P3 \\
9 & 28.56 & 35.5 & 2 & Yes & B from P3 \\
10 & 26.23 & 36.46 & 2 & Yes & B from P3 \\
11 & 25.48 & 34.92 & 2 & Yes & \\
12 & 23.84 & 38.18 & 2 & No & B from P3 \\
13 & 19.34 & 33.89 & 2 & No & B from P3 \\
14 & 17.08 & 32.92 & 2 & No & B from P3 \\
15 & 15.83 & 25.32 & 2 & No & B from P3 \\
16 & 14.96 & 28.76 & 2 & No & B from P3 \\
17 & 14.87 & 26.29 & 2 & No & B from P3 \\
18 & 14.66 & 23.93 & 2 & No & B from P3 \\
19 & 13.99 & 27.6 & 2 & No & B from P3 \\
20 & 13.25 & 29.67 & 2 & No & B from P3 \\
21 & 13.32 & 27.07 & 2 & No & B from P3 \\
22 & 13.09 & 30.57 & 2 & No & B from P3 \\
23 & 12.71 & 30.51 & 2 & No & B from P3 \\
24 & 12.14 & 34.15 & 2 & No & B from P3 \\
25 & 11.87 & 31.22 & 2 & No & B from P3 \\
26 & 11.56 & 30.28 & 2 & No & B from P3 \\
27 & 11.14 & 36.2 & 2 & No & B from P3 \\
28 & 8.83 & 30.12 & 2 & No & B from CCSD(T) \\
\hline & & & & & \\
\hline
\end{tabular}

Table A27. Molecular orbital data for 3-methyl-2-hexene. Double-ionization threshold $=24.16 \mathrm{eV}$ from CCSD(T)/cc-pV(T+d)Z (to singlet dication).

\begin{tabular}{llllll}
\hline MO & B/eV & U/eV & N & DblIon & Remarks \\
\hline 1 & 305.52 & 436.0 & 2 & Yes & \\
2 & 305.3 & 435.98 & 2 & Yes & \\
3 & 305.28 & 436.13 & 2 & Yes & \\
4 & 305.24 & 436.0 & 2 & Yes & \\
5 & 305.24 & 435.96 & 2 & Yes & \\
6 & 305.21 & 436.12 & 2 & Yes & \\
7 & 305.12 & 436.03 & 2 & Yes & \\
8 & 29.97 & 36.94 & 2 & Yes & B from P3 \\
9 & 28.27 & 35.95 & 2 & Yes & \\
10 & 26.18 & 35.99 & 2 & Yes & B from P3 \\
11 & 21.81 & 35.38 & 2 & No & B from P2 \\
12 & 23.81 & 37.7 & 2 & No & B from P3 \\
13 & 19.36 & 34.15 & 2 & No & B from P3 \\
14 & 17.01 & 33.23 & 2 & No & B from P3 \\
15 & 15.8 & 25.36 & 2 & No & B from P3 \\
16 & 15.45 & 25.58 & 2 & No & B from P3 \\
17 & 14.39 & 28.44 & 2 & No & B from P3 \\
18 & 14.39 & 25.63 & 2 & No & B from P3 \\
19 & 13.9 & 27.69 & 2 & No & B from P3 \\
20 & 13.75 & 27.08 & 2 & No & B from P3 \\
21 & 13.25 & 29.54 & 2 & No & B from P3 \\
22 & 13.02 & 30.87 & 2 & No & B from P3 \\
23 & 12.78 & 29.44 & 2 & No & B from P3 \\
24 & 12.18 & 33.34 & 2 & No & B from P3 \\
25 & 11.86 & 31.42 & 2 & No & B from P3 \\
26 & 11.55 & 29.48 & 2 & No & B from P3 \\
27 & 11.06 & 37.46 & 2 & No & B from P3 \\
28 & 8.82 & 30.16 & 2 & No & B from CCSD(T) \\
\hline & & & & &
\end{tabular}


Table A28. Molecular orbital data for 4-methyl-2-hexene. Double-ionization threshold = $24.79 \mathrm{eV}$ from P3 (to singlet dication).

\begin{tabular}{llllll}
\hline MO & $\mathbf{B} / \mathbf{e V}$ & $\mathbf{U} / \mathbf{e V}$ & $\mathbf{N}$ & DblIon & Remarks \\
\hline 1 & 305.54 & 436.07 & 2 & Yes & \\
2 & 305.41 & 435.92 & 2 & Yes & \\
3 & 305.3 & 436.03 & 2 & Yes & \\
4 & 305.27 & 436.09 & 2 & Yes & \\
5 & 305.27 & 436.06 & 2 & Yes & \\
6 & 305.11 & 436.03 & 2 & Yes & \\
7 & 305.08 & 436.03 & 2 & Yes & \\
8 & 29.96 & 36.2 & 2 & Yes & \\
9 & 28.37 & 37.15 & 2 & Yes & \\
10 & 26.7 & 35.68 & 2 & Yes & B from P3 \\
11 & 25.16 & 36.81 & 2 & Yes & \\
12 & 23.51 & 35.95 & 2 & No & B from P3 \\
13 & 18.78 & 35.96 & 2 & No & B from P3 \\
14 & 18.04 & 29.08 & 2 & No & B from P3 \\
15 & 15.57 & 27.02 & 2 & No & B from P3 \\
16 & 15.41 & 26.01 & 2 & No & B from P3 \\
17 & 14.58 & 27.64 & 2 & No & B from P3 \\
18 & 14.11 & 29.54 & 2 & No & B from P3 \\
19 & 14.04 & 26.26 & 2 & No & B from P3 \\
20 & 13.76 & 27.69 & 2 & No & B from P3 \\
21 & 13.33 & 28.75 & 2 & No & B from P3 \\
22 & 13.1 & 30.46 & 2 & No & B from P3 \\
23 & 12.55 & 29.57 & 2 & No & B from P3 \\
24 & 12.32 & 31.45 & 2 & No & B from P3 \\
25 & 11.48 & 31.3 & 2 & No & B from P3 \\
26 & 11.37 & 30.87 & 2 & No & B from P3 \\
27 & 11.08 & 37.15 & 2 & No & B from P3 \\
28 & 9.15 & 30.2 & 2 & No & B from CCSD(T) \\
\hline
\end{tabular}

Table A29. Molecular orbital data for 5-methyl-2-hexene. Double-ionization threshold $=24.79 \mathrm{eV}$ from P3 (to singlet dication).

\begin{tabular}{|c|c|c|c|c|c|}
\hline MO & $\mathrm{B} / \mathrm{eV}$ & $\mathrm{U} / \mathrm{eV}$ & $\mathbf{N}$ & DblIon & Remarks \\
\hline 1 & 305.5 & 436.08 & 2 & Yes & \\
\hline 2 & 305.42 & 435.89 & 2 & Yes & \\
\hline 3 & 305.34 & 435.97 & 2 & Yes & \\
\hline 4 & 305.32 & 436.16 & 2 & Yes & \\
\hline 5 & 305.31 & 436.07 & 2 & Yes & \\
\hline 6 & 305.07 & 436.02 & 2 & Yes & \\
\hline 7 & 304.96 & 436.02 & 2 & Yes & \\
\hline 8 & 29.88 & 35.89 & 2 & Yes & \\
\hline 9 & 28.66 & 37.57 & 2 & Yes & \\
\hline 10 & 26.09 & 36.21 & 2 & Yes & B from P3 \\
\hline 11 & 25.15 & 34.66 & 2 & Yes & \\
\hline 12 & 23.36 & 38.23 & 2 & No & B from P3 \\
\hline 13 & 20.86 & 34.5 & 2 & No & B from P3 \\
\hline 14 & 18.03 & 29.88 & 2 & No & B from P3 \\
\hline 15 & 15.61 & 26.32 & 2 & No & B from P3 \\
\hline 16 & 15.05 & 28.47 & 2 & No & B from P3 \\
\hline 17 & 14.8 & 27.06 & 2 & No & B from P3 \\
\hline 18 & 14.55 & 27.44 & 2 & No & B from P3 \\
\hline 19 & 14.09 & 25.44 & 2 & No & B from P3 \\
\hline 20 & 13.68 & 30.54 & 2 & No & B from P3 \\
\hline 21 & 13.3 & 27.1 & 2 & No & B from P3 \\
\hline 22 & 12.94 & 29.05 & 2 & No & B from P3 \\
\hline 23 & 12.56 & 30.66 & 2 & No & B from P3 \\
\hline 24 & 12.04 & 31.23 & 2 & No & B from P3 \\
\hline 25 & 11.6 & 33.84 & 2 & No & B from P3 \\
\hline 26 & 11.35 & 30.4 & 2 & No & B from P3 \\
\hline 27 & 11.2 & 36.53 & 2 & No & B from P3 \\
\hline 28 & 9.17 & 29.75 & 2 & No & B from CCSD(T) \\
\hline
\end{tabular}


Table A30. Molecular orbital data for 2-methyl-3-hexene. Double-ionization threshold $=24.66 \mathrm{eV}$ from CCSD(T)/cc-pV(T+d)Z (to singlet dication).

\begin{tabular}{llllll}
\hline MO & B/eV & U/eV & N & DblIon & Remarks \\
\hline 1 & 305.59 & 436.07 & 2 & Yes & \\
2 & 305.44 & 436.02 & 2 & Yes & \\
3 & 305.35 & 435.86 & 2 & Yes & \\
4 & 305.29 & 436.19 & 2 & Yes & \\
5 & 305.17 & 436.04 & 2 & Yes & \\
6 & 305.11 & 436.03 & 2 & Yes & \\
7 & 305.11 & 436.03 & 2 & Yes & \\
8 & 29.92 & 36.78 & 2 & Yes & B from P3 \\
9 & 28.62 & 35.56 & 2 & Yes & B from P3 \\
10 & 26.48 & 38.36 & 2 & Yes & B from P3 \\
11 & 25.18 & 34.78 & 2 & Yes & \\
12 & 23.16 & 36.14 & 2 & No & B from P3 \\
13 & 21.41 & 36.59 & 2 & No & \\
14 & 18.05 & 28.72 & 2 & No & B from P3 \\
15 & 15.78 & 26.51 & 2 & No & B from P3 \\
16 & 15.19 & 27.66 & 2 & No & B from P3 \\
17 & 14.7 & 26.12 & 2 & No & B from P3 \\
18 & 14.47 & 26.92 & 2 & No & B from P3 \\
19 & 13.95 & 27.71 & 2 & No & B from P3 \\
20 & 13.35 & 27.03 & 2 & No & B from P3 \\
21 & 13.15 & 30.2 & 2 & No & B from P3 \\
22 & 13.09 & 32.08 & 2 & No & B from P3 \\
23 & 12.61 & 30.0 & 2 & No & B from P3 \\
24 & 11.96 & 30.49 & 2 & No & B from P3 \\
25 & 11.9 & 31.8 & 2 & No & B from P3 \\
26 & 11.59 & 30.81 & 2 & No & B from P3 \\
27 & 11.09 & 35.95 & 2 & No & B from P3 \\
28 & 9.14 & 30.52 & 2 & No & B from CCSD(T) \\
\hline & & & & & \\
\hline
\end{tabular}

Table A31. Molecular orbital data for 3-methyl-3-hexene. Double-ionization threshold $=24.17 \mathrm{eV}$ from CCSD(T)/cc-pV(T+d)Z (to singlet dication).

\begin{tabular}{llllll}
\hline MO & B/eV & U/eV & N & DblIon & Remarks \\
\hline 1 & 305.54 & 436.0 & 2 & Yes & \\
2 & 305.37 & 436.03 & 2 & Yes & \\
3 & 305.37 & 436.06 & 2 & Yes & \\
4 & 305.25 & 436.01 & 2 & Yes & \\
5 & 305.15 & 436.04 & 2 & Yes & \\
6 & 305.14 & 436.03 & 2 & Yes & \\
7 & 305.13 & 436.04 & 2 & Yes & \\
8 & 29.98 & 37.18 & 2 & Yes & \\
9 & 28.23 & 35.16 & 2 & Yes & B from P3 \\
10 & 26.74 & 36.44 & 2 & Yes & B from P3 \\
11 & 25.14 & 36.68 & 2 & Yes & B from P3 \\
12 & 23.26 & 35.04 & 2 & No & B from P3 \\
13 & 19.55 & 36.26 & 2 & No & B from P3 \\
14 & 17.07 & 32.2 & 2 & No & B from P3 \\
15 & 15.85 & 25.74 & 2 & No & B from P3 \\
16 & 15.29 & 25.56 & 2 & No & B from P3 \\
17 & 14.6 & 26.25 & 2 & No & B from P3 \\
18 & 14.28 & 27.71 & 2 & No & B from P3 \\
19 & 14.21 & 26.28 & 2 & No & B from P3 \\
20 & 13.47 & 27.68 & 2 & No & B from P3 \\
21 & 13.12 & 31.11 & 2 & No & B from P3 \\
22 & 12.75 & 31.47 & 2 & No & B from P3 \\
23 & 12.51 & 32.02 & 2 & No & B from P3 \\
24 & 12.49 & 30.21 & 2 & No & B from P3 \\
25 & 11.94 & 29.52 & 2 & No & B from P3 \\
26 & 11.74 & 31.63 & 2 & No & B from P3 \\
27 & 10.98 & 36.18 & 2 & No & B from P3 \\
28 & 8.81 & 30.49 & 2 & No & B from CCSD(T) \\
\hline & & & & &
\end{tabular}


Volume 122, Article No. 28 (2017) https://doi.org/10.6028/jres.122.028

Journal of Research of the National Institute of Standards and Technology

Table A32. Molecular orbital data for 2,3-dimethyl-1-pentene. Double-ionization threshold $=24.89 \mathrm{eV}$ from CCSD(T)/cc-pV(T+d)Z (to singlet dication).

\begin{tabular}{|c|c|c|c|c|c|}
\hline MO & B/eV & U/eV & $\mathbf{N}$ & DblIon & Remarks \\
\hline 1 & 305.7 & 436.02 & 2 & Yes & \\
\hline 2 & 305.59 & 436.09 & 2 & Yes & \\
\hline 3 & 305.32 & 436.06 & 2 & Yes & \\
\hline 4 & 305.3 & 436.01 & 2 & Yes & \\
\hline 5 & 305.16 & 436.03 & 2 & Yes & \\
\hline 6 & 305.14 & 436.02 & 2 & Yes & \\
\hline 7 & 304.98 & 436.0 & 2 & Yes & \\
\hline 8 & 30.22 & 36.01 & 2 & Yes & \\
\hline 9 & 28.17 & 37.05 & 2 & Yes & \\
\hline 10 & 26.44 & 35.97 & 2 & Yes & B from P3 \\
\hline 11 & 25.31 & 36.44 & 2 & Yes & \\
\hline 12 & 20.7 & 34.94 & 2 & No & B from P2 \\
\hline 13 & 19.13 & 34.91 & 2 & No & B from P3 \\
\hline 14 & 17.3 & 32.92 & 2 & No & B from P3 \\
\hline 15 & 15.66 & 26.81 & 2 & No & B from P3 \\
\hline 16 & 15.5 & 25.51 & 2 & No & B from P3 \\
\hline 17 & 14.66 & 27.63 & 2 & No & B from P3 \\
\hline 18 & 14.51 & 26.36 & 2 & No & B from P3 \\
\hline 19 & 14.2 & 26.24 & 2 & No & B from P3 \\
\hline 20 & 13.58 & 28.73 & 2 & No & B from P3 \\
\hline 21 & 13.31 & 27.85 & 2 & No & B from P3 \\
\hline 22 & 13.01 & 30.63 & 2 & No & B from P3 \\
\hline 23 & 12.68 & 30.99 & 2 & No & B from P3 \\
\hline 24 & 12.47 & 31.5 & 2 & No & B from P3 \\
\hline 25 & 11.49 & 32.08 & 2 & No & B from P3 \\
\hline 26 & 11.34 & 31.96 & 2 & No & B from P3 \\
\hline 27 & 11.04 & 36.83 & 2 & No & B from P3 \\
\hline 28 & 9.25 & 29.37 & 2 & No & B from CCSD $(\mathrm{T})$ \\
\hline
\end{tabular}


Table A33. Molecular orbital data for 2,4-dimethyl-1-pentene. Double-ionization threshold = $23.04 \mathrm{eV}$ from P3 (to singlet or triplet dication).

\begin{tabular}{|c|c|c|c|c|c|}
\hline MO & $\mathrm{B} / \mathrm{eV}$ & U/eV & $\mathbf{N}$ & DblIon & Remarks \\
\hline 1 & 305.76 & 436.03 & 2 & Yes & \\
\hline 2 & 305.56 & 436.08 & 2 & Yes & \\
\hline 3 & 305.38 & 436.05 & 2 & Yes & \\
\hline 4 & 305.34 & 436.01 & 2 & Yes & \\
\hline 5 & 305.12 & 436.02 & 2 & Yes & \\
\hline 6 & 305.02 & 436.0 & 2 & Yes & \\
\hline 7 & 305.0 & 436.02 & 2 & Yes & \\
\hline 8 & 30.13 & 35.91 & 2 & Yes & \\
\hline 9 & 28.63 & 37.32 & 2 & Yes & B from P3 \\
\hline 10 & 25.42 & 35.73 & 2 & Yes & \\
\hline 11 & 26.0 & 34.69 & 2 & Yes & B from P3 \\
\hline 12 & 25.01 & 37.41 & 2 & Yes & B from P3 \\
\hline 13 & 18.77 & 34.33 & 2 & No & B from P3 \\
\hline 14 & 17.4 & 33.29 & 2 & No & B from P3 \\
\hline 15 & 15.74 & 26.22 & 2 & No & B from P3 \\
\hline 16 & 15.27 & 26.26 & 2 & No & B from P3 \\
\hline 17 & 14.94 & 25.76 & 2 & No & B from P3 \\
\hline 18 & 14.59 & 28.49 & 2 & No & B from P3 \\
\hline 19 & 14.07 & 27.04 & 2 & No & B from P3 \\
\hline 20 & 13.78 & 27.57 & 2 & No & B from P3 \\
\hline 21 & 13.35 & 27.02 & 2 & No & B from P3 \\
\hline 22 & 12.88 & 29.73 & 2 & No & B from P3 \\
\hline 23 & 12.63 & 31.02 & 2 & No & B from P3 \\
\hline 24 & 12.12 & 32.7 & 2 & No & B from P3 \\
\hline 25 & 11.61 & 33.68 & 2 & No & B from P3 \\
\hline 26 & 11.38 & 30.84 & 2 & No & B from P3 \\
\hline 27 & 11.17 & 36.72 & 2 & No & B from P3 \\
\hline 28 & 9.27 & 29.02 & 2 & No & B from $\operatorname{CCSD}(\mathrm{T})$ \\
\hline
\end{tabular}

Table A34. Molecular orbital data for 3,4-dimethyl-1-pentene. Double-ionization threshold = 25.24 eV from EPT (to singlet dication).

\begin{tabular}{|c|c|c|c|c|c|}
\hline MO & $\mathrm{B} / \mathrm{eV}$ & U/eV & $\mathbf{N}$ & DblIon & Remarks \\
\hline 1 & 305.6 & 436.03 & 2 & Yes & \\
\hline 2 & 305.53 & 436.01 & 2 & Yes & \\
\hline 3 & 305.53 & 436.12 & 2 & Yes & \\
\hline 4 & 305.24 & 436.03 & 2 & Yes & \\
\hline 5 & 305.14 & 436.02 & 2 & Yes & \\
\hline 6 & 305.1 & 436.02 & 2 & Yes & \\
\hline 7 & 305.09 & 436.02 & 2 & Yes & \\
\hline 8 & 30.17 & 35.15 & 2 & Yes & \\
\hline 9 & 28.2 & 38.16 & 2 & Yes & \\
\hline 10 & 26.59 & 37.29 & 2 & Yes & B from P3 \\
\hline 11 & 25.24 & 34.58 & 2 & Yes & \\
\hline 12 & 20.77 & 35.27 & 2 & No & B from P3 \\
\hline 13 & 18.81 & 34.97 & 2 & No & B from P3 \\
\hline 14 & 17.83 & 31.44 & 2 & No & B from P3 \\
\hline 15 & 15.95 & 25.05 & 2 & No & B from P3 \\
\hline 16 & 15.19 & 29.64 & 2 & No & B from P3 \\
\hline 17 & 15.01 & 26.29 & 2 & No & B from P3 \\
\hline 18 & 14.7 & 27.15 & 2 & No & B from P3 \\
\hline 19 & 13.84 & 26.71 & 2 & No & B from P3 \\
\hline 20 & 13.44 & 27.51 & 2 & No & B from P3 \\
\hline 21 & 13.24 & 30.26 & 2 & No & B from P3 \\
\hline 22 & 13.01 & 29.21 & 2 & No & B from P3 \\
\hline 23 & 12.82 & 30.57 & 2 & No & B from P3 \\
\hline 24 & 12.18 & 30.62 & 2 & No & B from P3 \\
\hline 25 & 11.22 & 33.56 & 2 & No & B from P3 \\
\hline 26 & 11.24 & 31.91 & 2 & No & B from P3 \\
\hline 27 & 11.04 & 36.09 & 2 & No & B from P3 \\
\hline 28 & 9.62 & 29.11 & 2 & No & B from CCSD(T) \\
\hline
\end{tabular}


Table A35. Molecular orbital data for 3,3-dimethyl-1-pentene. Double-ionization threshold = $24.60 \mathrm{eV}$ from CCSD(T)/cc-pV(T+d)Z (to triplet dication).

\begin{tabular}{|c|c|c|c|c|c|}
\hline MO & $\mathrm{B} / \mathrm{eV}$ & $\mathrm{U} / \mathrm{eV}$ & $\mathbf{N}$ & DblIon & Remarks \\
\hline 1 & 305.86 & 436.12 & 2 & Yes & \\
\hline 2 & 305.52 & 435.99 & 2 & Yes & \\
\hline 3 & 305.34 & 436.06 & 2 & Yes & \\
\hline 4 & 305.19 & 436.02 & 2 & Yes & \\
\hline 5 & 305.14 & 436.03 & 2 & Yes & \\
\hline 6 & 305.13 & 436.02 & 2 & Yes & \\
\hline 7 & 305.08 & 436.01 & 2 & Yes & \\
\hline 8 & 30.39 & 35.24 & 2 & Yes & \\
\hline 9 & 27.76 & 38.19 & 2 & Yes & B from P3 \\
\hline 10 & 26.69 & 37.28 & 2 & Yes & B from P3 \\
\hline 11 & 25.34 & 34.33 & 2 & Yes & B from P3 \\
\hline 12 & 23.77 & 34.12 & 2 & No & \\
\hline 13 & 21.57 & 35.96 & 2 & No & \\
\hline 14 & 17.33 & 33.11 & 2 & No & B from P3 \\
\hline 15 & 15.89 & 24.85 & 2 & No & B from P3 \\
\hline 16 & 15.44 & 26.78 & 2 & No & B from P3 \\
\hline 17 & 14.93 & 27.83 & 2 & No & B from P3 \\
\hline 18 & 14.84 & 26.67 & 2 & No & B from P3 \\
\hline 19 & 13.71 & 27.8 & 2 & No & B from P3 \\
\hline 20 & 13.4 & 28.83 & 2 & No & B from P3 \\
\hline 21 & 13.33 & 28.26 & 2 & No & B from P3 \\
\hline 22 & 13.06 & 28.23 & 2 & No & B from P3 \\
\hline 23 & 12.72 & 31.08 & 2 & No & B from P3 \\
\hline 24 & 12.18 & 30.04 & 2 & No & B from P3 \\
\hline 25 & 11.36 & 33.01 & 2 & No & B from P3 \\
\hline 26 & 11.15 & 34.69 & 2 & No & B from P3 \\
\hline 27 & 11.13 & 35.41 & 2 & No & B from P3 \\
\hline 28 & 9.62 & 28.87 & 2 & No & B from CCSD(T) \\
\hline
\end{tabular}

Table A36. Molecular orbital data for 4,4-dimethyl-1-pentene. Double-ionization threshold = 25.17 eV from EPT (to triplet dication).

\begin{tabular}{|c|c|c|c|c|c|}
\hline MO & $\mathrm{B} / \mathrm{eV}$ & $\mathrm{U} / \mathrm{eV}$ & $\mathbf{N}$ & DblIon & Remarks \\
\hline 1 & 305.79 & 436.13 & 2 & Yes & \\
\hline 2 & 305.6 & 435.98 & 2 & Yes & \\
\hline 3 & 305.41 & 436.06 & 2 & Yes & \\
\hline 4 & 305.26 & 436.03 & 2 & Yes & \\
\hline 5 & 305.1 & 436.02 & 2 & Yes & \\
\hline 6 & 305.09 & 436.02 & 2 & Yes & \\
\hline 7 & 304.98 & 436.01 & 2 & Yes & \\
\hline 8 & 30.28 & 34.75 & 2 & Yes & B from P3 \\
\hline 9 & 28.41 & 39.32 & 2 & Yes & \\
\hline 10 & 24.86 & 34.45 & 2 & No & B from P3 \\
\hline 11 & 25.24 & 34.5 & 2 & No & B from P3 \\
\hline 12 & 25.07 & 37.32 & 2 & No & \\
\hline 13 & 18.55 & 34.48 & 2 & No & B from P3 \\
\hline 14 & 17.62 & 33.13 & 2 & No & B from P3 \\
\hline 15 & 15.8 & 26.47 & 2 & No & B from P3 \\
\hline 16 & 15.28 & 25.05 & 2 & No & B from P3 \\
\hline 17 & 15.04 & 27.74 & 2 & No & B from P3 \\
\hline 18 & 14.75 & 27.72 & 2 & No & B from P3 \\
\hline 19 & 14.12 & 28.25 & 2 & No & B from P3 \\
\hline 20 & 13.59 & 27.4 & 2 & No & B from P3 \\
\hline 21 & 13.21 & 28.73 & 2 & No & B from P3 \\
\hline 22 & 12.75 & 28.31 & 2 & No & B from P3 \\
\hline 23 & 12.62 & 29.6 & 2 & No & B from P3 \\
\hline 24 & 12.21 & 30.46 & 2 & No & B from P3 \\
\hline 25 & 11.47 & 33.59 & 2 & No & B from P3 \\
\hline 26 & 11.23 & 34.59 & 2 & No & B from P3 \\
\hline 27 & 11.07 & 36.0 & 2 & No & B from P3 \\
\hline 28 & 9.63 & 28.73 & 2 & No & B from $\operatorname{CCSD}(\mathrm{T})$ \\
\hline
\end{tabular}


Table A37. Molecular orbital data for 2,3-dimethyl-2-pentene. Double-ionization threshold $=23.57 \mathrm{eV}$ from CCSD $(\mathrm{T}) / \mathrm{cc}-\mathrm{pV}(\mathrm{T}+\mathrm{d}) \mathrm{Z}$ (to singlet dication).

\begin{tabular}{|c|c|c|c|c|c|}
\hline MO & $\mathrm{B} / \mathrm{eV}$ & $\mathrm{U} / \mathrm{eV}$ & $\mathbf{N}$ & DblIon & Remarks \\
\hline 1 & 305.45 & 435.9 & 2 & Yes & \\
\hline 2 & 305.38 & 436.15 & 2 & Yes & \\
\hline 3 & 305.31 & 436.06 & 2 & Yes & \\
\hline 4 & 305.17 & 436.01 & 2 & Yes & \\
\hline 5 & 305.17 & 436.02 & 2 & Yes & \\
\hline 6 & 305.17 & 436.02 & 2 & Yes & \\
\hline 7 & 305.13 & 436.04 & 2 & Yes & \\
\hline 8 & 30.11 & 37.11 & 2 & Yes & \\
\hline 9 & 27.91 & 35.56 & 2 & Yes & B from P3 \\
\hline 10 & 26.52 & 35.26 & 2 & Yes & \\
\hline 11 & 25.31 & 35.29 & 2 & Yes & B from P3 \\
\hline 12 & 24.44 & 36.89 & 2 & Yes & B from P3 \\
\hline 13 & 22.46 & 36.25 & 2 & No & \\
\hline 14 & 16.34 & 34.65 & 2 & No & B from P3 \\
\hline 15 & 15.88 & 25.42 & 2 & No & B from P3 \\
\hline 16 & 15.01 & 26.46 & 2 & No & B from P3 \\
\hline 17 & 14.67 & 24.22 & 2 & No & B from P3 \\
\hline 18 & 14.13 & 26.93 & 2 & No & B from P3 \\
\hline 19 & 13.99 & 27.24 & 2 & No & B from P3 \\
\hline 20 & 13.56 & 27.11 & 2 & No & B from P3 \\
\hline 21 & 13.16 & 27.83 & 2 & No & B from P3 \\
\hline 22 & 13.06 & 29.11 & 2 & No & B from P3 \\
\hline 23 & 12.6 & 30.78 & 2 & No & B from P3 \\
\hline 24 & 12.55 & 31.57 & 2 & No & B from P3 \\
\hline 25 & 12.27 & 33.35 & 2 & No & B from P3 \\
\hline 26 & 11.76 & 31.51 & 2 & No & B from P3 \\
\hline 27 & 10.84 & 37.52 & 2 & No & B from P3 \\
\hline 28 & 8.5 & 30.75 & 2 & No & B from CCSD $(\mathrm{T})$ \\
\hline
\end{tabular}

Table A38. Molecular orbital data for 2,4-dimethyl-2-pentene. Double-ionization threshold $=28.98 \mathrm{eV}$ from CCSD $(\mathrm{T}) / \mathrm{cc}-\mathrm{pV}(\mathrm{T}+\mathrm{d}) \mathrm{Z}$ (to singlet dication).

\begin{tabular}{llllll}
\hline MO & B/eV & U/eV & N & DblIon & Remarks \\
\hline 1 & 305.61 & 436.02 & 2 & Yes & \\
2 & 305.53 & 436.07 & 2 & Yes & \\
3 & 305.28 & 436.01 & 2 & Yes & \\
4 & 305.24 & 436.02 & 2 & Yes & \\
5 & 305.11 & 436.03 & 2 & Yes & \\
6 & 305.07 & 436.02 & 2 & Yes & \\
7 & 305.07 & 436.04 & 2 & Yes & \\
8 & 30.05 & 36.96 & 2 & Yes & B from P3 \\
9 & 28.5 & 36.0 & 2 & No & B from P3 \\
10 & 24.84 & 34.98 & 2 & No & B from P3 \\
11 & 22.14 & 34.77 & 2 & No & B from P3 \\
12 & 25.0 & 37.87 & 2 & No & B from P3 \\
13 & 19.09 & 36.37 & 2 & No & B from P3 \\
14 & 17.21 & 31.0 & 2 & No & B from P3 \\
15 & 15.65 & 28.35 & 2 & No & B from P3 \\
16 & 14.95 & 27.47 & 2 & No & B from P3 \\
17 & 14.69 & 23.84 & 2 & No & B from P3 \\
18 & 14.38 & 27.0 & 2 & No & B from P3 \\
19 & 13.81 & 27.7 & 2 & No & B from P3 \\
20 & 13.64 & 28.71 & 2 & No & B from P3 \\
21 & 13.32 & 26.9 & 2 & No & B from P3 \\
22 & 13.29 & 26.87 & 2 & No & B from P3 \\
23 & 12.77 & 31.01 & 2 & No & B from P3 \\
24 & 12.29 & 33.33 & 2 & No & B from P3 \\
25 & 11.84 & 32.72 & 2 & No & B from P3 \\
26 & 11.53 & 33.41 & 2 & No & B from P3 \\
27 & 11.0 & 35.1 & 2 & No & B from P3 \\
28 & 8.83 & 30.47 & 2 & No & B from CCSD(T) \\
\hline & & & & & \\
\hline
\end{tabular}


Table A39. Molecular orbital data for 3,4-dimethyl-2-pentene. Double-ionization threshold $=29.07 \mathrm{eV}$ from CCSD(T)/cc-pV(T+d)Z (to singlet dication).

\begin{tabular}{|c|c|c|c|c|c|}
\hline MO & $\mathrm{B} / \mathrm{eV}$ & $\mathrm{U} / \mathrm{eV}$ & $\mathbf{N}$ & DblIon & Remarks \\
\hline 1 & 305.54 & 436.05 & 2 & Yes & \\
\hline 2 & 305.49 & 436.04 & 2 & Yes & \\
\hline 3 & 305.24 & 435.95 & 2 & Yes & \\
\hline 4 & 305.22 & 436.01 & 2 & Yes & \\
\hline 5 & 305.21 & 436.11 & 2 & Yes & \\
\hline 6 & 305.08 & 436.02 & 2 & Yes & \\
\hline 7 & 305.08 & 436.03 & 2 & Yes & \\
\hline 8 & 30.13 & 36.55 & 2 & Yes & B from P3 \\
\hline 9 & 28.06 & 36.46 & 2 & No & \\
\hline 10 & 27.69 & 36.13 & 2 & No & B from P3 \\
\hline 11 & 21.95 & 34.7 & 2 & No & $\mathrm{B}$ from P2 \\
\hline 12 & 24.48 & 35.98 & 2 & No & B from P3 \\
\hline 13 & 21.57 & 36.92 & 2 & No & B from P3 \\
\hline 14 & 17.0 & 32.24 & 2 & No & B from P3 \\
\hline 15 & 15.97 & 25.13 & 2 & No & B from P3 \\
\hline 16 & 14.93 & 27.69 & 2 & No & B from P3 \\
\hline 17 & 14.48 & 24.7 & 2 & No & B from P3 \\
\hline 18 & 13.92 & 29.4 & 2 & No & B from P3 \\
\hline 19 & 13.74 & 30.4 & 2 & No & B from P3 \\
\hline 20 & 13.86 & 25.45 & 2 & No & B from P3 \\
\hline 21 & 13.74 & 27.3 & 2 & No & B from P3 \\
\hline 22 & 13.1 & 29.02 & 2 & No & B from P3 \\
\hline 23 & 13.03 & 27.92 & 2 & No & B from P3 \\
\hline 24 & 12.24 & 32.06 & 2 & No & B from P3 \\
\hline 25 & 11.8 & 33.0 & 2 & No & B from P3 \\
\hline 26 & 11.44 & 32.89 & 2 & No & B from P3 \\
\hline 27 & 10.94 & 36.42 & 2 & No & B from P3 \\
\hline 28 & 8.82 & 30.41 & 2 & No & B from CCSD(T) \\
\hline
\end{tabular}


Table A40. Molecular orbital data for 4,4-dimethyl-2-pentene. Double-ionization threshold $=24.71 \mathrm{eV}$ from CCSD(T)/cc-pV(T+d)Z (to singlet dication).

\begin{tabular}{|c|c|c|c|c|c|}
\hline MO & $\mathrm{B} / \mathrm{eV}$ & $\mathrm{U} / \mathrm{eV}$ & $\mathbf{N}$ & DblIon & Remarks \\
\hline 1 & 305.82 & 436.12 & 2 & Yes & \\
\hline 2 & 305.38 & 435.9 & 2 & Yes & \\
\hline 3 & 305.29 & 436.03 & 2 & Yes & \\
\hline 4 & 305.26 & 436.11 & 2 & Yes & \\
\hline 5 & 305.09 & 436.02 & 2 & Yes & \\
\hline 6 & 305.09 & 436.03 & 2 & Yes & \\
\hline 7 & 305.06 & 436.01 & 2 & Yes & \\
\hline 8 & 30.27 & 35.81 & 2 & Yes & B from P3 \\
\hline 9 & 28.31 & 37.75 & 2 & Yes & B from P3 \\
\hline 10 & 25.29 & 34.33 & 2 & Yes & \\
\hline 11 & 25.21 & 34.57 & 2 & Yes & \\
\hline 12 & 25.11 & 37.65 & 2 & Yes & \\
\hline 13 & 21.33 & 36.18 & 2 & No & B from P3 \\
\hline 14 & 17.33 & 31.54 & 2 & No & B from P3 \\
\hline 15 & 15.7 & 26.81 & 2 & No & B from P3 \\
\hline 16 & 15.06 & 28.15 & 2 & No & B from P3 \\
\hline 17 & 14.81 & 25.68 & 2 & No & B from P3 \\
\hline 18 & 14.0 & 28.91 & 2 & No & B from P3 \\
\hline 19 & 14.07 & 25.17 & 2 & No & B from P3 \\
\hline 20 & 13.79 & 29.17 & 2 & No & B from P3 \\
\hline 21 & 13.44 & 26.71 & 2 & No & B from P3 \\
\hline 22 & 13.04 & 29.15 & 2 & No & B from P3 \\
\hline 23 & 12.73 & 28.35 & 2 & No & B from P3 \\
\hline 24 & 12.44 & 30.69 & 2 & No & B from P3 \\
\hline 25 & 11.57 & 33.18 & 2 & No & B from P3 \\
\hline 26 & 11.29 & 35.62 & 2 & No & B from P3 \\
\hline 27 & 11.11 & 35.45 & 2 & No & B from P3 \\
\hline 28 & 9.16 & 29.87 & 2 & No & B from CCSD $(\mathrm{T})$ \\
\hline
\end{tabular}

Table A41. Molecular orbital data for 2-ethyl-1-pentene. Double-ionization threshold = 25.29 eV from EPT (to singlet dication).

\begin{tabular}{|c|c|c|c|c|c|}
\hline MO & $\mathrm{B} / \mathrm{eV}$ & U/eV & $\mathbf{N}$ & DblIon & Remarks \\
\hline 1 & 305.68 & 436.02 & 2 & Yes & \\
\hline 2 & 305.46 & 436.05 & 2 & Yes & \\
\hline 3 & 305.39 & 436.0 & 2 & Yes & \\
\hline 4 & 305.36 & 436.1 & 2 & Yes & \\
\hline 5 & 305.21 & 436.04 & 2 & Yes & \\
\hline 6 & 305.17 & 436.03 & 2 & Yes & \\
\hline 7 & 305.01 & 436.0 & 2 & Yes & \\
\hline 8 & 30.06 & 36.53 & 2 & Yes & \\
\hline 9 & 28.32 & 35.51 & 2 & Yes & B from P3 \\
\hline 10 & 26.92 & 37.36 & 2 & Yes & \\
\hline 11 & 21.53 & 36.92 & 2 & No & B from P2 \\
\hline 12 & 23.11 & 35.17 & 2 & No & B from P3 \\
\hline 13 & 19.62 & 33.85 & 2 & No & B from P3 \\
\hline 14 & 17.3 & 33.48 & 2 & No & B from P3 \\
\hline 15 & 15.93 & 24.26 & 2 & No & B from P3 \\
\hline 16 & 15.56 & 25.78 & 2 & No & B from P3 \\
\hline 17 & 14.84 & 26.95 & 2 & No & B from P3 \\
\hline 18 & 14.45 & 27.47 & 2 & No & B from P3 \\
\hline 19 & 13.97 & 27.64 & 2 & No & B from P3 \\
\hline 20 & 13.84 & 29.81 & 2 & No & B from P3 \\
\hline 21 & 13.16 & 28.58 & 2 & No & B from P3 \\
\hline 22 & 12.78 & 30.42 & 2 & No & B from P3 \\
\hline 23 & 12.38 & 33.49 & 2 & No & B from P3 \\
\hline 24 & 12.09 & 29.29 & 2 & No & B from P3 \\
\hline 25 & 11.93 & 32.22 & 2 & No & B from P3 \\
\hline 26 & 11.54 & 30.61 & 2 & No & B from P3 \\
\hline 27 & 11.13 & 35.69 & 2 & No & B from P3 \\
\hline 28 & 9.22 & 29.68 & 2 & No & B from CCSD(T) \\
\hline
\end{tabular}


Table A42. Molecular orbital data for 3-ethyl-1-pentene. Double-ionization threshold $=25.28 \mathrm{eV}$ from P3 (to singlet dication).

\begin{tabular}{llllll}
\hline MO & B/eV & U/eV & N & DblIon & Remarks \\
\hline 1 & 305.57 & 436.03 & 2 & Yes & \\
2 & 305.52 & 436.02 & 2 & Yes & \\
3 & 305.32 & 436.05 & 2 & Yes & \\
4 & 305.32 & 436.06 & 2 & Yes & \\
5 & 305.23 & 436.03 & 2 & Yes & \\
6 & 305.15 & 436.03 & 2 & Yes & \\
7 & 305.13 & 436.03 & 2 & Yes & \\
8 & 30.07 & 35.37 & 2 & Yes & B from P3 \\
9 & 27.81 & 38.82 & 2 & Yes & \\
10 & 27.49 & 35.54 & 2 & Yes & B from P3 \\
11 & 21.53 & 37.03 & 2 & No & B from P2 \\
12 & 20.44 & 33.23 & 2 & No & B from P3 \\
13 & 21.52 & 35.79 & 2 & No & B from P3 \\
14 & 17.9 & 31.11 & 2 & No & B from P3 \\
15 & 15.93 & 25.25 & 2 & No & B from P3 \\
16 & 15.44 & 26.63 & 2 & No & B from P3 \\
17 & 15.08 & 26.3 & 2 & No & B from P3 \\
18 & 14.23 & 30.49 & 2 & No & B from P3 \\
19 & 14.02 & 27.46 & 2 & No & B from P3 \\
20 & 13.96 & 27.96 & 2 & No & B from P3 \\
21 & 12.94 & 31.25 & 2 & No & B from P3 \\
22 & 12.78 & 29.7 & 2 & No & B from P3 \\
23 & 12.59 & 28.44 & 2 & No & B from P3 \\
24 & 12.23 & 31.31 & 2 & No & B from P3 \\
25 & 11.35 & 31.79 & 2 & No & B from P3 \\
26 & 11.2 & 32.71 & 2 & No & B from P3 \\
27 & 11.03 & 35.34 & 2 & No & B from P3 \\
28 & 9.62 & 29.07 & 2 & No & B from CCSD(T) \\
\hline & & & & &
\end{tabular}

Table A43. Molecular orbital data for 3-ethyl-2-pentene. Double-ionization threshold $=24.20 \mathrm{eV}$ from CCSD(T)/cc-pV(T+d)Z (to singlet dication).

\begin{tabular}{llllll}
\hline MO & $\mathbf{B} / \mathbf{e V}$ & $\mathbf{U} / \mathbf{e V}$ & $\mathbf{N}$ & DblIon & Remarks \\
\hline 1 & 305.45 & 435.95 & 2 & Yes & \\
2 & 305.38 & 436.06 & 2 & Yes & \\
3 & 305.36 & 436.07 & 2 & Yes & \\
4 & 305.24 & 435.96 & 2 & Yes & \\
5 & 305.21 & 436.11 & 2 & Yes & \\
6 & 305.17 & 436.04 & 2 & Yes & \\
7 & 305.12 & 436.04 & 2 & Yes & \\
8 & 30.04 & 36.88 & 2 & Yes & B from P3 \\
9 & 27.58 & 35.32 & 2 & Yes & B from P3 \\
10 & 27.47 & 36.3 & 2 & Yes & \\
11 & 25.09 & 37.14 & 2 & Yes & \\
12 & 23.05 & 34.02 & 2 & No & \\
13 & 19.58 & 36.58 & 2 & No & B from P2 \\
14 & 16.91 & 32.66 & 2 & No & B from P3 \\
15 & 15.93 & 24.93 & 2 & No & B from P3 \\
16 & 15.25 & 26.51 & 2 & No & B from P3 \\
17 & 14.48 & 26.72 & 2 & No & B from P3 \\
18 & 14.28 & 26.92 & 2 & No & B from P3 \\
19 & 13.97 & 27.5 & 2 & No & B from P3 \\
20 & 13.74 & 28.54 & 2 & No & B from P3 \\
21 & 13.25 & 28.65 & 2 & No & B from P3 \\
22 & 12.82 & 30.23 & 2 & No & B from P3 \\
23 & 12.44 & 32.62 & 2 & No & B from P3 \\
24 & 12.44 & 31.43 & 2 & No & B from P3 \\
25 & 12.09 & 29.07 & 2 & No & B from P3 \\
26 & 11.64 & 32.18 & 2 & No & B from P3 \\
27 & 10.92 & 35.76 & 2 & No & B from P3 \\
28 & 8.78 & 30.78 & 2 & No & B from CCSD(T) \\
\hline & & & & &
\end{tabular}


Table A44. Molecular orbital data for 2,3,3-trimethyl-1-butene. Double-ionization threshold $=25.18 \mathrm{eV}$ from CCSD(T)/cc-pV(T+d)Z (to singlet dication).

\begin{tabular}{llllll}
\hline MO & B/eV & U/eV & N & DblIon & Remarks \\
\hline 1 & 305.92 & 436.13 & 2 & Yes & \\
2 & 305.72 & 436.05 & 2 & Yes & \\
3 & 305.27 & 436.01 & 2 & Yes & \\
4 & 305.14 & 436.02 & 2 & Yes & \\
5 & 305.14 & 436.02 & 2 & Yes & \\
6 & 305.06 & 436.01 & 2 & Yes & \\
7 & 304.96 & 435.99 & 2 & Yes & \\
8 & 30.47 & 35.59 & 2 & Yes & B from P3 \\
9 & 28.0 & 37.93 & 2 & Yes & \\
10 & 25.69 & 34.22 & 2 & Yes & B from P3 \\
11 & 21.93 & 34.49 & 2 & No & B from P2 \\
12 & 24.75 & 37.39 & 2 & No & B from P3 \\
13 & 21.83 & 35.01 & 2 & No & B from P3 \\
14 & 16.73 & 35.42 & 2 & No & B from P3 \\
15 & 15.93 & 25.45 & 2 & No & B from P3 \\
16 & 15.05 & 27.61 & 2 & No & B from P3 \\
17 & 14.87 & 25.79 & 2 & No & B from P3 \\
18 & 14.29 & 26.69 & 2 & No & B from P3 \\
19 & 14.36 & 25.09 & 2 & No & B from P3 \\
20 & 13.73 & 27.64 & 2 & No & B from P3 \\
21 & 13.15 & 27.89 & 2 & No & B from P3 \\
22 & 13.03 & 30.78 & 2 & No & B from P3 \\
23 & 12.72 & 29.06 & 2 & No & B from P3 \\
24 & 12.64 & 30.0 & 2 & No & B from P3 \\
25 & 11.58 & 33.42 & 2 & No & B from P3 \\
26 & 11.26 & 36.06 & 2 & No & B from P3 \\
27 & 11.06 & 36.12 & 2 & No & B from P3 \\
28 & 9.28 & 29.0 & 2 & No & B from CCSD(T) \\
\hline & & & & & \\
\hline
\end{tabular}

Table A45. Molecular orbital data for 2-ethyl-3-methyl-1-butene. Double-ionization threshold $=25.21 \mathrm{eV}$ from CCSD(T)/cc$\mathrm{pV}(\mathrm{T}+\mathrm{d}) \mathrm{Z}$ (to singlet dication).

\begin{tabular}{llllll}
\hline MO & B/eV & U/eV & N & DblIon & Remarks \\
\hline 1 & 305.66 & 435.96 & 2 & Yes & \\
2 & 305.64 & 436.16 & 2 & Yes & \\
3 & 305.45 & 436.05 & 2 & Yes & \\
4 & 305.21 & 436.03 & 2 & Yes & \\
5 & 305.18 & 436.02 & 2 & Yes & \\
6 & 305.12 & 436.03 & 2 & Yes & \\
7 & 305.0 & 436.0 & 2 & Yes & \\
8 & 30.19 & 36.21 & 2 & Yes & \\
9 & 28.07 & 35.91 & 2 & Yes & B from P3 \\
10 & 26.75 & 37.89 & 2 & Yes & B from P3 \\
11 & 22.02 & 34.61 & 2 & No & B from P2 \\
12 & 20.45 & 35.77 & 2 & No & B from P3 \\
13 & 19.62 & 34.97 & 2 & No & B from P3 \\
14 & 17.24 & 32.18 & 2 & No & B from P3 \\
15 & 15.85 & 25.96 & 2 & No & B from P3 \\
16 & 15.34 & 27.02 & 2 & No & B from P3 \\
17 & 14.52 & 27.04 & 2 & No & B from P3 \\
18 & 14.38 & 27.76 & 2 & No & B from P3 \\
19 & 14.13 & 27.04 & 2 & No & B from P3 \\
20 & 13.76 & 27.57 & 2 & No & B from P3 \\
21 & 13.41 & 27.02 & 2 & No & B from P3 \\
22 & 12.92 & 29.13 & 2 & No & B from P3 \\
23 & 12.7 & 32.0 & 2 & No & B from P3 \\
24 & 11.91 & 31.06 & 2 & No & B from P3 \\
25 & 11.89 & 32.74 & 2 & No & B from P3 \\
26 & 11.53 & 34.52 & 2 & No & B from P3 \\
27 & 10.93 & 34.41 & 2 & No & B from P3 \\
28 & 9.21 & 29.82 & 2 & No & B from CCSD(T) \\
\hline & & & & & \\
\hline
\end{tabular}


Table A46. Molecular orbital data for benzaldehyde. Double-ionization threshold $=24.12 \mathrm{eV}$ from CCSD(T)/cc-pV(T+d)Z (to triplet dication).

\begin{tabular}{llllll}
\hline MO & B/eV & U/eV & N & DblIon & Remarks \\
\hline 1 & 559.21 & 794.65 & 2 & Yes & \\
2 & 308.48 & 436.17 & 2 & Yes & \\
3 & 306.45 & 435.95 & 2 & Yes & \\
4 & 306.38 & 435.96 & 2 & Yes & \\
5 & 306.26 & 435.85 & 2 & Yes & \\
6 & 306.21 & 436.07 & 2 & Yes & \\
7 & 306.18 & 436.03 & 2 & Yes & \\
8 & 306.13 & 436.06 & 2 & Yes & \\
9 & 37.9 & 72.05 & 2 & Yes & B from P3 \\
10 & 31.97 & 39.85 & 2 & Yes & \\
11 & 28.66 & 43.11 & 2 & Yes & \\
12 & 28.22 & 42.39 & 2 & Yes & B from P3 \\
13 & 20.32 & 43.42 & 2 & No & B from P2 \\
14 & 20.18 & 38.48 & 2 & No & B from P3 \\
15 & 19.06 & 41.49 & 2 & No & B from P3 \\
16 & 17.5 & 30.14 & 2 & No & B from P3 \\
17 & 16.42 & 38.79 & 2 & No & B from P3 \\
18 & 15.77 & 47.6 & 2 & No & B from P3 \\
19 & 15.07 & 37.39 & 2 & No & B from P3 \\
20 & 14.87 & 48.74 & 2 & No & B from P3 \\
21 & 14.84 & 32.75 & 2 & No & B from P3 \\
22 & 13.8 & 34.99 & 2 & No & B from P3 \\
23 & 13.0 & 40.64 & 2 & No & B from P3 \\
24 & 12.6 & 38.08 & 2 & No & B from P3 \\
25 & 12.49 & 34.29 & 2 & No & B from P3 \\
26 & 9.9 & 57.18 & 2 & No & B from P3 \\
27 & 9.76 & 29.08 & 2 & No & B from P3 \\
28 & 9.8 & 30.46 & 2 & No & B from expt \\
\hline & & & & & \\
\hline
\end{tabular}

Table A47. Molecular orbital data for phenol. Double-ionization threshold $=23.50 \mathrm{eV}$ from CCSD(T)/cc-pV(T+d)Z (to singlet dication).

\begin{tabular}{llllll}
\hline MO & B/eV & U/eV & N & DblIon & Remarks \\
\hline 1 & 560.12 & 794.57 & 2 & Yes & \\
2 & 307.63 & 436.12 & 2 & Yes & \\
3 & 305.96 & 435.97 & 2 & Yes & \\
4 & 305.89 & 435.97 & 2 & Yes & \\
5 & 305.73 & 435.98 & 2 & Yes & \\
6 & 305.55 & 435.97 & 2 & Yes & \\
7 & 305.53 & 436.0 & 2 & Yes & \\
8 & 38.31 & 69.03 & 2 & Yes & \\
9 & 31.3 & 40.79 & 2 & Yes & \\
10 & 27.85 & 42.11 & 2 & Yes & \\
11 & 27.18 & 45.78 & 2 & Yes & B from P3 \\
12 & 20.28 & 39.74 & 2 & No & B from P3 \\
13 & 22.47 & 43.11 & 2 & No & \\
14 & 18.05 & 39.72 & 2 & No & B from P3 \\
15 & 16.7 & 34.83 & 2 & No & B from P3 \\
16 & 15.83 & 42.58 & 2 & No & B from P3 \\
17 & 14.97 & 36.91 & 2 & No & B from P3 \\
18 & 14.53 & 32.83 & 2 & No & B from P3 \\
19 & 13.93 & 47.84 & 2 & No & B from P3 \\
20 & 13.44 & 51.94 & 2 & No & B from expt \\
21 & 12.61 & 39.77 & 2 & No & B from expt \\
22 & 12.02 & 40.14 & 2 & No & B from expt \\
23 & 11.59 & 32.63 & 2 & No & B from expt \\
24 & 9.39 & 28.19 & 2 & No & B from expt \\
25 & 8.7 & 34.75 & 2 & No & B from expt \\
\hline & & & & &
\end{tabular}


Table A48. Molecular orbital data for acetophenone. Double-ionization threshold $=23.42 \mathrm{eV}$ from CCSD(T)/cc-pV(T+d)Z (to triplet dication).

\begin{tabular}{llllll}
\hline MO & B/eV & U/eV & N & DblIon & Remarks \\
\hline 1 & 558.9 & 794.64 & 2 & Yes & \\
2 & 308.5 & 436.2 & 2 & Yes & \\
3 & 306.29 & 435.94 & 2 & Yes & \\
4 & 306.22 & 435.95 & 2 & Yes & \\
5 & 306.14 & 435.94 & 2 & Yes & \\
6 & 306.05 & 435.98 & 2 & Yes & \\
7 & 306.05 & 436.04 & 2 & Yes & \\
8 & 305.98 & 436.09 & 2 & Yes & \\
9 & 305.78 & 435.98 & 2 & Yes & \\
10 & 37.73 & 70.99 & 2 & Yes & B from P3 \\
11 & 31.8 & 39.85 & 2 & Yes & B from P3 \\
12 & 28.7 & 42.26 & 2 & Yes & B from P3 \\
13 & 28.05 & 42.27 & 2 & Yes & B from P3 \\
14 & 27.16 & 39.17 & 2 & Yes & B from P3 \\
15 & 23.6 & 41.5 & 2 & Yes & \\
16 & 23.07 & 38.93 & 2 & No & B from P3 \\
17 & 18.32 & 40.69 & 2 & No & B from P3 \\
18 & 17.19 & 35.05 & 2 & No & B from P3 \\
19 & 15.8 & 40.18 & 2 & No & B from P3 \\
20 & 15.64 & 44.26 & 2 & No & B from P3 \\
21 & 15.3 & 33.19 & 2 & No & B from P3 \\
22 & 15.19 & 27.91 & 2 & No & B from P3 \\
23 & 14.68 & 32.4 & 2 & No & B from P3 \\
24 & 14.38 & 49.11 & 2 & No & B from P3 \\
25 & 13.81 & 31.69 & 2 & No & B from P3 \\
26 & 12.96 & 28.72 & 2 & No & B from P3 \\
27 & 12.73 & 41.19 & 2 & No & B from P3 \\
28 & 12.38 & 38.56 & 2 & No & B from P3 \\
29 & 11.91 & 39.29 & 2 & No & B from expt \\
30 & 9.77 & 59.06 & 2 & No & B from expt \\
31 & 9.55 & 29.18 & 2 & No & B from expt \\
32 & 9.37 & 30.03 & 2 & No & B from expt \\
\hline & & & & & \\
\hline
\end{tabular}

Table A49. Molecular orbital data for pyrrole. Double-ionization threshold $=23.93 \mathrm{eV}$ from CCSD(T)/cc-pV(T+d)Z (to triplet dication).

\begin{tabular}{llllll}
\hline MO & B/eV & U/eV & N & DblIon & Remarks \\
\hline 1 & 424.51 & 601.93 & 2 & Yes & \\
2 & 306.12 & 436.02 & 2 & Yes & \\
3 & 306.12 & 436.03 & 2 & Yes & \\
4 & 304.95 & 435.82 & 2 & Yes & \\
5 & 304.92 & 436.13 & 2 & Yes & \\
6 & 35.12 & 48.63 & 2 & Yes & \\
7 & 27.81 & 44.2 & 2 & Yes & B from P3 \\
8 & 26.61 & 43.75 & 2 & Yes & \\
9 & 21.59 & 39.46 & 2 & No & \\
10 & 20.88 & 39.08 & 2 & No & \\
11 & 20.04 & 30.84 & 2 & No & \\
12 & 14.71 & 38.1 & 2 & No & B from P3 \\
13 & 14.5 & 37.92 & 2 & No & B from P3 \\
14 & 13.42 & 31.97 & 2 & No & B from P3 \\
15 & 13.57 & 36.46 & 2 & No & B from P3 \\
16 & 13.08 & 36.94 & 2 & No & B from P3 \\
17 & 9.2 & 34.46 & 2 & No & B from expt \\
18 & 8.209 & 30.04 & 2 & No & B from expt \\
\hline
\end{tabular}


Table A50. Molecular orbital data for pyridine. Double-ionization threshold $=25.53 \mathrm{eV}$ from CCSD(T)/cc-pV(T+d)Z (to triplet dication).

\begin{tabular}{llllll}
\hline MO & $\mathbf{B} / \mathbf{e V}$ & $\mathbf{U} / \mathbf{e V}$ & $\mathbf{N}$ & DblIon & Remarks \\
\hline 1 & 423.46 & 602.12 & 2 & Yes & \\
2 & 306.81 & 436.05 & 2 & Yes & \\
3 & 306.81 & 436.05 & 2 & Yes & \\
4 & 306.42 & 435.98 & 2 & Yes & \\
5 & 305.94 & 435.96 & 2 & Yes & \\
6 & 305.94 & 435.99 & 2 & Yes & \\
7 & 34.29 & 48.54 & 2 & Yes & \\
8 & 29.83 & 44.8 & 2 & Yes & B from P3 \\
9 & 28.19 & 43.43 & 2 & Yes & B from P3 \\
10 & 20.43 & 41.7 & 2 & No & B from P3 \\
11 & 23.35 & 41.83 & 2 & No & B from P3 \\
12 & 17.55 & 27.02 & 2 & No & B from P3 \\
13 & 16.12 & 42.38 & 2 & No & B from P3 \\
14 & 16.03 & 34.39 & 2 & No & B from P3 \\
15 & 14.74 & 32.85 & 2 & No & B from P3 \\
16 & 14.02 & 37.15 & 2 & No & B from P3 \\
17 & 13.3 & 27.41 & 2 & No & B from P3 \\
18 & 12.61 & 38.65 & 2 & No & B from expt \\
19 & 9.75 & 49.56 & 2 & No & B from expt \\
20 & 10.51 & 32.07 & 2 & No & B from expt \\
21 & 9.6 & 28.57 & 2 & No & B from expt \\
\hline
\end{tabular}

Table A51. Molecular orbital data for benzonitrile. Double-ionization threshold $=25.29 \mathrm{eV}$ from CCSD(T)/cc-pV(T+d)Z (to triplet dication).

\begin{tabular}{llllll}
\hline MO & B/eV & U/eV & N & DblIon & Remarks \\
\hline 1 & 423.77 & 602.28 & 2 & Yes & \\
2 & 307.28 & 435.95 & 2 & Yes & \\
3 & 306.91 & 435.83 & 2 & Yes & \\
4 & 306.76 & 435.98 & 2 & Yes & \\
5 & 306.76 & 436.01 & 2 & Yes & \\
6 & 306.62 & 435.93 & 2 & Yes & \\
7 & 306.47 & 436.0 & 2 & Yes & \\
8 & 306.47 & 436.08 & 2 & Yes & \\
9 & 33.38 & 56.44 & 2 & Yes & B from P3 \\
10 & 32.22 & 42.82 & 2 & Yes & \\
11 & 28.97 & 43.58 & 2 & Yes & \\
12 & 28.56 & 42.34 & 2 & Yes & \\
13 & 24.22 & 42.1 & 2 & No & \\
14 & 23.42 & 38.66 & 2 & No & B from P3 \\
15 & 18.61 & 33.24 & 2 & No & B from P3 \\
16 & 17.05 & 35.26 & 2 & No & B from P3 \\
17 & 16.1 & 38.88 & 2 & No & B from P3 \\
18 & 15.6 & 35.09 & 2 & No & B from P3 \\
19 & 15.19 & 32.32 & 2 & No & B from P3 \\
20 & 13.33 & 50.61 & 2 & No & B from P3 \\
21 & 13.71 & 25.63 & 2 & No & B from P3 \\
22 & 13.07 & 42.33 & 2 & No & B from P3 \\
23 & 13.17 & 37.05 & 2 & No & B from P3 \\
24 & 12.09 & 32.52 & 2 & No & B from expt \\
25 & 11.84 & 38.55 & 2 & No & B from expt \\
26 & 10.17 & 28.22 & 2 & No & B from expt \\
27 & 9.71 & 31.62 & 2 & No & B from expt \\
\hline & & & & & \\
\hline
\end{tabular}




\section{Supplemental Materials}

- $\quad$ Text files, named “<molecule $>$.bun,” containing molecular orbital data needed for computing TICS (51 files). These are the same data shown in the Appendix, but in computer-readable form.

- Perl script "beb_tbl.pl” that takes a BUN file as input for computing TICS.

- Text files, named “<molecule>_estPICS.txt," containing the PICS results in computer-readable form (50 files). These are the same data shown in Tables 2 to 51, but in computer-readable form.

\section{Acknowledgment}

I thank Dr. Jennifer Eigenbrode (NASA) for guidance on the molecules of interest, and for bringing this topic to my attention. 


\section{References}

[1] Mahaffy PR, Webster CR, Cabane M, Conrad PG, Coll P, Atreya SK, Arvey R, Barciniak M, Benna M, Bleacher L, Brinckerhoff WB, Eigenbrode JL, Carignan D, Cascia M, Chalmers RA, Dworkin JP, Errigo T, Everson P, Franz H, Farley R, Feng S, Frazier G, Freissinet C, Glavin DP, Harpold DN, Hawk D, Holmes V, Johnson CS, Jones A, Jordan P, Kellogg J, Lewis J, Lyness E, Malespin CA, Martin DK, Maurer J, McAdam AC, McLennan D, Nolan TJ, Noriega M, Pavlov AA, Prats B, Raaen E, Sheinman O, Sheppard D, Smith J, Stern JC, Tan F, Trainer M, Ming DW, Morris RV, Jones J, Gundersen C, Steele A, Wray J, Botta O, Leshin LA, Owen T, Battel S, Jakosky BM, Manning H, Squyres S, Navarro-Gonzalez R, McKay CP, Raulin F, Sternberg R, Buch A, Sorensen P, Kline-Schoder R, Coscia D, Szopa C, Teinturier S, Baffes C, Feldman J, Flesch G, Forouhar S, Garcia R, Keymeulen D, Woodward S, Block BP, Arnett K, Miller R, Edmonson C, Gorevan S, Mumm E (2012) The Sample Analysis at Mars Investigation and Instrument Suite. Space Science Reviews 170(1-4):401-478. https://doi.org/10.1007/s11214012-9879-z

[2] Nasser E (1971) Fundamentals of Gaseous Ionization and Plasma Electronics (Wiley-Interscience, New York).

[3] Tian CC, Vidal CR (1998) Electron impact dissociative ionization of $\mathrm{CO}_{2}$ : Measurements with a focusing time-of-flight mass spectrometer. Journal of Chemical Physics 108(3):927-936. https://doi.org/10.1063/1.475456

[4] Irikura KK (2016) Semi-empirical estimation of ion-specific cross sections in electron ionization of molecules. Journal of Chemical Physics 145(22):224102. https://doi.org/10.1063/1.4971242

[5] Kim Y-K, Rudd ME (1994) Binary-encounter-dipole model for electron-impact ionization. Physical Review A 50(5):3954-3967. https://link.aps.org/doi/10.1103/PhysRevA.50.3954

[6] Hwang W, Kim Y-K, Rudd ME (1996) New model for electron-impact ionization cross sections of molecules. Journal of Chemical Physics 104(8):2956-2966. https://doi.org/10.1063/1.471116

[7] Kim Y-K, Rudd ME (1999) Theory for ionization of molecules by electrons. Comments on Atomic and Molecular Physics 34(36):309-320.

[8] Huo WM, Kim Y-K (2000) Use of relativistic effective core potentials in the calculation of total electron-impact ionization cross-sections. Chemical Physics Letters 319(5-6):576-586. https://doi.org/10.1016/S0009-2614(00)00150-0

[9] Kim Y-K, Irikura KK, Ali MA (2000) Electron-impact total ionization cross sections of molecular ions. Journal of Research of the National Institute of Standards and Technology 105(2):285-291. https://doi.org/10.6028/jres.105.032

[10] Kim Y-K, Santos JP, Parente F (2000) Extension of the binary-encounter-dipole model to relativistic incident electrons. Physical Review A 62(5):052710. https://link.aps.org/doi/10.1103/PhysRevA.62.052710

[11] Kim Y-K, Irikura KK, Rudd ME, Zucker DS, Zucker MA, Coursey JS, Olsen KJ, Wiersma GG (2001) Electron-Impact Ionization Cross Section Database. Available at http://physics.nist.gov/ionxsec.

[12] Scott GE, Irikura KK (2005) Electron-impact ionization cross-sections of molecules containing heavy elements $(Z>10)$. Journal of Chemical Theory and Computation 1:1153-1161. https://doi.org/10.1021/ct050077j

[13] Scott GE, Irikura KK (2005) Performance of binary-encounter Bethe (BEB) theory for electron-impact ionization cross-sections of molecules containing heavy elements $(Z>10)$. Surface and Interface Analsis 37:973-977. https://doi.org/10.1002/sia.2091

[14] Linstrom PJ, Mallard WG (2016) NIST Chemistry WebBook. NIST Standard Reference Database Number 69 (National Institute of Standards and Technology, Gaithersburg, MD). Available at http://webbook.nist.gov/chemistry/

[15] Di Palma TM, Apicella B, Armenante M, Velotta R, Wang X, Spinelli N (2004) Dissociative electron impact ionization of methyl tert-butyl ether: Total ionization cross-section and kinetic energy distributions. Chemica Physics Letters 400(1-3):191195. https://doi.org/10.1016/j.cplatt.2004.10.106

[16] Feil S, Bacher A, Zangerl M, Schustereder W, Gluch K, Scheier P (2004) Discrimination effects for ions with initial kinetic energy produced by electron ionization of $\mathrm{C}_{2} \mathrm{H}_{2}$ in a Nier-type ion source. International Jorunal of Mass Spectrometry 233(13):325-333. https://doi.org/10.1016/j.ijms.2004.01.013

[17] Gluch G, Feil S, Scheier P, Schustereder W, Tepnual T, Feketeova L, Mair C, Matt-Leubner S, Stamatovic A, Märk TD (2005) Partial and differential electron impact ionization cross-sections for small hydrocarbon molecules. Nuclear Fusion Research: Understanding Plasma-Surface Interactions, Springer Series in Chemical Physics, Vol. 78, eds Clark REH, Reiter DH (Springer, Heidelberg, Germany), pp 437-456. https://doi.org/10.1007/3-540-27362-X_20

[18] Christophorou LG, Olthoff JK (2004) Fundamental Electron Interactions with Plasma Processing Gases (Kluwer Academic/Plenum, New York), p 776.

[19] Tian CC, Vidal CR (1998) Cross sections of the electron impact dissociative ionization of CO, $\mathrm{CH}_{4}$ and $\mathrm{C}_{2} \mathrm{H}_{2}$. Journal of Physics B-Atomic, Molecular, and Optical Physics 31(4):895-909. https://doi.org/10.1088/0953-4075/31/4/031

[20] Pandey A, Bapat B (2014) Effect of transmission losses on measured parameters in multi-ion coincidence momentum spectrometers. International Journal of Mass Spectrometry 361:23-27. https://doi.org/10.1016/j.ijms.2014.01.022

[21] Lee C, Yang W, Parr RG (1988) Development of the Colle-Salvetti correlation-energy formula into a functional of the electron density. Physical Review B 37:785-789. https://doi.org/10.1103/PhysRevB.37.785

[22] Becke AD (1993) Density-functional thermochemistry. III. The role of exact exchange. J Chemical Physics 98(7):5648-5652. https://doi.org/10.1063/1.464913

[23] Bergner A, Dolg M, Küchle W, Stoll H, Preuß H (1993) Ab initio energy-adjusted pseudopotentials for elements of groups 1317. Molecular Physics 80(6):1431-1441. https://doi.org/10.1080/00268979300103121

[24] Ortiz JV (1996) Partial third-order quasiparticle theory: Comparisons for closed-shell ionization energies and an application to the Borazine photoelectron spectrum. Journal of Chemical Physics 104(19):7599-7605. https://doi.org/10.1063/1.471468

[25] Raghavachari K, Trucks GW, Pople JA, Head-Gordon M (1989) A fifth-order perturbation comparison of electron correlation theories. Chemical Physics Letters 157(6):479-483. https://doi.org/10.1016/S0009-2614(89)87395-6

[26] Wilson AK, Dunning TH, Jr. (2003) $\mathrm{SO}_{2}$ revisited: Impact of tight $d$ augmented correlation consistent basis sets on structure and energetics. Journal of Chemical Physics 119(22):11712-11714. https://doi.org/10.1063/1.1624591 
[27] Frisch MJ, Trucks GW, Schlegel HB, Scuseria GE, Robb MA, Cheeseman JR, Scalmani G, Barone V, Mennucci B, Petersson GA, Nakatsuji H, Caricato M, Hratchian HP, Izmaylov AF, Bloino J, Zheng G, Sonnenberg JL, Hada M, Ehara M, Toyota K, Fukuda R, Hasegawa J, Ishida M, Nakajima T, Honda Y, Kitao O, Nakai H, Vreven T, Montgomery JA, Jr., Peralta JE, Ogliaro F, Bearpark M, Heyd JJ, Brothers E, Kudin KN, Staroverov VN, Kobayashi R, Normand J, Raghavachari K, Rendell A, Burant JC, Iyengar SS, Tomasi J, Cossi M, Rega N, Millam JM, Klene M, Knox JE, Cross JB, Bakken V, Adamo C, Jaramillo J, Gomperts R, Stratmann RE, Yazyev O, Austin AJ, Cammi R, Pomelli C, Ochterski JW, Martin RL, Morokuma K, Zakrzewski VG, Voth GA, Salvador P, Dannenberg JJ, Dapprich S, Daniels AD, Farkas O, Foresman JB, Ortiz JV, Cioslowski J, Fox DJ (2013) Gaussian 09 (Gaussian, Inc., Wallingford, CT), vers. D.01. Available at http://gaussian.com/glossary/g09/

[28] Kimura K, Katsumata S, Achiba Y, Yamazaki T, Iwata S (1981) Handbook of HeI Photoelectron Spectra of Fundamental Organic Molecules (Japan Scientific Societies, Tokyo).

[29] Howell JO, Goncalves JM, Amatore C, Klasinc L, Wightman RM, Kochi JK (1984) Electron transfer from aromatic hydrocarbons and their p-complexes with metals. Comparison of the standard oxidation potentials and vertical ionization potentials. Journal of the American Chemical Society 106(14):3968-3976. https://doi.org/10.1021/ja00326a014

[30] Kobayashi T, Arai T, Sakuragi H, Tokumaru K, Utsunomiya C (1981) A new method for conformational analysis by photoelectron spectroscopy with application to alkyl-substituted styrenes. Bulletin of the Chemical Society of Japan 54(6):16581661. https://doi.org/10.1246/bcsj.54.1658

[31] Krause DA, Taylor JW, Fenske RF (1978) An analysis of the effects of alkyl substituents on the ionization potentials of nalkenes. Journal of the American Chemical Society 100(3):718-723. https://doi.org/10.1021/ja00471a009

[32] Mintz DM, Kuppermann A (1979) Photoelectron spectroscopy of ethylene, isobutylene, trimethylethylene, and tetramethylethylene at variable angle. Journal of Chemical Physsics 71(8):3499-3513. https://doi.org/10.1063/1.438739

[33] Ashmore FS, Burgess AR (1978) Photoelectron spectra of the unbranched $\mathrm{C}_{5}-\mathrm{C}_{7}$ alkenes, aldehydes and ketones. Journal of the Chemical Society, Faraday Transactions 2-Molecular and Chemical Physics 74(0):734-742. https://doi.org/10.1039/f29787400734

[34] Baker AD, May DP, Turner DW (1968) Molecular photoelectron spectroscopy. 7. Vertical ionisation potentials of benzene and some of its monosubstituted and 1,4-disubstituted derivatives. Journal of the Chemical Society B-Physical Organic (1):22-34. https://doi.org/10.1039/j29680000022

[35] Kobayashi T, Nagakura S (1974) Photoelectron spectra of substituted benzenes. Bulletin of the Chemical Society of Japan 47(10):2563-2572. https://doi.org/10.1246/bcsj.47.2563

[36] Derrick PJ, Åsbrink L, Edqvist O, Jonsson B-Ö, Lindholm E (1971) Rydberg series in small molecules: XII. Photoelectron spectroscopy and electronic structure of pyrrole. International Journal of Mass Spectrometry and Ion Physics 6(3):191-202. https://doi.org/10.1016/0020-7381(71)80003-7

[37] Ausloos P, Clifton CL, Lias SG, Mikaya AI, Stein SE, Tchekhovskoi DV, Sparkman OD, Zaikin V, Zhu D (1999) The critical evaluation of a comprehensive mass spectral library. Journal of the American Society for Mass Spectrometry 10(4):287-299. https://doi.org/10.1016/S1044-0305(98)00159-7

[38] Anonymous ed (2014) NIST/EPA/NIH Mass Spectral Library (National Institute of Standards and Technology, Gaithersburg, MD). Available at http://chemdata.nist.gov/

About the author: Karl Irikura is a research chemist in the Chemical Sciences Division at NIST. The National Institute of Standards and Technology is an agency of the U.S. Department of Commerce. 